\title{
Controlling Asymmetric Remote and Cascade 1,3-Dipolar Cycloaddition Reactions by Organocatalysis
}

Pernille H. Poulsen, Stefania Vergura, Alicia Monleón, Danny Kaare Bech Jørgensen, and Karl Anker Jørgensen*

Department of Chemistry, Aarhus University

DK-8000 Aarhus C, Denmark

e-mail: kaj@chem.au.dk

\section{Contents}

1. General methods $\quad S 2$

2. Synthesis of aldehydes $\mathbf{1 c}$ and $\mathbf{1 d} \quad$ S3

3. Synthesis of nitrones $\mathbf{2 a - n , p , q}$ S5

4. The asymmetric remote 1,3-dipolar cycloaddition of nitrones to 2,4-dienals $\quad$ S10

4.1. General procedure for the organocatalytic synthesis of 4a-j $\quad$ S10

$\begin{array}{lr}\text { 4.2. Results and characterization } & \mathrm{S} 10\end{array}$

5. The asymmetric 1,3-dipolar cycloaddition cascade of nitrones to 2,4-dienals $\quad$ S15

5.1. General procedure for the organocatalytic synthesis of 5a-j S15

5.2. Results and characterization $\quad \mathrm{S} 15$

6. Functionalization of isoxazolidine-substituted enal 4a S22

6.1. General procedure for the organocatalytic synthesis of $6 a, b \quad S 22$

6.2. Results and characterization $\quad \mathrm{S} 22$

6.3. Organocatalytic synthesis of $7 \quad S 23$

7. The asymmetric triple 1,3-dipolar cycloaddition cascade of nitrones to 2,4,6- S24 trienals

7.1. General procedure for the organocatalytic synthesis of $\mathbf{8 a , b} \quad$ S24

$\begin{array}{lr}\text { 7.2. Results and characterization } & \text { S24 }\end{array}$

$\begin{array}{lr}\text { 8. Reductive ring-opening of } 5 c & \text { S27 }\end{array}$

9. Determination of the absolute configuration $\quad \mathrm{S} 28$

$\begin{array}{ll}\text { 10. References } & \text { S29 }\end{array}$

11. NMR Spectra $\quad$ S30

12. $\mathrm{UPC}^{2}$ traces $\quad \mathrm{S} 69$ 


\section{General methods}

NMR spectra were acquired on a Bruker AVANCE III HD spectrometer running at $400 \mathrm{MHz}$ for ${ }^{1} \mathrm{H}$ and 100 $\mathrm{MHz}$ for ${ }^{13} \mathrm{C}$. Chemical shifts $(\delta)$ are reported in ppm relative to residual solvent signals $\left(\mathrm{CHCl}_{3}, 7.26 \mathrm{ppm}\right.$ for ${ }^{1} \mathrm{H} \mathrm{NMR}, \mathrm{CDCl}_{3}, 77.0 \mathrm{ppm}$ for $\left.{ }^{13} \mathrm{C} \mathrm{NMR}\right)$. The following abbreviations are used to indicate the multiplicity in NMR spectra: $s$, singlet; $d$, doublet; $t$, triplet; $q$, quartet; $p$, pentet; $m$, multiplet; bs, broad signal; dd, double doublet; ddd, double double doublet; $\mathrm{dt}$, double triplet; $\mathrm{td}$, triple doublet; $\mathrm{dq}$, double quartet. ${ }^{13} \mathrm{C}$ spectra were acquired in broad band decoupled mode. For characterization of isomeric mixtures * denotes minor isomer, " denotes overlap of signals of both isomers, whereas no sign denotes signal of major isomer. The number of protons given in parenthesis is the sum over both isomers. Mass spectra were recorded on a Bruker Maxis Impact mass spectrometer using electronspray $\left(\mathrm{ES}^{+}\right)$ionization (referenced to the mass of the charged species). $\mathrm{CHCl}_{3}$ was dried over $3 \AA \mathrm{MS}$, THF was distilled from Na-benzophenone, other dry solvents were obtained from a MBraun MB SPS-800 solvent purification system. Analytical thin layer chromatography (TLC) was performed using pre-coated aluminium-backed plates (Merck Kieselgel 60 F254) and visualized by UV radiation, $\mathrm{KMnO}_{4}$ or $p$-anisaldehyde stains. For flash chromatography (FC) silica gel (Silica gel 60, 230- 400 mesh, Sigma-Aldrich) or latrobeads (latrobeads 6RS-8060, Mitsubishi Chemical Medience Corporation) were used. Optical rotations were measured on a Bellingham+Stanley ADP440+ polarimeter, $\alpha$ values are given in $\mathrm{deg} \cdot \mathrm{cm}^{3} \cdot \mathrm{g}^{-1} \cdot \mathrm{dm}^{-1}$; concentration $\mathrm{c}$ in $\mathrm{g} \cdot(100 \mathrm{ml})^{-1}$. The enantiomeric excess (ee) of the products was determined by Ultraperformance Convergence Chromatography (Waters ACQUITY UPC $^{2}$ ) using Daicel Chiralpak IA, IB, IC, ID, and AMY columns as chiral stationary phases. Racemates for UPC ${ }^{2}$ analysis of compounds 4 were made applying a 1:1 mixture of catalyst $3 \mathrm{c}$ and ent-3c in the reaction. Racemates for UPC ${ }^{2}$ analysis of compounds $\mathbf{5 , 6}$, and $\mathbf{8}$ were prepared using a mixture of products obtained from reactions with catalysts $3 \mathrm{c}$ and ent-3c, respectively. Racemate for UPC ${ }^{2}$ analysis of compound $\mathbf{7}$ was

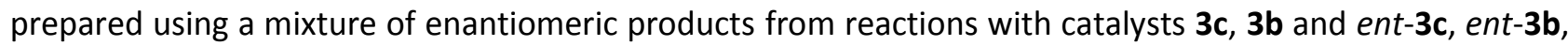
respectively. Catalyst $\mathbf{3 c}$ was synthesized according to the literature. ${ }^{1}$ Unless otherwise noted, analytical grade solvents and commercially available reagents were used without further purification. 


\section{Synthesis of aldehydes $1 \mathrm{c}$ and $1 \mathrm{~d}$}

(2E,4E)-7-Phenylhepta-2,4-dienal, 1c

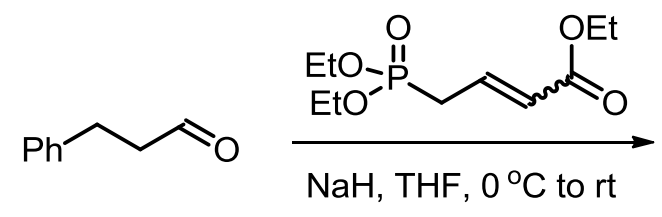

$\overbrace{1 \mathrm{Ph}}^{\mathrm{Ph}}$

Triethyl 4-phosphonocrotonate ( $1.95 \mathrm{~mL}, 8.8 \mathrm{mmol}, 1.1$ equiv) was added dropwise to a stirred suspension of $\mathrm{NaH}$ (352 mg, $8.8 \mathrm{mmol}, 1.1$ equiv, $60 \%$ oil dispersion) in dry THF (16 mL) at $0{ }^{\circ} \mathrm{C}$ under Ar atmosphere. The mixture was stirred for $1 \mathrm{~h}$ before a solution of hydrocinnamaldehyde $(1.05 \mathrm{~mL}, 8.0 \mathrm{mmol}, 1.0$ equiv) in THF $(8 \mathrm{~mL})$ was added dropwise. The reaction was allowed to warm to room temperature and stirred until completion, monitored by TLC. The reaction was quenched with sat. aq. $\mathrm{NH}_{4} \mathrm{Cl}(10 \mathrm{~mL})$ and the aqueous phase was extracted with $\mathrm{Et}_{2} \mathrm{O}(3 \times 10 \mathrm{~mL})$. The combined organic phase was washed with water $(20 \mathrm{~mL})$, brine $(3 \times 20 \mathrm{~mL})$, and dried over $\mathrm{MgSO}_{4}$. The solvent was removed. The crude product was used in the next step without further purification.

DIBAL (24.0 mL, $24.0 \mathrm{mmol}, 3.0$ equiv, $1.0 \mathrm{M}$ in toluene) was added dropwise to a stirred solution of the diene ester in dry toluene $(40 \mathrm{~mL})$ under Ar atmosphere at $-78{ }^{\circ} \mathrm{C}$. The reaction was stirred for $15 \mathrm{~min}$ before it was quenched by carefully adding water $(8 \mathrm{~mL})$. The mixture was allowed to warm to room temperature before sat. aq. Rochelle salt $(50 \mathrm{~mL})$ was added. The mixture was stirred overnight. The aqueous phase was extracted with $\mathrm{CH}_{2} \mathrm{Cl}_{2}(3 \times 30 \mathrm{~mL})$ and the combined organic phase dried over $\mathrm{MgSO}_{4}$. The solvent was removed. The crude product was used in the next step without further purification.

The alcohol was dissolved in dry $\mathrm{CH}_{2} \mathrm{Cl}_{2}(200 \mathrm{~mL})$ and activated $\mathrm{MnO}_{2}(7.65 \mathrm{~g}, 88.0$ mmol, 11.0 equiv) was added. After stirring vigorously for $1.5 \mathrm{~h}$ the solid was filtered off and washed with EtOAc. The solvent was removed. The aldehyde $1 \mathrm{c}$ was isolated by FC on silica (EtOAc/pentane 2:98 to 8:92) in 41\% yield over 3 steps (a mixture of isomers was obtained, but a fraction of the major isomer could be isolated) as a pale yellow oil.

${ }^{1} \mathrm{H} \mathrm{NMR}\left(400 \mathrm{MHz}, \mathrm{CDCl}_{3}\right) \delta 9.53(\mathrm{~d}, J=8.0 \mathrm{~Hz}, 1 \mathrm{H}), 7.33-7.27(\mathrm{~m}, 2 \mathrm{H}), 7.25-7.15(\mathrm{~m}, 3 \mathrm{H}), 7.07(\mathrm{dd}, J=15.3$, $9.7 \mathrm{~Hz}, 1 \mathrm{H}), 6.44-6.20(\mathrm{~m}, 2 \mathrm{H}), 6.07(\mathrm{dd}, J=15.3,8.0 \mathrm{~Hz}, 1 \mathrm{H}), 2.79(\mathrm{t}, J=7.7 \mathrm{~Hz}, 2 \mathrm{H}), 2.55(\mathrm{dt}, J=9.2,6.5 \mathrm{~Hz}$, $2 \mathrm{H})$.

${ }^{13} \mathrm{C} \mathrm{NMR}\left(100 \mathrm{MHz}, \mathrm{CDCl}_{3}\right) \delta 193.9,152.5,145.7,140.8,130.4,129.2,128.5$ (2C), 128.4 (2C), 126.2, 34.9, 34.8 .

HRMS calculated for: $\left[\mathrm{C}_{13} \mathrm{H}_{14} \mathrm{O}+\mathrm{H}\right]^{+}$187.1117; found: 187.1118 . 

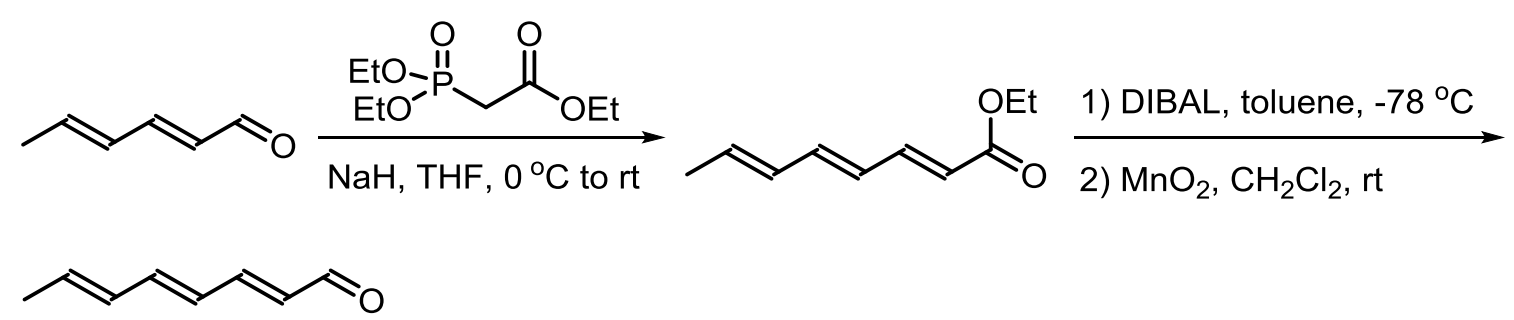

1d

Following the procedure above employing triethyl phosphonoacetate and hexa-2,4-dienal, the aldehyde 1d was isolated by FC on silica (EtOAc/pentane $5: 95$ ) in $57 \%$ yield over 3 steps ( $E / E: E / Z 14: 1$ ) as a pale yellow solid.

${ }^{1} \mathrm{H} \mathrm{NMR}\left(400 \mathrm{MHz}, \mathrm{CDCl}_{3}\right) \delta 9.57 *(\mathrm{~d}, J=8.2 \mathrm{~Hz}, 1 \mathrm{H}), 9.55(\mathrm{~d}, J=8.0 \mathrm{~Hz}, 1 \mathrm{H}), 7.23-7.15^{*}(\mathrm{~m}, 1 \mathrm{H}), 7.11(\mathrm{dd}, J=$ 15.2, $11.2 \mathrm{~Hz}, 1 \mathrm{H}), 7.04-6.94 *(\mathrm{~m}, 1 \mathrm{H}), 6.64(\mathrm{dd}, J=14.9,10.7 \mathrm{~Hz}, 1 \mathrm{H}), 6.43^{*}(\mathrm{dd}, J=14.9,11.2 \mathrm{~Hz}, 1 \mathrm{H}), 6.34$ (dd, $J=15.0,11.1 \mathrm{~Hz}, 1 \mathrm{H}), 6.25-5.99^{\#}(\mathrm{~m}, 5 \mathrm{H}), 5.87^{*}(\mathrm{dd}, J=10.7,7.3 \mathrm{~Hz}, 1 \mathrm{H}), 1.86^{\#}(\mathrm{~d}, J=6.9 \mathrm{~Hz}, 6 \mathrm{H})$.

${ }^{13} \mathrm{C} \mathrm{NMR}\left(100 \mathrm{MHz}, \mathrm{CDCl}_{3}\right) \delta 193.6^{\#}(2 \mathrm{C}), 152.5,152.3^{*}, 143.1,137.5^{*}, 137.1,133.5^{*}, 131.1,131.0^{*}, 130.6$, $129.5^{*}, 128.7^{*}, 127.6,18.7,13.9 *$.

HRMS calculated for: $\left[\mathrm{C}_{8} \mathrm{H}_{10} \mathrm{O}+\mathrm{H}\right]^{+}$123.0804; found: 123.0805 . 


\section{Synthesis of nitrones $2 a-n, p, q$}

\section{Procedure 1:}

To a solution of $N$-benzylhydroxylamine hydrochloride $(1.60 \mathrm{~g}, 10.0 \mathrm{mmol}, 1.0$ equiv) in MeOH (15 mL) $6 \mathrm{M}$ $\mathrm{NaOH}$ was added dropwise until $\mathrm{pH} \sim 9$. The reaction mixture was concentrated in vacuo before $\mathrm{CH}_{2} \mathrm{Cl}_{2}(10$ $\mathrm{mL}$ ) and brine $(10 \mathrm{~mL})$ were added. The phases were separated and the aqueous phase extracted with $\mathrm{CH}_{2} \mathrm{Cl}_{2}(2 \times 10 \mathrm{~mL})$. The combined organic phase was dried over $\mathrm{MgSO}_{4}$ and concentrated in vacuo. The crude $\mathrm{N}$-benzylhydroxylamine, aldehyde (10.0 mmol, 1.0 equiv), and $\mathrm{MgSO}_{4}(1.20 \mathrm{~g}, 10.0 \mathrm{mmol}, 1.0$ equiv) were stirred in $\mathrm{Et}_{2} \mathrm{O}(40 \mathrm{~mL})$ overnight at room temperature. The reaction mixture was filtered washing with $\mathrm{CH}_{2} \mathrm{Cl}_{2}$ and concentrated in vacuo.

\section{Procedure 2: ${ }^{2}$}

$\mathrm{N}$-Phenylhydroxylamine ( $327 \mathrm{mg}, 3.0 \mathrm{mmol}, 1.0$ equiv) and aldehyde ( $3.0 \mathrm{mmol}, 1.0$ equiv) were dissolved in $\mathrm{CH}_{2} \mathrm{Cl}_{2}\left(10 \mathrm{~mL}\right.$ ) before $\mathrm{CaCl}_{2}$ (166 mg, $1.5 \mathrm{mmol}, 0.5$ equiv) was added. The reaction mixture was stirred at room temperature until completion. Then, it was filtered and the solvent removed.

\section{Procedure $3:^{3}$}

Aldehyde (2.0 mmol, 1.0 equiv), $\mathrm{N}$-methylhydroxylamine hydrochloride (334 $\mathrm{mg}, 4.0 \mathrm{mmol}, 2.0$ equiv), $\mathrm{Na}_{2} \mathrm{CO}_{3}$ (477 mg, $4.5 \mathrm{mmol}, 2.2$ equiv), and $\mathrm{Na}_{2} \mathrm{SO}_{4}(142 \mathrm{mg}, 1.0 \mathrm{mmol}, 0.5$ equiv) were added to a mortar and ground until completion. $\mathrm{Et}_{2} \mathrm{O}(2 \mathrm{~mL})$ was added, the mixture filtered, and concentrated in vacuo.

\section{(Z)-N-(2-Methylbenzylidene)-1-phenylmethanamine oxide, 2a}

Be

Following procedure 1 the nitrone $\mathbf{2 a}$ was isolated by FC on silica (EtOAc/pentane 33:67 to $50: 50)$ in $37 \%$ yield as a colorless solid.

${ }^{1} \mathrm{H} \mathrm{NMR}\left(400 \mathrm{MHz}, \mathrm{CDCl}_{3}\right)$ 8 9.17-9.11 (m, $\left.1 \mathrm{H}\right), 7.54(\mathrm{~s}, 1 \mathrm{H})$, 7.53-7.46 (m, 2H), 7.46-7.37 (m, $3 \mathrm{H}), 7.28-7.26(\mathrm{~m}, 2 \mathrm{H}), 7.19-7.14(\mathrm{~m}, 1 \mathrm{H}), 5.09(\mathrm{~s}, 2 \mathrm{H}), 2.28(\mathrm{~s}, 3 \mathrm{H})$.

${ }^{13} \mathrm{C}$ NMR $\left(100 \mathrm{MHz}, \mathrm{CDCl}_{3}\right) \delta 136.2,133.3,131.2,130.2,130.1,129.2$ (2C), 129.0 (3C), 128.7, 127.8, 126.3, 71.7, 19.7.

HRMS calculated for: $\left[\mathrm{C}_{15} \mathrm{H}_{15} \mathrm{NO}+\mathrm{H}\right]^{+} 226.1226$; found: 226.1226 .

(Z)-N-(2-Bromobenzylidene)-1-phenylmethanamine oxide, 2b<smiles>[O-]/[N+](=C/c1ccccc1Br)c1ccccc1</smiles>

Following procedure 1 the nitrone $\mathbf{2} \mathbf{b}$ was isolated by FC on silica (EtOAc/pentane 20:80 to $33: 67)$ in $71 \%$ yield as a colorless solid. 
${ }^{1} \mathrm{H} \mathrm{NMR}\left(400 \mathrm{MHz}, \mathrm{CDCl}_{3}\right) \delta 9.28(\mathrm{~d}, J=8.0 \mathrm{~Hz}, 1 \mathrm{H}), 7.90(\mathrm{~s}, 1 \mathrm{H}), 7.59(\mathrm{~d}, J=8.0 \mathrm{~Hz}, 1 \mathrm{H}), 7.53-7.47(\mathrm{~m}, 2 \mathrm{H})$, 7.46-7.39 (m, 3H), $7.36(\mathrm{t}, J=7.7 \mathrm{~Hz}, 1 \mathrm{H}), 7.22(\mathrm{t}, J=7.7 \mathrm{~Hz}, 1 \mathrm{H}), 5.10(\mathrm{~s}, 2 \mathrm{H})$.

${ }^{13} \mathrm{C} \mathrm{NMR}\left(100 \mathrm{MHz}, \mathrm{CDCl}_{3}\right) \delta 132.9,132.8,132.7,131.3,129.4,129.4,129.3$ (2C), 129.1, 129.0 (2C), 127.6, 123.4, 72.0.

HRMS calculated for: $\left[\mathrm{C}_{14} \mathrm{H}_{12} \mathrm{BrNO}+\mathrm{H}\right]^{+} 290.0175,292.0155$; found: $290.0179,292.0160$.

\section{(Z)-N-(Naphthalen-1-ylmethylene)-1-phenylmethanamine oxide, 2c}<smiles>[O-][N+]([O-])=Cc1cccc2ccccc12</smiles>

Following procedure 1 the nitrone $2 \mathrm{c}$ was isolated by FC on silica (EtOAc/pentane 50:50) in $85 \%$ yield as a colorless solid.

Analytical data were in accordance with the literature. ${ }^{4}$

\section{(Z)-N-((4-Methoxynaphthalen-1-yl)methylene)-1-phenylmethanamine oxide, 2d}<smiles>COc1ccc(/C=[N+](/[O-])Cc2ccccc2)c2ccccc12</smiles>

Following procedure 1 the nitrone $\mathbf{2} \mathbf{d}$ was isolated by $\mathrm{FC}$ on silica $\left(\mathrm{CH}_{2} \mathrm{Cl}_{2} / \mathrm{EtOAC}\right.$ $100: 0$ to $80: 20$ ) in $63 \%$ yield as a colorless solid.

${ }^{1} \mathrm{H}$ NMR $\left(400 \mathrm{MHz}, \mathrm{CDCl}_{3}\right) \delta 9.66(\mathrm{~d}, J=8.5 \mathrm{~Hz}, 1 \mathrm{H}), 8.33(\mathrm{dd}, J=8.2,1.3 \mathrm{~Hz}, 1 \mathrm{H}), 8.09$ $(\mathrm{s}, 1 \mathrm{H}), 7.82(\mathrm{~d}, J=8.0 \mathrm{~Hz}, 1 \mathrm{H}), 7.56-7.39(\mathrm{~m}, 7 \mathrm{H}), 6.88(\mathrm{~d}, J=8.5 \mathrm{~Hz}, 1 \mathrm{H}), 5.17(\mathrm{~s}, 2 \mathrm{H})$, $4.04(\mathrm{~s}, 3 \mathrm{H})$.

${ }^{13} \mathrm{C} \mathrm{NMR}\left(100 \mathrm{MHz}, \mathrm{CDCl}_{3}\right) \delta 157.2,133.6,131.9,129.9,129.2(2 \mathrm{C}), 129.0$ (2C), 128.9, 128.4, 127.4, 125.5, $125.2,123.0,121.1,118.2,103.6,71.6,55.7$.

HRMS calculated for: $\left[\mathrm{C}_{19} \mathrm{H}_{17} \mathrm{NO}_{2}+\mathrm{H}\right]^{+}$292.1332; found: 292.1336 .

(Z)-N-((1-Bromonaphthalen-2-yl)methylene)-1-phenylmethanamine oxide, 2e<smiles>[O-][N+](=Cc1ccc2ccccc2c1Br)c1ccccc1</smiles>

Following procedure 1 the nitrone $\mathbf{2 e}$ was isolated by FC on silica (EtOAc/pentane $20: 80$ ) in $14 \%$ yield as a colorless solid.

${ }^{1} \mathrm{H} N M R\left(400 \mathrm{MHz}, \mathrm{CDCl}_{3}\right) \delta 9.14(\mathrm{~d}, J=8.8 \mathrm{~Hz}, 1 \mathrm{H}), 8.33-8.31(\mathrm{~m}, 1 \mathrm{H}), 8.13(\mathrm{~s}, 1 \mathrm{H})$, 7.84-7.80 (m, 2H), 7.61-7.53 (m, 4H), 7.48-7.40 (m, 3H), $5.16(\mathrm{~s}, 2 \mathrm{H})$.

${ }^{13} \mathrm{C} \mathrm{NMR}\left(100 \mathrm{MHz}, \mathrm{CDCl}_{3}\right) \delta 135.1,134.0,133.0,132.0,129.4$ (2C), 129.2, 129.1 (2C), 128.2, 128.2, 128.0, 127.7 (2C), 127.6, 125.3, 124.7, 72.2.

HRMS calculated for: $\left[\mathrm{C}_{18} \mathrm{H}_{14} \mathrm{BrNO}+\mathrm{H}\right]^{+} 340.0332,342.0311$; found: $340.0331,342.0307$. 
(Z)-N-(2-Methylbenzylidene)methanamine oxide, $2 f$

$\Theta \quad$ Following procedure 3 (reaction time $5 \mathrm{~min}$ ) the nitrone $\mathbf{2 f}$ was isolated by FC on silica (EtOAc/pentane 10:90) in $80 \%$ yield as a colorless solid.

(1)

${ }^{1} \mathrm{H}$ NMR $\left(400 \mathrm{MHz}, \mathrm{CDCl}_{3}\right)$ 8 9.14-9.10 (m, 1H), $7.53(\mathrm{~s}, 1 \mathrm{H}), 7.32-7.28(\mathrm{~m}, 2 \mathrm{H}), 7.22-7.18(\mathrm{~m}$, $1 \mathrm{H}), 3.92(\mathrm{~s}, 3 \mathrm{H}), 2.39(\mathrm{~s}, 3 \mathrm{H})$.

${ }^{13} \mathrm{C}$ NMR $\left(100 \mathrm{MHz}, \mathrm{CDCl}_{3}\right) \delta 136.0,132.1,130.2,130.2,128.8,127.8,126.3,55.0,19.8$.

HRMS calculated for: $\left[\mathrm{C}_{9} \mathrm{H}_{11} \mathrm{NO}+\mathrm{H}\right]^{+}$150.0913; found: 150.0915.

\section{(Z)-N-(2-Methylbenzylidene)aniline oxide, $2 \mathrm{~g}$}<smiles>Cc1ccccc1/C=[N+](\[O-])c1ccccc1</smiles>

Following procedure 2 (reaction time $2 \mathrm{~h}$ ) the nitrone $\mathbf{2 g}$ was isolated by FC on silica (EtOAc/pentane/ $\mathrm{CH}_{2} \mathrm{Cl}_{2}$ 25:63:12) in $26 \%$ yield as a pale yellow solid.

${ }^{1} \mathrm{H} \mathrm{NMR}\left(400 \mathrm{MHz}, \mathrm{CDCl}_{3}\right)$ 8 9.42-9.33 (m, $\left.1 \mathrm{H}\right), 8.07(\mathrm{~s}, 1 \mathrm{H}), 7.82-7.73(\mathrm{~m}, 2 \mathrm{H}), 7.54-7.45(\mathrm{~m}$, $3 \mathrm{H}), 7.40-7.33(\mathrm{~m}, 2 \mathrm{H}), 7.28-7.23(\mathrm{~m}, 1 \mathrm{H}), 2.46(\mathrm{~s}, 3 \mathrm{H})$.

${ }^{13} \mathrm{C} \mathrm{NMR}\left(100 \mathrm{MHz}, \mathrm{CDCl}_{3}\right) \delta 149.8,136.9,131.8,130.7,130.3,129.9,129.2$ (2C), 129.0, 128.0, 126.5, 122.0 (2C), 20.0 .

HRMS calculated for: $\left[\mathrm{C}_{14} \mathrm{H}_{13} \mathrm{NO}+\mathrm{H}\right]^{+}$212.1070; found: 212.1069 .

\section{(Z)-N-Benzylidene-1-phenylmethanamine oxide, $2 \mathrm{~h}$}

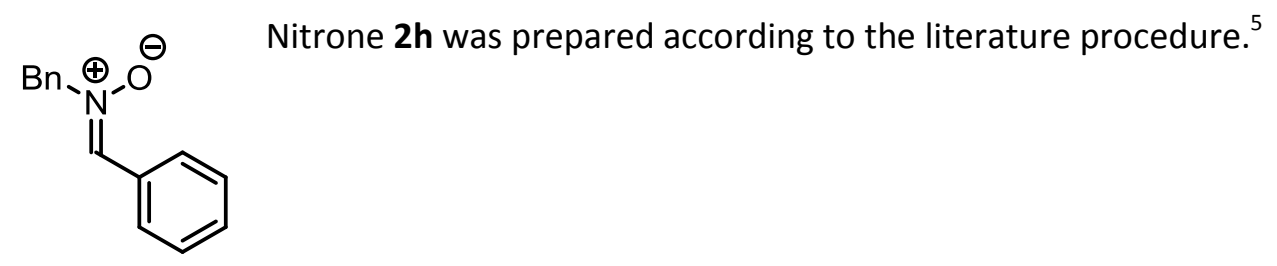

(Z)-N-(4-Methoxybenzylidene)-1-phenylmethanamine oxide, 2i<smiles>COc1ccc(/C=[N+](\[O-])Cc2ccccc2)cc1</smiles>

Following procedure 1 the nitrone $\mathbf{2} \mathbf{i}$ was isolated by FC on silica (EtOAc/pentane $33: 67$ to $67: 33$ ) in $47 \%$ yield as a colorless solid.

Analytical data were in accordance with the literature. ${ }^{6}$ 
(Z)-N-(4-Bromobenzylidene)-1-phenylmethanamine oxide, $2 \mathrm{j}$<smiles>[O-][N+](=Cc1ccc(Br)cc1)c1ccccc1</smiles>

Following procedure 1 the nitrone $\mathbf{2} \mathbf{j}$ was isolated by FC on silica (EtOAc/pentane 25:75 to $50: 50$ ) in $52 \%$ yield as a colorless solid.

Analytical data were in accordance with the literature. ${ }^{6}$

(Z)-N-(2-Methoxybenzylidene)-1-phenylmethanamine oxide, 2k<smiles>COc1ccccc1/C=[N+](\[O-])Cc1ccccc1</smiles>

Following procedure 1 the nitrone $\mathbf{2 k}$ was isolated by FC on silica (EtOAc/pentane/ $\mathrm{CH}_{2} \mathrm{Cl}_{2}$ $42: 42: 16)$ in $79 \%$ yield as a colorless solid.

Analytical data were in accordance with the literature. ${ }^{7}$

(Z)-N-(3-Methylbenzylidene)-1-phenylmethanamine oxide, 2I<smiles>Cc1cccc(/C=[N+](\[O-])Cc2ccccc2)c1</smiles>

Following procedure 1 the nitrone $2 \mathbf{l}$ was isolated by $\mathrm{FC}$ on silica $\left(\mathrm{CH}_{2} \mathrm{Cl}_{2} /\right.$ EtOAc $\left.95: 5\right)$ in $51 \%$ yield as a colorless solid.

${ }^{1} \mathrm{H}$ NMR $\left(400 \mathrm{MHz}, \mathrm{CDCl}_{3}\right) \delta 8.14(\mathrm{~s}, 1 \mathrm{H}), 7.92(\mathrm{~d}, J=7.8 \mathrm{~Hz}, 1 \mathrm{H}), 7.49-7.46(\mathrm{~m}, 2 \mathrm{H})$, 7.43-7.37 (m, 4H), $7.29(\mathrm{t}, J=7.7 \mathrm{~Hz}, 1 \mathrm{H}), 7.22(\mathrm{~d}, J=7.6 \mathrm{~Hz}, 1 \mathrm{H}), 5.05(\mathrm{~s}, 2 \mathrm{H}), 2.36(\mathrm{~s}$, $3 \mathrm{H})$.

${ }^{13} \mathrm{C}$ NMR $\left(100 \mathrm{MHz}, \mathrm{CDCl}_{3}\right) \delta 138.1,134.4,133.2,131.3,130.3,129.2$ (2C), 129.0, 128.9 (3C), 128.3, 125.9, 71.2, 21.4 .

HRMS calculated for: $\left[\mathrm{C}_{15} \mathrm{H}_{15} \mathrm{NO}+\mathrm{H}\right]^{+}$226.1226; found: 226.1227 .

\section{(Z)-N-(Naphthalen-2-ylmethylene)-1-phenylmethanamine oxide, $2 \mathrm{~m}$}<smiles>[O-]/[N+](=C\c1ccc2ccccc2c1)c1ccccc1</smiles>

Following procedure 1 the nitrone $\mathbf{2 m}$ was isolated by FC on silica (EtOAc/pentane $50: 50$ ) in $61 \%$ yield as a colorless solid.

Analytical data were in accordance with the literature. ${ }^{8}$ 


\section{(Z)-N-Benzylidenemethanamine oxide, $2 n$}

$\Theta \quad$ Following procedure 3 (reaction time $5 \mathrm{~min}$ ) the nitrone $\mathbf{2 n}$ was isolated by $\mathrm{FC}$ on silica (EtOAc/pentane $50: 50$ ) in $50 \%$ yield as a colorless solid.

II

Analytical data were in accordance with the literature. ${ }^{3}$

(Z)-N-(4-Methoxybenzylidene)aniline oxide, $2 p$<smiles>COc1ccc(/C=[N+](\[O-])c2ccccc2)cc1</smiles>

Following procedure 2 (reaction time $1 \mathrm{~h}$ ) the nitrone $2 p$ was obtained directly in quantitative yield without further purification as a pale brown solid.

Analytical data were in accordance with the literature. ${ }^{9}$

(Z)-N-(Naphthalen-2-ylmethylene)aniline oxide, 2q<smiles>[O-][N+](=Cc1ccc2ccccc2c1)c1ccccc1</smiles>

Following procedure 2 (reaction time $4 \mathrm{~h}$ ) the nitrone $\mathbf{2 q}$ was isolated by FC on silica (EtOAc/pentane/ $\mathrm{CH}_{2} \mathrm{Cl}_{2} 25: 63: 12$ ) in $37 \%$ yield as a pale brown solid.

Analytical data were in accordance with the literature. ${ }^{10}$ 


\section{The asymmetric remote 1,3-dipolar cycloaddition of nitrones to 2,4-dienals}

\subsection{General procedure for the organocatalytic synthesis of $4 a-j$}

A glass vial equipped with a magnetic stirring bar was charged with catalyst $3 \mathrm{c}(15.7 \mathrm{mg}, 0.02 \mathrm{mmol}, 0.2$ equiv), nitrone 2 ( $0.1 \mathrm{mmol}, 1.0$ equiv), and dry $\mathrm{CHCl}_{3}(0.45 \mathrm{~mL})$. Aq. sat. $\mathrm{KCl}(5.4 \mu \mathrm{L}, 0.025 \mathrm{mmol}, 0.25$ equiv) was added. The mixture was cooled to $4{ }^{\circ} \mathrm{C}$ before addition of aldehyde $1(0.2 \mathrm{mmol}, 2.0$ equiv) and a solution of $\mathrm{TfOH}$ in $\mathrm{CHCl}_{3}(50 \mu \mathrm{L}, 0.005 \mathrm{mmol}, 0.05$ equiv, $0.1 \mathrm{M})$. The reaction mixture was stirred at $4{ }^{\circ} \mathrm{C}$ until completion. The diastereomeric ratio was determined by ${ }^{1} \mathrm{H}$ NMR analysis of the crude reaction mixture and the isolated product by comparing the integrals of the peaks corresponding to the double bond protons of the diastereoisomeric products.

\subsection{Results and characterization}

(E)-3-((3S,4R,5S)-2-Benzyl-5-methyl-3-(o-tolyl)isoxazolidin-4-yl)acrylaldehyde, 4a<smiles>COc1ccccc1[C@@H]1[C@H](/C=C/C=O)[C@H](C)ON1Cc1ccccc1</smiles>

Following the general procedure (reaction time $5 \mathrm{~d}$ ) the product 4a was isolated by FC on silica (EtOAc/pentane 5:95 to 15:85) in $80 \%$ yield as a yellow oil.

${ }^{1} \mathrm{H} \mathrm{NMR}\left(400 \mathrm{MHz}, \mathrm{CDCl}_{3}\right) \delta 9.52(\mathrm{~d}, J=7.7 \mathrm{~Hz}, 1 \mathrm{H}), 7.65(\mathrm{dd}, J=7.7,1.0 \mathrm{~Hz}, 1 \mathrm{H}), 7.33-$ $7.20(\mathrm{~m}, 6 \mathrm{H}), 7.17(\mathrm{td}, J=7.4,1.4 \mathrm{~Hz}, 1 \mathrm{H}), 7.10(\mathrm{~d}, J=7.3 \mathrm{~Hz}, 1 \mathrm{H}), 6.83$ (dd, $J=15.6,9.3$ $\mathrm{Hz}, 1 \mathrm{H}), 5.99(\mathrm{dd}, J=15.6,7.7 \mathrm{~Hz}, 1 \mathrm{H}), 4.26(\mathrm{p}, J=6.2 \mathrm{~Hz}, 1 \mathrm{H}), 4.12(\mathrm{~d}, J=8.6 \mathrm{~Hz}, 1 \mathrm{H})$, $4.01(\mathrm{~d}, J=14.2 \mathrm{~Hz}, 1 \mathrm{H}), 3.85(\mathrm{~d}, J=14.2 \mathrm{~Hz}, 1 \mathrm{H}), 3.08(\mathrm{~m}, 1 \mathrm{H}), 2.24(\mathrm{~s}, 3 \mathrm{H}), 1.42(\mathrm{~d}, J=6.1 \mathrm{~Hz}, 3 \mathrm{H})$.

${ }^{13} \mathrm{C} \mathrm{NMR}\left(100 \mathrm{MHz}, \mathrm{CDCl}_{3}\right) \delta 193.0,153.8,137.3,135.9,135.7,134.1,130.6,128.5$ (2C), 128.2 (2C), 127.7, $127.5,127.1,126.7,78.0,72.4,63.7,59.9,19.9,19.8$.

HRMS calculated for: $\left[\mathrm{C}_{21} \mathrm{H}_{23} \mathrm{NO}_{2}+\mathrm{H}\right]^{+}$322.1802; found: 322.1806. The ee was determined by UPC ${ }^{2}$ using a Chiralpak IA-3 column [1\% iPrOH (0.5 min), then gradient from $1 \%$ to $40 \%(10 \% / \mathrm{min}), 120 \mathrm{bar}, 40{ }^{\circ} \mathrm{C}$; flow rate $3.0 \mathrm{~mL} / \mathrm{min} ; \tau_{\text {major }}=2.67 \mathrm{~min}, \tau_{\text {minor }}=2.99 \mathrm{~min}(93 \% \mathrm{ee}) .[\alpha]_{\mathrm{D}}^{25}=-66.6\left(\mathrm{c}=1.0, \mathrm{CH}_{2} \mathrm{Cl}_{2}\right)$.

(E)-3-((3S,4R,5S)-2-Benzyl-3-(2-bromophenyl)-5-methylisoxazolidin-4-yl)acrylaldehyde, 4b

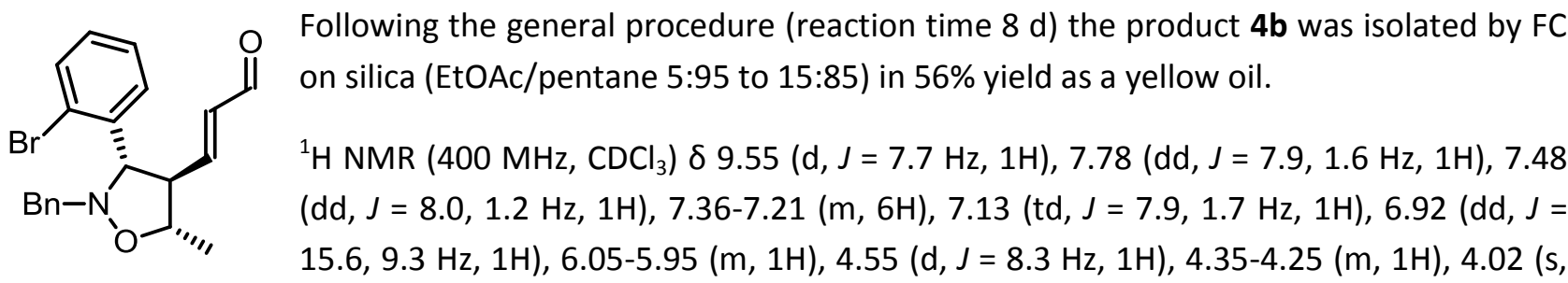
2H), $2.99(\mathrm{dd}, J=16.1,8.8 \mathrm{~Hz}, 1 \mathrm{H}), 1.37(\mathrm{~d}, J=6.1 \mathrm{~Hz}, 3 \mathrm{H})$.

${ }^{13} \mathrm{C} \mathrm{NMR}\left(100 \mathrm{MHz}, \mathrm{CDCl}_{3}\right) \delta 193.2,153.2,137.6,136.8,134.1,132.8,129.3,129.3,128.7$ (2C), $128.2(2 \mathrm{C})$, $128.1,127.3,123.7,77.9,73.9,64.3,60.6,19.1$. 
HRMS calculated for: $\left[\mathrm{C}_{20} \mathrm{H}_{20} \mathrm{BrNO}_{2}+\mathrm{H}\right]^{+} 386.0751,38.0730$; found: $386.0756,388.0737$. The ee was determined by UPC $^{2}$ using a Chiralpak IC-3 column [ $1 \%$ iPrOH $(0.5 \mathrm{~min})$, then gradient from $1 \%$ to $40 \%$ $\left.(10 \% / \mathrm{min}), 120 \mathrm{bar}, 40{ }^{\circ} \mathrm{C}\right]$; flow rate $3.0 \mathrm{~mL} / \mathrm{min} ; \tau_{\text {major }}=3.86 \mathrm{~min}, \tau_{\text {minor }}=3.75 \mathrm{~min}(81 \% \mathrm{ee}) .[\alpha]_{\mathrm{D}}^{25}=-35.4$ (c $=0.8, \mathrm{CH}_{2} \mathrm{Cl}_{2}$ ).

\section{(E)-3-((3S,4R,5S)-2-Benzyl-5-methyl-3-(naphthalen-1-yl)isoxazolidin-4-yl)acrylaldehyde, 4c}

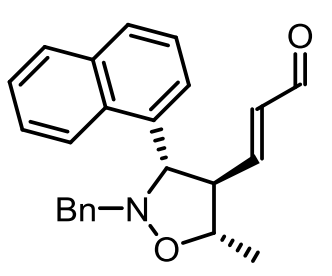

Following the general procedure (reaction time $24 \mathrm{~h}$ ) the product $\mathbf{4 c}$ was isolated by FC on silica (EtOAc/pentane 5:95 to 10:90) in 73\% yield as a colorless oil.

${ }^{1} \mathrm{H}$ NMR $\left(400 \mathrm{MHz}, \mathrm{CDCl}_{3}\right) \delta 9.49(\mathrm{~d}, J=7.7 \mathrm{~Hz}, 1 \mathrm{H}), 8.28-8.19(\mathrm{~m}, 1 \mathrm{H})$, 7.91-7.84 $(\mathrm{m}$, $1 \mathrm{H}), 7.81(\mathrm{~d}, J=8.2 \mathrm{~Hz}, 1 \mathrm{H}), 7.75(\mathrm{~d}, J=7.1 \mathrm{~Hz}, 1 \mathrm{H}), 7.56-7.40(\mathrm{~m}, 3 \mathrm{H}), 7.37-7.14(\mathrm{~m}$, $5 \mathrm{H}), 6.88(\mathrm{dd}, J=15.6,9.1 \mathrm{~Hz}, 1 \mathrm{H}), 5.90(\mathrm{dd}, J=15.6,7.7 \mathrm{~Hz}, 1 \mathrm{H}), 4.56(\mathrm{~d}, J=8.5 \mathrm{~Hz}$, $1 \mathrm{H}), 4.36(\mathrm{p}, J=6.2 \mathrm{~Hz}, 1 \mathrm{H}), 4.07(\mathrm{~d}, J=14.3 \mathrm{~Hz}, 1 \mathrm{H}), 3.95(\mathrm{~d}, J=14.2 \mathrm{~Hz}, 1 \mathrm{H}), 3.31(\mathrm{q}, J=8.1 \mathrm{~Hz}, 1 \mathrm{H}), 1.46$ (d, $J=6.1 \mathrm{~Hz}, 3 \mathrm{H}$ ).

${ }^{13} \mathrm{C} \mathrm{NMR}\left(100 \mathrm{MHz}, \mathrm{CDCl}_{3}\right) \delta 192.9,153.8,137.2,134.3,134.1,133.4,131.2,129.0,128.8,128.6$ (2C), 128.2 (2C), 127.1, 126.1, 125.8, 125.7, 125.5, 123.5, 78.1, 73.7, 62.7, 60.4, 19.8 .

HRMS calculated for: $\left[\mathrm{C}_{24} \mathrm{H}_{23} \mathrm{NO}_{2}+\mathrm{H}\right]^{+} 358.1802$; found: 358.1802 . The ee was determined by $\mathrm{UPC}^{2}$ using a Chiralpak ID-3 column [1\% iPrOH (0.5 min), then gradient from $1 \%$ to $40 \%(10 \% / \mathrm{min}), 120$ bar, $40{ }^{\circ} \mathrm{C}$; f flow rate $3.0 \mathrm{~mL} / \mathrm{min} ; \tau_{\text {major }}=3.18 \mathrm{~min}, \tau_{\text {minor }}=3.29 \mathrm{~min}\left(92 \%\right.$ ee). $[\alpha]^{25}=-27.8\left(c=1.0, \mathrm{CH}_{2} \mathrm{Cl}_{2}\right)$.

\section{(E)-3-((3S,4R,5S)-2-Benzyl-3-(4-methoxynaphthalen-1-yl)-5-methylisoxazolidin-4-yl)acrylaldehyde, 4d}<smiles>COc1ccc([C@@H]2/C(=C/C=O)[C@H](C)ON2Cc2ccccc2)c2ccccc12</smiles>

Following the general procedure (reaction time $48 \mathrm{~h}$ ) the product $\mathbf{4 d}$ was isolated by FC on silica (EtOAc/pentane 5:95 to 15:85) in $85 \%$ yield as a colorless oil.

${ }^{1} \mathrm{H}$ NMR $\left(400 \mathrm{MHz}, \mathrm{CDCl}_{3}\right) \delta 9.48(\mathrm{~d}, J=7.7 \mathrm{~Hz}, 1 \mathrm{H}), 8.34-8.32(\mathrm{~m}, 1 \mathrm{H}), 8.26(\mathrm{~d}, J=8.2$ $\mathrm{Hz}, 1 \mathrm{H}), 7.62(\mathrm{~d}, J=8.0 \mathrm{~Hz}, 1 \mathrm{H}), 7.56-7.48(\mathrm{~m}, 2 \mathrm{H}), 7.32-7.25(\mathrm{~m}, 4 \mathrm{H}), 7.24-7.19(\mathrm{~m}$, $1 \mathrm{H}), 6.85$ (dd, $J=15.6,9.0 \mathrm{~Hz}, 1 \mathrm{H}), 6.80(\mathrm{~d}, J=8.1 \mathrm{~Hz}, 1 \mathrm{H}), 5.91(\mathrm{dd}, J=15.6,7.7 \mathrm{~Hz}$, $1 \mathrm{H}), 4.45(\mathrm{~d}, J=8.1 \mathrm{~Hz}, 1 \mathrm{H}), 4.34(\mathrm{p}, J=6.2 \mathrm{~Hz}, 1 \mathrm{H}), 4.06(\mathrm{~d}, J=14.3 \mathrm{~Hz}, 1 \mathrm{H}), 4.01(\mathrm{~s}$, $3 \mathrm{H}), 3.89(\mathrm{~d}, J=14.3 \mathrm{~Hz}, 1 \mathrm{H}), 3.33(\mathrm{dd}, J=15.7,8.6 \mathrm{~Hz}, 1 \mathrm{H}), 1.48(\mathrm{~d}, J=5.9 \mathrm{~Hz}, 3 \mathrm{H})$.

${ }^{13} \mathrm{C}$ NMR $\left(100 \mathrm{MHz}, \mathrm{CDCl}_{3}\right) \delta 193.0,155.6,154.1,137.4,134.2,132.1,128.6$ (2C), 128.1(2C), 127.1, 126.6, $126.3,126.0,125.1,123.3,122.8,103.4,78.0,74.0,62.2,60.2,55.5,19.9$.

HRMS calculated for: $\left[\mathrm{C}_{25} \mathrm{H}_{25} \mathrm{NO}_{3}+\mathrm{H}\right]^{+} 388.1907$; found: 388.1910 . The ee was determined by $\mathrm{UPC}^{2}$ using a Chiralpak ID-3 column [1\% iPrOH (0.5 min), then gradient from $1 \%$ to $40 \%(10 \% / \mathrm{min}), 120 \mathrm{bar}, 40{ }^{\circ} \mathrm{C}$; flow rate $3.0 \mathrm{~mL} / \mathrm{min} ; \tau_{\text {major }}=3.50 \mathrm{~min}, \tau_{\text {minor }}=3.66 \mathrm{~min}\left(94 \%\right.$ ee). $[\alpha]_{D}^{25}=-56.4\left(\mathrm{c}=1.0, \mathrm{CH}_{2} \mathrm{Cl}_{2}\right)$. 


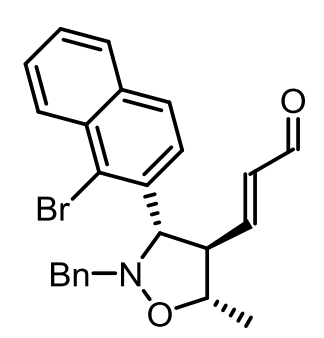

Following the general procedure (reaction time $7 \mathrm{~d}$ ) the product $4 \mathrm{e}$ was isolated by FC on silica (EtOAc/pentane 5:95 to 10:90) in 63\% yield as a yellow oil.

${ }^{1} \mathrm{H}$ NMR $\left(400 \mathrm{MHz}, \mathrm{CDCl}_{3}\right) \delta 9.54(\mathrm{~d}, J=7.7 \mathrm{~Hz}, 1 \mathrm{H}), 9.05^{*}(\mathrm{~d}, J=7.8 \mathrm{~Hz}, 1 \mathrm{H}), 8.30-8.24^{\#}$ $(\mathrm{m}, 2 \mathrm{H}), 7.89-7.79^{\#}(\mathrm{~m}, 6 \mathrm{H}), 7.55-7.45^{\#}(\mathrm{~m}, 4 \mathrm{H}), 7.43-7.39^{*}(\mathrm{~m}, 2 \mathrm{H})$, 7.37-7.31 $(\mathrm{m}, 2 \mathrm{H})$, 7.30-7.23 $(\mathrm{m}, 4 \mathrm{H}), 7.23-7.17^{\#}(\mathrm{~m}, 2 \mathrm{H}), 6.97^{\#}(\mathrm{dd}, J=15.6,9.2 \mathrm{~Hz}, 2 \mathrm{H}), 6.35^{*}(\mathrm{dd}, J=$ 15.5, $9.9 \mathrm{~Hz}, 1 \mathrm{H}$ ), 5.98 (ddd, $J=15.7,7.7,0.9 \mathrm{~Hz}, 1 \mathrm{H}$ ), $4.92(\mathrm{~d}, J=8.5 \mathrm{~Hz}, 1 \mathrm{H}), 4.80^{*}(\mathrm{~d}$, $J=9.3 \mathrm{~Hz}, 1 \mathrm{H}), 4.37(\mathrm{dq}, J=7.4,6.1 \mathrm{~Hz}, 1 \mathrm{H}), 4.19-4.11^{*}(\mathrm{~m}, 1 \mathrm{H}), 4.09-4.03^{\#}(\mathrm{~m}, 3 \mathrm{H}), 3.86^{*}(\mathrm{~d}, J=14.5 \mathrm{~Hz}$, $1 \mathrm{H}), 3.52-3.44^{*}(\mathrm{~m}, 1 \mathrm{H}), 3.13-3.04(\mathrm{~m}, 1 \mathrm{H}), 1.41(\mathrm{~d}, J=6.1 \mathrm{~Hz}, 3 \mathrm{H}), 1.38^{*}(\mathrm{~d}, J=6.1 \mathrm{~Hz}, 3 \mathrm{H})$.

${ }^{13} \mathrm{C}$ NMR $\left(100 \mathrm{MHz}, \mathrm{CDCl}_{3}\right) \delta 193.1,193.1^{*}, 153.7^{*}, 153.0,137.1^{*}, 136.9,135.6^{\#}(2 \mathrm{C}), 134.2^{\#}(3 \mathrm{C}), 134.1^{*}$, 132.3*, 132.0, $128.7(2 \mathrm{C}), 128.7^{*}(2 \mathrm{C}), 128.5^{*}(2 \mathrm{C}), 128.3^{*}(2 \mathrm{C}), 128.2(2 \mathrm{C}), 128.2^{*}, 128.1,128.0 *, 127.8$, $127.6,127.3^{*}, 127.3,127.2^{*}, 126.9^{\#}(2 \mathrm{C}), 125.7^{*}, 125.7,123.9^{\#}(2 \mathrm{C}), 78.2^{*}, 77.9,74.9,73.7^{*}, 64.1,60.7$, $60.3^{*}, 56.6^{*}, 19.0,18.0^{*}$.

HRMS calculated for: $\left[\mathrm{C}_{24} \mathrm{H}_{22} \mathrm{BrNO}_{2}+\mathrm{H}\right]^{+}$436.0907, 438.0886; found: 436.0906, 438.0893. The ee was determined by UPC ${ }^{2}$ using a Chiralpak IC-3 column [1\% iPrOH ( $0.5 \mathrm{~min}$ ), then gradient from $1 \%$ to $40 \%$ $\left.(10 \% / \mathrm{min}), 120 \mathrm{bar}, 40{ }^{\circ} \mathrm{C}\right]$; flow rate $3.0 \mathrm{~mL} / \mathrm{min} ; \tau_{\text {major }}=4.79 \mathrm{~min}, \tau_{\operatorname{minor}}=4.56 \mathrm{~min}(88 \% \mathrm{ee}) .[\alpha]_{\mathrm{D}}^{25}=-59.3$ (c $=1.0, \mathrm{CH}_{2} \mathrm{Cl}_{2}$ ).

\section{(E)-3-((3S,4R,5S)-2,5-Dimethyl-3-(o-tolyl)isoxazolidin-4-yl)acrylaldehyde, $4 \mathrm{f}$}<smiles>Cc1ccccc1[C@@H]1[C@H](/C=C/C=O)[C@H](C)ON1C</smiles>

Following the general procedure (reaction time $3 \mathrm{~d}$ ) the product $\mathbf{4} \mathbf{f}$ was isolated by FC on silica (EtOAc/pentane 5:95 to 15:85) in 66\% yield as a yellow oil.

${ }^{1} \mathrm{H}$ NMR $\left(400 \mathrm{MHz}, \mathrm{CDCl}_{3}\right) \delta 9.51(\mathrm{~d}, J=7.7 \mathrm{~Hz}, 1 \mathrm{H}), 7.53(\mathrm{~d}, J=7.4 \mathrm{~Hz}, 1 \mathrm{H}), 7.25-7.20$ $(\mathrm{m}, 1 \mathrm{H}), 7.18(\mathrm{td}, J=7.4,1.5 \mathrm{~Hz}, 1 \mathrm{H}), 7.14-7.11(\mathrm{~m}, 1 \mathrm{H}), 6.82(\mathrm{dd}, J=15.6,9.2 \mathrm{~Hz}, 1 \mathrm{H})$, $5.97(\mathrm{dd}, J=15.6,7.7 \mathrm{~Hz}, 1 \mathrm{H}), 4.28(\mathrm{p}, J=6.2 \mathrm{~Hz}, 1 \mathrm{H}), 3.93-3.86(\mathrm{~m}, 1 \mathrm{H}), 3.10(\mathrm{dd}, J=$ $15.6,8.8 \mathrm{~Hz}, 1 \mathrm{H}), 2.62(\mathrm{~s}, 3 \mathrm{H}), 2.30(\mathrm{~s}, 3 \mathrm{H}), 1.44(\mathrm{~d}, J=6.2 \mathrm{~Hz}, 3 \mathrm{H})$.

${ }^{13} \mathrm{C} \mathrm{NMR}\left(100 \mathrm{MHz}, \mathrm{CDCl}_{3}\right) \delta$ 193.0, 153.7, 136.1, 135.1, 134.1, 130.6, 127.8, 127.3, 126.7, 77.9, 75.0, 64.0, 43.6, 20.3, 19.9 .

HRMS calculated for: $\left[\mathrm{C}_{15} \mathrm{H}_{19} \mathrm{NO}_{2}+\mathrm{H}\right]^{+} 246.1489$; found: 246.1491 . The ee was determined by $\mathrm{UPC}^{2}$ using a Chiralpak IC-3 column [1\% iPrOH (0.5 min), then gradient from $1 \%$ to $40 \%(10 \% / \mathrm{min}), 120 \mathrm{bar}, 40{ }^{\circ} \mathrm{C}$; flow rate $3.0 \mathrm{~mL} / \mathrm{min} ; \tau_{\text {major }}=3.24 \mathrm{~min}, \tau_{\text {minor }}=2.93 \mathrm{~min}(93 \% \mathrm{ee}) .[\alpha]_{\mathrm{D}}^{25}=-72.0\left(\mathrm{c}=1.0, \mathrm{CH}_{2} \mathrm{Cl}_{2}\right)$.

(E)-3-((3S,4R,5S)-5-Methyl-2-phenyl-3-(o-tolyl)isoxazolidin-4-yl)acrylaldehyde, 4g<smiles>Cc1ccccc1[C@@H]1[C@H](/C=C/C=O)[C@H](C)ON1c1ccccc1</smiles>

Following the general procedure (reaction time $48 \mathrm{~h}$, temperature $-20^{\circ} \mathrm{C}$ ) the product $\mathbf{4 g}$ was isolated by FC on silica (EtOAc/pentane 5:95) in $99 \%$ yield as a yellow oil. 
${ }^{1} \mathrm{H} \mathrm{NMR}\left(400 \mathrm{MHz}, \mathrm{CDCl}_{3}\right) \delta 9.49(\mathrm{~d}, J=7.7 \mathrm{~Hz}, 1 \mathrm{H}), 7.72-7.67(\mathrm{~m}, 1 \mathrm{H})$, 7.30-7.14 $(\mathrm{m}, 5 \mathrm{H}), 6.97-6.91(\mathrm{~m}, 3 \mathrm{H})$, $6.70(\mathrm{dd}, J=15.6,9.7 \mathrm{~Hz}, 1 \mathrm{H}), 6.04(\mathrm{dd}, J=15.6,7.7 \mathrm{~Hz}, 1 \mathrm{H}), 4.88(\mathrm{~d}, J=7.1 \mathrm{~Hz}, 1 \mathrm{H}), 4.26(\mathrm{dq}, J=8.8,6.0 \mathrm{~Hz}$, $1 \mathrm{H}), 3.10(\mathrm{td}, J=9.2,7.4 \mathrm{~Hz}, 1 \mathrm{H}), 2.36(\mathrm{~s}, 3 \mathrm{H}), 1.42(\mathrm{~d}, J=6.0 \mathrm{~Hz}, 3 \mathrm{H})$.

${ }^{13} \mathrm{C} \mathrm{NMR}\left(100 \mathrm{MHz}, \mathrm{CDCl}_{3}\right) \delta 192.7,152.4,152.2,138.9,134.9,134.2,130.7,129.2(2 \mathrm{C}), 127.7,127.4,127.0$, $121.6,113.6(2 \mathrm{C}), 79.4,74.2,65.4,19.8,16.5$.

HRMS calculated for: $\left[\mathrm{C}_{20} \mathrm{H}_{21} \mathrm{NO}_{2}+\mathrm{H}\right]^{+} 308.1645$; found: 308.1646 . The ee was determined by $\mathrm{UPC}^{2}$ using a Chiralpak IC-3 column [1\% iPrOH (0.5 min), then gradient from $1 \%$ to $40 \%(10 \% / \mathrm{min}), 120$ bar, $40{ }^{\circ} \mathrm{C}$; flow rate $3.0 \mathrm{~mL} / \mathrm{min} ; \tau_{\text {major }}=3.64 \mathrm{~min}, \tau_{\text {minor }}=3.78 \mathrm{~min}(47 \% \mathrm{ee}) .[\alpha]_{\mathrm{D}}^{25}=-15.8\left(\mathrm{c}=1.0, \mathrm{CH}_{2} \mathrm{Cl}_{2}\right)$.

\section{(E)-3-((3S,4R,5S)-2-Benzyl-5-hexyl-3-(o-tolyl)isoxazolidin-4-yl)acrylaldehyde, $4 \mathrm{~h}$}

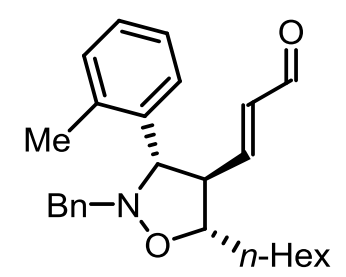

Following the general procedure (reaction time $7 \mathrm{~d}$ ) the product $\mathbf{4 h}$ was isolated by FC on silica (EtOAc/pentane 5:95 to 10:90) in 60\% yield as a yellow oil.

${ }^{1} \mathrm{H}$ NMR (400 MHz, CDCl $) \delta 9.52(\mathrm{~d}, J=7.7 \mathrm{~Hz}, 1 \mathrm{H}), 7.63(\mathrm{dd}, J=7.8,1.5 \mathrm{~Hz}, 1 \mathrm{H})$, 7.33-7.08 (m, 8H), $6.86(\mathrm{dd}, J=15.6,9.5 \mathrm{~Hz}, 1 \mathrm{H}), 5.97(\mathrm{dd}, J=15.6,7.7 \mathrm{~Hz}, 1 \mathrm{H}), 4.08$ $4.01(\mathrm{~m}, 2 \mathrm{H}), 3.98(\mathrm{~d}, J=14.4 \mathrm{~Hz}, 1 \mathrm{H}), 3.79(\mathrm{~d}, J=14.4 \mathrm{~Hz}, 1 \mathrm{H}), 3.12(\mathrm{td}, J=8.9,5.9$ $H z, 1 H), 2.24(s, 3 H), 1.96-1.82(m, 1 H), 1.67-1.58(m, 1 H), 1.42-1.18(m, 8 H), 0.93-0.83(m, 3 H)$.

${ }^{13} \mathrm{C} \mathrm{NMR}\left(100 \mathrm{MHz}, \mathrm{CDCl}_{3}\right) \delta 193.1,154.8,137.4,136.0,135.6,133.8,130.6,128.4(2 \mathrm{C}), 128.1(2 \mathrm{C}), 127.7$, $127.4,127.0,126.7,82.1,72.3,62.4,59.5,35.0,31.7,29.1,26.0,22.5,19.9,14.0$.

HRMS calculated for: $\left[\mathrm{C}_{26} \mathrm{H}_{33} \mathrm{NO}_{2}+\mathrm{H}\right]^{+}$392.2584; found: 392.2582. The ee was determined by $\mathrm{UPC}^{2}$ using a Chiralpak IA-3 column [1\% iPrOH (0.5 min), then gradient from $1 \%$ to $40 \%(10 \% / \mathrm{min}), 120 \mathrm{bar}, 40{ }^{\circ} \mathrm{C}$; flow rate $3.0 \mathrm{~mL} / \mathrm{min} ; \tau_{\text {major }}=2.75 \mathrm{~min}, \tau_{\text {minor }}=3.04 \mathrm{~min}(89 \% \mathrm{ee}) .[\alpha]_{\mathrm{D}}^{25}=-20.2\left(\mathrm{c}=1.0, \mathrm{CH}_{2} \mathrm{Cl}_{2}\right.$ ).

\section{(E)-3-((3S,4R,5S)-2-Benzyl-5-phenethyl-3-(o-tolyl)isoxazolidin-4-yl)acrylaldehyde, 4i}

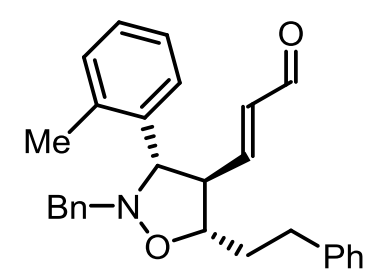

Following the general procedure (reaction time $4 \mathrm{~d}$ ) the product $4 \mathrm{i}$ was isolated by FC on silica (EtOAc/pentane 5:95 to $10: 90$ ) in $72 \%$ yield as a yellow oil.

${ }^{1} \mathrm{H}$ NMR $\left(400 \mathrm{MHz}, \mathrm{CDCl}_{3}\right) \delta 9.51(\mathrm{~d}, J=7.7 \mathrm{~Hz}, 1 \mathrm{H}), 7.64(\mathrm{dd}, J=7.6,1.6 \mathrm{~Hz}, 1 \mathrm{H})$, 7.44-7.03 (m, 13H), $6.83(\mathrm{dd}, J=15.6,9.5 \mathrm{~Hz}, 1 \mathrm{H}), 5.94(\mathrm{dd}, J=15.6,7.7 \mathrm{~Hz}, 1 \mathrm{H})$, 4.10-3.91 (m, 3H), $3.79(\mathrm{~d}, J=14.3 \mathrm{~Hz}, 1 \mathrm{H}), 3.15(\mathrm{~m}, 1 \mathrm{H}), 2.79-2.71(\mathrm{~m}, 1 \mathrm{H}), 2.67-$ $2.59(\mathrm{~m}, 1 \mathrm{H}), 2.30-2.16(\mathrm{~m}, 1 \mathrm{H}), 2.25(\mathrm{~s}, 3 \mathrm{H}), 1.96-1.87(\mathrm{~m}, 1 \mathrm{H})$.

${ }^{13} \mathrm{C}$ NMR $\left(100 \mathrm{MHz}, \mathrm{CDCl}_{3}\right) \delta 193.0,154.4,141.4,137.5,136.1,135.3,133.8,130.7,128.5$ (2C), $128.4(4 \mathrm{C})$, $128.2(2 \mathrm{C}), 127.8,127.4,127.1,126.8,126.0,81.1,72.4,62.4,59.4,36.8,32.3,19.9$.

HRMS calculated for: $\left[\mathrm{C}_{28} \mathrm{H}_{29} \mathrm{NO}_{2}+\mathrm{H}\right]^{+} 412.2271$; found: 412.2274 . The ee was determined by $\mathrm{UPC}^{2}$ using a Chiralpak IB-3 column [1\% iPrOH (0.5 min), then gradient from $1 \%$ to $40 \%(10 \% / \mathrm{min}), 120 \mathrm{bar}, 40{ }^{\circ} \mathrm{C}$; flow rate $3.0 \mathrm{~mL} / \mathrm{min} ; \tau_{\text {major }}=3.77 \mathrm{~min}, \tau_{\text {minor }}=4.05 \mathrm{~min}(90 \% \mathrm{ee}) .[\alpha]_{\mathrm{D}}^{25}=-17.2\left(\mathrm{c}=1.0, \mathrm{CH}_{2} \mathrm{Cl}_{2}\right)$. 
<smiles>COc1ccc([C@H]2[C@H](/C=C/C=O)ON(Cc3ccccc3)[C@@H]2CCc2ccccc2)c2ccccc12</smiles>

Following the general procedure (reaction time $24 \mathrm{~h}$ ) the product $\mathbf{4 j}$ was isolated by FC on silica (EtOAc/pentane 5:95 to 10:90) in 78\% yield as a yellow oil.

${ }^{1} \mathrm{H}$ NMR $\left(400 \mathrm{MHz}, \mathrm{CDCl}_{3}\right) \delta 9.49(\mathrm{~d}, J=7.7 \mathrm{~Hz}, 1 \mathrm{H}), 8.37-8.34(\mathrm{~m}, 1 \mathrm{H}), 8.25(\mathrm{~d}, J=$ $7.8 \mathrm{~Hz}, 1 \mathrm{H}), 7.66(\mathrm{~d}, J=7.9 \mathrm{~Hz}, 1 \mathrm{H}), 7.57-7.49(\mathrm{~m}, 2 \mathrm{H}), 7.38-7.18(\mathrm{~m}, 10 \mathrm{H}), 6.88$ (dd, $J=15.6,9.2 \mathrm{~Hz}, 1 \mathrm{H}), 6.82(\mathrm{~d}, J=8.1 \mathrm{~Hz}, 1 \mathrm{H}), 5.88(\mathrm{dd}, J=15.6 \mathrm{~Hz}, 7.7 \mathrm{~Hz}, 1 \mathrm{H})$, $4.43(\mathrm{~d}, J=6.6 \mathrm{~Hz}, 1 \mathrm{H}), 4.19-4.11(\mathrm{~m}, 1 \mathrm{H}), 4.07(\mathrm{~d}, J=14.3 \mathrm{~Hz}, 1 \mathrm{H}), 4.02(\mathrm{~s}, 3 \mathrm{H})$, $3.86(\mathrm{~d}, J=14.4 \mathrm{~Hz}, 1 \mathrm{H}), 3.42-3.36(\mathrm{~m}, 1 \mathrm{H}), 2.85-2.78(\mathrm{~m}, 1 \mathrm{H}), 2.73-2.65(\mathrm{~m}, 1 \mathrm{H}), 2.37-2.27(\mathrm{~m}, 1 \mathrm{H}), 2.04-$ $1.95(\mathrm{~m}, 1 \mathrm{H})$.

${ }^{13} \mathrm{C}$ NMR $\left(100 \mathrm{MHz}, \mathrm{CDCl}_{3}\right) \delta 193.0,155.6,154.7,141.4,137.6,134.0,132.2,128.5(2 \mathrm{C}), 128.5(2 \mathrm{C}), 128.4$ (2C), 128.1 (2C), 127.0, 126.7, 126.2, 126.0, 126.0, 125.1, 124.4, 123.2, 122.8, 103.5, 81.1, 73.8, 61.0, 59.8, $55.5,36.9,32.4$.

HRMS calculated for: $\left[\mathrm{C}_{32} \mathrm{H}_{31} \mathrm{NO}_{3}+\mathrm{H}\right]^{+} 478.2377$; found: 478.2382 . The ee was determined by $\mathrm{UPC}^{2}$ using a Chiralpak IA-3 column [1\% iPrOH (0.5 min), then gradient from $1 \%$ to $40 \%(10 \% / \mathrm{min}), 120 \mathrm{bar}, 40{ }^{\circ} \mathrm{C}$; flow rate $3.0 \mathrm{~mL} / \mathrm{min} ; \tau_{\text {major }}=4.04 \mathrm{~min}, \tau_{\text {minor }}=4.65 \mathrm{~min}(93 \% \mathrm{ee}) .[\alpha]_{\mathrm{D}}^{25}=-47.8\left(\mathrm{c}=1.0, \mathrm{CH}_{2} \mathrm{Cl}_{2}\right)$. 


\section{The asymmetric 1,3-dipolar cycloaddition cascade of nitrones to 2,4-dienals}

\subsection{General procedure for the organocatalytic synthesis of $5 a-j$}

A glass vial equipped with a magnetic stirring bar was charged with catalyst $3 \mathrm{c}(15.7 \mathrm{mg}, 0.02 \mathrm{mmol}, 0.2$ equiv), nitrone 2 ( $0.22 \mathrm{mmol}, 2.2$ equiv), and dry $\mathrm{CHCl}_{3}(0.45 \mathrm{~mL})$. Aq. sat. $\mathrm{KCl}(5.4 \mu \mathrm{L}, 0.025 \mathrm{mmol}, 0.25$ equiv) was added followed by addition of aldehyde 1 ( $0.1 \mathrm{mmol}, 1.0$ equiv). A solution of $\mathrm{TfOH}$ in $\mathrm{CHCl}_{3}$ (50 $\mu \mathrm{L}, 0.1 \mathrm{M}, 0.005 \mathrm{mmol}, 0.05$ equiv) was added and the reaction stirred at room temperature until completion. The diastereomeric ratio was determined by ${ }^{1} \mathrm{H}$ NMR analysis of the crude reaction mixture and the isolated product by comparing the integrals of the peaks corresponding to the aldehyde protons of the diastereoisomeric products.

\subsection{Results and characterization}

(3S,3'S,4R,4'R,5S,5'S)-2,2'-Dibenzyl-5-methyl-3,3'-diphenyl-[4,5'-biisoxazolidine]-4'-carbaldehyde, 5a<smiles>C[C@@H]1ON(Cc2ccccc2)[C@H](c2ccccc2)[C@H]1C[C@H]1ON(Cc2ccccc2)[C@H](c2ccccc2)[C@H]1C</smiles>

Following the general procedure (reaction time $24 \mathrm{~h}$ ) the product $\mathbf{5 a}$ was isolated by FC on silica (EtOAc/pentane 10:90 to $20: 80$ ) in $73 \%$ yield as a yellow oil.

${ }^{1} \mathrm{H} \mathrm{NMR}\left(400 \mathrm{MHz}, \mathrm{CDCl}_{3}\right) \delta 9.76(\mathrm{~d}, J=1.9 \mathrm{~Hz}, 1 \mathrm{H}), 7.46-7.10(\mathrm{~m}, 2 \mathrm{H}), 4.56(\mathrm{t}, J=$ $5.9 \mathrm{~Hz}, 1 \mathrm{H}), 4.41-4.30(\mathrm{~m}, 1 \mathrm{H}), 4.06(\mathrm{~d}, J=8.6 \mathrm{~Hz}, 1 \mathrm{H}), 3.91(\mathrm{dd}, J=14.3,3.1 \mathrm{~Hz}$, $2 \mathrm{H}), 3.72-3.58(\mathrm{~m}, 3 \mathrm{H}), 3.36(\mathrm{ddd}, J=8.3,5.8,1.9 \mathrm{~Hz}, 1 \mathrm{H}), 2.76-2.72(\mathrm{~m}, 1 \mathrm{H}), 1.39$ (d, $J=6.1 \mathrm{~Hz}, 3 \mathrm{H})$.

${ }^{13} \mathrm{C}$ NMR $\left(100 \mathrm{MHz}, \mathrm{CDCl}_{3}\right) \delta 198.4,139.7,137.7,137.1,136.8,129.1(2 \mathrm{C}), 128.7$ (2C), $128.6(2 \mathrm{C}), 128.5$, 128.3 (2C), 128.1 (2C), 128.0 (2C), 127.9, 127.6 (2C), 127.5 (2C), 127.2, 126.8, 76.7, 74.9, 73.1, 71.9, 66.9, $63.5,59.6,59.0,21.4$.

HRMS calculated for: $\left[\mathrm{C}_{34} \mathrm{H}_{34} \mathrm{~N}_{2} \mathrm{O}_{3}+\mathrm{H}\right]^{+} 519.2642$; found: 519.2644 . The ee was determined by UPC ${ }^{2}$ using a Chiralpak IC-3 column [1\% iPrOH $(0.5 \mathrm{~min})$, then gradient from $1 \%$ to $40 \%(10 \% / \mathrm{min}), 120 \mathrm{bar}, 40{ }^{\circ} \mathrm{C}$; flow rate $3.0 \mathrm{~mL} / \mathrm{min} ; \tau_{\text {major }}=3.85 \mathrm{~min}, \tau_{\text {minor }}=4.30 \mathrm{~min}(99 \% \mathrm{ee}) .[\alpha]^{25}{ }_{\mathrm{D}}=-115.6\left(\mathrm{c}=1.0, \mathrm{CH}_{2} \mathrm{Cl}_{2}\right.$ ). 
(3S,3'S,4R,4'R,5S,5'S)-2,2'-Dibenzyl-3,3'-bis(4-methoxyphenyl)-5-methyl-[4,5'-biisoxazolidine]-4'carbaldehyde, $5 \mathrm{~b}$<smiles>COc1ccc(C2[C@H](C=O)[C@@H]([C@H]3[C@@H](C)ON(Cc4ccccc4)[C@H]3c3ccc(OC)cc3)ON2Cc2ccccc2)cc1</smiles>

Following the general procedure (reaction time $48 \mathrm{~h}$ ) the product $\mathbf{5 b}$ was isolated by FC on silica (EtOAc/pentane 10:90 to 20:80) in 52\% yield as a colorless foam.

${ }^{1} \mathrm{H}$ NMR $\left(400 \mathrm{MHz}, \mathrm{CDCl}_{3}\right) \delta 9.73(\mathrm{~d}, J=1.9 \mathrm{~Hz}, 1 \mathrm{H}), 7.32-7.18(\mathrm{~m}, 14 \mathrm{H}), 6.90(\mathrm{~d}, J$ $=8.7 \mathrm{~Hz}, 2 \mathrm{H}), 6.79(\mathrm{~d}, J=8.7 \mathrm{~Hz}, 2 \mathrm{H}), 4.53(\mathrm{t}, J=5.9 \mathrm{~Hz}, 1 \mathrm{H}), 4.37-4.35(\mathrm{~m}, 1 \mathrm{H})$, $3.98(\mathrm{~d}, J=8.8 \mathrm{~Hz}, 1 \mathrm{H}), 3.92-3.88(\mathrm{~m}, 2 \mathrm{H}), 3.81(\mathrm{~s}, 3 \mathrm{H}), 3.80(\mathrm{~s}, 3 \mathrm{H}), 3.63(\mathrm{~d}, J=$ $14.3 \mathrm{~Hz}, 2 \mathrm{H}), 3.58-3.57(\mathrm{~m}, 1 \mathrm{H}), 3.36-3.32(\mathrm{~m}, 1 \mathrm{H}), 2.74-2.69(\mathrm{~m}, 1 \mathrm{H}), 1.40(\mathrm{~d}, J=$ $6.1 \mathrm{~Hz}, 3 \mathrm{H})$.

${ }^{13} \mathrm{C}$ NMR $\left(100 \mathrm{MHz}, \mathrm{CDCl}_{3}\right) \delta 198.6,159.7,159.0,137.9,137.0,131.4,129.1$ (2C), $128.9(2 \mathrm{C}), 128.7$ (2C), $128.5,128.3$ (2C), 128.1 (2C), 128.0 (2C), 127.1, 126.8, 114.5 (2C), 113.9 (2C), 76.7, 74.8, 72.8, 71.7, 66.8, $63.4,59.4,58.9,55.3,55.2,21.5$.

HRMS calculated for: $\left[\mathrm{C}_{36} \mathrm{H}_{38} \mathrm{~N}_{2} \mathrm{O}_{5}+\mathrm{H}\right]^{+} 579.2853$; found: 579.2861 . The ee was determined by UPC ${ }^{2}$ using a Chiralpak IC-3 column [1\% iPrOH (0.5 min), then gradient from $1 \%$ to $40 \%(10 \% / \mathrm{min}), 120$ bar, $40{ }^{\circ} \mathrm{C}$; flow rate $3.0 \mathrm{~mL} / \mathrm{min} ; \tau_{\text {major }}=4.21 \mathrm{~min}, \tau_{\text {minor }}=4.81 \mathrm{~min}(99 \% \mathrm{ee}) .[\alpha]_{\mathrm{D}}^{25}=-81.1\left(\mathrm{c}=1.0, \mathrm{CH}_{2} \mathrm{Cl}_{2}\right)$.

(3S,3'S,4R,4'R,5S,5'S)-2,2'-Dibenzyl-3,3'-bis(4-bromophenyl)-5-methyl-[4,5'-biisoxazolidine]-4'carbaldehyde, $5 c$<smiles>C[C@H]1ON(Cc2ccccc2)[C@H](c2ccc(Br)cc2)[C@@H]1[C@H]1ON(Cc2ccccc2)C(c2ccc(Br)cc2)[C@H]1C</smiles>

Following the general procedure (reaction time $24 \mathrm{~h}$ ) the product $5 \mathrm{c}$ was isolated by FC on silica (EtOAc/pentane 10:90 to $20: 80$ ) in 60\% yield as a colorless solid.

${ }^{1} \mathrm{H}$ NMR $\left(400 \mathrm{MHz}, \mathrm{CDCl}_{3}\right) \delta 9.76(\mathrm{~d}, J=1.7 \mathrm{~Hz}, 1 \mathrm{H}), 7.50(\mathrm{~d}, J=8.4 \mathrm{~Hz}, 2 \mathrm{H}), 7.32$ (d, $J=8.4 \mathrm{~Hz}, 2 \mathrm{H}), 7.29-7.19(\mathrm{~m}, 1 \mathrm{OH}), 7.14(\mathrm{t}, J=7.8 \mathrm{~Hz}, 4 \mathrm{H}), 4.55(\mathrm{dd}, J=7.0,5.7$ $\mathrm{Hz}, 1 \mathrm{H}), 4.21-4.13(\mathrm{~m}, 1 \mathrm{H}), 4.06(\mathrm{~d}, J=8.3 \mathrm{~Hz}, 1 \mathrm{H}), 3.88(\mathrm{t}, J=13.3 \mathrm{~Hz}, 2 \mathrm{H}), 3.73-$ $3.62(m, 3 H), 3.21-3.15(m, 1 H), 2.70-2.66(m, 1 H), 1.39(d, J=6.2 \mathrm{~Hz}, 3 \mathrm{H})$.

${ }^{13} \mathrm{C}$ NMR $\left(100 \mathrm{MHz}, \mathrm{CDCl}_{3}\right) \delta 197.7,138.9,137.3,136.4,136.3,132.3(2 \mathrm{C}), 131.6$ (2C), 129.5 (2C), $129.1(2 \mathrm{C}), 128.7$ (2C), 128.3 (2C), 128.3 (2C), 128.1 (2C), 127.4, 127.0, 122.5, 121.3, 77.6, $74.8,73.0,71.1,67.6,63.5,59.8,59.1,21.4$.

HRMS calculated for: $\left[\mathrm{C}_{34} \mathrm{H}_{32} \mathrm{Br}_{2} \mathrm{~N}_{2} \mathrm{O}_{3}+\mathrm{H}\right]^{+}$675.0852, 677.0832, 679.0812; found: 675.0856, 677.0839, 679.0822. The ee was determined by UPC ${ }^{2}$ using a Chiralpak IC-3 column [ $1 \%$ iPrOH ( $\left.0.5 \mathrm{~min}\right)$, then gradient from $1 \%$ to $40 \%(10 \% / \mathrm{min}), 120 \mathrm{bar}, 40{ }^{\circ} \mathrm{C}$; flow rate $3.0 \mathrm{~mL} / \mathrm{min} ; \tau_{\text {major }}=4.34 \mathrm{~min}, \tau_{\text {minor }}=5.55 \mathrm{~min}(99 \%$ ee). $[\alpha]_{D}^{25}=-88.1\left(\mathrm{c}=1.0, \mathrm{CH}_{2} \mathrm{Cl}_{2}\right)$. 
((3S,3'S,4R,4'S,5S,5'R)-2,2'-Dibenzyl-3,3'-bis(4-bromophenyl)-5-methyl-[4,5'-biisoxazolidin]-4'yl)methanol, 5c'

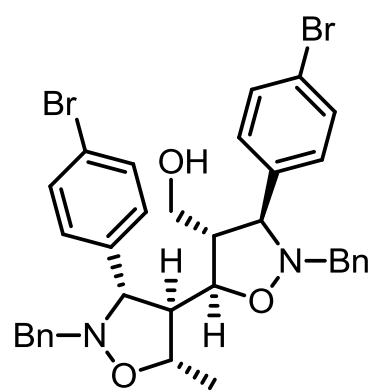

To a solution of aldehyde $5 \mathrm{c}\left(135.3 \mathrm{mg}, 0.2 \mathrm{mmol}, 1.0\right.$ equiv) in $\mathrm{CHCl}_{3}: \mathrm{MeOH}$ (1:1, $1.0 \mathrm{~mL}$ ), $\mathrm{NaBH}_{4}$ (15.1 mg, $0.4 \mathrm{mmol}, 2.0$ equiv) was added. The reaction mixture was stirred at room temperature for $1 \mathrm{~h}$. The mixture was diluted with $\mathrm{CH}_{2} \mathrm{Cl}_{2}$, sat. aq. $\mathrm{NH}_{4} \mathrm{Cl}$ was added, and the aqueous phase was extracted with $\mathrm{CH}_{2} \mathrm{Cl}_{2}$. The combined organic phase was dried over $\mathrm{MgSO}_{4}$, filtered and the solvent was removed to afford alcohol $\mathbf{5} \mathbf{c}^{\prime}$ as a colorless foam in quantitative yield.

${ }^{1} \mathrm{H}$ NMR $\left(400 \mathrm{MHz}, \mathrm{CDCl}_{3}\right) \delta 7.46(\mathrm{~d}, J=8.4 \mathrm{~Hz}, 2 \mathrm{H}), 7.28-7.17(\mathrm{~m}, 12 \mathrm{H}), 7.11(\mathrm{t}, J$ $=6.8 \mathrm{~Hz}, 4 \mathrm{H}), 4.33-4.25(\mathrm{~m}, 1 \mathrm{H}), 4.18(\mathrm{dd}, J=7.4,4.9 \mathrm{~Hz}, 1 \mathrm{H}), 3.87(\mathrm{~d}, J=4.1 \mathrm{~Hz}, 1 \mathrm{H}), 3.82(\mathrm{~d}, J=4.4 \mathrm{~Hz}, 1 \mathrm{H})$, 3.80-3.65 (m, 4H), $3.61(\mathrm{~d}, J=14.0 \mathrm{~Hz}, 1 \mathrm{H}), 3.49(\mathrm{~d}, J=8.4 \mathrm{~Hz}, 1 \mathrm{H}), 2.70-2.64(\mathrm{~m}, 1 \mathrm{H}), 2.38-2.31(\mathrm{~m}, 1 \mathrm{H})$, 1.45 (bs, $1 \mathrm{H}), 1.37(\mathrm{~d}, J=6.1 \mathrm{~Hz}, 3 \mathrm{H})$.

${ }^{13} \mathrm{C}$ NMR $\left(100 \mathrm{MHz}, \mathrm{CDCl}_{3}\right) \delta 140.3,137.7(2 \mathrm{C}), 137.0,132.0(2 \mathrm{C}), 131.4(2 \mathrm{C}), 129.5(2 \mathrm{C}), 129.4(2 \mathrm{C}), 128.8$ (2C), 128.4 (2C), 128.1 (2C), 128.1 (2C), 127.2, 126.9, 121.9, 120.8, 81.1, 75.0, 73.6, 72.9, 63.8, 62.5, 60.2, $59.4,58.3,20.9$.

HRMS calculated for: $\left[\mathrm{C}_{34} \mathrm{H}_{34} \mathrm{Br}_{2} \mathrm{~N}_{2} \mathrm{O}_{3}+\mathrm{H}\right]^{+}$677.1009, 679.0988, 681.0968; found: 677.1007, 679.0989, 681.0978. The ee was determined by UPC ${ }^{2}$ using a Chiralpak IC-3 column [ $1 \%$ iPrOH ( $\left.0.5 \mathrm{~min}\right)$, then gradient from $1 \%$ to $40 \%(10 \% / \mathrm{min}), 120 \mathrm{bar}, 40{ }^{\circ} \mathrm{C}$; flow rate $3.0 \mathrm{~mL} / \mathrm{min} ; \tau_{\text {major }}=4.18 \mathrm{~min}, \tau_{\text {minor }}=4.52 \mathrm{~min}(99 \%$ ee). $[\alpha]_{D}^{25}=-81.8\left(\mathrm{c}=1.0, \mathrm{CH}_{2} \mathrm{Cl}_{2}\right)$.

(3S,3'S,4R,4'R,5S,5'S)-2,2'-Dibenzyl-3,3'-bis(2-methoxyphenyl)-5-methyl-[4,5'-biisoxazolidine]-4'carbaldehyde, $5 \mathrm{~d}$<smiles>COc1ccccc1C1[C@H](C=O)[C@@H]([C@H]2[C@@H](C)ON(Cc3ccccc3)[C@H]2c2ccccc2OC)ON1Cc1ccccc1</smiles>

Following the general procedure (reaction time $3 \mathrm{~d}$ ) the product $\mathbf{5 d}$ was isolated by FC on silica (EtOAc/pentane 10:90 to $20: 80$ ) in $60 \%$ yield as a yellow oil.

${ }^{1} \mathrm{H} \mathrm{NMR}\left(400 \mathrm{MHz}, \mathrm{CDCl}_{3}\right) \delta 9.90(\mathrm{~d}, J=3.2 \mathrm{~Hz}, 1 \mathrm{H}), 7.63$ (dd, $J=7.6,1.3 \mathrm{~Hz}$, $1 \mathrm{H}), 7.60(\mathrm{dd}, J=7.6,1.3 \mathrm{~Hz}, 1 \mathrm{H}), 7.35-7.14(\mathrm{~m}, 12 \mathrm{H}), 6.95(\mathrm{td}, J=13.6,0.8$ $\mathrm{Hz}, 2 \mathrm{H}), 6.83(\mathrm{dd}, J=11.1,8.3 \mathrm{~Hz}, 2 \mathrm{H}), 4.70(\mathrm{dd}, J=6.4,4.1 \mathrm{~Hz}, 1 \mathrm{H}), 4.56$ (d, $J=6.4 \mathrm{~Hz}, 1 \mathrm{H}), 4.23-4.18(\mathrm{~m}, 1 \mathrm{H}), 4.09(\mathrm{~d}, J=13.8 \mathrm{~Hz}, 1 \mathrm{H}), 3.98-3.85(\mathrm{~m}, 3 \mathrm{H}), 3.80(\mathrm{~s}, 3 \mathrm{H}), 3.74(\mathrm{~s}, 3 \mathrm{H}), 3.65$ (d, $J=14.8 \mathrm{~Hz}, 1 \mathrm{H}), 3.43-3.39(\mathrm{~m}, 1 \mathrm{H}), 2.67-2.63(\mathrm{~m}, 1 \mathrm{H}), 1.37(\mathrm{~d}, J=6.1 \mathrm{~Hz}, 3 \mathrm{H})$.

${ }^{13} \mathrm{C} \mathrm{NMR}\left(100 \mathrm{MHz}, \mathrm{CDCl}_{3}\right) \delta 199.5,157.1,156.2,138.1,137.3,128.6$ (2C), 128.5, 128.4, 128.2 (2C), 128.1 (3C), 128.0 (2C), 127.5, 127.2, 127.1, 126.7, 126.7, 121.1, 120.9, 110.5, 110.3, 77.8, 74.8, 66.1, 65.6, 65.3, $62.0,60.1,58.9,55.1,55.0,21.9$.

HRMS calculated for: $\left[\mathrm{C}_{36} \mathrm{H}_{38} \mathrm{~N}_{2} \mathrm{O}_{5}+\mathrm{H}\right]^{+} 579.2853$; found: 579.2856 . The ee was determined by UPC ${ }^{2}$ using a Chiralpak IC-3 column [1\% MeCN (0.5 min), then gradient from $1 \%$ to $40 \%(2 \% / \mathrm{min}), 120 \mathrm{bar}, 40{ }^{\circ} \mathrm{C}$; flow rate $3.0 \mathrm{~mL} / \mathrm{min} ; \tau_{\text {major }}=8.08 \mathrm{~min}, \tau_{\text {minor }}=8.87 \mathrm{~min}(99 \% \mathrm{ee}) .[\alpha]_{\mathrm{D}}^{25}=-87.6\left(\mathrm{c}=1.0, \mathrm{CH}_{2} \mathrm{Cl}_{2}\right)$. 
<smiles>Cc1cccc(C2[C@H](C=O)[C@@H]([C@H]3[C@@H](C)ON(Cc4ccccc4)[C@H]3c3cccc(C)c3)ON2Cc2ccccc2)c1</smiles>

Following the general procedure (reaction time $24 \mathrm{~h}$ ) the product $5 \mathrm{e}$ was isolated by FC on silica (EtOAc/pentane $5: 90$ to 10:90) in 60\% yield as a yellow oil.

${ }^{1} \mathrm{H}$ NMR $\left(400 \mathrm{MHz}, \mathrm{CDCl}_{3}\right) \delta 9.74(\mathrm{~d}, J=1.9 \mathrm{~Hz}, 1 \mathrm{H}), 7.30-7.02(\mathrm{~m}, 18 \mathrm{H}), 4.56$ $(\mathrm{t}, J=5.7 \mathrm{~Hz}, 1 \mathrm{H}), 4.41-4.30(\mathrm{~m}, 1 \mathrm{H}), 4.03(\mathrm{~d}, J=8.6 \mathrm{~Hz}, 1 \mathrm{H}), 3.93(\mathrm{~d}, J=14.1$ $\mathrm{Hz}, 1 \mathrm{H}), 3.88(\mathrm{~d}, J=14.6 \mathrm{~Hz}, 1 \mathrm{H}), 3.68(\mathrm{~d}, J=14.1 \mathrm{~Hz}, 1 \mathrm{H}), 3.66-3.60(\mathrm{~m}, 2 \mathrm{H})$, $3.38(\mathrm{ddd}, J=8.3,6.2,1.8 \mathrm{~Hz}, 1 \mathrm{H}), 2.72-2.70(\mathrm{~m}, 1 \mathrm{H}), 2.36(\mathrm{~s}, 3 \mathrm{H}), 2.32(\mathrm{~s}, 3 \mathrm{H}), 1.40(\mathrm{~d}, J=6.1 \mathrm{~Hz}, 3 \mathrm{H})$.

${ }^{13} \mathrm{C} \mathrm{NMR}\left(100 \mathrm{MHz}, \mathrm{CDCl}_{3}\right) \delta 198.6,139.6,138.8,138.1,137.8,137.0,136.9,129.3,129.0,128.7(2 \mathrm{C}), 128.6$, $128.5,128.4,128.3(2 \mathrm{C}), 128.2,128.1$ (2C), 128.0 (2C), 127.2, 126.8, 125.2, 124.5, 76.4, 74.9, 73.0, 71.9, $66.6,63.2,59.6,59.1,21.5,21.5,21.2$.

HRMS calculated for: $\left[\mathrm{C}_{36} \mathrm{H}_{38} \mathrm{~N}_{2} \mathrm{O}_{3}+\mathrm{H}\right]^{+} 547.2955$; found: 547.2963 . The ee was determined by UPC ${ }^{2}$ using a Chiralpak IC-3 column [1\% iPrOH (0.5 min), then gradient from $1 \%$ to $40 \%(2 \% / \mathrm{min}), 120 \mathrm{bar}, 40{ }^{\circ} \mathrm{C}$; flow rate $3.0 \mathrm{~mL} / \mathrm{min} ; \tau_{\text {major }}=8.32 \mathrm{~min}, \tau_{\text {minor }}=9.32 \mathrm{~min}(99 \% \mathrm{ee}) .[\alpha]_{\mathrm{D}}^{25}=-93.2\left(\mathrm{c}=1.0, \mathrm{CH}_{2} \mathrm{Cl}_{2}\right)$.

\section{(3S,3'S,4R,4'R,5S,5'S)-2,2'-Dibenzyl-5-methyl-3,3'-di(naphthalen-2-yl)-[4,5'-biisoxazolidine]-4'-}<smiles>C[C@@H]1ON(Cc2ccccc2)[C@H](c2ccc3ccccc3c2)[C@@H]1[C@H]1ON(Cc2ccccc2)[C@H](c2ccc3ccccc3c2)[C@@H]1C=O</smiles>

Following the general procedure (reaction time $24 \mathrm{~h}$ ) the product $\mathbf{5 f}$ was isolated by FC on silica (EtOAc/pentane $8: 92$ to $20: 80$ ) in $61 \%$ yield as a colorless foam.

${ }^{1} \mathrm{H}$ NMR $\left(400 \mathrm{MHz}, \mathrm{CDCl}_{3}\right) \delta 9.79(\mathrm{~d}, J=1.7 \mathrm{~Hz}, 1 \mathrm{H}), 7.86-7.70(\mathrm{~m}, 10 \mathrm{H}), 7.54-$ $7.44(\mathrm{~m}, 8 \mathrm{H}), 7.29-7.10(\mathrm{~m}, 6 \mathrm{H}), 4.67(\mathrm{t}, J=5.9 \mathrm{~Hz}, 1 \mathrm{H}), 4.43-4.35(\mathrm{~m}, 1 \mathrm{H}), 4.25$ (d, $J=8.5 \mathrm{~Hz}, 1 \mathrm{H}), 3.97-3.87(\mathrm{~m}, 3 \mathrm{H}), 3.78-3.70(\mathrm{~m}, 2 \mathrm{H}), 3.46$ (ddd, $J=8.0,6.0$, $1.7 \mathrm{~Hz}, 1 \mathrm{H}), 2.90-2.85(\mathrm{~m}, 1 \mathrm{H}), 1.46(\mathrm{~d}, J=6.1 \mathrm{~Hz}, 3 \mathrm{H})$.

${ }^{13} \mathrm{C} \mathrm{NMR}\left(100 \mathrm{MHz}, \mathrm{CDCl}_{3}\right) \delta 198.3,137.7,137.2,136.7,134.5,133.3,133.3,133.3,133.0,129.2,128.7(2 \mathrm{C})$, $128.5,128.4(2 \mathrm{C}), 128.1$ (2C), 128.1 (2C), 127.9, 127.9, 127.7, 127.7, 127.3, 127.2, 127.2, 126.9, 126.5, $126.5,126.0,125.9,125.7,124.6,76.9,75.1,73.3,71.0,66.9,63.2,59.7,59.2,21.2$.

HRMS calculated for: $\left[\mathrm{C}_{42} \mathrm{H}_{38} \mathrm{~N}_{2} \mathrm{O}_{3}+\mathrm{H}\right]^{+} 619.2955$; found: 619.2959 . The ee was determined by UPC ${ }^{2}$ using a Chiralpak IC-3 column [1\% iPrOH (0.5 min), then gradient from $1 \%$ to $40 \%(10 \% / \mathrm{min}), 120 \mathrm{bar}, 40{ }^{\circ} \mathrm{C}$; flow rate $3.0 \mathrm{~mL} / \mathrm{min} ; \tau_{\text {major }}=4.93 \mathrm{~min}, \tau_{\text {minor }}=5.90 \mathrm{~min}(99 \% \mathrm{ee}) .[\alpha]_{\mathrm{D}}^{25}=-57.8\left(\mathrm{c}=1.0, \mathrm{CH}_{2} \mathrm{Cl}_{2}\right)$. 
<smiles>C[C@@H]1ON(C)[C@@H](c2ccccc2)[C@H]1[C@@H]1ON(C)[C@H](C=O)[C@@H]1c1ccccc1</smiles>

Following the general procedure (reaction time $3 \mathrm{~d}$ ) the product $\mathbf{5 g}$ was isolated by FC on silica (EtOAc/pentane 5:95 to 10:90) in 56\% yield as a yellow oil.

${ }^{1} \mathrm{H}$ NMR $\left(400 \mathrm{MHz}, \mathrm{CDCl}_{3}\right) \delta 9.75(\mathrm{~d}, J=2.1 \mathrm{~Hz}, 1 \mathrm{H}), 7.43-7.41(\mathrm{~m}, 2 \mathrm{H}), 7.36-7.28$ $(\mathrm{m}, 8 \mathrm{H}), 4.50(\mathrm{t}, J=6.0 \mathrm{~Hz}, 1 \mathrm{H}), 4.26-4.21(\mathrm{~m}, 1 \mathrm{H}), 3.77(\mathrm{~d}, J=8.6 \mathrm{~Hz}, 1 \mathrm{H}), 3.49$ (bs, $1 \mathrm{H}), 3.27-3.23(\mathrm{~m}, 1 \mathrm{H}), 2.86-2.81(\mathrm{~m}, 1 \mathrm{H}), 2.58(\mathrm{~s}, 3 \mathrm{H}), 2.45(\mathrm{~s}, 3 \mathrm{H}), 1.49(\mathrm{~d}, J$ $=6.2 \mathrm{~Hz}, 3 \mathrm{H})$.

${ }^{13} \mathrm{C} \mathrm{NMR}\left(100 \mathrm{MHz}, \mathrm{CDCl}_{3}\right) \delta 198.3,139.2,136.8,129.0$ (2C), 128.5 (3C), $128.1(2 \mathrm{C}), 127.7,127.5$ (2C), 77.0 (2C), 74.9, 74.4, 67.8, 63.7, 43.2, 42.6, 22.0.

HRMS calculated for: $\left[\mathrm{C}_{22} \mathrm{H}_{26} \mathrm{~N}_{2} \mathrm{O}_{3}+\mathrm{H}\right]^{+} 367.2016$; found: 367.2024 . The ee was determined by UPC ${ }^{2}$ using a Chiralpak IC-3 column [1\% iPrOH (0.5 min), then gradient from $1 \%$ to $40 \%(10 \% / \mathrm{min}), 120$ bar, $40{ }^{\circ} \mathrm{C}$; flow rate $3.0 \mathrm{~mL} / \mathrm{min} ; \tau_{\text {major }}=3.17 \mathrm{~min}, \tau_{\text {minor }}=3.00 \mathrm{~min}(92 \%$ ee $) .[\alpha]^{25}=-137.2\left(\mathrm{c}=1.0, \mathrm{CH}_{2} \mathrm{Cl}_{2}\right)$.

\section{(3S,3'S,4R,4'R,5S,5'S)-5-Methyl-2,2',3,3'-tetraphenyl-[4,5'-biisoxazolidine]-4'-carbaldehyde, 5h}<smiles>C[C@@H]1ON(c2ccccc2)[C@H](c2ccccc2)[C@H]1[C@H]1ON(c2ccccc2)[C@H](c2ccccc2)[C@H]1C=O</smiles>

Following the general procedure (reaction time $15 \mathrm{~h}$ ) the product $5 \mathrm{~h}$ was isolated by FC on silica (EtOAc/pentane 8:92 to $15: 85$ ) in $86 \%$ yield as a colorless foam.

${ }^{1} \mathrm{H}$ NMR $\left(400 \mathrm{MHz}, \mathrm{CDCl}_{3}\right) \delta 9.37(\mathrm{~d}, J=2.2 \mathrm{~Hz}, 1 \mathrm{H}), 7.35(\mathrm{~d}, J=7.4 \mathrm{~Hz}, 2 \mathrm{H}), 7.31-$ $7.04(\mathrm{~m}, 10 \mathrm{H}), 6.90-6.73(\mathrm{~m}, 8 \mathrm{H}), 4.93(\mathrm{~d}, J=4.9 \mathrm{~Hz}, 1 \mathrm{H}), 4.86(\mathrm{~d}, J=4.4 \mathrm{~Hz}, 1 \mathrm{H})$, $4.39(\mathrm{t}, J=6.9 \mathrm{~Hz}, 1 \mathrm{H}), 4.05-3.96(\mathrm{~m}, 1 \mathrm{H}), 3.06-3.02(\mathrm{~m}, 1 \mathrm{H}), 2.71-2.67(\mathrm{~m}, 1 \mathrm{H})$, $1.24(\mathrm{~d}, J=6.0 \mathrm{~Hz}, 3 \mathrm{H})$.

${ }^{13} \mathrm{C} \mathrm{NMR}\left(100 \mathrm{MHz}, \mathrm{CDCl}_{3}\right) \delta 197.3,151.0,149.5,142.5,139.9,129.1(2 \mathrm{C}), 129.1(2 \mathrm{C}), 129.0(2 \mathrm{C}), 128.8(2 \mathrm{C})$, 128.0, 127.3, $126.6(2 \mathrm{C}), 126.2(2 \mathrm{C}), 122.8,121.6,115.0(2 \mathrm{C}), 114.1(2 \mathrm{C}), 78.1,76.3,72.9,70.9,68.8,63.1$, 18.6 .

HRMS calculated for: $\left[\mathrm{C}_{32} \mathrm{H}_{30} \mathrm{~N}_{2} \mathrm{O}_{3}+\mathrm{H}\right]^{+}$491.2329; found: 491.2334. The ee was determined by $\mathrm{UPC}^{2}$ after reduction to the alcohol $5 h^{\prime}\left(99 \%\right.$ ee). $[\alpha]^{25}=-147.8\left(\mathrm{c}=1.0, \mathrm{CH}_{2} \mathrm{Cl}_{2}\right)$.

\section{((3S,3'S,4R,4'S,5S,5'R)-5-Methyl-2,2',3,3'-tetraphenyl-[4,5'-biisoxazolidin]-4'-yl)methanol, 5h'}<smiles>C[C@@H]1ON(c2ccccc2)[C@@H](c2ccccc2)[C@H]1[C@H]1ON(c2ccccc2)[C@H](c2ccccc2)[C@H]1CO</smiles>

To a solution of aldehyde $5 \mathrm{~h}$ ( $42.3 \mathrm{mg}, 0.086$ mmol, 1.0 equiv) in $\mathrm{CHCl}_{3}: \mathrm{MeOH}$ (1:1, $0.4 \mathrm{~mL}), \mathrm{NaBH}_{4}(6.5 \mathrm{mg}, 0.17 \mathrm{mmol}, 2.0$ equiv) was added. The reaction mixture was stirred at room temperature for $1 \mathrm{~h}$. The mixture was diluted with $\mathrm{CH}_{2} \mathrm{Cl}_{2}$, sat. aq. $\mathrm{NH}_{4} \mathrm{Cl}$ was added, and the aqueous phase was extracted with $\mathrm{CH}_{2} \mathrm{Cl}_{2}$. The combined organic phase was dried over $\mathrm{MgSO}_{4}$, filtered and the solvent was removed to afford alcohol $\mathbf{5} \mathbf{h}^{\prime}$ as a colorless foam in $61 \%$ yield. 
${ }^{1} \mathrm{H}$ NMR $\left(400 \mathrm{MHz}, \mathrm{CDCl}_{3}\right)$ 8 7.48-7.35 (m, 6H), 7.35-7.15 (m, 8H), 7.02 (d, J= 7.8 Hz, 2H), 6.93 $(\mathrm{t}, J=7.3 \mathrm{~Hz}$, $2 \mathrm{H}), 6.86(\mathrm{~d}, J=7.8 \mathrm{~Hz}, 2 \mathrm{H}), 5.05(\mathrm{~d}, J=4.8 \mathrm{~Hz}, 1 \mathrm{H}), 4.47(\mathrm{~d}, J=5.1 \mathrm{~Hz}, 1 \mathrm{H}), 4.32-4.23(\mathrm{~m}, 1 \mathrm{H}), 4.07(\mathrm{t}, J=6.2$ $\mathrm{Hz}, 1 \mathrm{H}), 3.60-3.47(\mathrm{~m}, 2 \mathrm{H}), 2.88-2.80(\mathrm{~m}, 1 \mathrm{H}), 2.54-2.47(\mathrm{~m}, 1 \mathrm{H}), 1.38(\mathrm{~d}, J=6.0 \mathrm{~Hz}, 3 \mathrm{H})$.

${ }^{13} \mathrm{C} \mathrm{NMR}\left(100 \mathrm{MHz}, \mathrm{CDCl}_{3}\right) \delta 150.5,149.5,142.3,140.4,127.9(2 \mathrm{C}), 127.9(2 \mathrm{C}), 127.8(2 \mathrm{C}), 127.7(2 \mathrm{C}), 126.6$, $126.1,125.8$ (2C), 125.4 (2C), 121.0, 120.2, 114.0 (2C), 113.1 (2C), 78.9, 75.4, 71.4, 71.4, 62.3, 61.7, 58.2, 17.5.

HRMS calculated for: $\left[\mathrm{C}_{32} \mathrm{H}_{32} \mathrm{~N}_{2} \mathrm{O}_{3}+\mathrm{H}\right]^{+}$493.2486; found: 493.2494. The ee was determined by UPC ${ }^{2}$ using a Chiralpak IB-3 column [1\% MeOH (0.5 min), then gradient from $1 \%$ to $40 \%(10 \% / \mathrm{min}), 120 \mathrm{bar}, 40{ }^{\circ} \mathrm{C}$; flow rate $3.0 \mathrm{~mL} / \mathrm{min} ; \tau_{\text {major }}=3.91 \mathrm{~min}, \tau_{\text {minor }}=4.29 \mathrm{~min}(99 \%$ ee $) .[\alpha]^{25}{ }_{\mathrm{D}}=-161.0\left(\mathrm{c}=1.0, \mathrm{CH}_{2} \mathrm{Cl}_{2}\right.$ ).

\section{(3S,3'S,4R,4'R,5S,5'S)-2,2'-Dibenzyl-5-hexyl-3,3'-diphenyl-[4,5'-biisoxazolidine]-4'-carbaldehyde, $5 i$}<smiles>O=C[C@@H]1ON(Cc2ccccc2)[C@@H](c2ccccc2)[C@@H]1[C@H]1[C@@H](C=O)[C@@H](c2ccccc2)ON1Cc1ccccc1</smiles>

Following the general procedure (reaction time $24 \mathrm{~h}$ ) the product $\mathbf{5 i}$ was isolated by FC on silica (EtOAc/pentane 5:95 to 10:90) in $47 \%$ yield as a yellow oil.

${ }^{1} \mathrm{H} \mathrm{NMR}\left(400 \mathrm{MHz}, \mathrm{CDCl}_{3}\right) \delta 9.78(\mathrm{~d}, J=1.8 \mathrm{~Hz}, 1 \mathrm{H}), 7.41-7.17(\mathrm{~m}, 20 \mathrm{H}), 4.57(\mathrm{t}, J=$ $5.8 \mathrm{~Hz}, 1 \mathrm{H}), 4.14-4.07(\mathrm{~m}, 1 \mathrm{H}), 4.08(\mathrm{~d}, J=8.5 \mathrm{~Hz}, 1 \mathrm{H}), 3.93-3.89(\mathrm{~m}, 2 \mathrm{H}), 3.69(\mathrm{~d}, J$ $=13.9 \mathrm{~Hz}, 1 \mathrm{H}), 3.64(\mathrm{~d}, J=14.8 \mathrm{~Hz}, 1 \mathrm{H}), 3.53(\mathrm{~d}, J=7.2 \mathrm{~Hz}, 1 \mathrm{H}), 3.42-3.38(\mathrm{~m}, 1 \mathrm{H})$, 2.78-2.74 (m, $1 \mathrm{H}), 1.99-1.90(\mathrm{~m}, 1 \mathrm{H}), 1.50-1.43(\mathrm{~m}, 1 \mathrm{H}), 1.38-1.25(\mathrm{~m}, 8 \mathrm{H}), 0.89(\mathrm{t}$, $J=6.8 \mathrm{~Hz}, 3 \mathrm{H})$.

${ }^{13} \mathrm{C} \mathrm{NMR}\left(100 \mathrm{MHz}, \mathrm{CDCl}_{3}\right) \delta 198.5,139.4,137.9,137.3,136.8,129.1(2 \mathrm{C}), 128.8(2 \mathrm{C}), 128.6(2 \mathrm{C}), 128.5$, $128.2(2 \mathrm{C}), 128.1(2 \mathrm{C}), 128.0(4 \mathrm{C}), 127.6,127.6$ (2C), 127.2, 126.7, 79.0, 76.9, 73.1, 71.9, 66.9, 62.1, 59.7, $58.9,35.9,31.8,29.2,26.2,22.6,14.1$.

HRMS calculated for: $\left[\mathrm{C}_{39} \mathrm{H}_{44} \mathrm{~N}_{2} \mathrm{O}_{3}+\mathrm{H}\right]^{+}$589.3425; found: 589.3431 . The ee was determined by $\mathrm{UPC}^{2}$ using a Chiralpak ID-3 column [1\% iPrOH (0.5 min), then gradient from $1 \%$ to $40 \%(5 \% / \mathrm{min}), 120 \mathrm{bar}, 40{ }^{\circ} \mathrm{C}$; flow rate $3.0 \mathrm{~mL} / \mathrm{min} ; \tau_{\text {major }}=4.67 \mathrm{~min}, \tau_{\text {minor }}=4.94 \mathrm{~min}(99 \% \mathrm{ee}) .[\alpha]_{\mathrm{D}}^{25}=-81.4\left(\mathrm{c}=1.0, \mathrm{CH}_{2} \mathrm{Cl}_{2}\right.$ ).

\section{(3S,3'S,4R,4'R,5S,5'S)-2,2'-Dibenzyl-5-phenethyl-3,3'-diphenyl-[4,5'-biisoxazolidine]-4'-carbaldehyde, 5j}

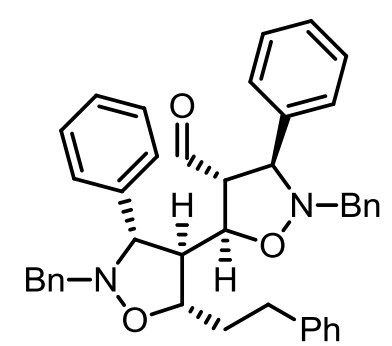

Following the general procedure (reaction time $24 \mathrm{~h}$ ) the product $5 \mathbf{j}$ was isolated by FC on silica (EtOAc/pentane 10:90) in $72 \%$ yield as a yellow oil.

${ }^{1} \mathrm{H}$ NMR $\left(400 \mathrm{MHz}, \mathrm{CDCl}_{3}\right) \delta 9.74(\mathrm{~d}, J=1.7 \mathrm{~Hz}, 1 \mathrm{H}), 7.39-7.12(\mathrm{~m}, 25 \mathrm{H}), 4.56(\mathrm{t}, J=$ $5.7 \mathrm{~Hz}, 1 \mathrm{H}), 4.24-4.20(\mathrm{~m}, 1 \mathrm{H}), 4.05(\mathrm{~d}, J=8.7 \mathrm{~Hz}, 1 \mathrm{H}), 3.92(\mathrm{~d}, J=14.7 \mathrm{~Hz}, 1 \mathrm{H})$, $3.88(\mathrm{~d}, J=14.0 \mathrm{~Hz}, 1 \mathrm{H}), 3.64(\mathrm{~d}, J=13.9 \mathrm{~Hz}, 1 \mathrm{H}), 3.63(\mathrm{~d}, J=14.8 \mathrm{~Hz}, 1 \mathrm{H}), 3.48(\mathrm{~d}$, $J=7.5 \mathrm{~Hz}, 1 \mathrm{H}), 3.43-3.39(\mathrm{~m}, 1 \mathrm{H}), 2.81-2.77(\mathrm{~m}, 1 \mathrm{H}), 2.71-2.64(\mathrm{~m}, 1 \mathrm{H}), 2.58-2.50$ (m, 1H), 2.34-2.25 (m, 1H), 1.81-1.72 (m, 1H). 
${ }^{13} \mathrm{C}$ NMR $\left(100 \mathrm{MHz}, \mathrm{CDCl}_{3}\right) \delta 198.5,142.1,139.0,138.0,137.1,136.8,129.2(2 \mathrm{C}), 128.8(2 \mathrm{C}), 128.7(2 \mathrm{C})$, $128.6(2 \mathrm{C}), 128.5,128.3(2 \mathrm{C}), 128.2$ (2C), $128.2(2 \mathrm{C}), 128.0(2 \mathrm{C}), 127.9(2 \mathrm{C}), 127.8,127.5(2 \mathrm{C}), 127.2,126.9$, $125.7,77.9,76.5,73.3,72.0,66.6,62.3,59.7,58.9,37.7,32.5$.

HRMS calculated for: $\left[\mathrm{C}_{41} \mathrm{H}_{40} \mathrm{~N}_{2} \mathrm{O}_{3}+\mathrm{H}\right]^{+}$609.3112; found: 609.3113 . The ee was determined by $\mathrm{UPC}^{2}$ using a Chiralpak ID-3 column [1\% iPrOH (0.5 min), then gradient from $1 \%$ to $40 \%(10 \% / \mathrm{min}), 120 \mathrm{bar}, 40{ }^{\circ} \mathrm{C}$; flow rate $3.0 \mathrm{~mL} / \mathrm{min} ; \tau_{\text {major }}=4.13 \mathrm{~min}, \tau_{\text {minor }}=4.31 \mathrm{~min}(99 \% \mathrm{ee}) .[\alpha]_{\mathrm{D}}^{25}=-87.1\left(\mathrm{c}=1.0, \mathrm{CH}_{2} \mathrm{Cl}_{2}\right)$. 


\section{Functionalization of isoxazolidine-substituted enal $4 a$}

\subsection{General procedure for the organocatalytic synthesis of $6 a, b$}

A glass vial equipped with a magnetic stirring bar was charged with catalyst $3 c$ or ent-3c $(15.7 \mathrm{mg}, 0.02$ mmol, 0.2 equiv), nitrone $2 \mathrm{~m}\left(0.12 \mathrm{mmol}, 1.2\right.$ equiv), and dry $\mathrm{CHCl}_{3}(0.45 \mathrm{~mL})$. Aq. sat. $\mathrm{KCl}(5.4 \mu \mathrm{L}, 0.025$ $\mathrm{mmol}, 0.25$ equiv) was added followed by addition of $4 \mathrm{a}(32.1 \mathrm{mg}, 0.1 \mathrm{mmol}, 1.0$ equiv). A solution of TfOH in $\mathrm{CHCl}_{3}(50 \mu \mathrm{L}, 0.005 \mathrm{mmol}, 0.05$ equiv, $0.1 \mathrm{M}$ ) was added and the reaction stirred at room temperature until completion. The diastereomeric ratio was determined by ${ }^{1} \mathrm{H} N M R$ analysis of the crude reaction mixture by comparing the integrals of the peaks corresponding to the aldehyde protons of the diastereoisomeric products.

\subsection{Results and characterization}

(3S,3'S,4R,4'R,5S,5'S)-2,2'-Dibenzyl-5-methyl-3'-(naphthalen-2-yl)-3-(o-tolyl)-[4,5'-biisoxazolidine]-4'carbaldehyde, $6 \mathrm{a}$

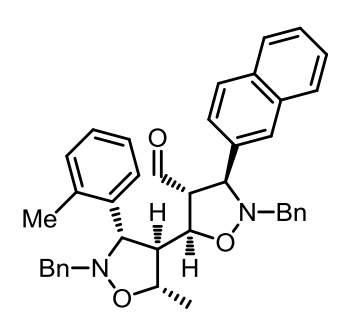

Following the general procedure applying catalyst 3c (reaction time $48 \mathrm{~h}$ ) the product 6a was isolated by FC on silica (EtOAc/pentane $5: 95$ to 10:90) in $50 \%$ yield as a colorless foam.

${ }^{1} \mathrm{H} \mathrm{NMR}\left(400 \mathrm{MHz}, \mathrm{CDCl}_{3}\right) \delta 9.80(\mathrm{~d}, J=2.1 \mathrm{~Hz}, 1 \mathrm{H}), 7.85-7.78(\mathrm{~m}, 4 \mathrm{H}), 7.60(\mathrm{~d}, J=7.5$ $\mathrm{Hz}, 1 \mathrm{H}), 7.53-7.45(\mathrm{~m}, 3 \mathrm{H}), 7.29-7.08(\mathrm{~m}, 12 \mathrm{H}), 7.01(\mathrm{~d}, J=7.5 \mathrm{~Hz}, 1 \mathrm{H}), 4.64(\mathrm{t}, J=6.3$ $\mathrm{Hz}, 1 \mathrm{H}), 4.27-4.23(\mathrm{~m}, 2 \mathrm{H}), 4.00(\mathrm{bs}, 1 \mathrm{H}), 3.92(\mathrm{~d}, J=14.3 \mathrm{~Hz}, 1 \mathrm{H}), 3.86(\mathrm{~d}, J=14.4 \mathrm{~Hz}$,

$1 \mathrm{H}), 3.72(\mathrm{~d}, J=14.2 \mathrm{~Hz}, 1 \mathrm{H}), 3.65(\mathrm{~d}, J=14.2 \mathrm{~Hz}, 1 \mathrm{H}), 3.42-3.38(\mathrm{~m}, 1 \mathrm{H}), 2.96-2.91(\mathrm{~m}, 1 \mathrm{H}), 2.16(\mathrm{bs}, 3 \mathrm{H})$, $1.48(\mathrm{~d}, J=6.2 \mathrm{~Hz}, 3 \mathrm{H})$.

${ }^{13} \mathrm{C} \mathrm{NMR}\left(100 \mathrm{MHz}, \mathrm{CDCl}_{3}\right) \delta 198.2,137.9,137.1,136.8,136.6,134.4,133.3,133.3,130.7,129.1,128.5$ (2C), $128.4,128.2$ (2C), 128.1 (2C), 128.1 (2C), 127.8, 127.7, 127.3, 127.1, 127.0, 126.8, 126.5, 126.4, 126.2, $124.6,78.0,75.1,72.0,70.5,67.4,63.0,59.6,58.8,21.5,19.6$.

HRMS calculated for: $\left[\mathrm{C}_{39} \mathrm{H}_{38} \mathrm{~N}_{2} \mathrm{O}_{3}+\mathrm{H}\right]^{+} 583.2955$; found: 583.2960 . The ee was determined by UPC ${ }^{2}$ using a Chiralpak IC-3 column [ $1 \% \mathrm{MeCN}(0.5 \mathrm{~min})$, then gradient from $1 \%$ to $40 \%(10 \% / \mathrm{min}), 120 \mathrm{bar}, 40{ }^{\circ} \mathrm{C}$; flow rate $3.0 \mathrm{~mL} / \mathrm{min} ; \tau_{\text {major }}=4.45 \mathrm{~min}, \tau_{\text {minor }}=5.49 \min (99 \% \mathrm{ee}) .[\alpha]_{\mathrm{D}}^{25}=-94.7\left(\mathrm{c}=1.0, \mathrm{CH}_{2} \mathrm{Cl}_{2}\right)$. 
(3S,3'R,4R,4'S,5S,5' R)-2,2'-Dibenzyl-5-methyl-3'-(naphthalen-2-yl)-3-(o-tolyl)-[4,5'-biisoxazolidine]-4'carbaldehyde, $6 \mathrm{~b}$

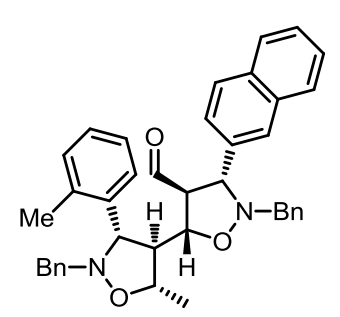

Following the general procedure applying catalyst ent-3c (reaction time $48 \mathrm{~h}$ ) the product $\mathbf{6} \mathbf{b}$ was isolated by FC on silica (EtOAc/pentane $5: 95$ to $10: 90$ ) in $53 \%$ yield as colorless foam.

${ }^{1} \mathrm{H}$ NMR $\left(400 \mathrm{MHz}, \mathrm{CDCl}_{3}\right) \delta 9.66(\mathrm{~s}, 1 \mathrm{H}), 7.80-7.73(\mathrm{~m}, 2 \mathrm{H}), 7.68(\mathrm{~d}, J=8.5 \mathrm{~Hz}, 1 \mathrm{H})$, 7.62-7.61 (m, 2H), 7.49-7.46 (m, 2H), 7.27-7.09 (m, 13H), $7.01(\mathrm{~d}, J=7.6 \mathrm{~Hz}, 1 \mathrm{H}), 4.58$ (dd, $J=9.1,3.6 \mathrm{~Hz}, 1 \mathrm{H}), 4.31-4.26(\mathrm{~m}, 1 \mathrm{H}), 4.02(\mathrm{~d}, J=8.1 \mathrm{~Hz}, 1 \mathrm{H}), 3.90(\mathrm{~d}, J=14.0 \mathrm{~Hz}$, $1 \mathrm{H}), 3.85(\mathrm{~d}, J=14.3 \mathrm{~Hz}, 2 \mathrm{H}), 3.67(\mathrm{~d}, J=14.0 \mathrm{~Hz}, 2 \mathrm{H}), 3.13-3.07(\mathrm{~m}, 1 \mathrm{H}), 2.95-2.93(\mathrm{~m}, 1 \mathrm{H}), 2.22(\mathrm{~s}, 3 \mathrm{H})$, $1.21(\mathrm{~d}, J=6.1 \mathrm{~Hz}, 3 \mathrm{H})$.

${ }^{13} \mathrm{C}$ NMR $\left(100 \mathrm{MHz}, \mathrm{CDCl}_{3}\right) \delta 198.2,138.0,137.0,136.5,136.2,134.3,133.2,133.2,131.0,129.0,129.0$, 128.9 (2C), 128.0 (6C), 127.8, 127.8, 127.7, 127.6, 127.3, 126.8, 126.6, 126.4 (2C), 124.7, 78.4, 77.8, 72.3, $69.8,67.2,62.3,59.3,58.9,21.3,19.6$.

HRMS calculated for: $\left[\mathrm{C}_{39} \mathrm{H}_{38} \mathrm{~N}_{2} \mathrm{O}_{3}+\mathrm{H}\right]^{+}$583.2955; found: 583.2960 . The ee was determined by UPC ${ }^{2}$ using a Chiralpak IC-3 column [ $1 \% \mathrm{MeCN}$ (0.5 min), then gradient from 1\% to 40\% (10\%/min), $120 \mathrm{bar}, 40{ }^{\circ} \mathrm{C}$; flow rate $3.0 \mathrm{~mL} / \mathrm{min} ; \tau_{\text {major }}=4.49 \mathrm{~min}, \tau_{\text {minor }}=4.33 \mathrm{~min}(99 \%$ ee $) .[\alpha]_{D}^{25}=-58.8\left(\mathrm{c}=1.0, \mathrm{CH}_{2} \mathrm{Cl}_{2}\right)$.

\subsection{Organocatalytic synthesis of 7}

Tert-butyl (2R)-2-((3S,4R,5S)-2-benzyl-5-methyl-3-(o-tolyl)isoxazolidin-4-yl)-3-formylaziridine-1carboxylate, 7<smiles>Cc1ccccc1[C@@H]1[C@H](C)[C@H](C)ON1Cc1ccccc1</smiles>

as a colorless foam.

A glass vial equipped with a magnetic stirring bar was charged with compound $4 a$ (32.1 mg, $0.1 \mathrm{mmol}, 1.0$ equiv), catalyst 3b (14.9 mg, $0.025 \mathrm{mmol}, 0.25$ equiv), and $\mathrm{CH}_{2} \mathrm{Cl}_{2}(0.5 \mathrm{~mL})$. The mixture was stirred for $5 \mathrm{~min}$, then BocNHOTs $(34.5 \mathrm{mg}$, $0.12 \mathrm{mmol}, 1.2$ equiv) was added followed by NaOAc (24.6 mg, $0.3 \mathrm{mmol}, 3.0$ equiv). The reaction mixture was stirred for $24 \mathrm{~h}$ at room temperature. The product 7 was isolated by FC on silica (EtOAc/pentane 5:95 to 10:90) in 81\% yield

${ }^{1} \mathrm{H}$ NMR $\left(400 \mathrm{MHz}, \mathrm{CDCl}_{3}\right) \delta 9.92(\mathrm{~d}, J=4.1 \mathrm{~Hz}, 1 \mathrm{H}), 7.56(\mathrm{dd}, J=7.6,1.1 \mathrm{~Hz}, 1 \mathrm{H}), 7.30-7.11(\mathrm{~m}, 8 \mathrm{H}), 4.15(\mathrm{p}, J$ $=6.1 \mathrm{~Hz}, 1 \mathrm{H}), 4.08$ (bs, $1 \mathrm{H}), 3.93(\mathrm{~d}, J=14.2 \mathrm{~Hz}, 1 \mathrm{H}), 3.79(\mathrm{~d}, J=14.2 \mathrm{~Hz}, 1 \mathrm{H}), 3.03-2.99(\mathrm{~m}, 2 \mathrm{H}), 2.54-2.49$ $(\mathrm{m}, 1 \mathrm{H}), 2.33(\mathrm{~s}, 3 \mathrm{H}), 1.46(\mathrm{~d}, J=6.2 \mathrm{~Hz}, 3 \mathrm{H}), 1.45(\mathrm{~s}, 9 \mathrm{H})$.

${ }^{13} \mathrm{C}$ NMR $\left(100 \mathrm{MHz}, \mathrm{CDCl}_{3}\right) \delta 194.5,158.2,137.6,136.4,136.3,130.7,128.3(2 \mathrm{C}), 128.2,128.1(2 \mathrm{C}), 127.6$, $127.0,126.5,82.9,75.5,70.4,59.4,59.3,44.5,44.0,27.8(3 C), 20.7,19.7$.

HRMS calculated for: $\left[\mathrm{C}_{26} \mathrm{H}_{32} \mathrm{~N}_{2} \mathrm{O}_{4}+\mathrm{H}\right]^{+}$437.2435; found: 437.2445. The ee was determined by UPC ${ }^{2}$ using a Chiralpak IC-3 column [ $1 \% \mathrm{MeCN}$ (0.5 min), then gradient from $1 \%$ to $40 \%(10 \% / \mathrm{min}), 120$ bar, $40{ }^{\circ} \mathrm{C}$ ]; flow rate $3.0 \mathrm{~mL} / \mathrm{min} ; \tau_{\text {major }}=3.04 \mathrm{~min}, \tau_{\text {minor }}=2.90 \mathrm{~min}(94 \%$ ee $) .[\alpha]_{\mathrm{D}}^{25}=-57.1\left(\mathrm{c}=1.0, \mathrm{CH}_{2} \mathrm{Cl}_{2}\right)$. 


\section{The asymmetric triple 1,3-dipolar cycloaddition cascade of nitrones to $2,4,6$ - trienals}

\subsection{General procedure for the organocatalytic synthesis of $8 a, b$}

A glass vial equipped with a magnetic stirring bar was charged with catalyst $3 \mathrm{c}(31.3 \mathrm{mg}, 0.04 \mathrm{mmol}, 0.2$ equiv), aldehyde $1 \mathrm{~d}\left(24.4 \mathrm{mg}, 0.2 \mathrm{mmol}, 1.0\right.$ equiv), dry $\mathrm{CHCl}_{3}(0.9 \mathrm{~mL})$, and water $(10.8 \mu \mathrm{L}, 0.6 \mathrm{mmol}, 3.0$ equiv). The mixture was cooled to $-20{ }^{\circ} \mathrm{C}$ before a solution of $\mathrm{TfOH}$ in $\mathrm{CHCl}_{3}(100 \mu \mathrm{L}, 0.010 \mathrm{mmol}, 0.05$ equiv, $0.1 \mathrm{M})$ and nitrone $2\left(0.6 \mathrm{mmol}, 3.0\right.$ equiv) were added. The reaction was stirred at $4{ }^{\circ} \mathrm{C}$ until completion. $\mathrm{MeOH}(1.0 \mathrm{~mL})$ and $\mathrm{NaBH}_{4}(15.1 \mathrm{mg}, 0.4 \mathrm{mmol}, 2.0 \mathrm{eq})$ were added. The mixture was stirred at room temperature for $1 \mathrm{~h}$ before it was diluted with $\mathrm{CH}_{2} \mathrm{Cl}_{2}(1.0 \mathrm{~mL})$ and quenched with sat. aq. $\mathrm{NH}_{4} \mathrm{Cl}(1.0$ $\mathrm{mL})$. The aqueous phase was extracted with $\mathrm{CH}_{2} \mathrm{Cl}_{2}(3 \times 1.0 \mathrm{~mL})$. The combined organic phase was dried over $\mathrm{MgSO}_{4}$ and concentrated in vacuo. The diastereomeric ratio was determined by ${ }^{1} \mathrm{H} \mathrm{NMR}$ analysis of the crude reaction mixture by comparing the integrals of the peaks corresponding to the aldehyde protons of the diastereoisomeric products.

\subsection{Results and characterization}

((3'S,3"S,4'S,4"S,5'S,5"R)-3,3',3"-Tris(4-methoxyphenyl)-5-methyl-2,2',2"-triphenyl-[4,5':4',5"terisoxazolidin]-4"-yl)methanol, 8 a

Following the general procedure (reaction time $3 \mathrm{~d}$ ) the product $8 \mathrm{a}$ was isolated by FC on latrobeads (EtOAc/pentane $20: 80$ to $30: 70$ ) in $44 \%$ yield as a pale yellow foam. The diastereoisomers were separated by $\mathrm{FC}$ on latrobeads (EtOAc/ $\mathrm{CH}_{2} \mathrm{Cl}_{2}$ 1:99 to 3:97) for characterization.

((3S,3'S,3"S,4R,4'S,4"S,5S,5'S,5"R)-3,3',3"-Tris(4-methoxyphenyl)-5-methyl-2,2',2"'-triphenyl-[4,5':4',5"terisoxazolidin]-4"-yl)methanol, major-8a

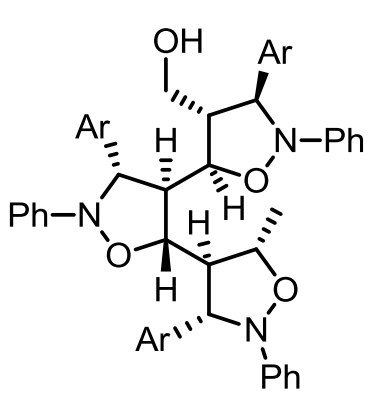

$\mathrm{Ar}=4-\mathrm{OMe}-\mathrm{C}_{6} \mathrm{H}_{4}$

${ }^{1} \mathrm{H} \mathrm{NMR}\left(400 \mathrm{MHz}, \mathrm{CDCl}_{3}\right) \delta 7.38(\mathrm{~d}, J=8.7 \mathrm{~Hz}, 2 \mathrm{H}), 7.25-7.12(\mathrm{~m}, 7 \mathrm{H}), 7.09(\mathrm{~d}, J=$ $8.7 \mathrm{~Hz}, 2 \mathrm{H}), 7.00-6.79(\mathrm{~m}, 16 \mathrm{H}), 5.02(\mathrm{~d}, J=3.8 \mathrm{~Hz}, 1 \mathrm{H}), 4.95(\mathrm{~d}, J=3.4 \mathrm{~Hz}, 1 \mathrm{H})$, 4.08-4.01 (m, 2H), $3.97(\mathrm{dd}, J=6.9,4.3 \mathrm{~Hz}, 1 \mathrm{H}), 3.89-3.82(\mathrm{~m}, 1 \mathrm{H}), 3.81(\mathrm{~s}, 3 \mathrm{H})$, $3.81(\mathrm{~s}, 3 \mathrm{H}), 3.80(\mathrm{~s}, 3 \mathrm{H}), 3.40-3.36(\mathrm{~m}, 1 \mathrm{H}), 3.30-3.24(\mathrm{~m}, 1 \mathrm{H}), 2.90-2.83(\mathrm{~m}, 2 \mathrm{H})$, 2.14-2.05 (m, 1H), $1.10(\mathrm{~d}, J=6.0 \mathrm{~Hz}, 3 \mathrm{H}), 0.87(\mathrm{t}, J=5.2 \mathrm{~Hz}, 1 \mathrm{H})$.

${ }^{13} \mathrm{C}$ NMR $\left(100 \mathrm{MHz}, \mathrm{CDCl}_{3}\right) \delta 159.0,158.7,158.5,151.6,150.4,150.1,135.3$, $134.0,132.5,128.9(2 \mathrm{C}), 128.8(2 \mathrm{C}), 128.7(2 \mathrm{C}), 127.9(2 \mathrm{C}), 127.8(2 \mathrm{C}), 127.6(2 \mathrm{C})$, $122.4,121.7,120.9,115.9(2 \mathrm{C}), 115.0(2 \mathrm{C}), 114.3(2 \mathrm{C}), 114.2(2 \mathrm{C}), 114.1(2 \mathrm{C})$, 114.0 (2C), 80.7, 79.0, 76.2, 72.3, 72.0, 71.1, 62.9, 62.3, 59.9, 59.6, 55.3, 55.3,

$55.2,18.3$ 
The ee was determined by UPC ${ }^{2}$ using a Chiralpak IA-3 column [1\% iPrOH ( $0.5 \mathrm{~min}$ ), then gradient from $1 \%$ to $40 \%(10 \% / \mathrm{min}), 120 \mathrm{bar}, 40{ }^{\circ} \mathrm{C}$; f flow rate $3.0 \mathrm{~mL} / \mathrm{min} ; \tau_{\text {major }}=5.36 \mathrm{~min}, \tau_{\text {minor }}=6.28 \mathrm{~min}\left(99 \%\right.$ ee). $[\alpha]^{25}$ $=-161.0\left(\mathrm{c}=1.0, \mathrm{CH}_{2} \mathrm{Cl}_{2}\right)$.

((3R,3'S,3"S,4S,4'S,4"S,5R,5'R,5"R)-3',3",5-Tris(4-methoxyphenyl)-3-methyl-2,2',2"-triphenyl-[4,5':4',5"terisoxazolidin]-4"-yl)methanol, minor-8a

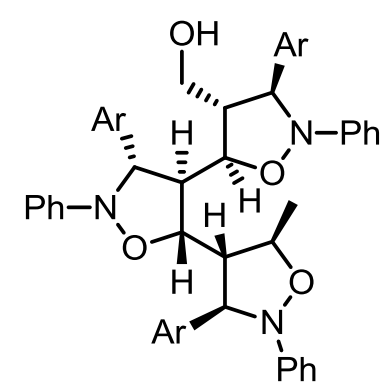

$\mathrm{Ar}=4-\mathrm{OMe}-\mathrm{C}_{6} \mathrm{H}_{4}$

${ }^{1} \mathrm{H}$ NMR $\left(400 \mathrm{MHz}, \mathrm{CDCl}_{3}\right) \delta$ 7.35-7.30 (m, 2H), 7.29-7.24 (m, 2H), 7.24-7.17 (m, $4 \mathrm{H}), 7.13(\mathrm{dd}, J=8.6,7.2 \mathrm{~Hz}, 2 \mathrm{H}), 7.01-6.85(\mathrm{~m}, 10 \mathrm{H}), 6.83(\mathrm{~d}, J=8.7 \mathrm{~Hz}, 2 \mathrm{H})$, 6.77-6.65 (m, $5 \mathrm{H}), 4.77(\mathrm{~d}, J=3.4 \mathrm{~Hz}, 1 \mathrm{H}), 4.50(\mathrm{p}, J=6.0 \mathrm{~Hz}, 1 \mathrm{H}), 4.31(\mathrm{~d}, J=3.6$ $\mathrm{Hz}, 1 \mathrm{H}), 4.17(\mathrm{~d}, J=4.8 \mathrm{~Hz}, 1 \mathrm{H}), 3.98(\mathrm{dd}, J=8.7,3.6 \mathrm{~Hz}, 1 \mathrm{H}), 3.83(\mathrm{~s}, 3 \mathrm{H}), 3.81-$ $3.74(\mathrm{~m}, 4 \mathrm{H}), 3.67(\mathrm{~s}, 3 \mathrm{H}), 3.42-3.37(\mathrm{~m}, 1 \mathrm{H}), 3.33-3.27(\mathrm{~m}, 1 \mathrm{H}), 3.21-3.17(\mathrm{~m}, 1 \mathrm{H})$, 2.98 (ddd, $J=9.6,6.1,3.6 \mathrm{~Hz}, 1 \mathrm{H}), 2.25-2.20(\mathrm{~m}, J=9.5,5.2 \mathrm{~Hz}, 1 \mathrm{H}), 1.37$ (d, $J=$ $6.0 \mathrm{~Hz}, 3 \mathrm{H}), 0.68(\mathrm{dd}, J=6.9,5.0 \mathrm{~Hz}, 1 \mathrm{H})$.

${ }^{13} \mathrm{C}$ NMR $\left(100 \mathrm{MHz}, \mathrm{CDCl}_{3}\right) \delta 159.0,158.8,158.5,151.9,150.2,150.1,134.1$, $133.4,133.2,128.9(2 \mathrm{C}), 128.8(2 \mathrm{C}), 128.7(2 \mathrm{C}), 128.0(2 \mathrm{C}), 127.4(2 \mathrm{C}), 127.1(2 \mathrm{C})$, 122.2, 121.7, 120.9, 115.6 (2C), 115.3 (2C), 114.5 (2C), 114.4 (2C), 114.2 (2C), $113.8(2 \mathrm{C}), 80.9,80.0,78.9$, $72.2,71.5,70.8,62.9,62.2,60.1,59.9,55.3(2 \mathrm{C}), 55.2,19.8$.

The ee was determined by UPC ${ }^{2}$ using a Chiralpak IA-3 column [1\% iPrOH (0.5 min), then gradient from $1 \%$ to $40 \%(10 \% / \mathrm{min}), 120 \mathrm{bar}, 40{ }^{\circ} \mathrm{C}$; f flow rate $3.0 \mathrm{~mL} / \mathrm{min}$; $\tau_{\text {major }}=7.09 \mathrm{~min}, \tau_{\text {minor }}=5.13 \mathrm{~min}(99 \%$ ee $) .[\alpha]^{25}$ $=-30.0\left(\mathrm{c}=0.1, \mathrm{CH}_{2} \mathrm{Cl}_{2}\right)$.

HRMS calculated for: $\left[\mathrm{C}_{50} \mathrm{H}_{51} \mathrm{~N}_{3} \mathrm{O}_{7}+\mathrm{H}\right]^{+}$806.3800; found: 806.3806 .

((3'S,3"S,4'S,4"S,5'S,5"R)-5-Methyl-3,3',3"--tri(naphthalen-2-yl)-2,2',2"-triphenyl-[4,5':4',5"terisoxazolidin]-4"-yl)methanol, $8 \mathrm{~b}$

Following the general procedure (reaction time $3 \mathrm{~d}$ ) the product $\mathbf{8} \mathbf{b}$ was isolated by FC on latrobeads (EtOAc/ $\mathrm{CH}_{2} \mathrm{Cl}_{2}$ 0:100 to $10: 90$ ) in $52 \%$ yield as a pale yellow foam. The diastereoisomers were separated by FC on latrobeads (EtOAc/pentane 5:95 to 10:90) for characterization.

((3S,3'S,3"S, $S R, 4$ 'S,4"S,5S,5'S,5"R)-5-Methyl-3,3',3"-tri(naphthalen-2-yl)-2,2',2"'-triphenyl-[4,5':4',5"terisoxazolidin]-4"-yl)methanol, major-8b

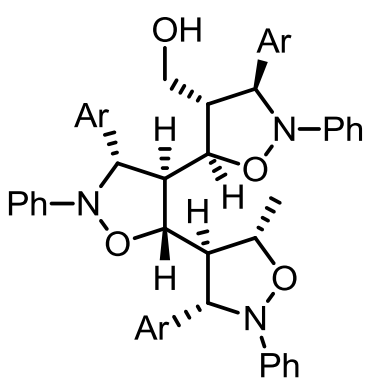

Ar = 2-naphthyl
${ }^{1} \mathrm{H}$ NMR $\left(400 \mathrm{MHz}, \mathrm{CDCl}_{3}\right) \delta 7.84-6.78(\mathrm{~m}, 36 \mathrm{H}), 5.20(\mathrm{~d}, J=3.4 \mathrm{~Hz}, 1 \mathrm{H}), 5.15(\mathrm{~d}, J=$ $4.1 \mathrm{~Hz}, 1 \mathrm{H}), 4.22(\mathrm{~d}, J=5.3 \mathrm{~Hz}, 1 \mathrm{H}), 4.06-4.02(\mathrm{~m}, 1 \mathrm{H}), 3.95-3.89(\mathrm{~m}, 2 \mathrm{H}), 3.36-$ $3.32(\mathrm{~m}, 1 \mathrm{H}), 3.27-3.19(\mathrm{~m}, 1 \mathrm{H}), 3.00(\mathrm{dt}, J=8.3,4.1 \mathrm{~Hz}, 1 \mathrm{H}), 2.88(\mathrm{td}, J=7.0,4.1$ $\mathrm{Hz}, 1 \mathrm{H}), 2.15(\mathrm{dq}, J=9.7,5.2 \mathrm{~Hz}, 1 \mathrm{H}), 0.81(\mathrm{~d}, J=6.0 \mathrm{~Hz}, 3 \mathrm{H})$. 
${ }^{13} \mathrm{C}$ NMR $\left(100 \mathrm{MHz}, \mathrm{CDCl}_{3}\right) \delta 151.5,150.4,149.9,140.4,139.5,137.9,133.4,133.2,133.2,132.8,132.7$, 132.6, 129.0 (3C), 129.0 (3C), 128.8 (3C), 128.6, 128.1, 128.0, 127.7, 127.7, 127.5, 126.4, 126.3, 126.1, $126.1,126.0,125.8,125.4,125.2,125.0,124.9,124.8,123.7,122.6,121.9,121.1,115.9$ (2C), 114.8 (2C), 114.1 (2C), 81.0, 79.1, 76.4, 72.6, 72.5, 71.7, 62.8, 62.5, 59.7, 59.6, 18.0.

The ee was determined by UPC ${ }^{2}$ using a Chiralpak IA-3 column [ $1 \% \mathrm{MeOH}(0.5 \mathrm{~min})$, then gradient from $1 \%$ to $40 \%(10 \% / \mathrm{min}), 120 \mathrm{bar}, 40{ }^{\circ} \mathrm{C}$; flow rate $3.0 \mathrm{~mL} / \mathrm{min} ; \tau_{\text {major }}=8.23 \mathrm{~min}, \tau_{\text {minor }}=9.84 \mathrm{~min}\left(99 \%\right.$ ee). $[\alpha]^{25}{ }_{\mathrm{D}}$ $=-158.1\left(\mathrm{c}=1.0, \mathrm{CH}_{2} \mathrm{Cl}_{2}\right)$.

((3R,3'S,3"S,4S,4'S,4"S,5R,5'S,5"R)-5-Methyl-3,3',3"-tri(naphthalen-2-yl)-2,2',2"'-triphenyl-[4,5':4',5"terisoxazolidin]-4"-yl)methanol, minor-8b

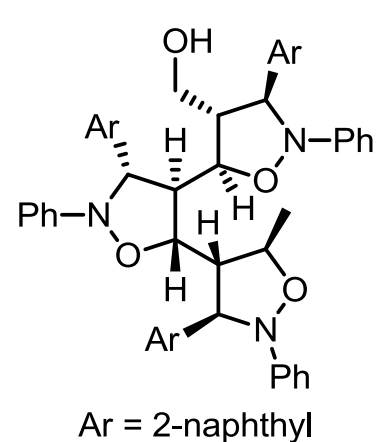

${ }^{1} \mathrm{H}$ NMR $\left(400 \mathrm{MHz}, \mathrm{CDCl}_{3}\right)$ 8 7.90-7.79 $(\mathrm{m}, 4 \mathrm{H}), 7.74(\mathrm{~d}, J=8.5 \mathrm{~Hz}, 1 \mathrm{H}), 7.65-7.27$ $(\mathrm{m}, 15 \mathrm{H}), 7.25-6.92(\mathrm{~m}, 12 \mathrm{H}), 6.90-6.84(\mathrm{~m}, 1 \mathrm{H}), 6.80(\mathrm{dd}, J=8.6,1.8 \mathrm{~Hz}, 1 \mathrm{H})$, 6.66-6.60 (m, 2H), $5.08(\mathrm{~d}, J=3.3 \mathrm{~Hz}, 1 \mathrm{H}), 4.66(\mathrm{p}, J=6.0 \mathrm{~Hz}, 1 \mathrm{H}), 4.41(\mathrm{~d}, J=3.2$ $\mathrm{Hz}, 1 \mathrm{H}), 4.37(\mathrm{~d}, J=4.8 \mathrm{~Hz}, 1 \mathrm{H}), 4.13(\mathrm{dd}, J=9.1,3.3 \mathrm{~Hz}, 1 \mathrm{H}), 3.89$ (dd, $J=9.6,2.2$ $\mathrm{Hz}, 1 \mathrm{H}), 3.51-3.39(\mathrm{~m}, 2 \mathrm{H}), 3.38-3.31(\mathrm{~m}, 1 \mathrm{H}), 3.19(\mathrm{ddd}, J=9.2,5.6,3.2 \mathrm{~Hz}, 1 \mathrm{H})$, 2.33-2.28 (m, $1 \mathrm{H}), 1.35(\mathrm{~d}, J=6.0 \mathrm{~Hz}, 3 \mathrm{H}), 0.62(\mathrm{dd}, J=7.2,5.1 \mathrm{~Hz}, 1 \mathrm{H})$.

${ }^{13} \mathrm{C}$ NMR $\left(100 \mathrm{MHz}, \mathrm{CDCl}_{3}\right) \delta 151.9,150.1,150.1,139.0,138.8,138.6,133.5$, $133.3,133.0,132.8,132.7,132.4,129.2,129.0$ (3C), 128.9 (2C), $128.8(2 \mathrm{C}), 128.3$, $128.1,127.9,127.7,127.6,127.6,127.3,126.5,126.3,126.1,126.0,125.8,125.6,125.5,124.9,124.8$, $124.4,123.9,123.7,122.5,121.8,121.0,115.7$ (2C), 115.2 (2C), 114.5 (2C), 81.2, 80.1, 78.9, 72.7, 72.1, 71.4, $62.9,61.8,60.2,60.1,20.0$.

The ee was determined by UPC ${ }^{2}$ using a Chiralpak IB-3 column [ $1 \% \mathrm{MeOH}(0.5 \mathrm{~min})$, then gradient from $1 \%$ to $40 \%(40 \% / \mathrm{min}), 120 \mathrm{bar}, 40{ }^{\circ} \mathrm{C}$; f flow rate $3.0 \mathrm{~mL} / \mathrm{min} ; \tau_{\text {major }}=5.12 \mathrm{~min}, \tau_{\text {minor }}=5.44 \mathrm{~min}(99 \%$ ee $) .[\alpha]^{25}$ $=-56.4\left(\mathrm{c}=0.5, \mathrm{CH}_{2} \mathrm{Cl}_{2}\right)$.

HRMS calculated for: $\left[\mathrm{C}_{59} \mathrm{H}_{51} \mathrm{~N}_{3} \mathrm{O}_{4}+\mathrm{H}\right]^{+}$866.3952; found: 866.3956 . 


\section{Reductive ring-opening of $5 \mathrm{c}$}<smiles>C[C@H]1ON(Cc2ccccc2)[C@H](c2ccc(Br)cc2)[C@@H]1[C@H]1C(C=O)ON(Cc2ccccc2)[C@H]1c1ccc(Br)cc1</smiles>

$5 c$
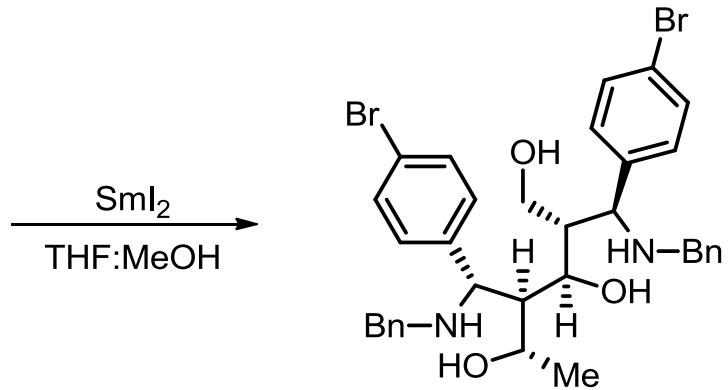

9

\section{$(2 S, 3 R, 4 R, 5 S)-2,4-B i s\left((S)\right.$-(benzylamino)(4-bromophenyl)methyl)hexane-1,3,5-triol, $9^{11}$}<smiles>C[C@H](O)[C@@H]([C@@H](CO)NCc1ccccc1)[C@@H](CO)[C@H](O)c1ccc(Br)cc1</smiles>

Compound $5 c$ ( $67.6 \mathrm{mg}, 0.1 \mathrm{mmol}, 1.0$ equiv) was dissolved in THF:MeOH (2.0 $\mathrm{mL}, 1: 1)$ under $\mathrm{Ar}$ atmosphere. $\mathrm{A} \mathrm{Sml}$-solution $(21.0 \mathrm{~mL}, 2.1 \mathrm{mmol}, 21.0$ equiv, $0.1 \mathrm{M}$ in THF) was added dropwise at room temperature and the reaction mixture was stirred for $4.5 \mathrm{~h}$. The flask was opened to the air and left to stir until the blue solution turned yellow. A small amount of water was added and the mixture diluted with $\mathrm{CH}_{2} \mathrm{Cl}_{2}(10 \mathrm{~mL})$ and stirred until it turned into a white slurry which was filtered over celite. The filter cake was washed with $\mathrm{CH}_{2} \mathrm{Cl}_{2}$ and the resulting mixture was washed with aq. sat. $\mathrm{NaHCO}_{3}(20 \mathrm{~mL})$. The aqueous phase was extracted with $\mathrm{CH}_{2} \mathrm{Cl}_{2}(3 \times 10 \mathrm{~mL})$ and the combined organic layer was washed with water $(20 \mathrm{~mL})$, brine $(20$ $\mathrm{mL}$ ), dried over $\mathrm{Na}_{2} \mathrm{SO}_{4}$, and concentrated in vacuo. The product 9 was isolated by $\mathrm{FC}$ on silica (EtOAc/pentane 50:50 to 100:0) in 54\% yield as a pale yellow solid.

${ }^{1} \mathrm{H}$ NMR $\left(400 \mathrm{MHz}, \mathrm{CDCl}_{3}\right)$ 8 7.47-7.40 (m, 2H), $7.36(\mathrm{~d}, J=8.4 \mathrm{~Hz}, 2 \mathrm{H}), 7.30-7.22(\mathrm{~m}, 5 \mathrm{H}), 7.19-7.10(\mathrm{~m}, 6 \mathrm{H})$, 7.10-7.04 (m, 3H), 4.23-4.14 (m, 1H), $4.12(\mathrm{~d}, J=4.7 \mathrm{~Hz}, 1 \mathrm{H}), 4.04(\mathrm{~d}, J=7.2 \mathrm{~Hz}, 1 \mathrm{H}), 3.71-3.62(\mathrm{~m}, 2 \mathrm{H}), 3.46-$ $3.35(\mathrm{~m}, 4 \mathrm{H}), 3.27(\mathrm{dd}, J=11.1,5.5 \mathrm{~Hz}, 1 \mathrm{H}), 3.09-3.03(\mathrm{~m}, 1 \mathrm{H}), 1.69-1.64(\mathrm{~m}, 1 \mathrm{H}), 0.96(\mathrm{~d}, J=6.4 \mathrm{~Hz}, 3 \mathrm{H})$.

${ }^{13} \mathrm{C} \mathrm{NMR}\left(100 \mathrm{MHz}, \mathrm{CDCl}_{3}\right) \delta 141.5,139.0,138.9,138.5,131.8(2 \mathrm{C}), 131.5$ (2C), $129.9(2 \mathrm{C}), 129.3(2 \mathrm{C}), 128.8$ (2C), 128.6 (2C), 128.4 (2C), 128.3 (2C), 127.5, 127.3, 121.4, 120.7 73.5, 70.6, 63.3, 60.7, 60.2, 51.5, 51.4, $50.9,46.8,22.4$.

HRMS calculated for: $\left[\mathrm{C}_{34} \mathrm{H}_{39} \mathrm{Br}_{2} \mathrm{~N}_{2} \mathrm{O} 3+\mathrm{H}\right]^{+}$681.1322, 683.1301, 685.1281; found: 681.1326, 683.1308, 685.1295. The ee was determined by $\mathrm{UPC}^{2}$ using a Chiralpak AMY-1 column [gradient from $3 \%$ (EtOH/iPrOH $1: 1,0.2 \%$ TFA) to $60 \%(38 \% / \mathrm{min}), 220 \mathrm{bar}, 10{ }^{\circ} \mathrm{C}$; flow rate $1.2 \mathrm{~mL} / \mathrm{min} ; \tau_{\text {major }}=1.39 \mathrm{~min}, \tau_{\operatorname{minor}}=1.17 \mathrm{~min}$ $\left(99 \%\right.$ ee). $[\alpha]_{D}^{25}=-20.4\left(c=1.0, \mathrm{CH}_{2} \mathrm{Cl}_{2}\right)$. 


\section{Determination of the absolute configuration}

X-Ray structure of $\left(\left(3 S, 3{ }^{\prime} S, 4 R, 4^{\prime} S, 5 S, 5^{\prime} R\right)-2,2^{\prime}\right.$-dibenzyl-3,3'-bis(4-bromophenyl)-5-methyl-[4,5'biisoxazolidin]-4'-yl)methanol $\left(\mathbf{5} \mathbf{c}^{\prime}\right)$. The single crystal was obtained by slow evaporation of a solution of $\mathbf{5} \mathbf{c}^{\prime}$ in $\mathrm{MeOH}$.
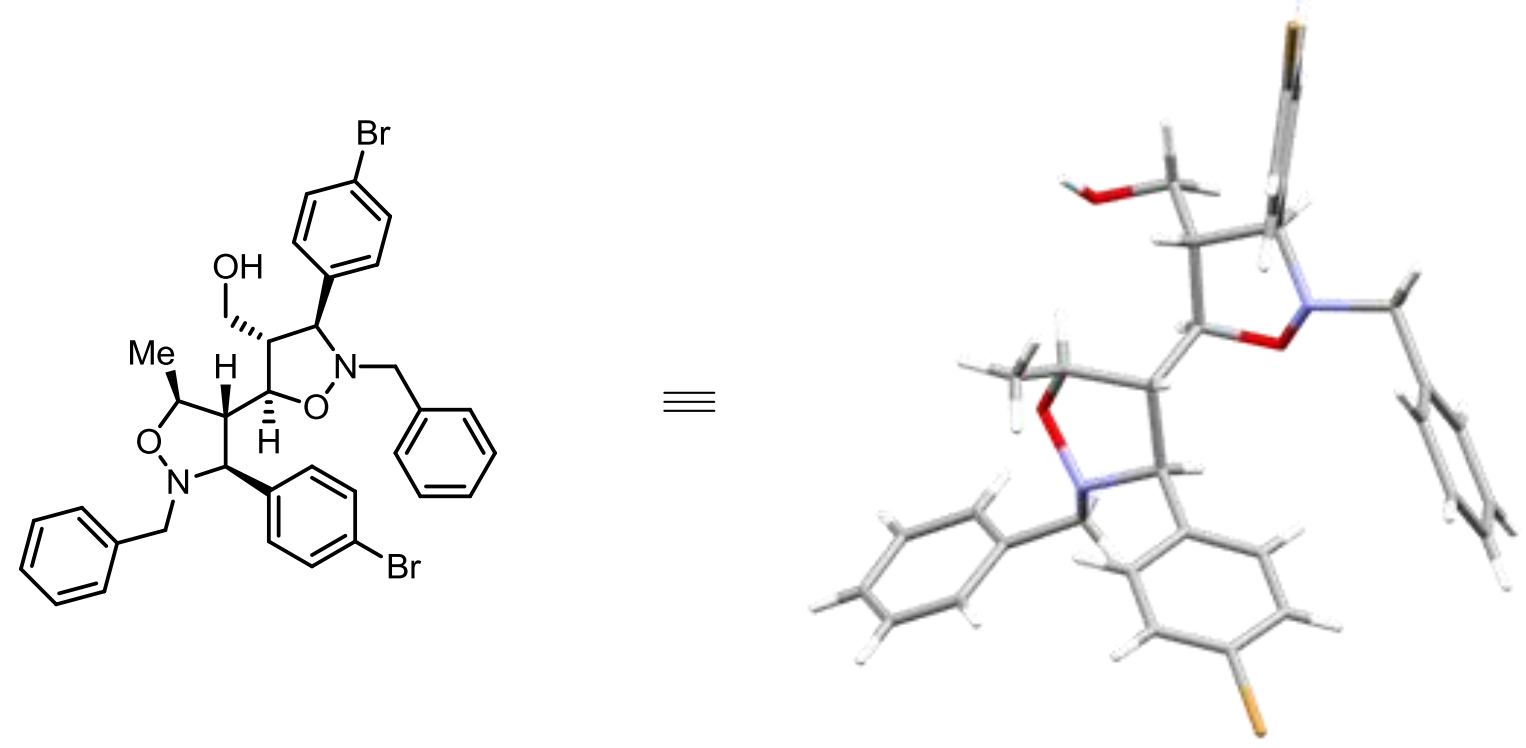

Crystal data for [5c']: $\mathrm{C}_{34} \mathrm{H}_{34} \mathrm{Br}_{2} \mathrm{~N}_{2} \mathrm{O}_{3}, \mathrm{M}=678.45$, monoclinic, space group I 121 (no. 11), $\mathrm{a}=17.3093(4)$ $\AA, b=6.24868(15) \AA, c=29.0192(8) \AA, \beta=102.724(3)^{\circ}$, Flack parameter $=-0.007, V=3061.65(13) \AA^{3}, T=$ $100 \mathrm{~K}, \mathrm{Z}=4, \mathrm{~d}_{\mathrm{c}}=1.474 \mathrm{~g} \mathrm{~cm}^{-3}, \mu($ Mo $\mathrm{K} \alpha, \lambda=0.71073 \AA$ ) $)=2.685 \mathrm{~mm}^{-1}, 33393$ reflections collected, 7311 unique $\left[R_{\text {int }}=0.0434\right]$, which were used in all calculations. Refinement on $F^{2}$, final $R(F)=0.0545, R_{w}(F 2)=$ 0.1217. CCDC number 1471261. 


\section{References}

${ }^{1}$ Poulsen, P. H.; Feu, K. S.; Paz, B. M.; Jensen, F.; Jørgensen, K. A. Angew. Chem. Int. Ed. 2015, 54, 8203.

${ }^{2}$ Zelenin, K. N.; Bezhan, I. P.; Ershov, A. Y. Chem. Heterocycl. Compd. 1988, 688.

${ }^{3}$ Yavuz, S.; Ozkan, H.; Colak, N.; Yildirir, Y. Molecules 2011, 16, 6677.

${ }^{4}$ Evans, D. A.; Song, H.-J.; Fandrick, K. R. Org. Lett. 2006, 8, 3351.

${ }^{5}$ Cicchi, S.; Corsi, M.; Goti, A. J. Org. Chem. 1999, 64, 7243.

${ }^{6}$ Pagoti, S.; Dutta, D.; Dash, J. Adv. Synth. Catal. 2013, 355, 3532.

${ }^{7}$ Lu, C.; Dubrovskiy, A. V.; Larock, R. C. J. Org. Chem. 2012, 77, 2279.

${ }^{8}$ Soldaini, G.; Cardona, F.; Goti, A. Org. Lett. 2007, 9, 473.

${ }^{9}$ Parisotto, S.; Boggio, P.; Prandi, C.; Venturello, P.; Deagostino, A. Tetrahedron Lett. 2015, 56, 5791.

${ }^{10}$ Prakash, G. K. S.; Zhang, Z.; Wang, F.; Rahm, M.; Ni, C.; Iuliucci, M.; Haiges, R.; Olah, G. A. Chem. Eur. J. 2014, 20, 831.

${ }^{11}$ Lykke, L.; Carlsen, B. D.; Scheibler, R. R.; Jørgensen, K. A. J. Am. Chem. Soc., 2014, $136,11296$. 

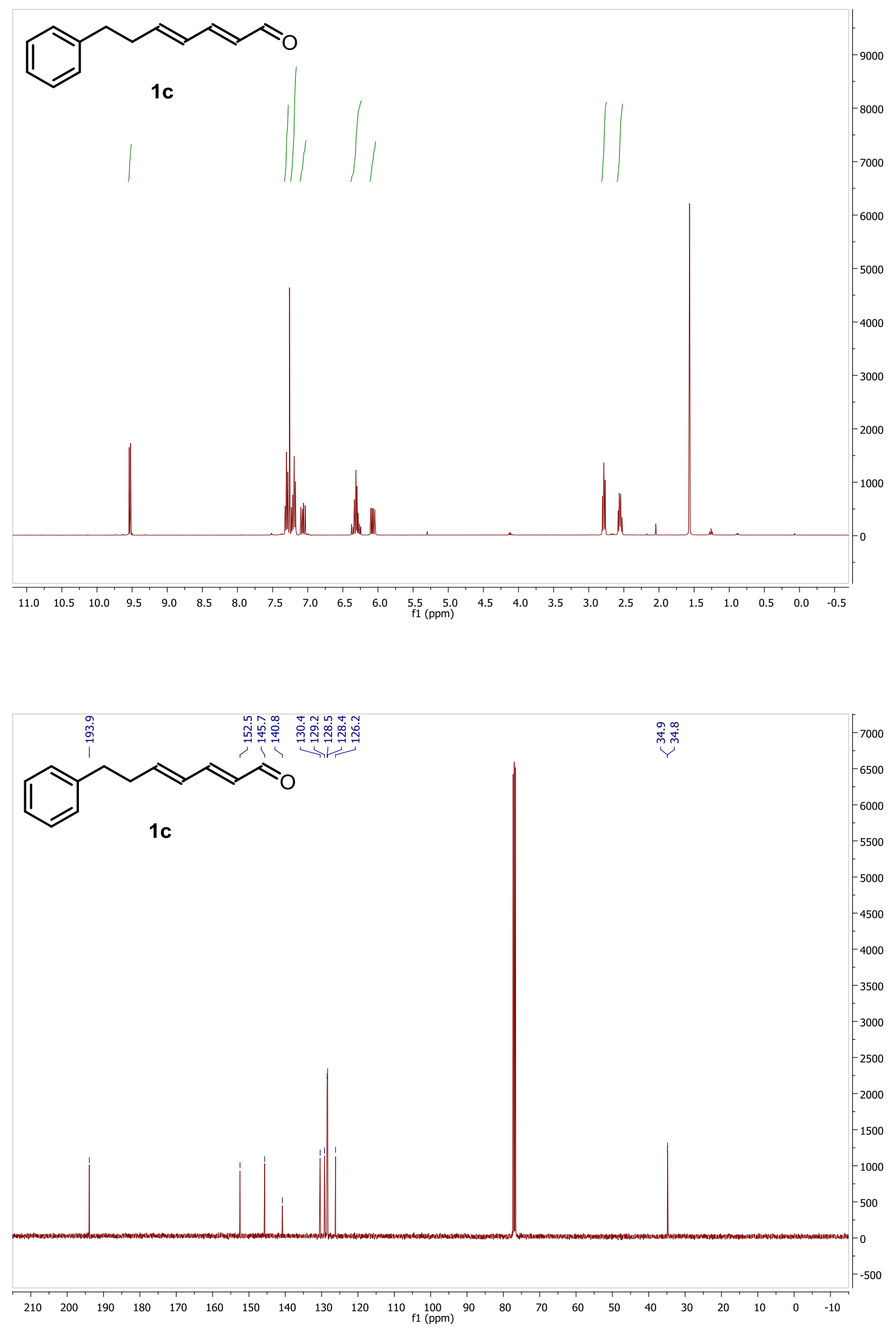

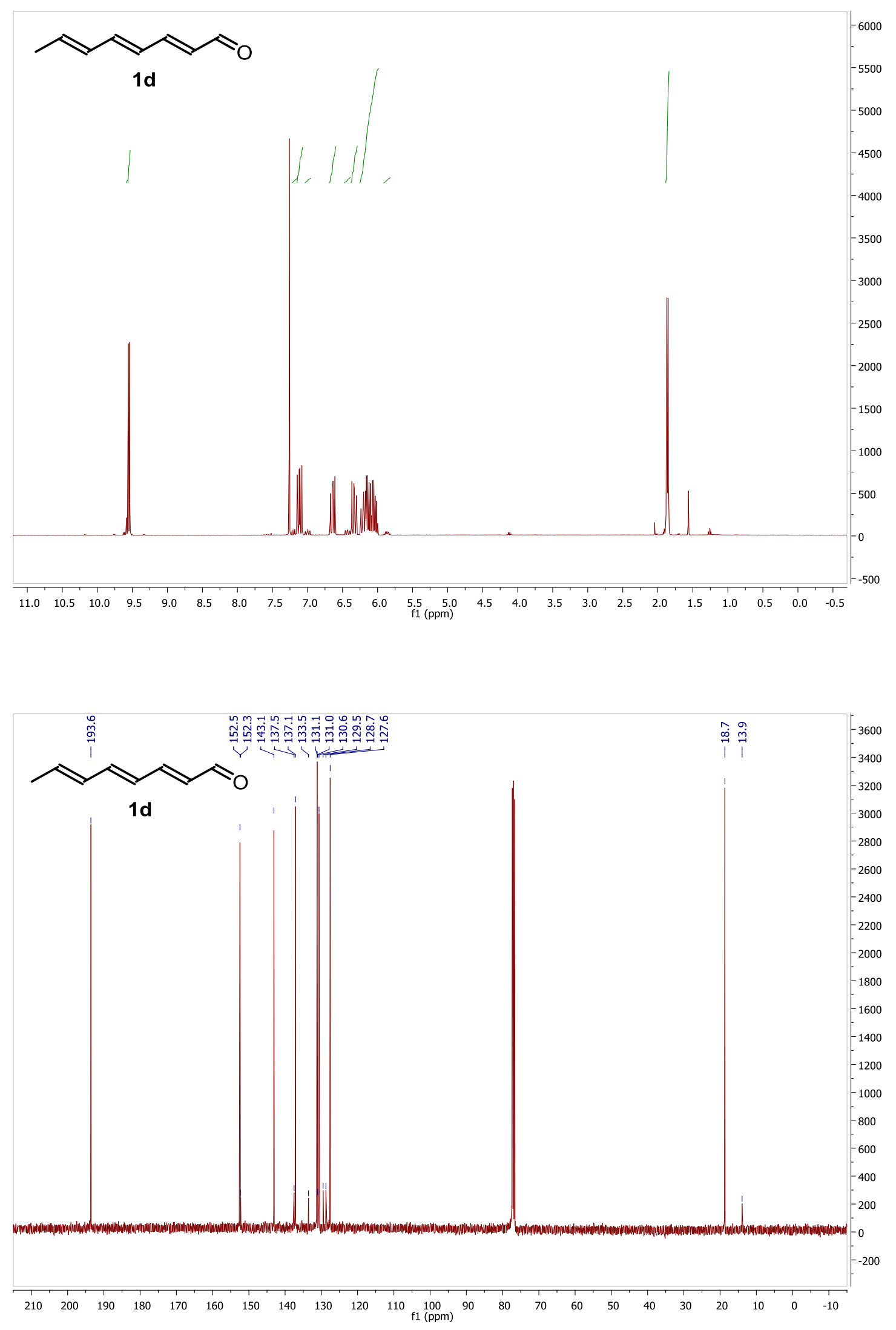

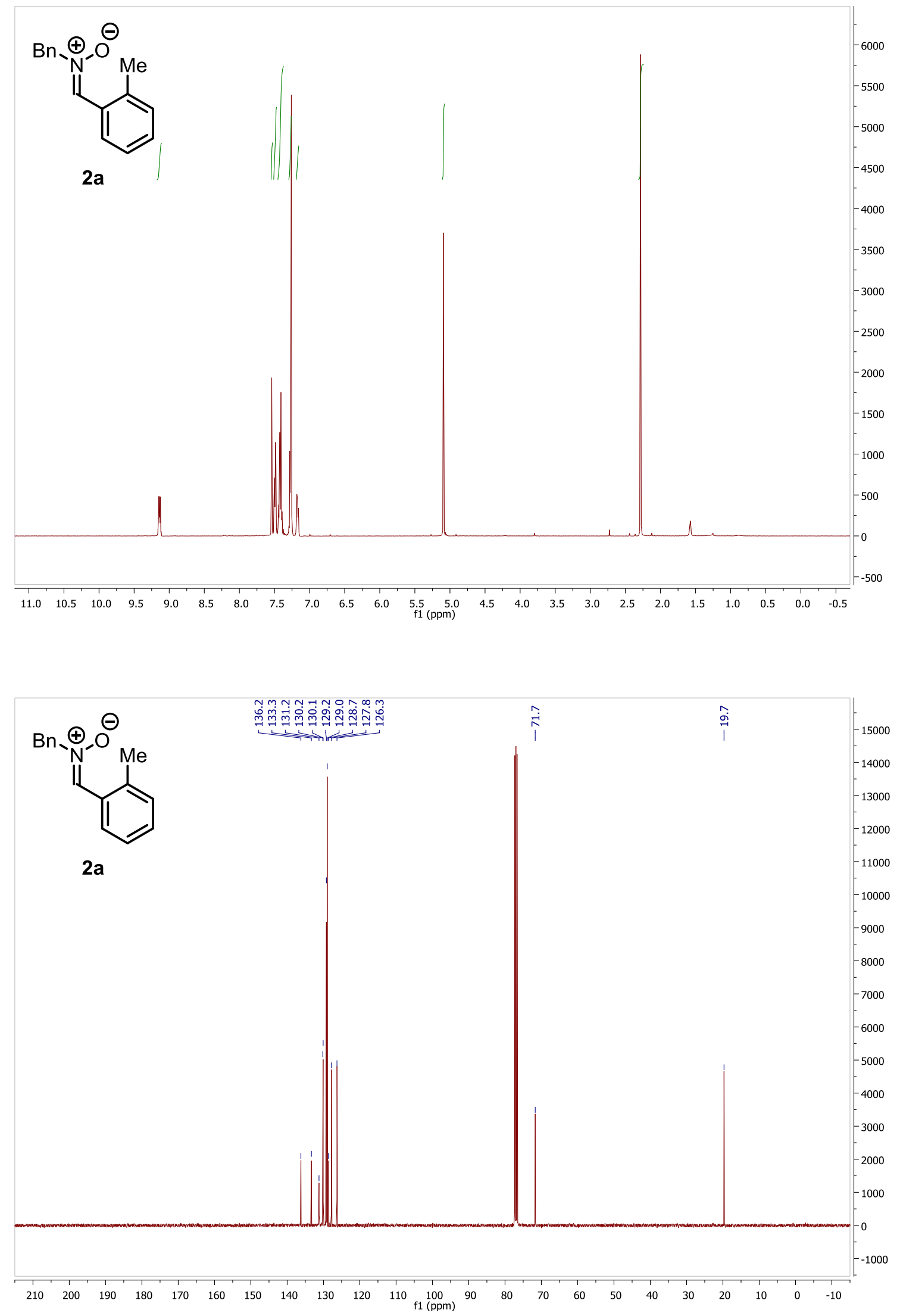

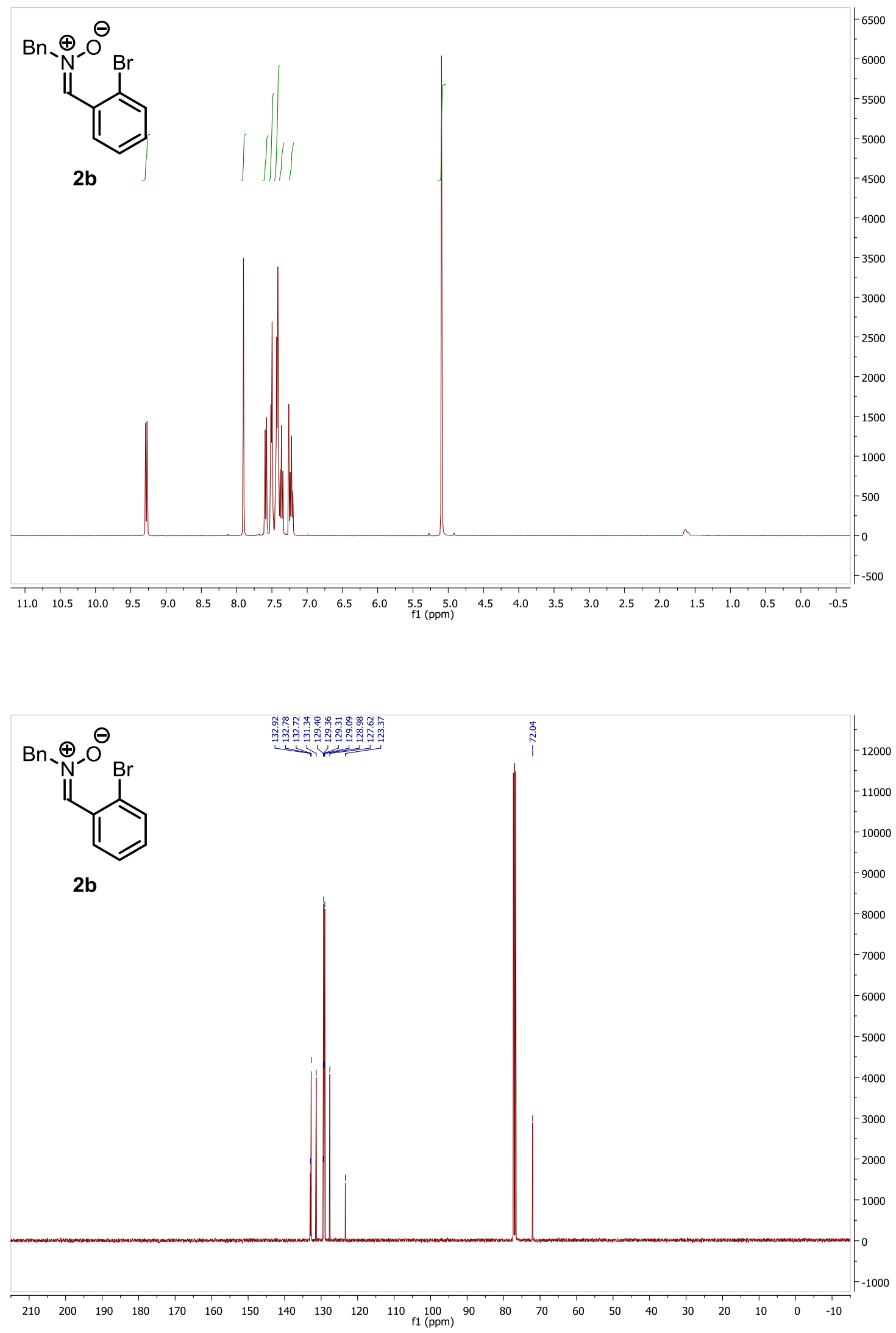

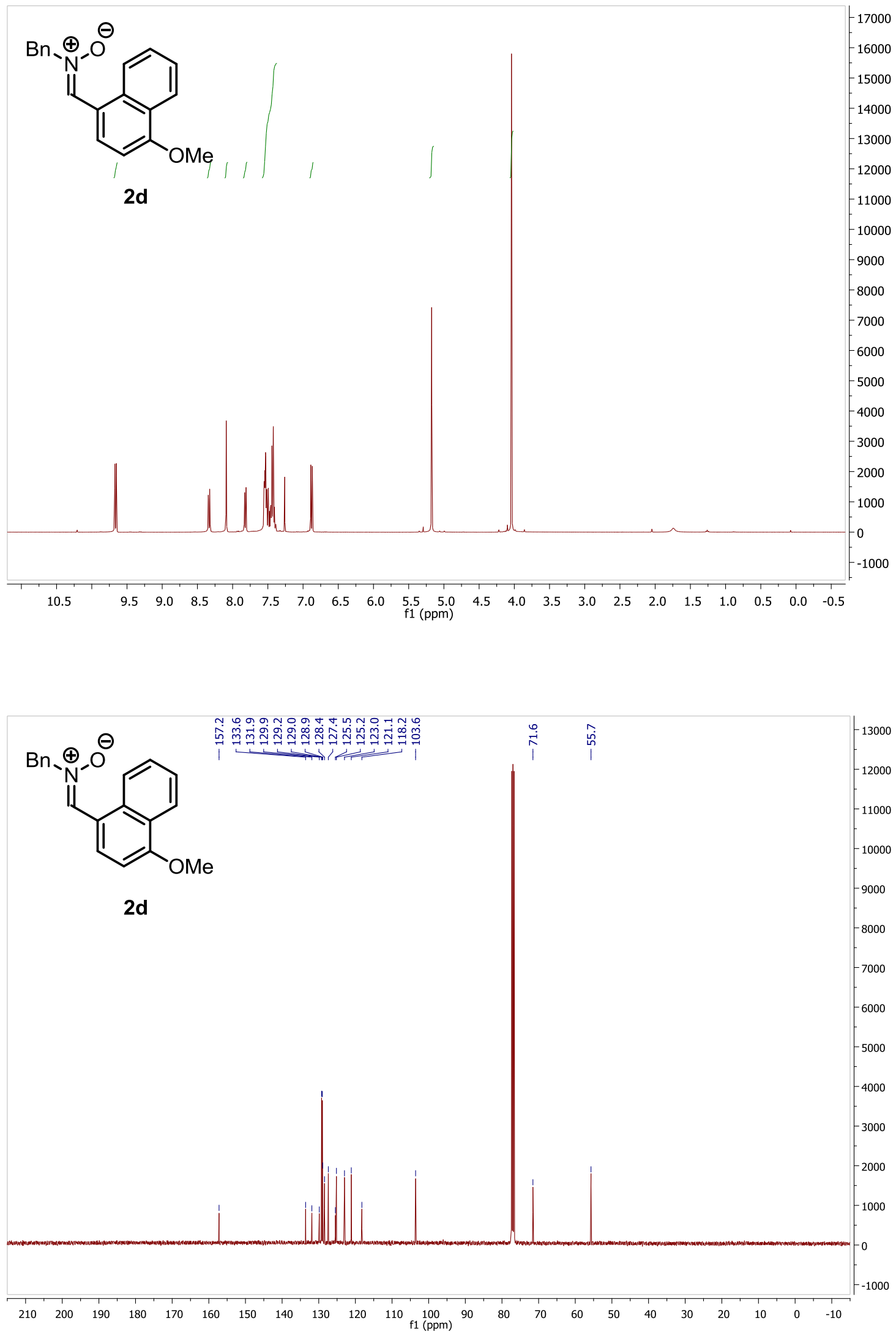

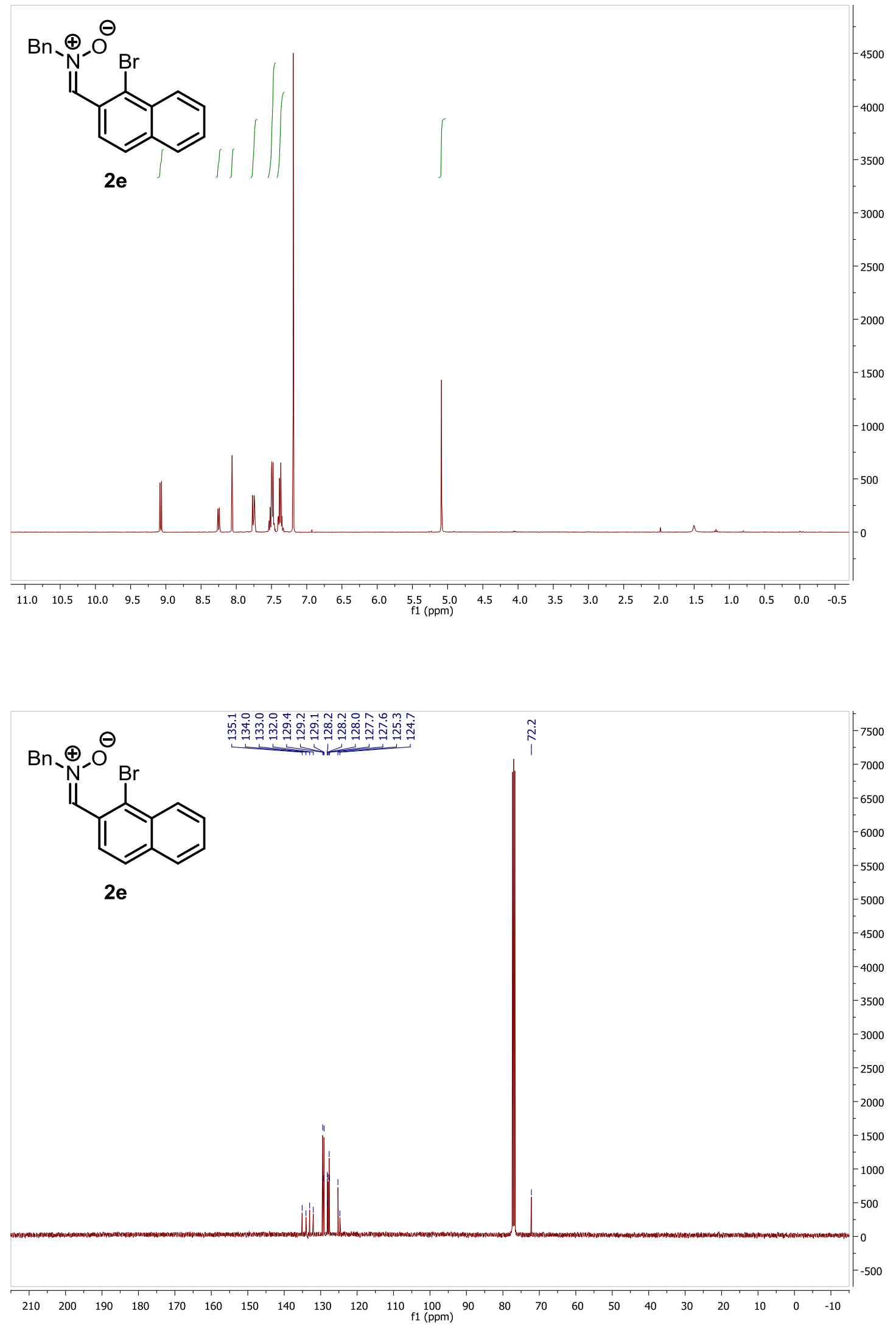

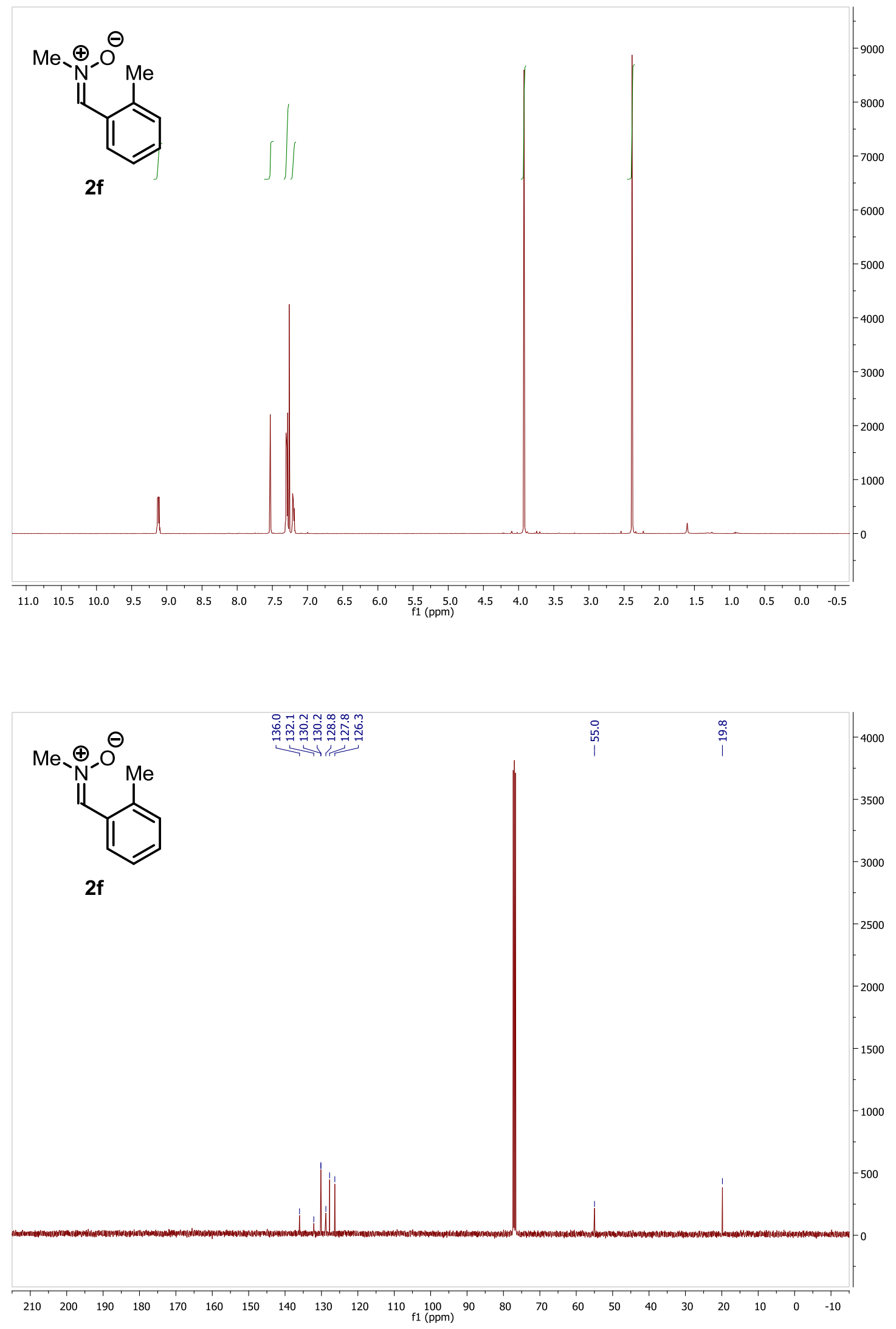

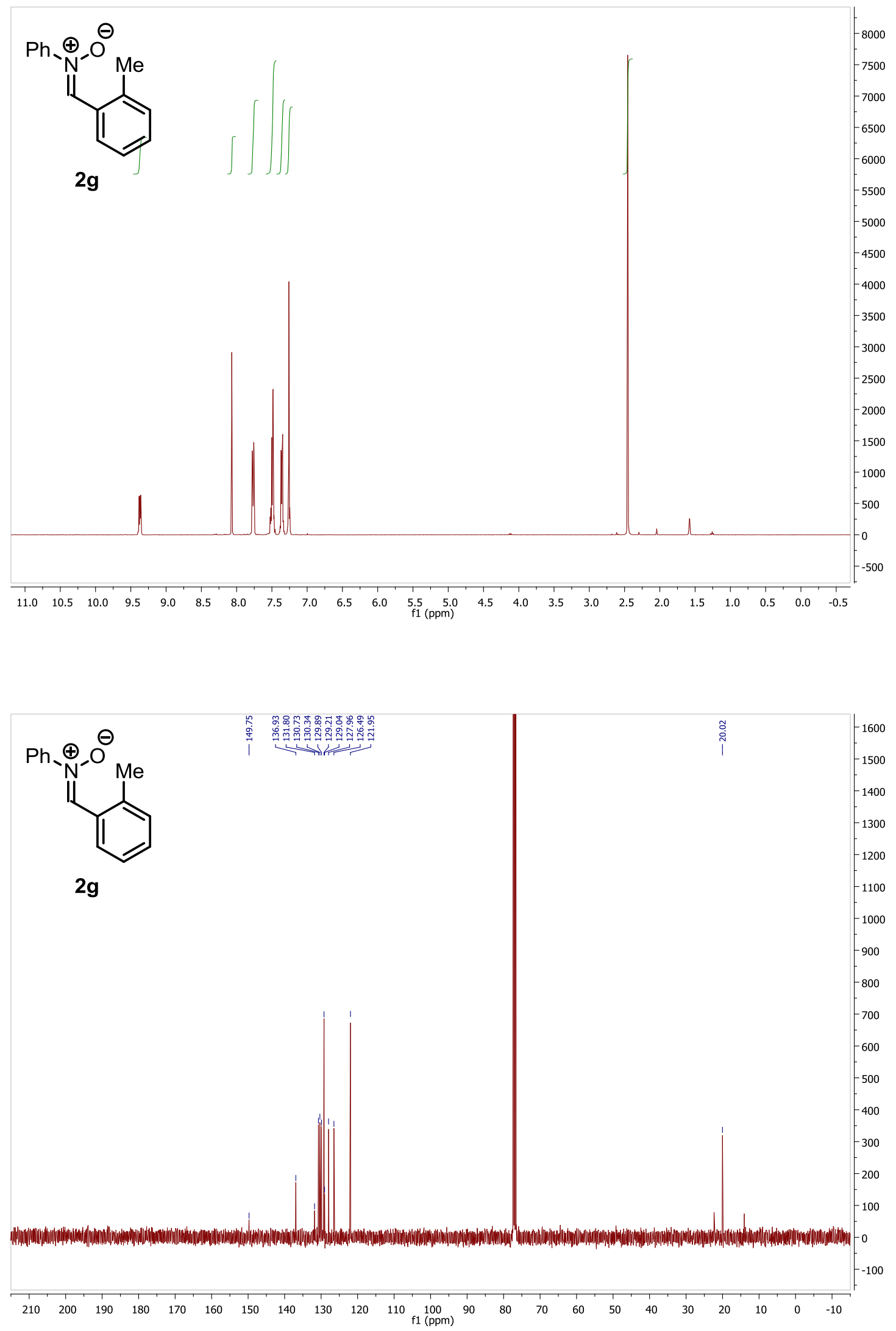

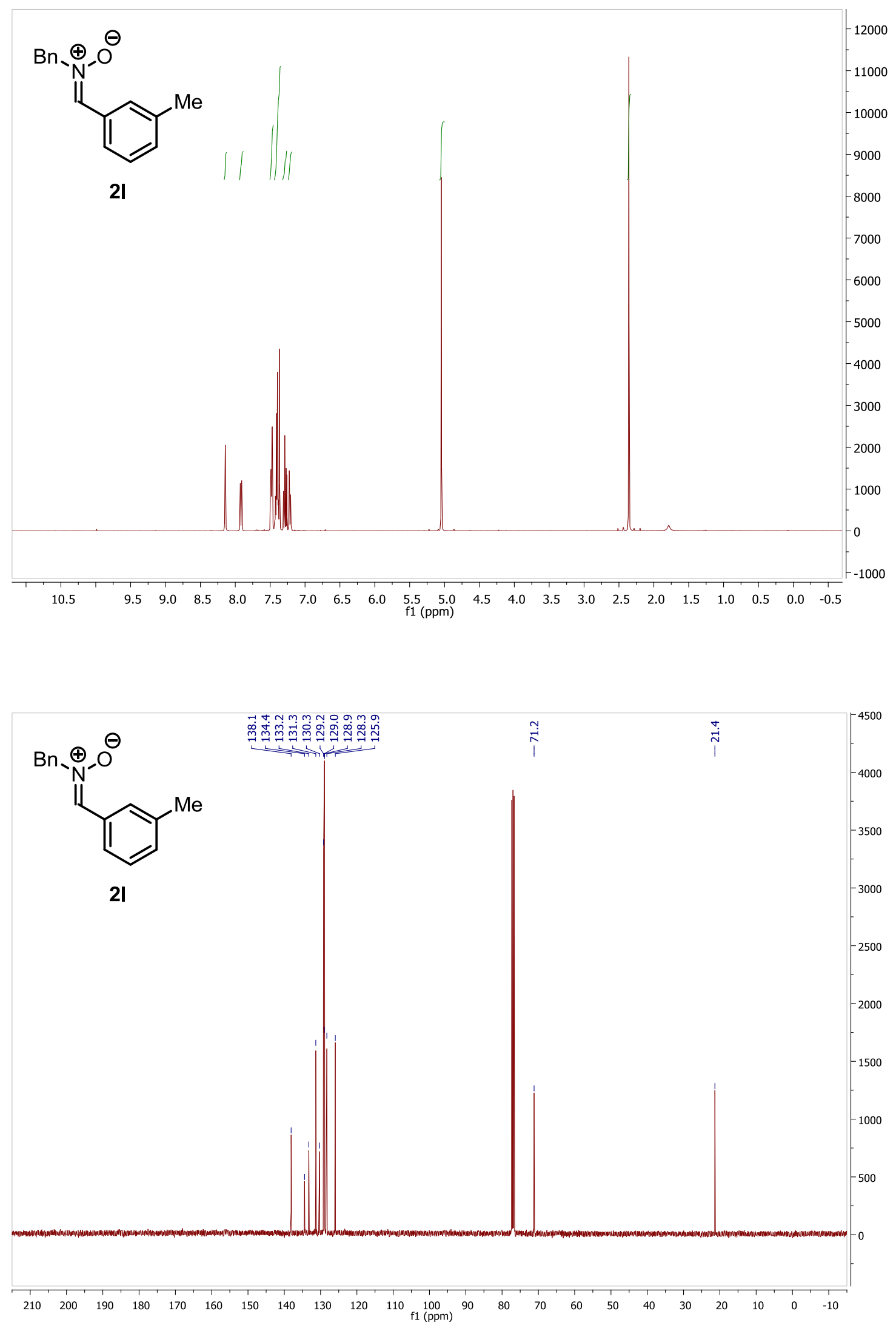

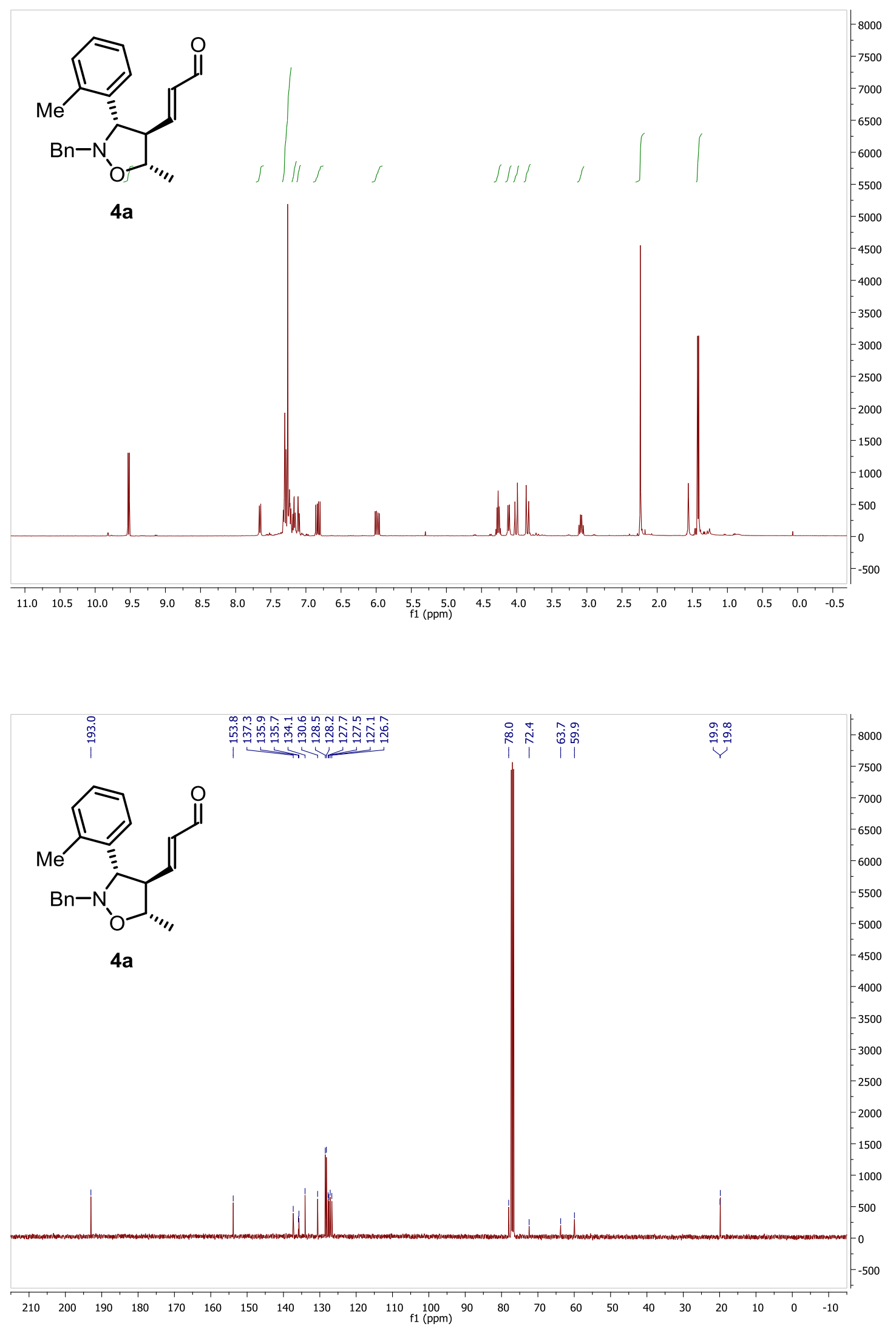

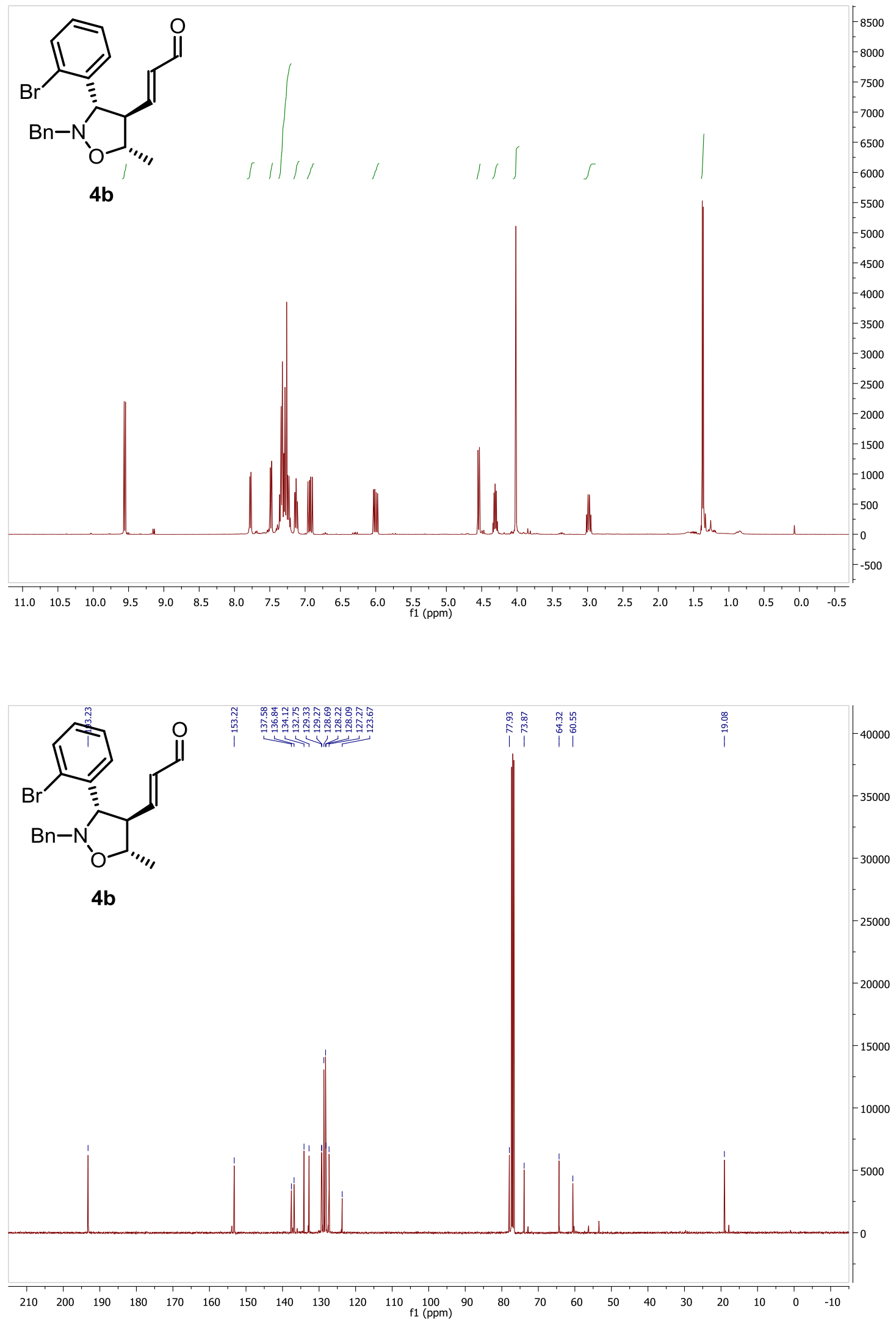

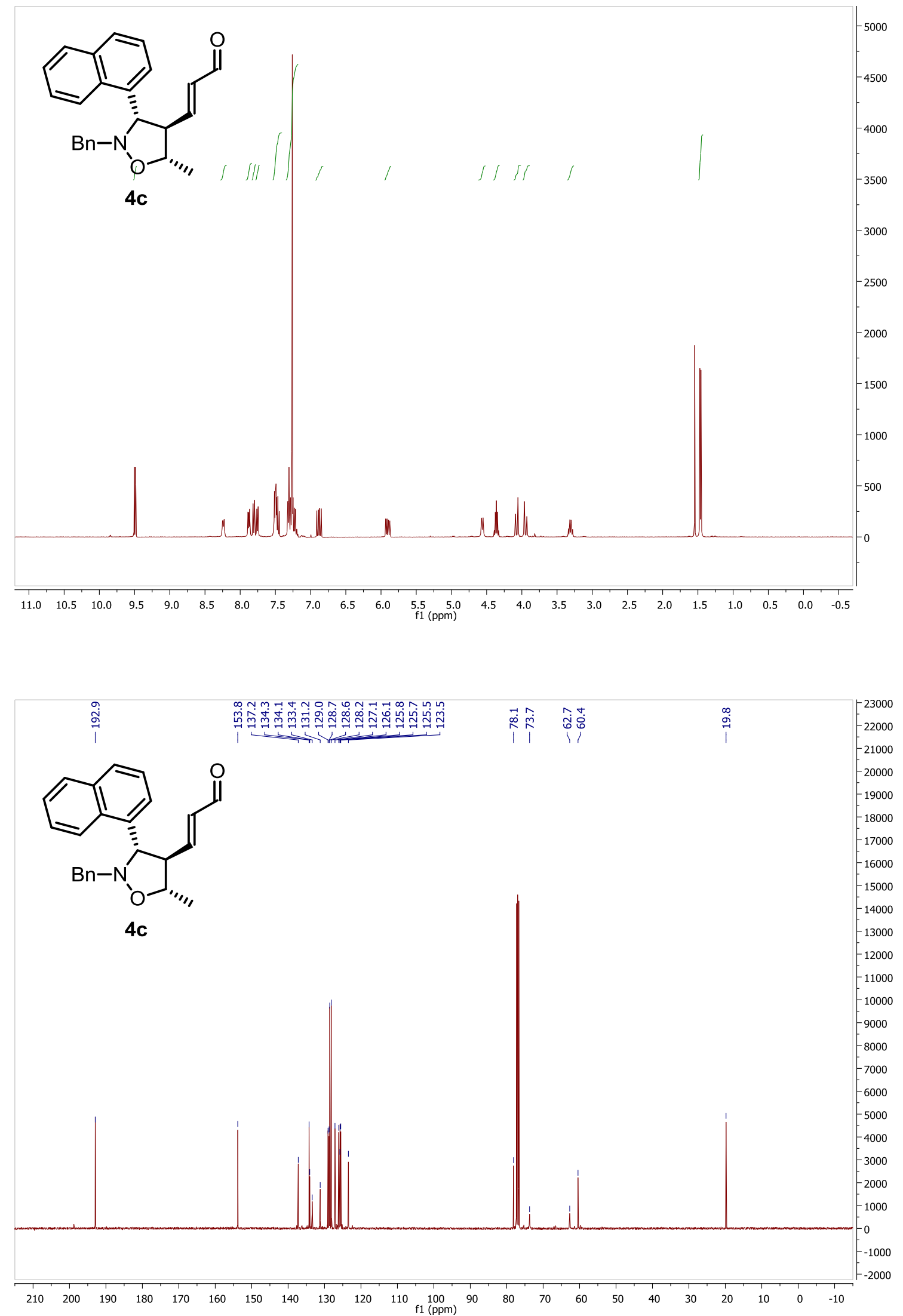

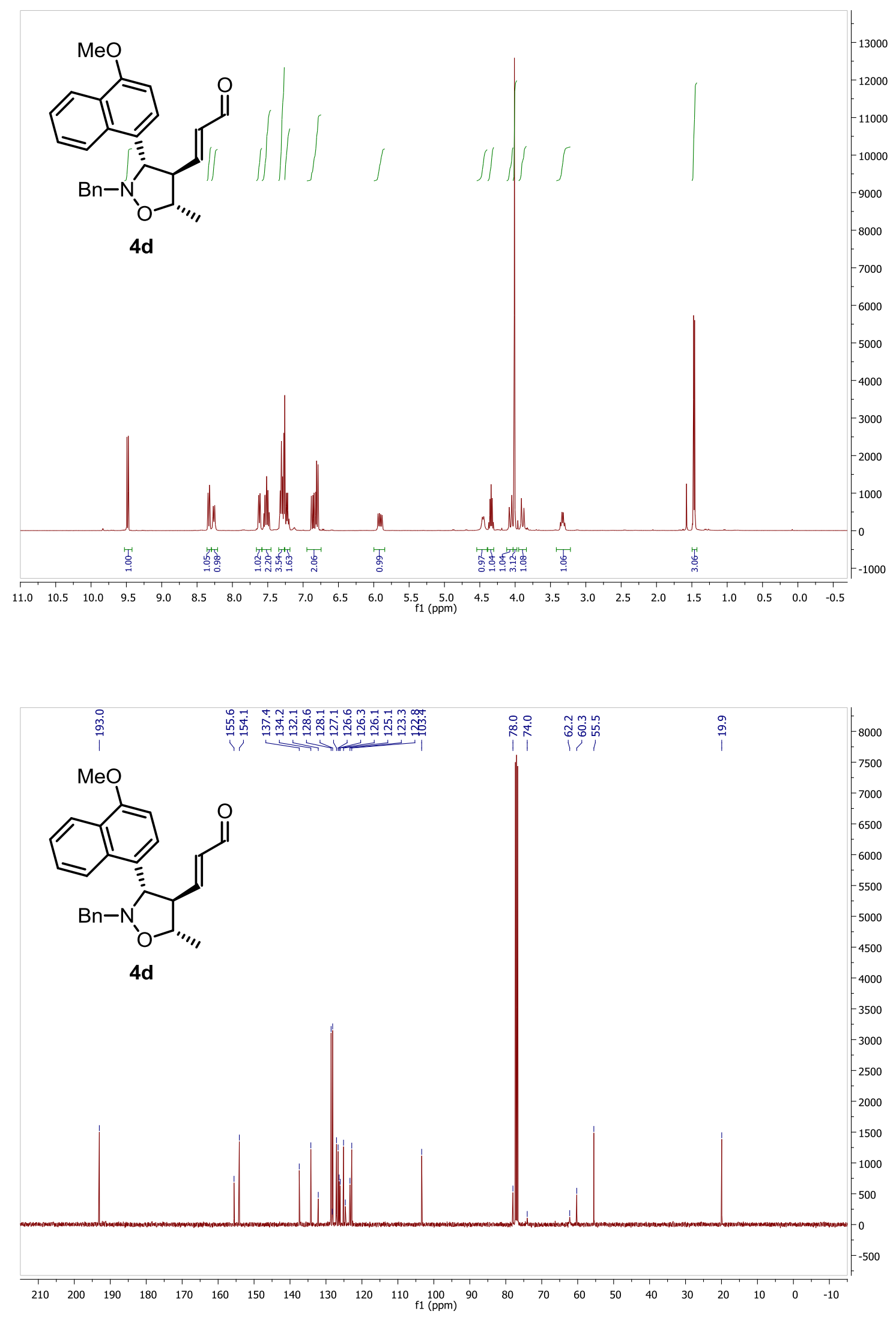

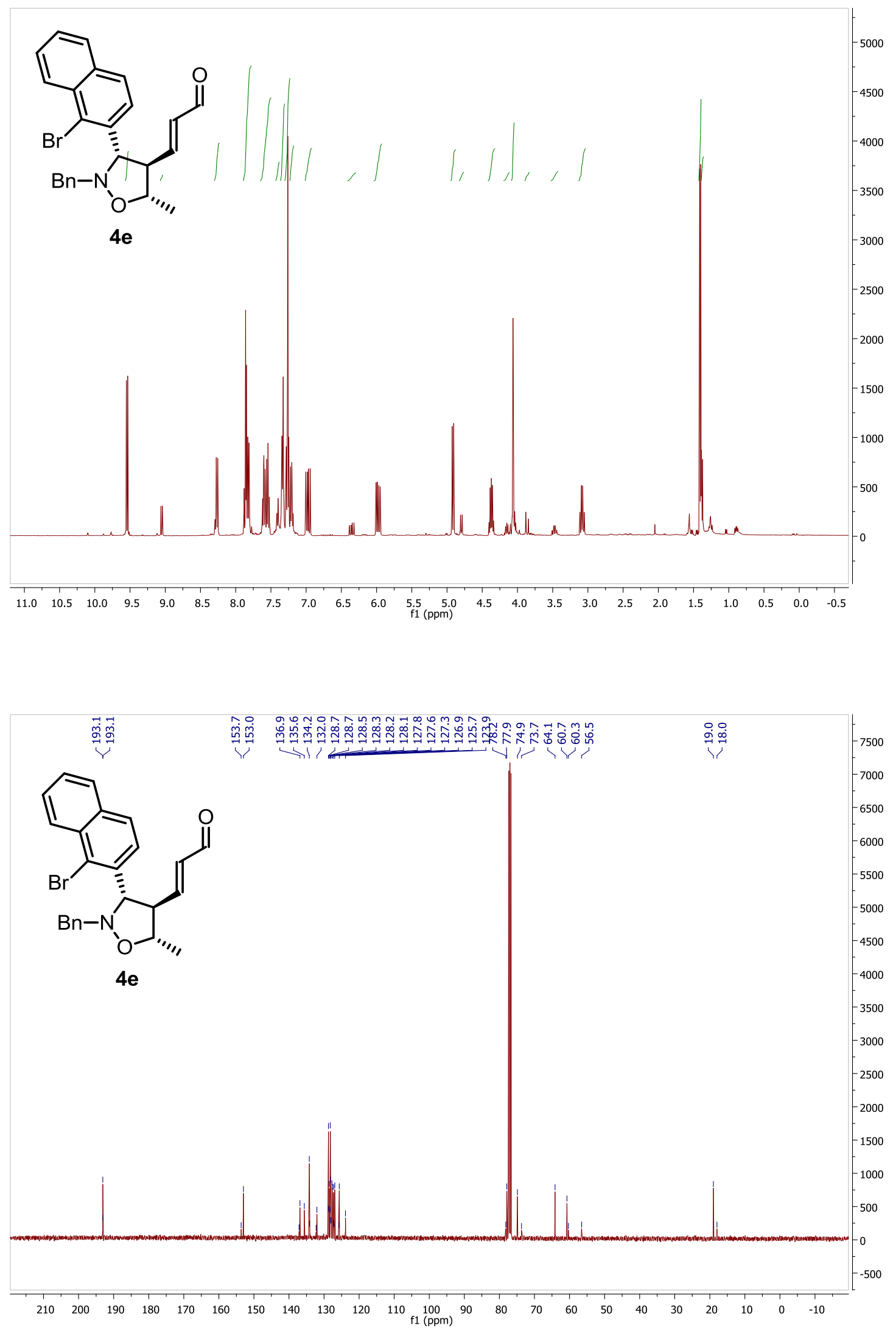

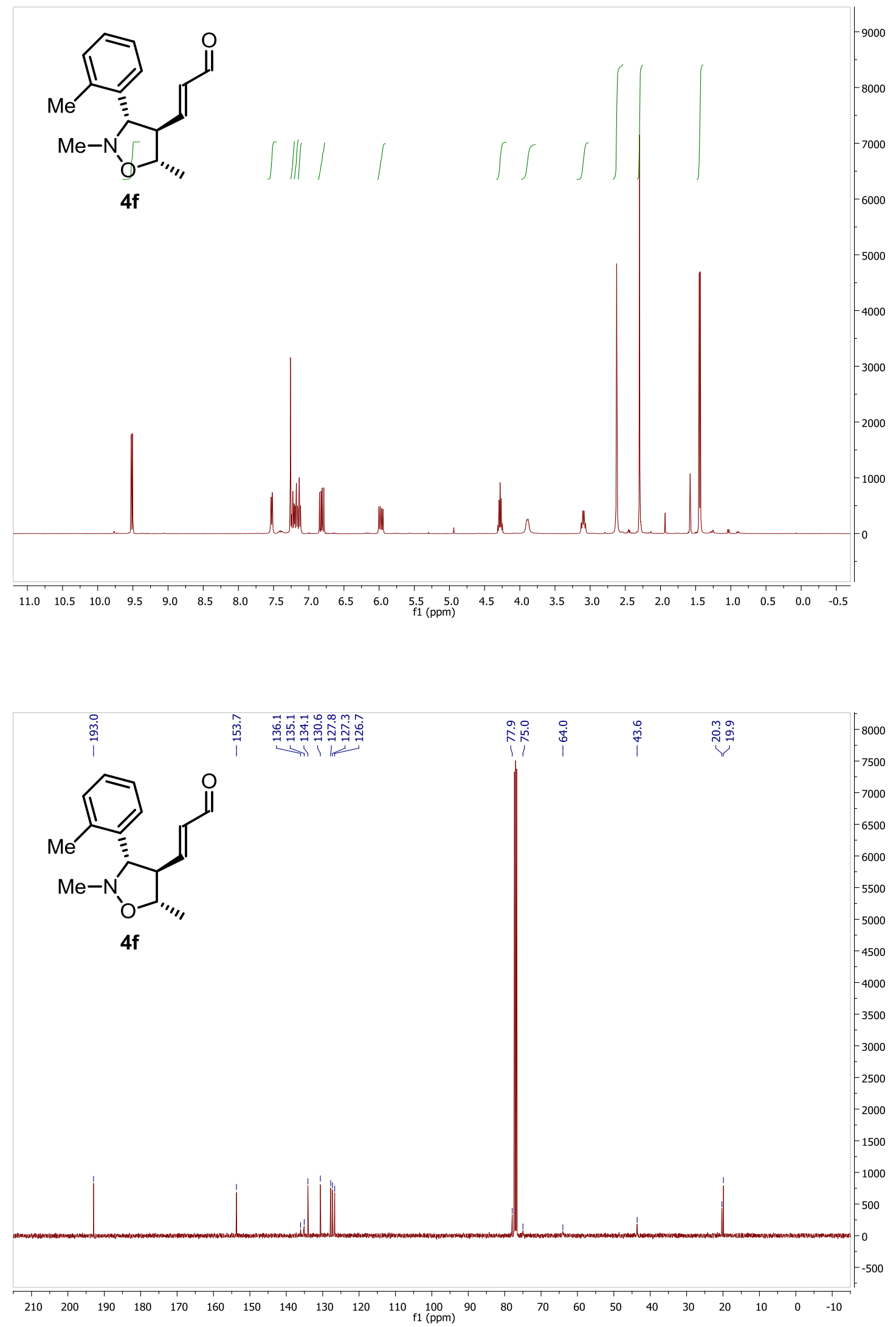

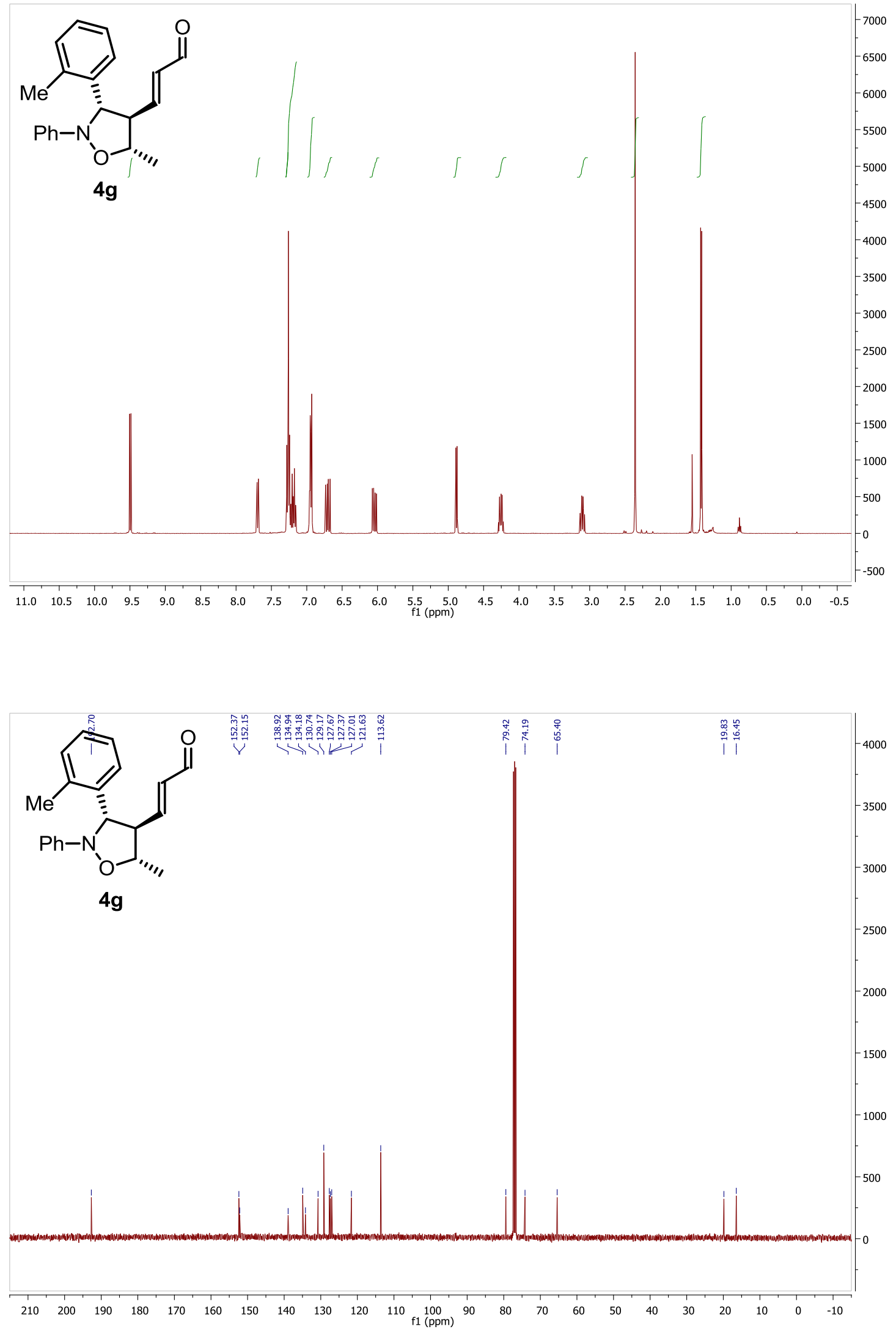

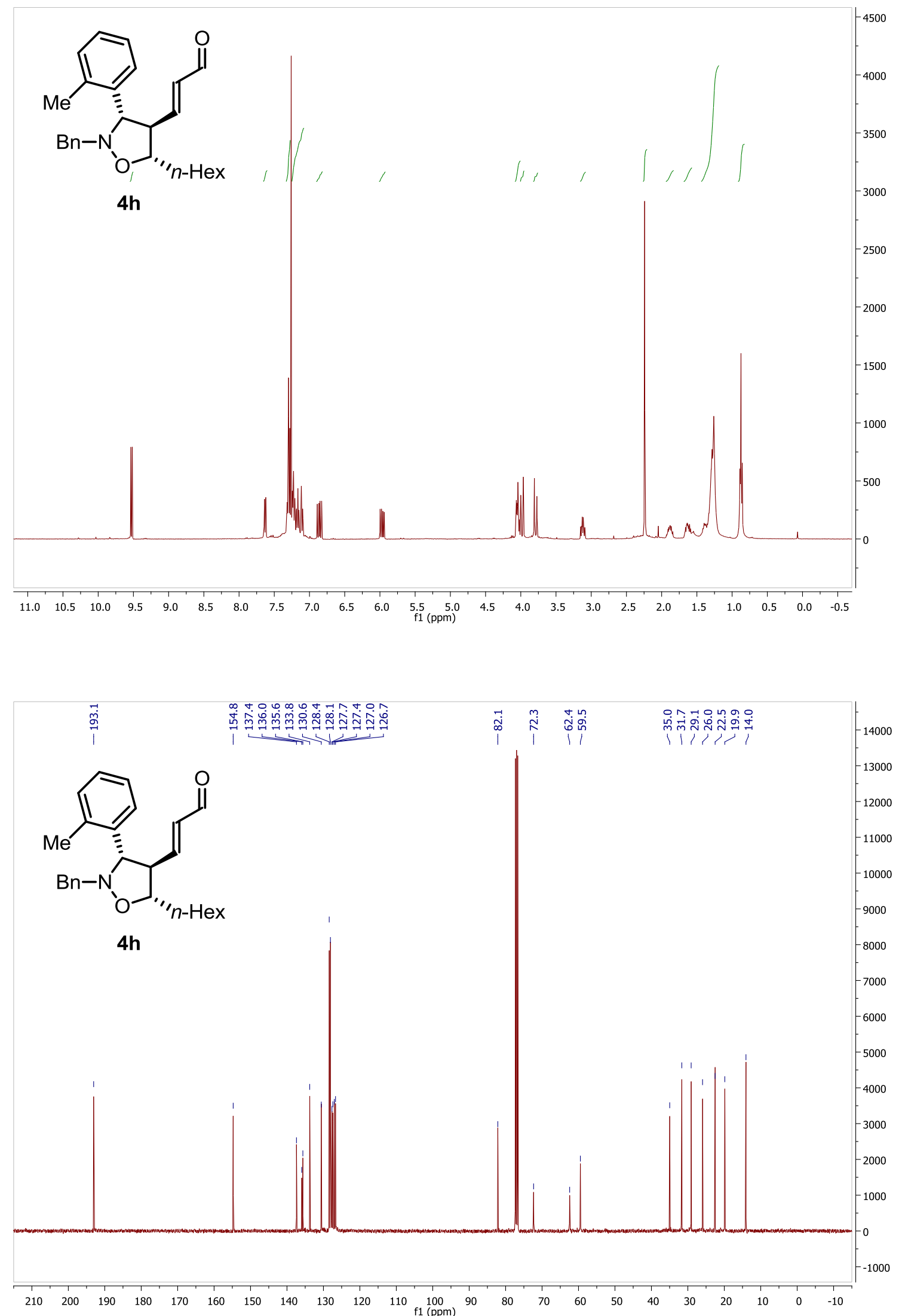

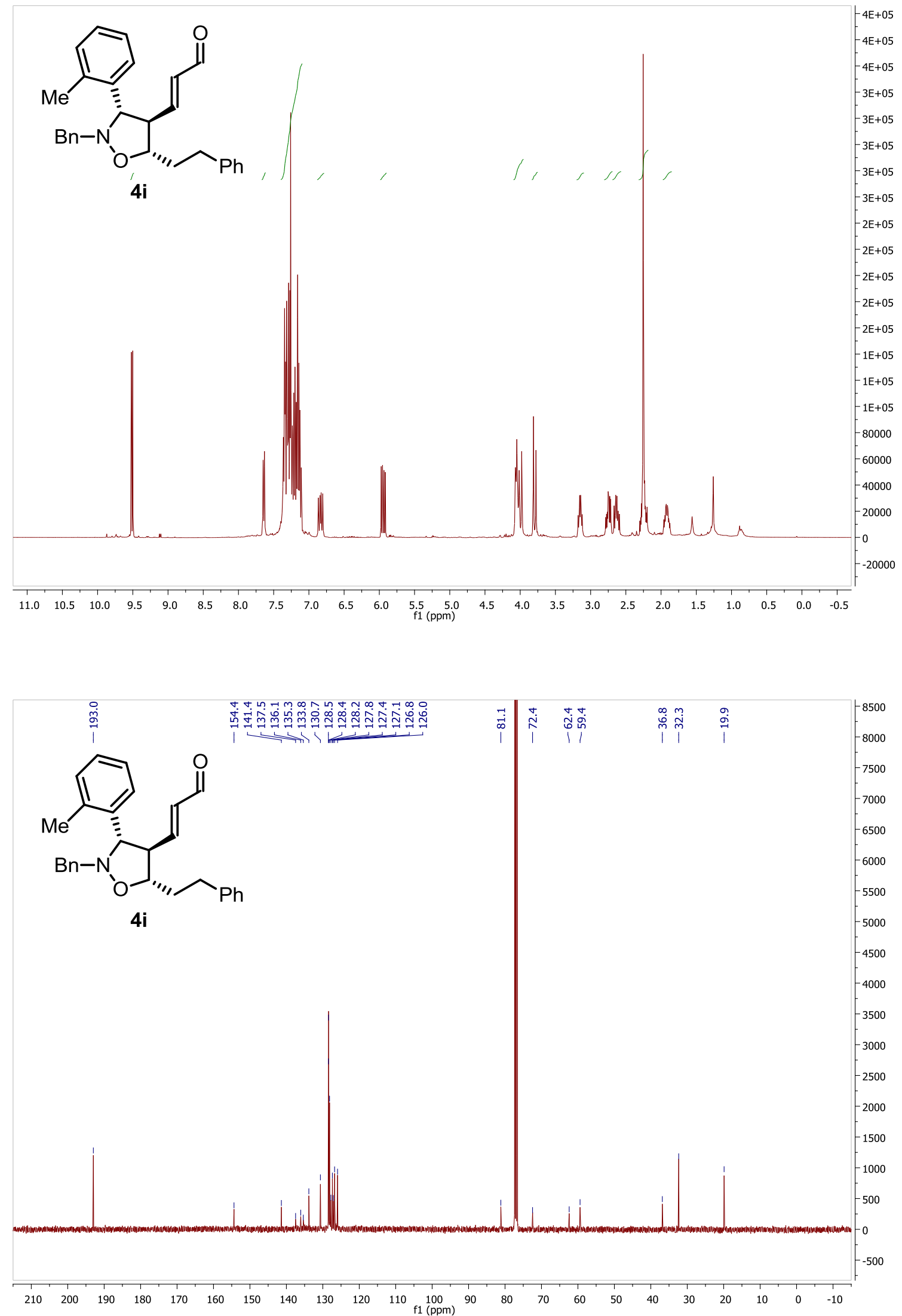

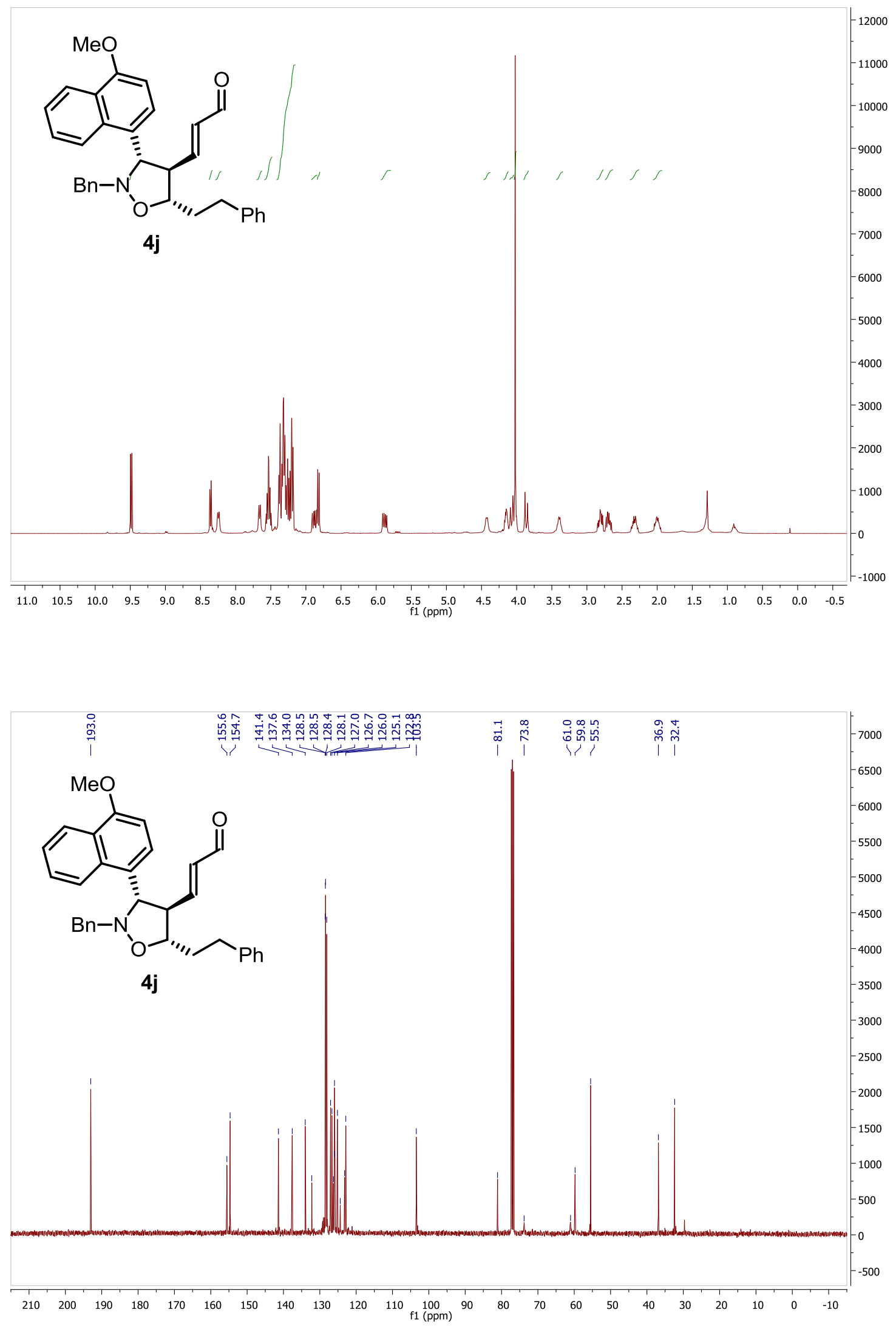

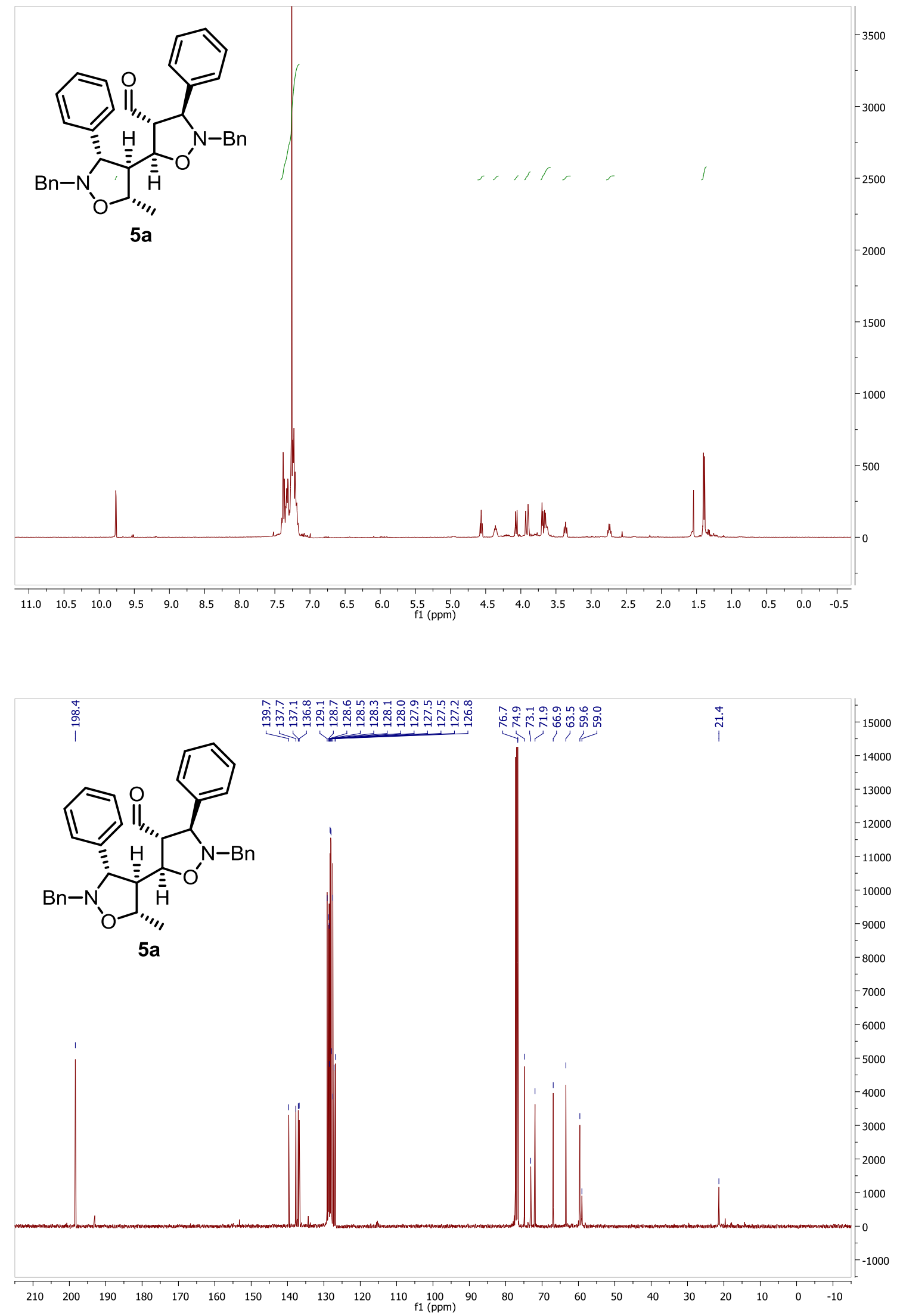

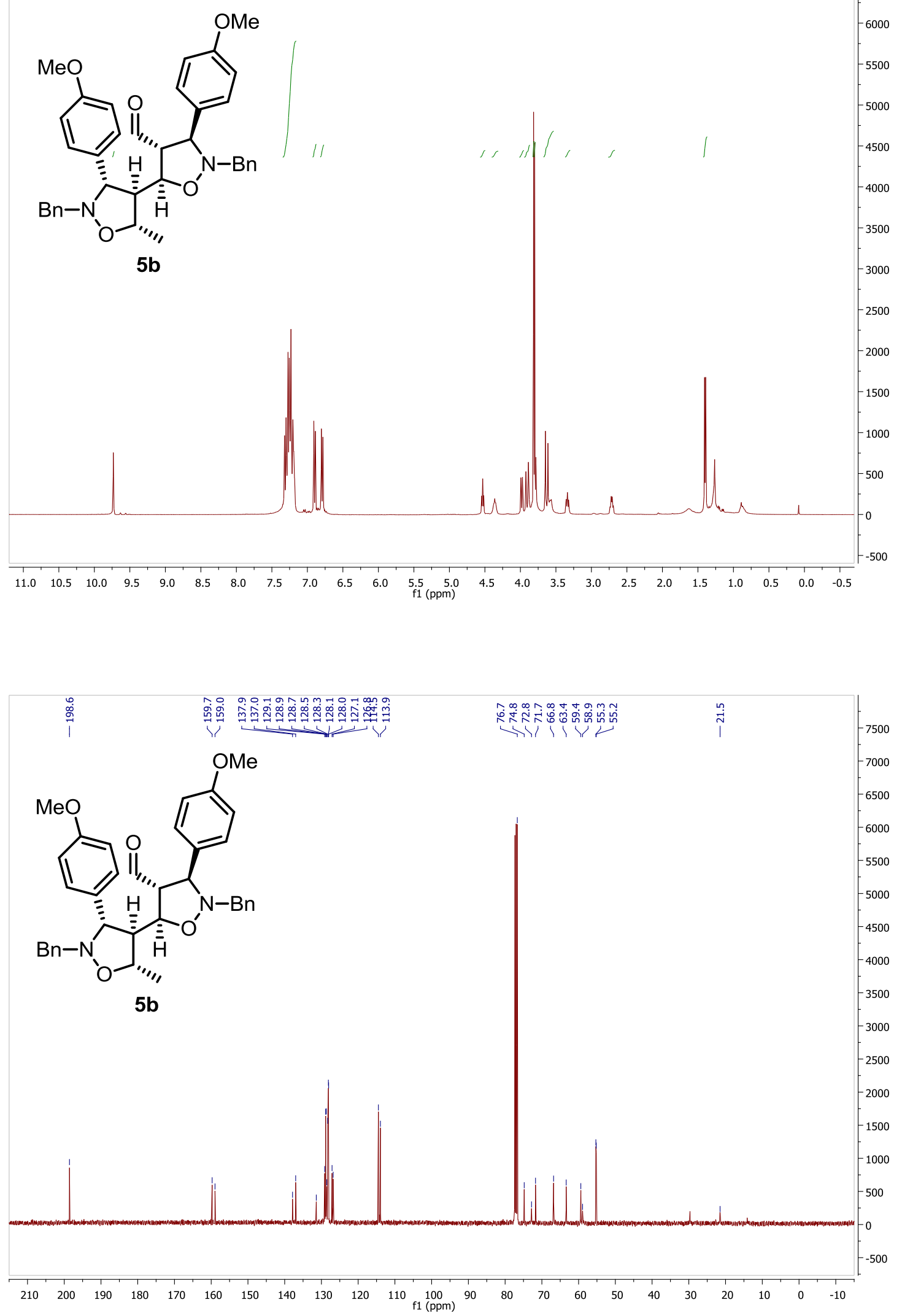

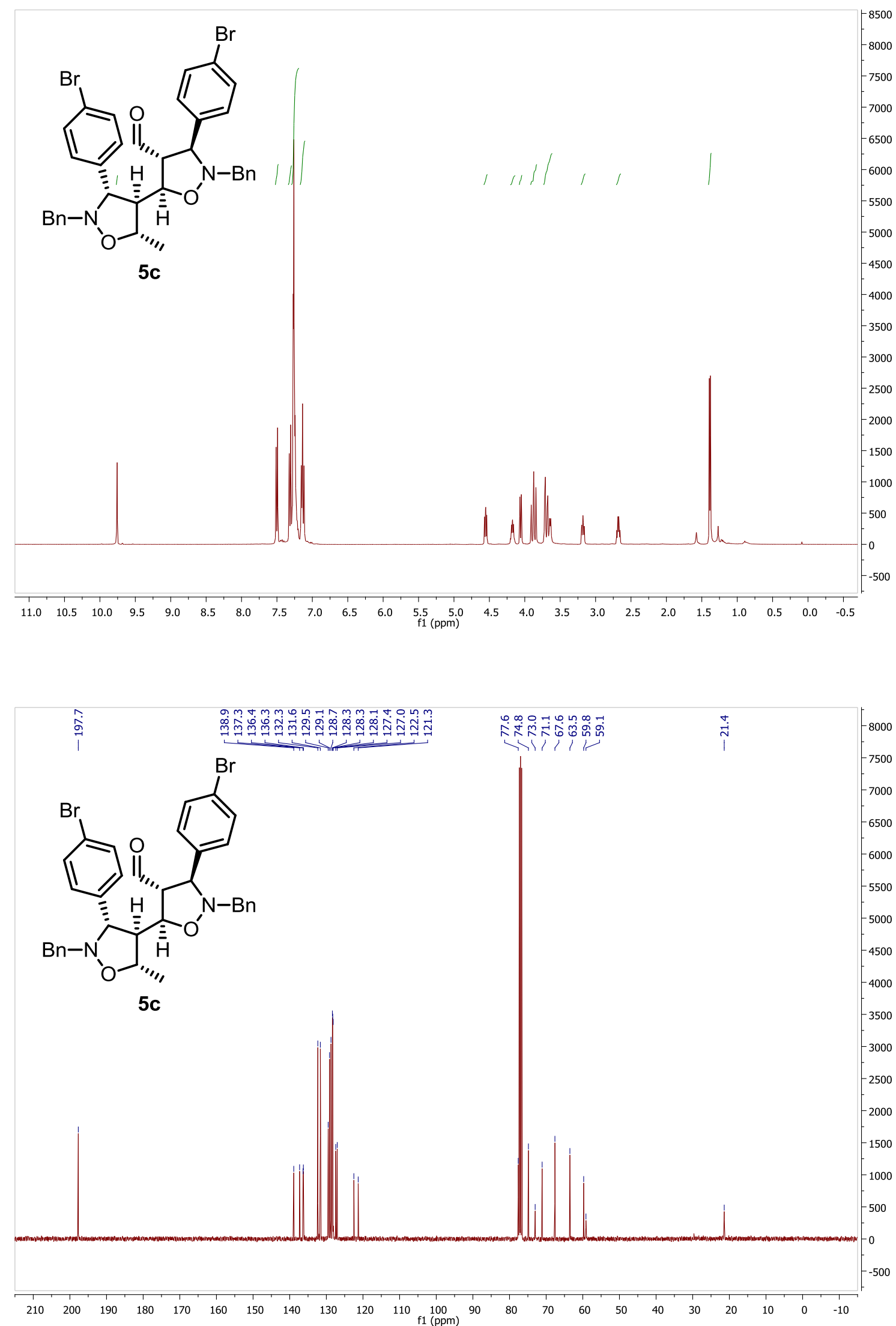

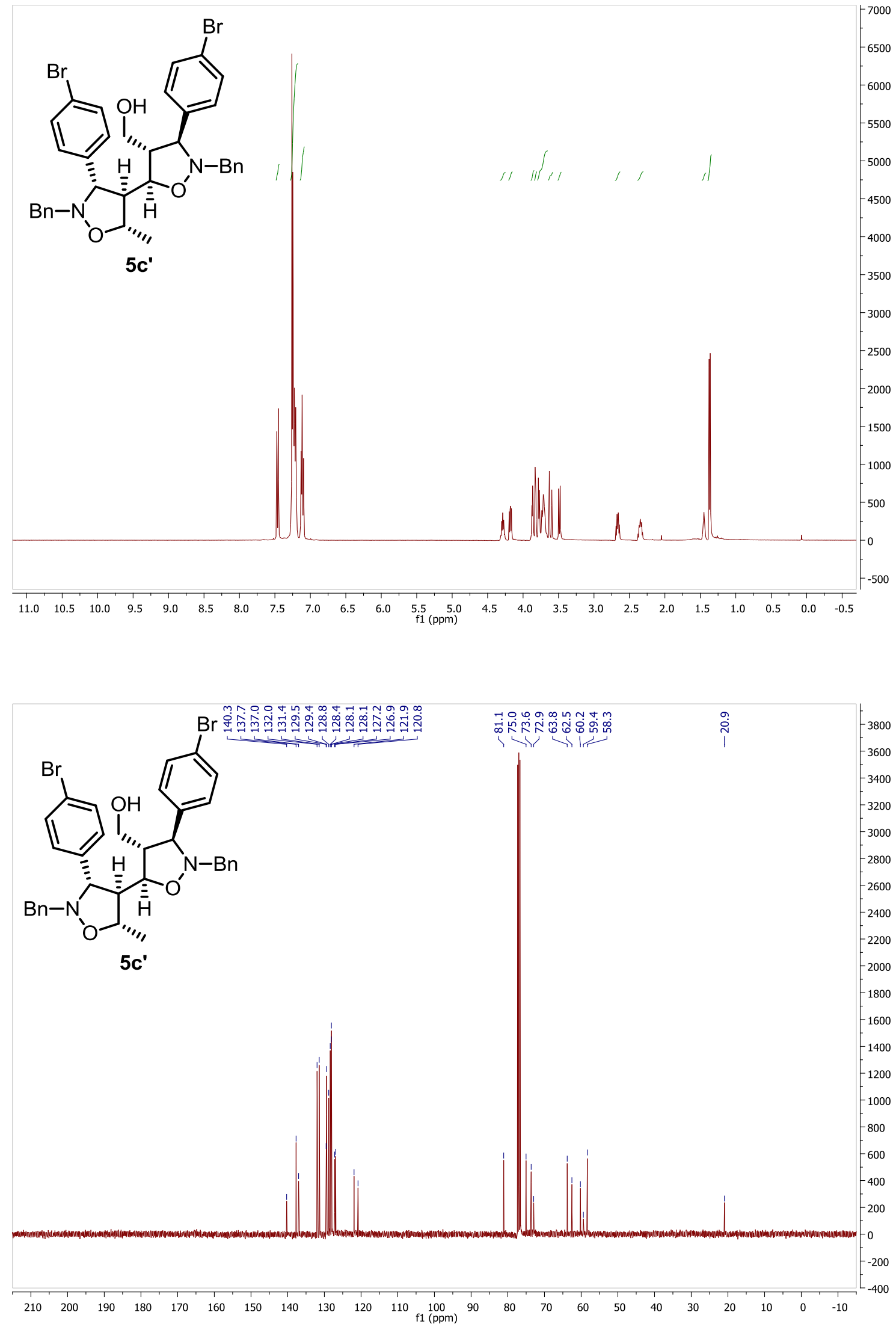

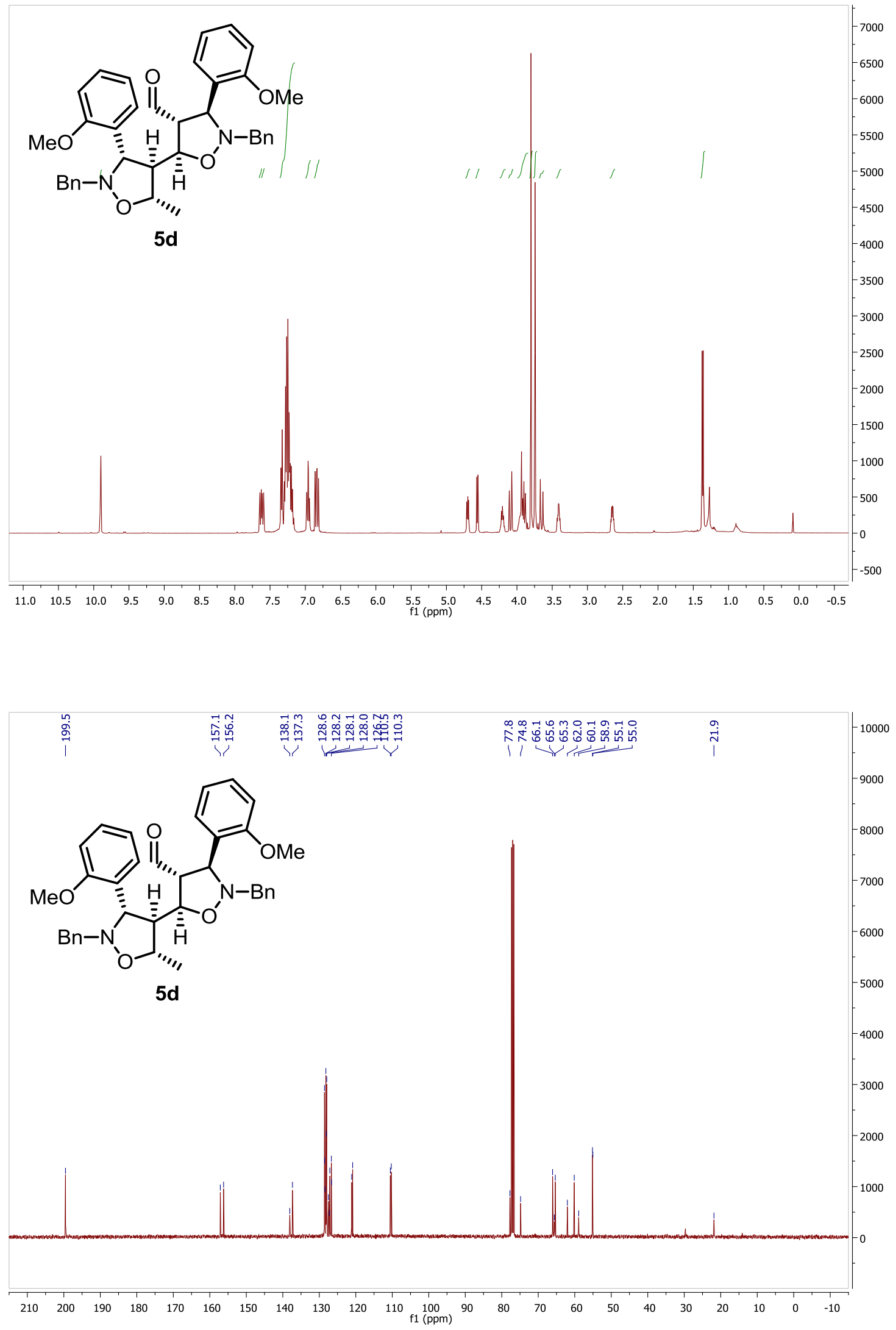

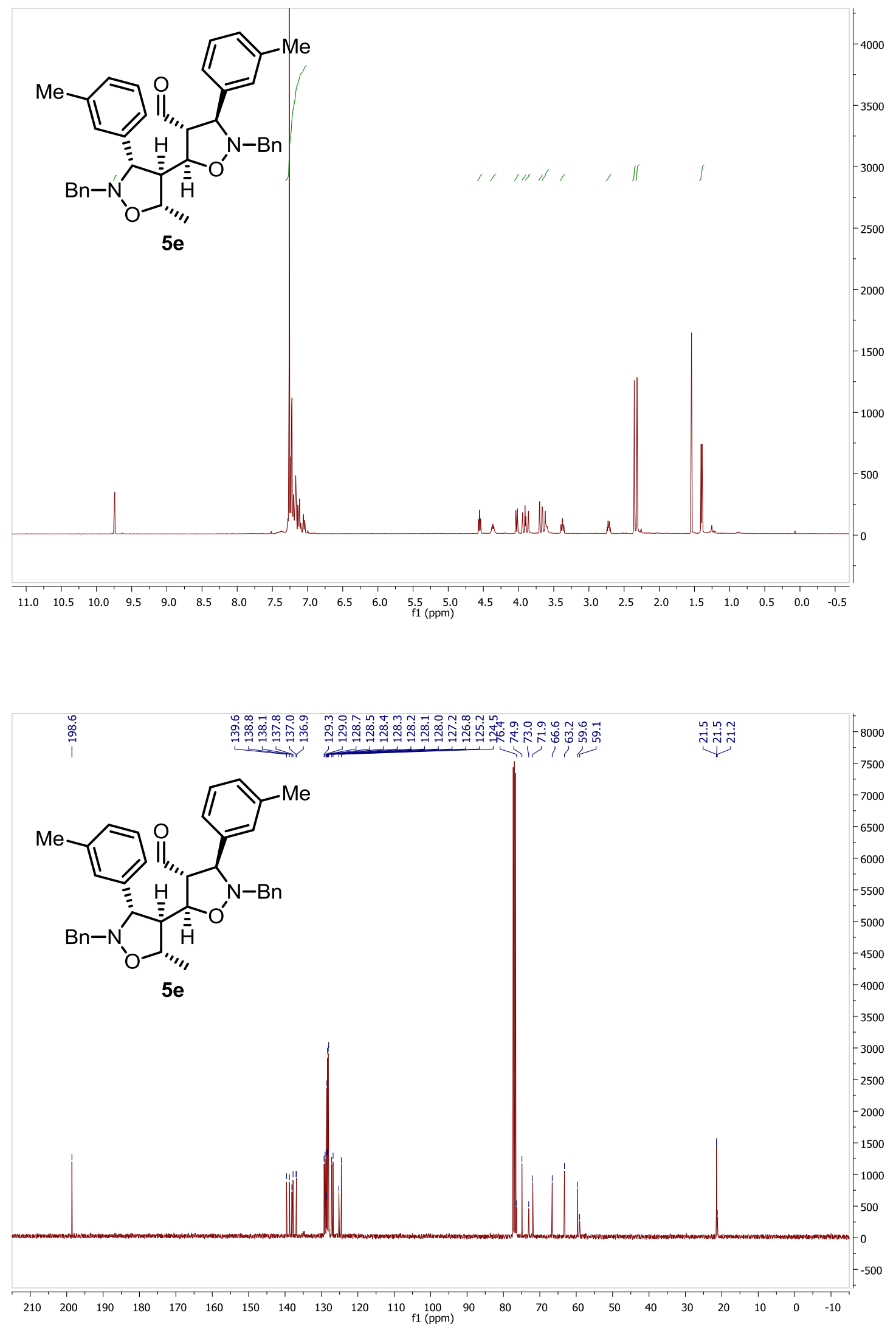

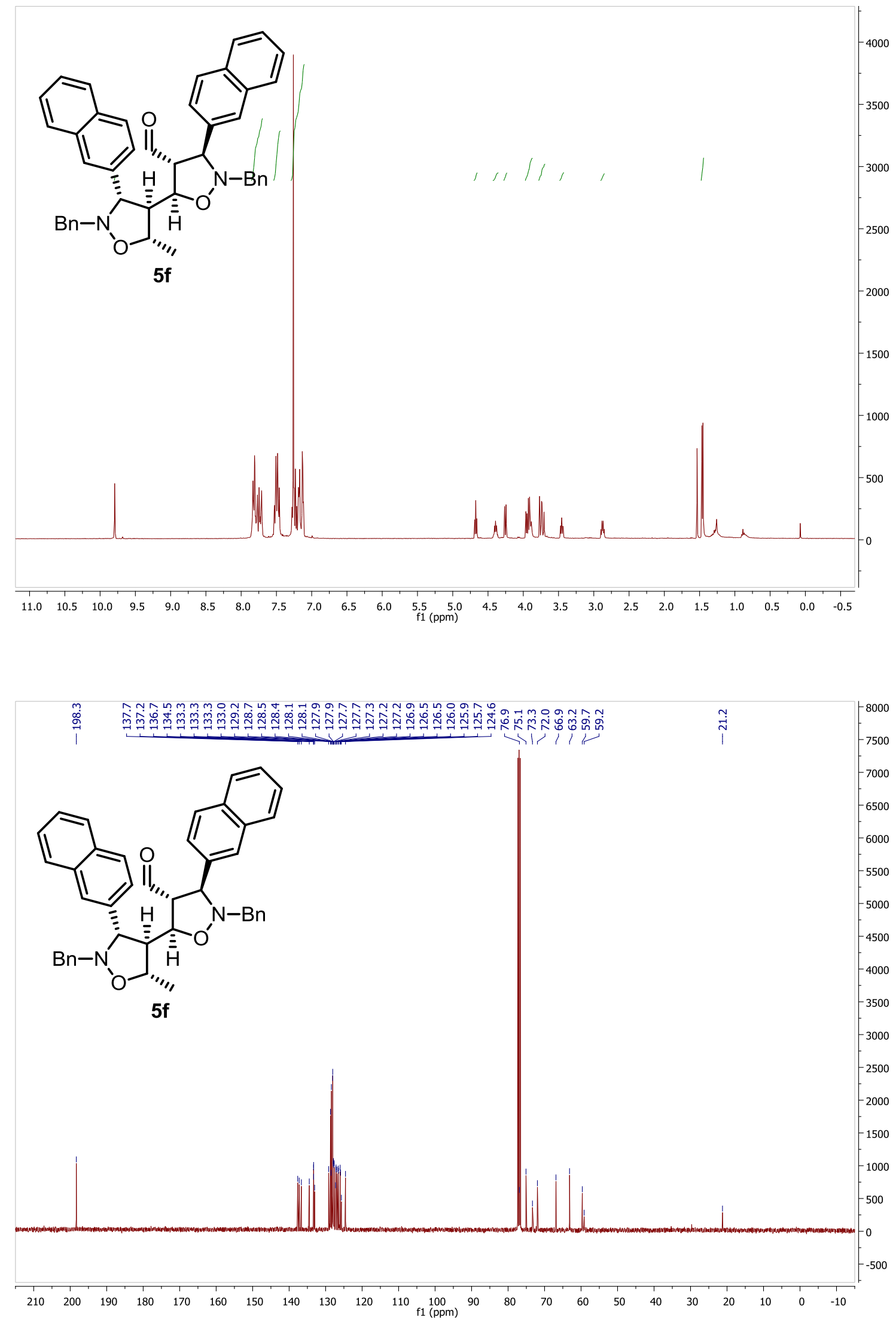

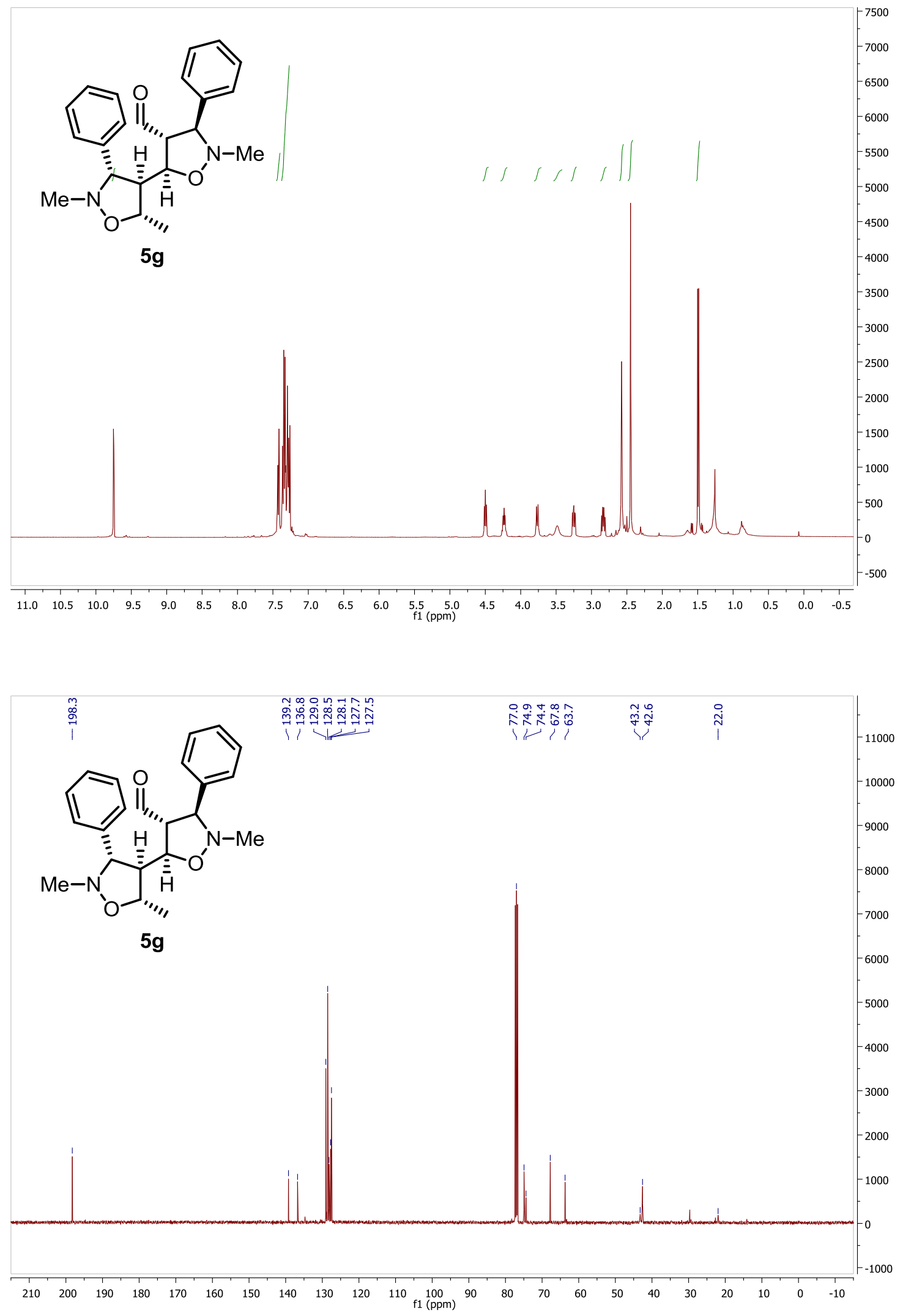

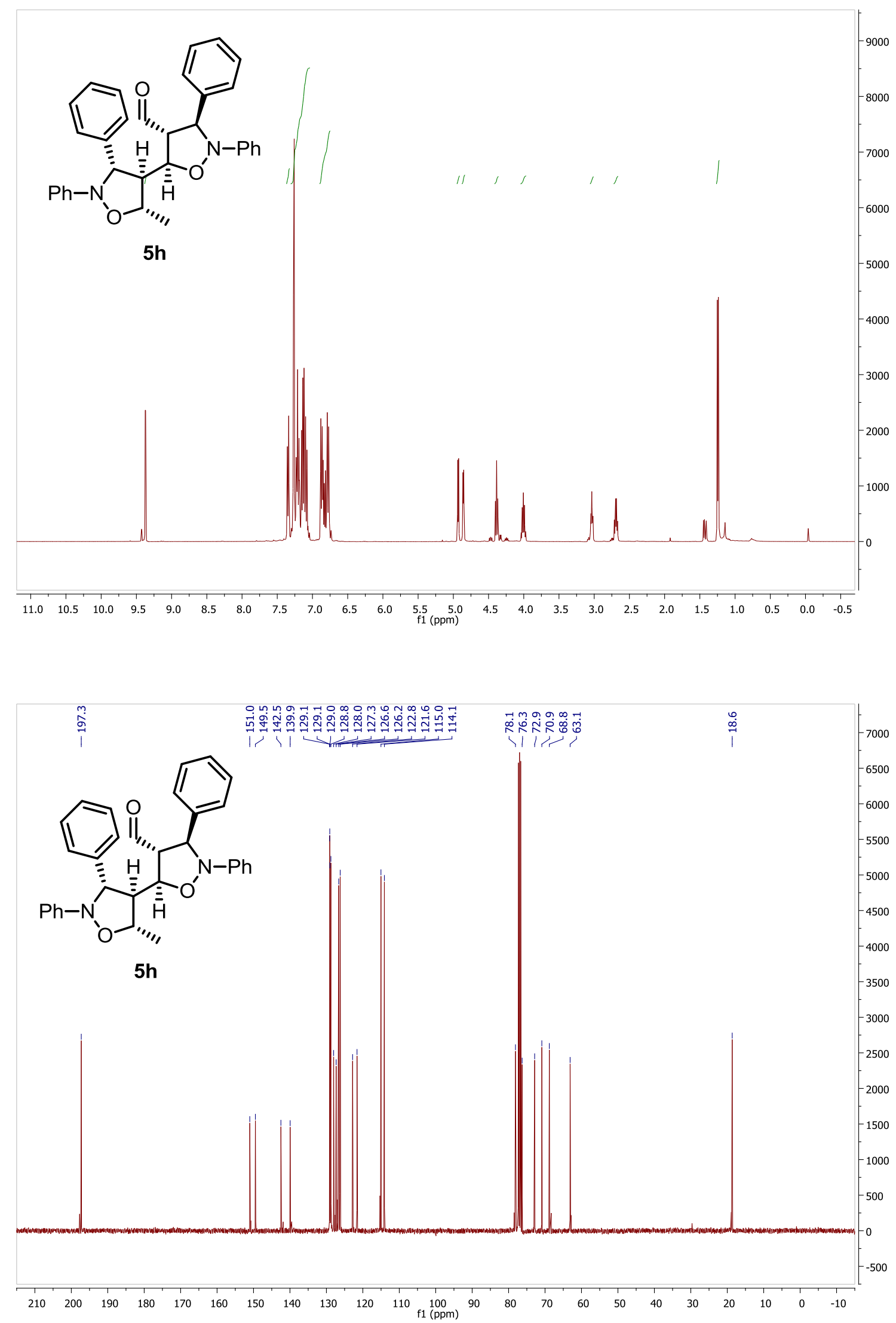


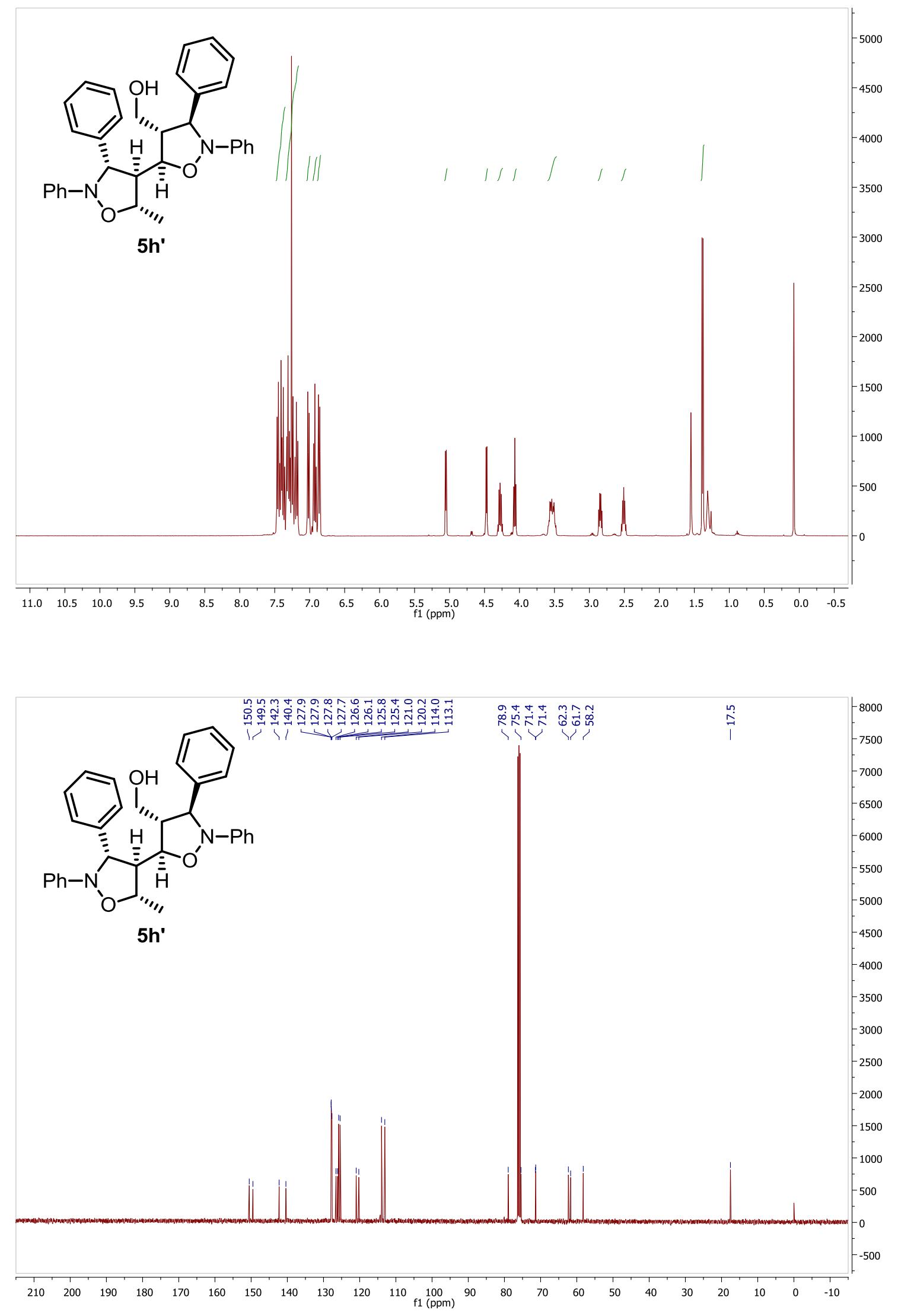



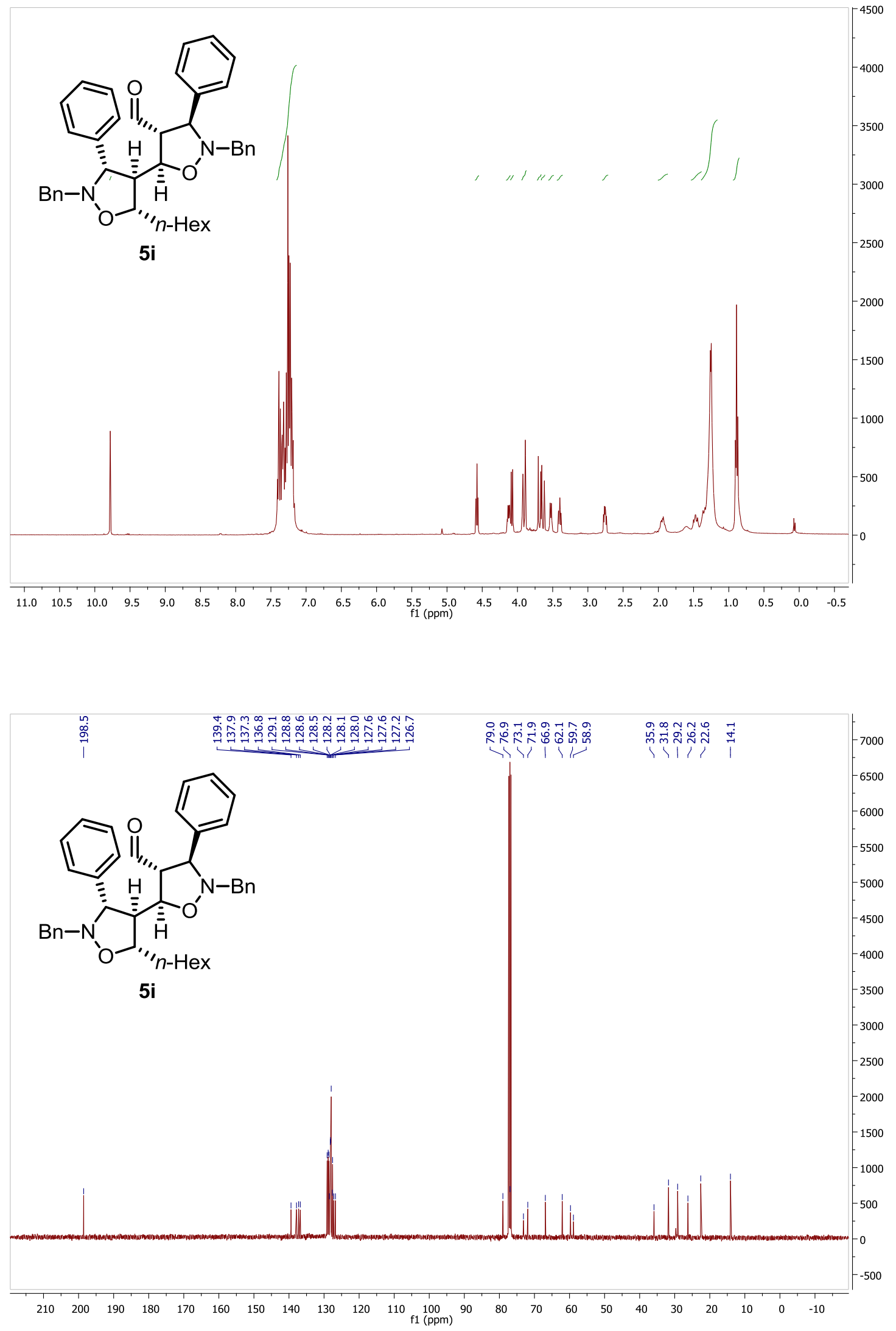

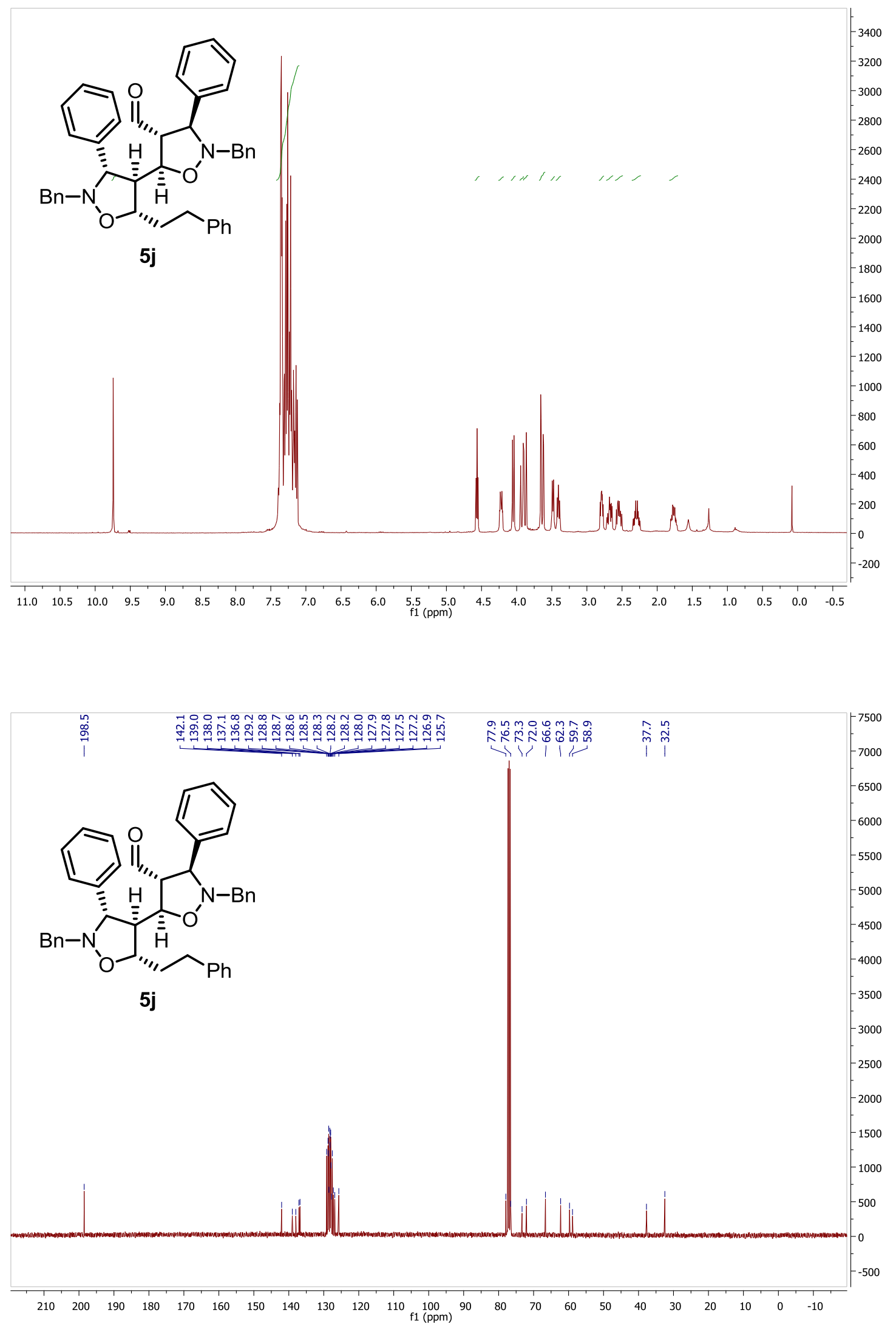

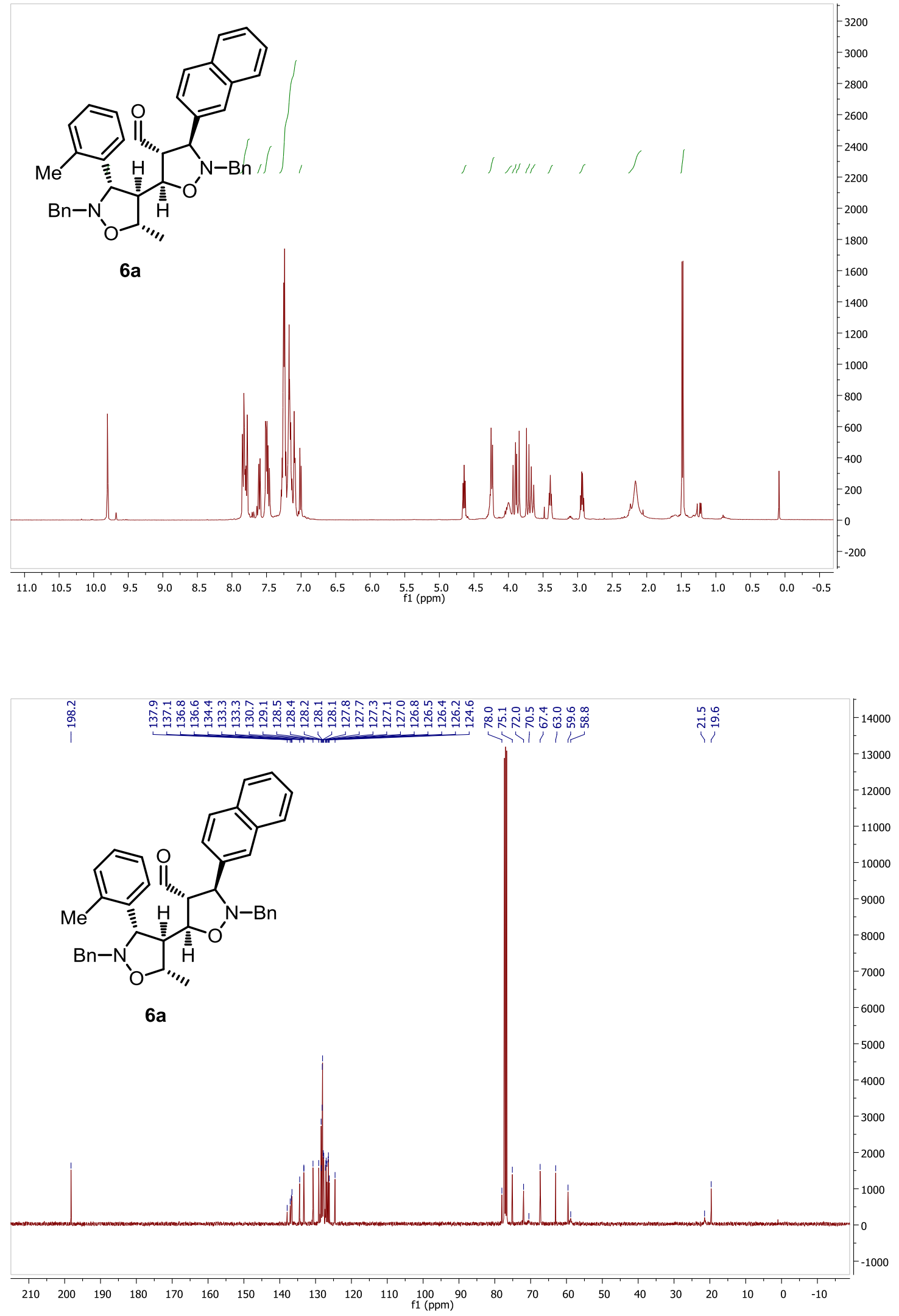

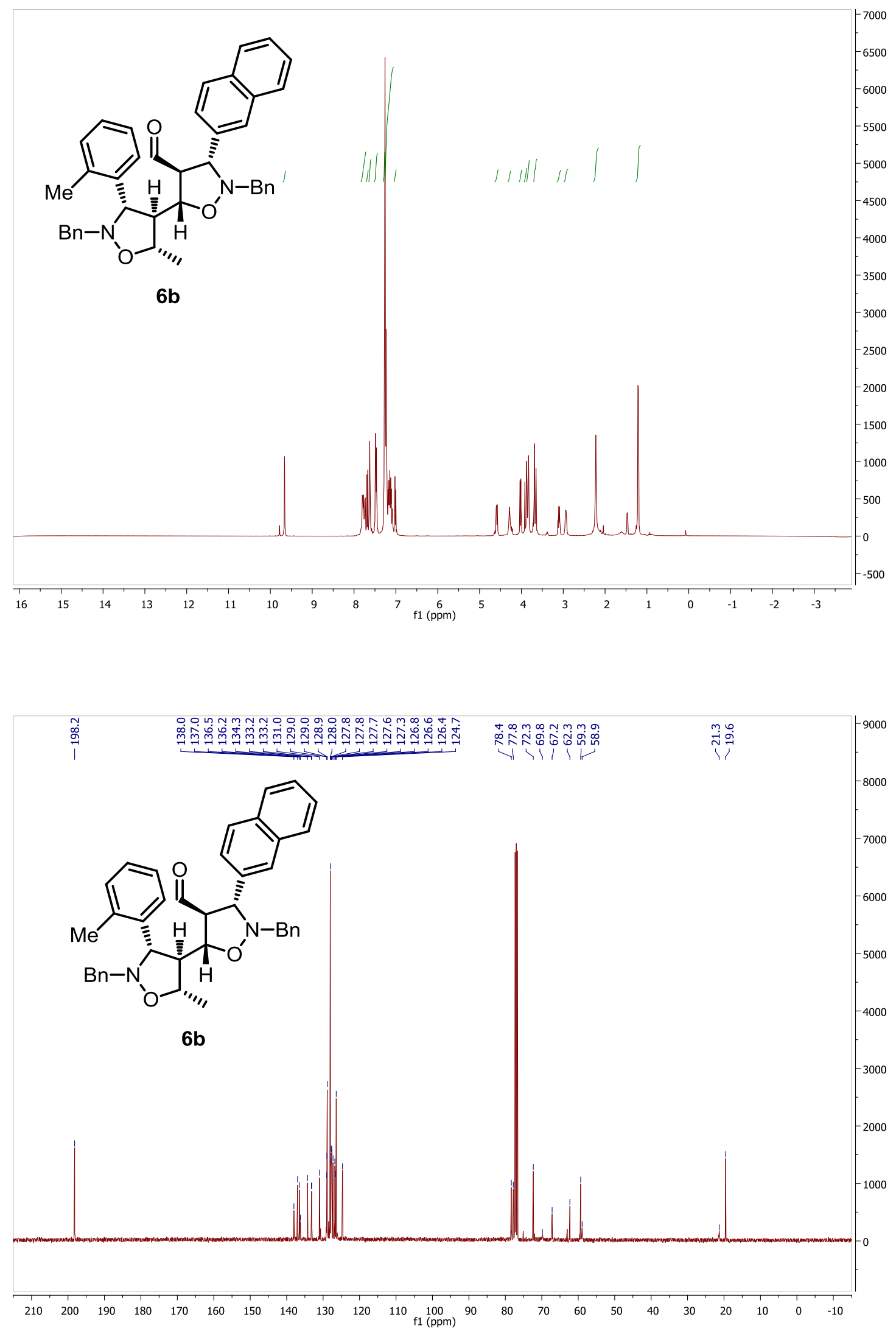

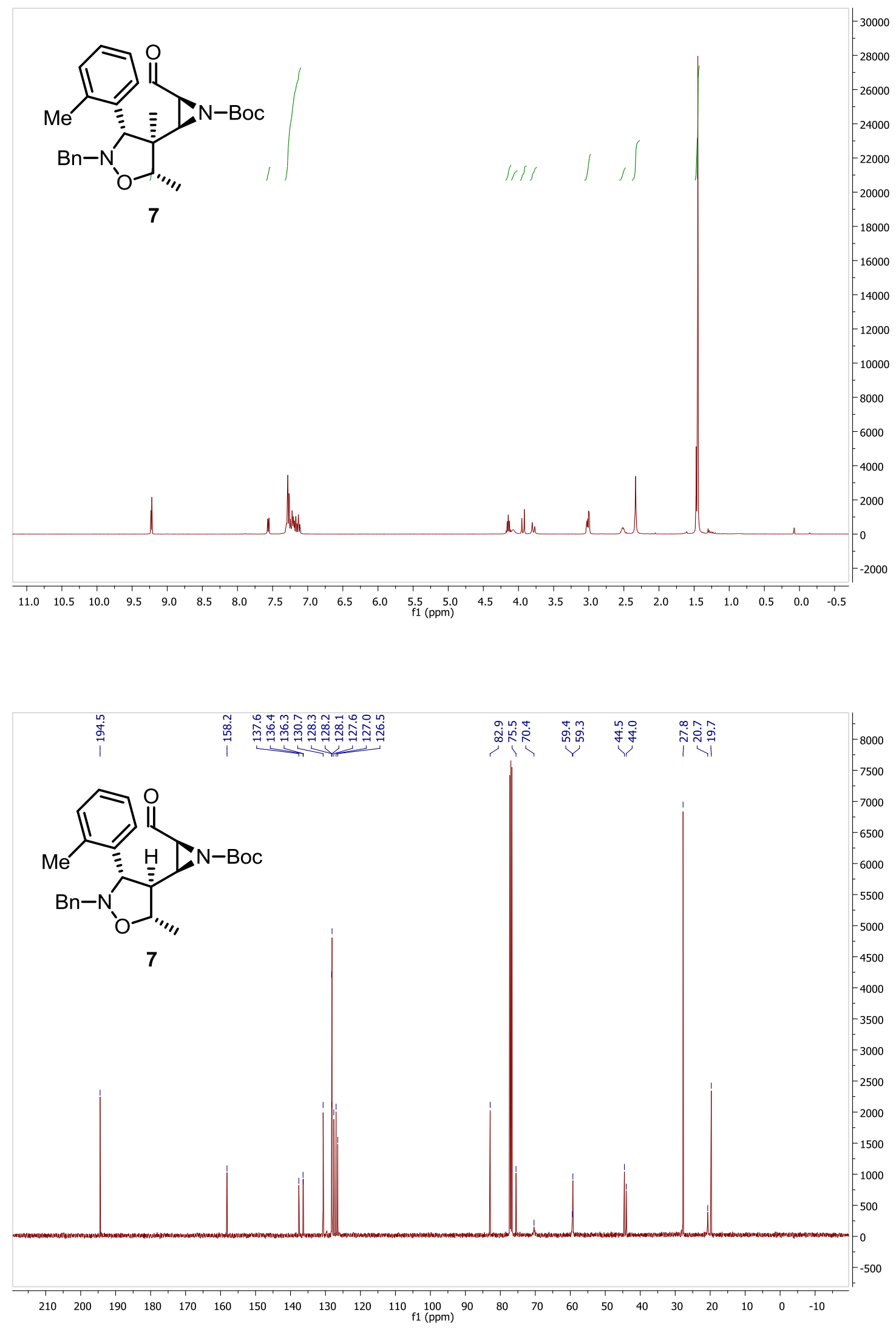

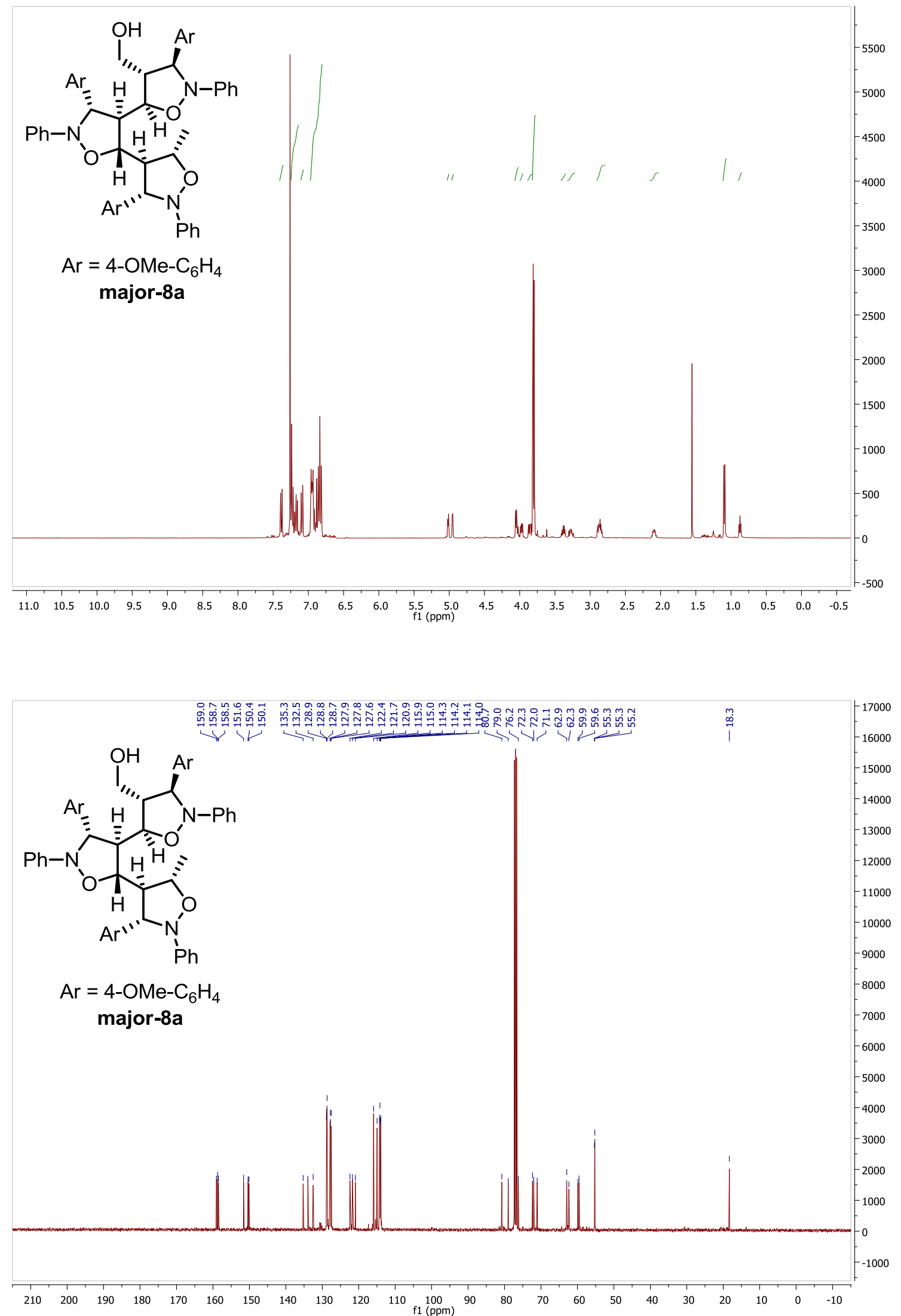

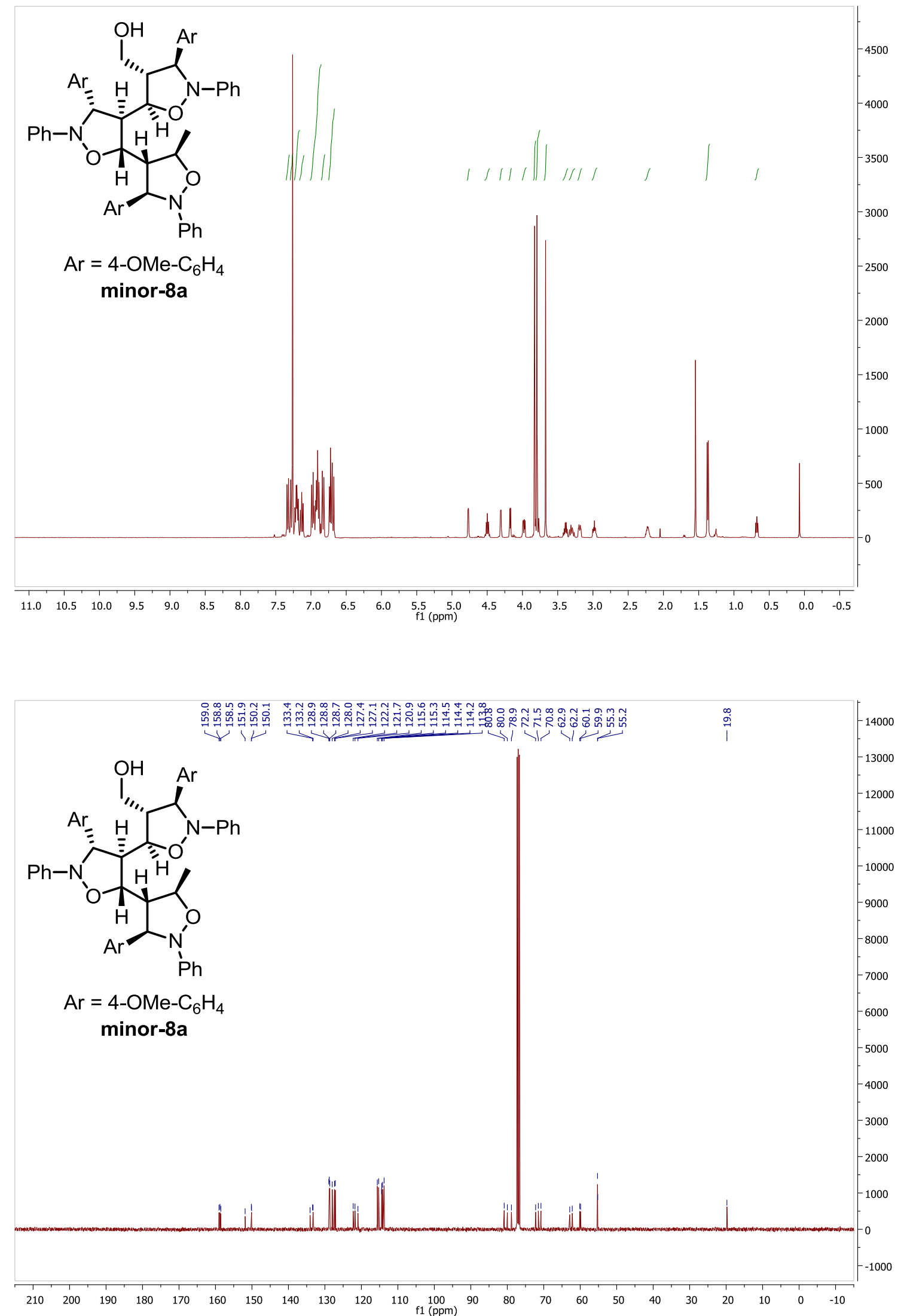

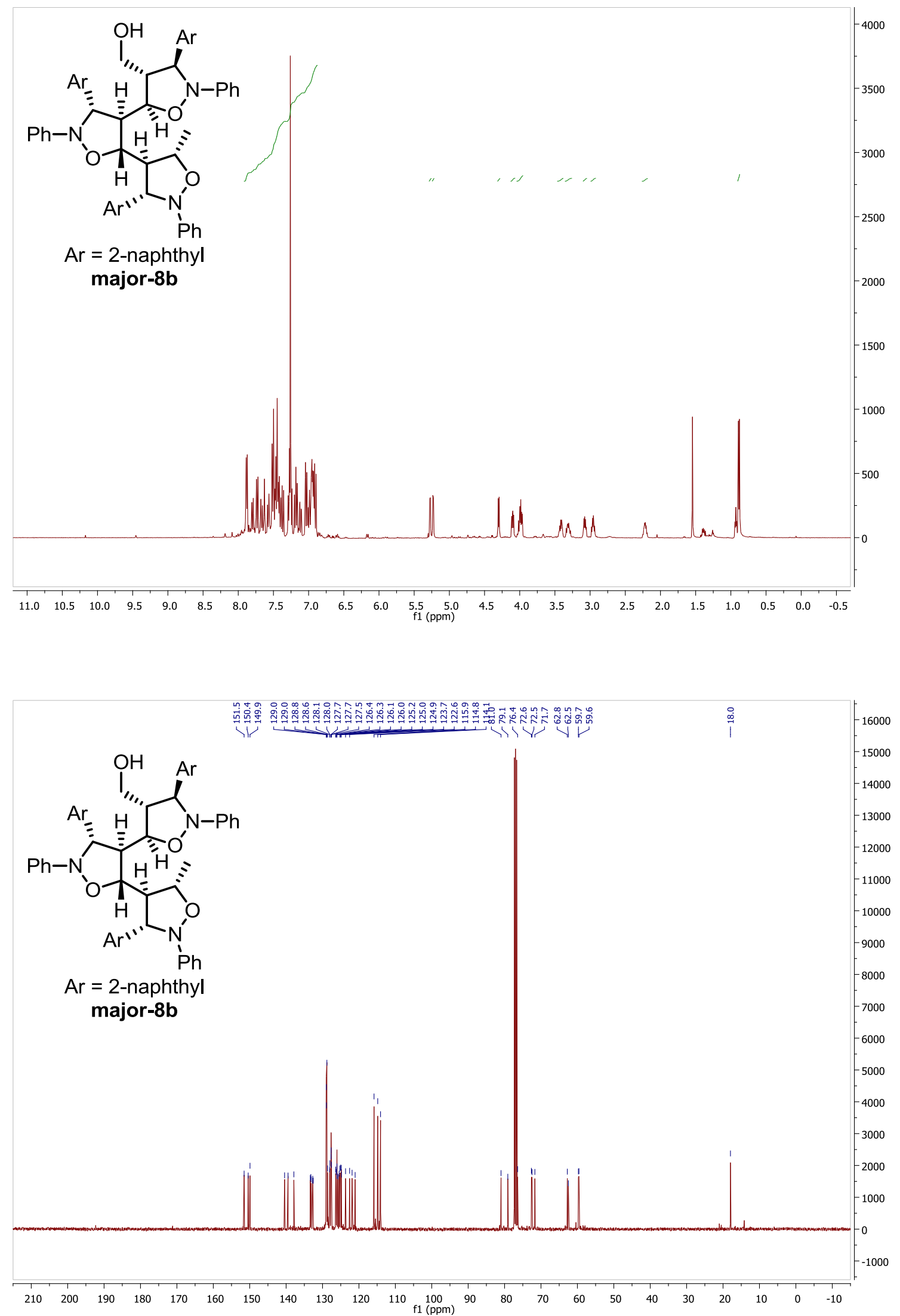

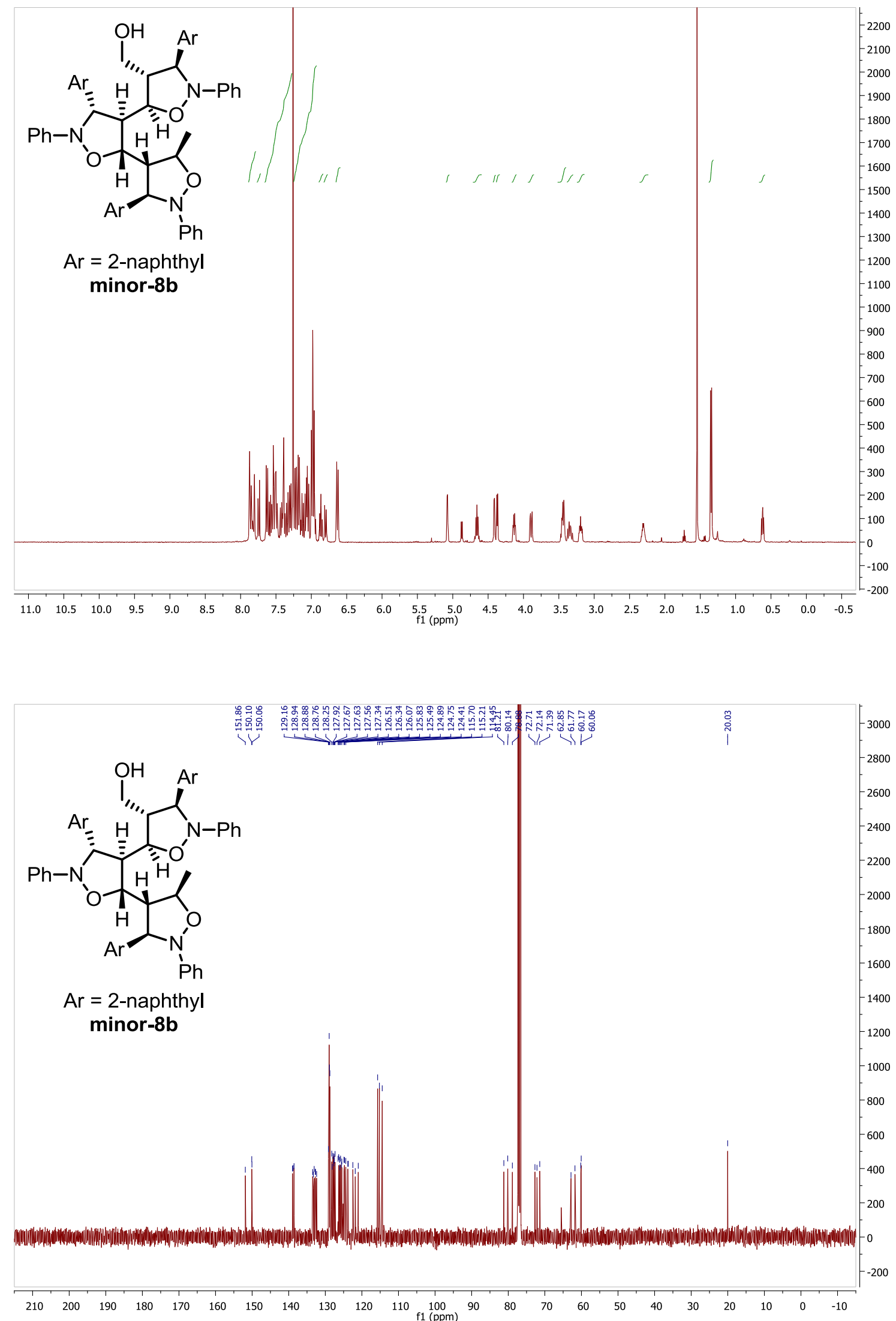

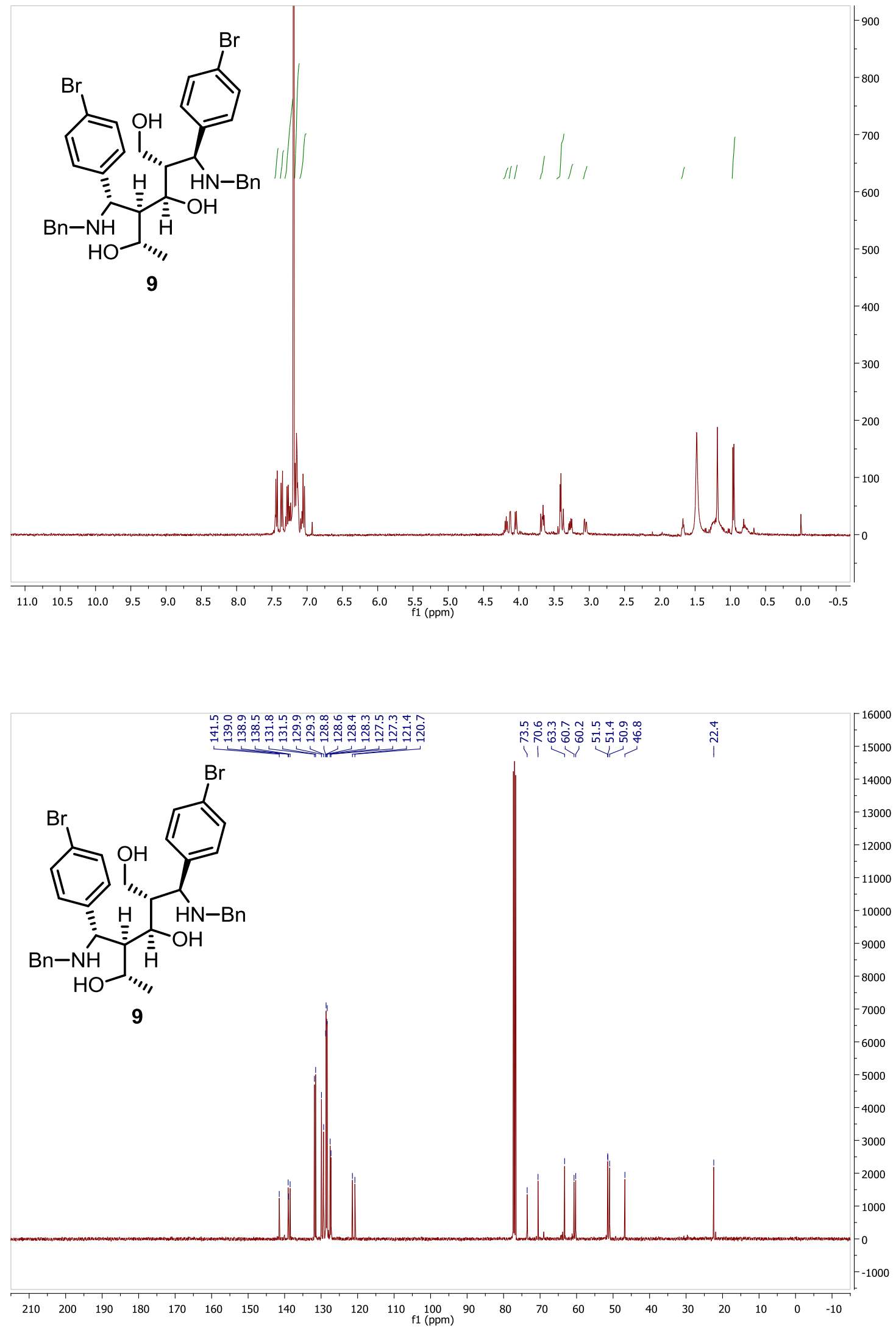


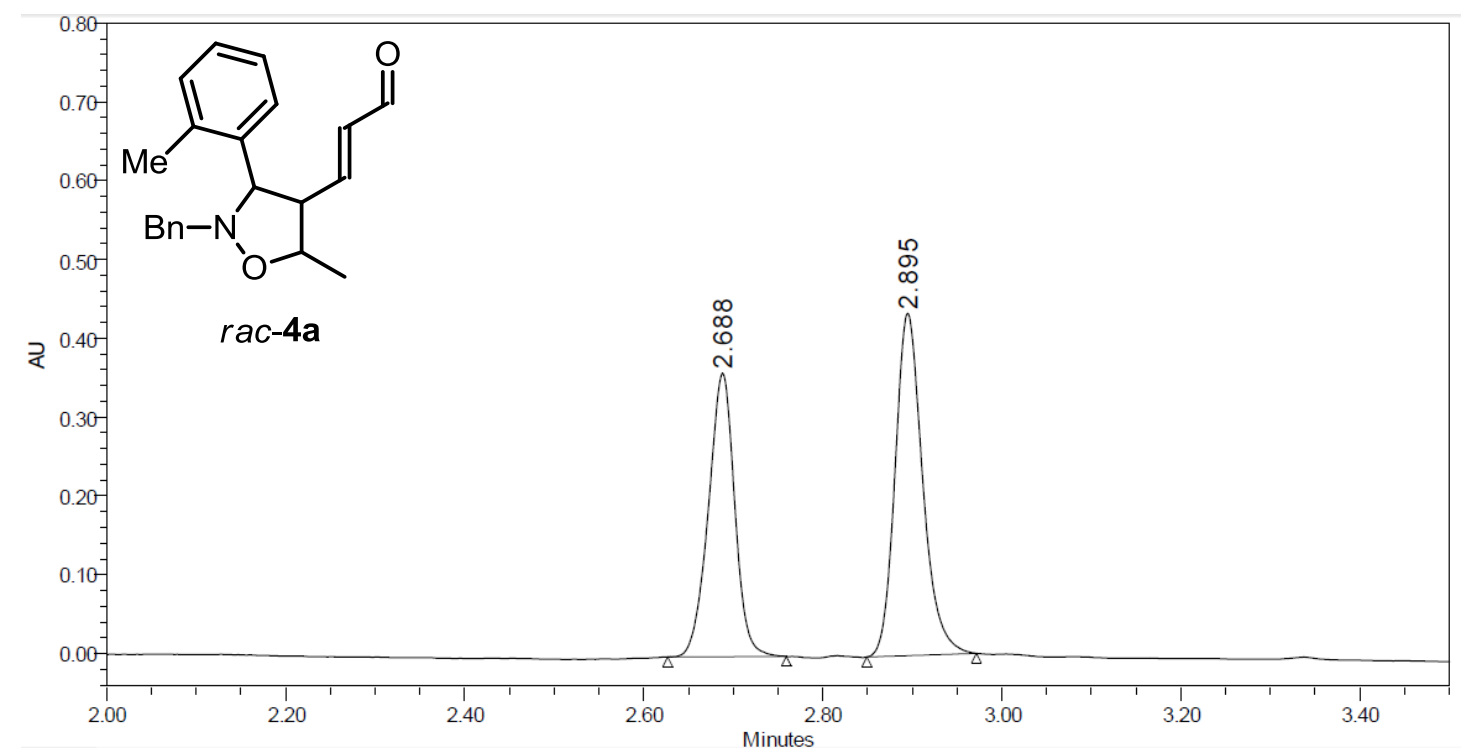

\begin{tabular}{|l|r|r|}
\hline & $\begin{array}{c}\text { Retention Time } \\
\text { (min) }\end{array}$ & \% Area \\
\hline 1 & 2.688 & 44.12 \\
\hline 2 & 2.895 & 55.88 \\
\hline
\end{tabular}

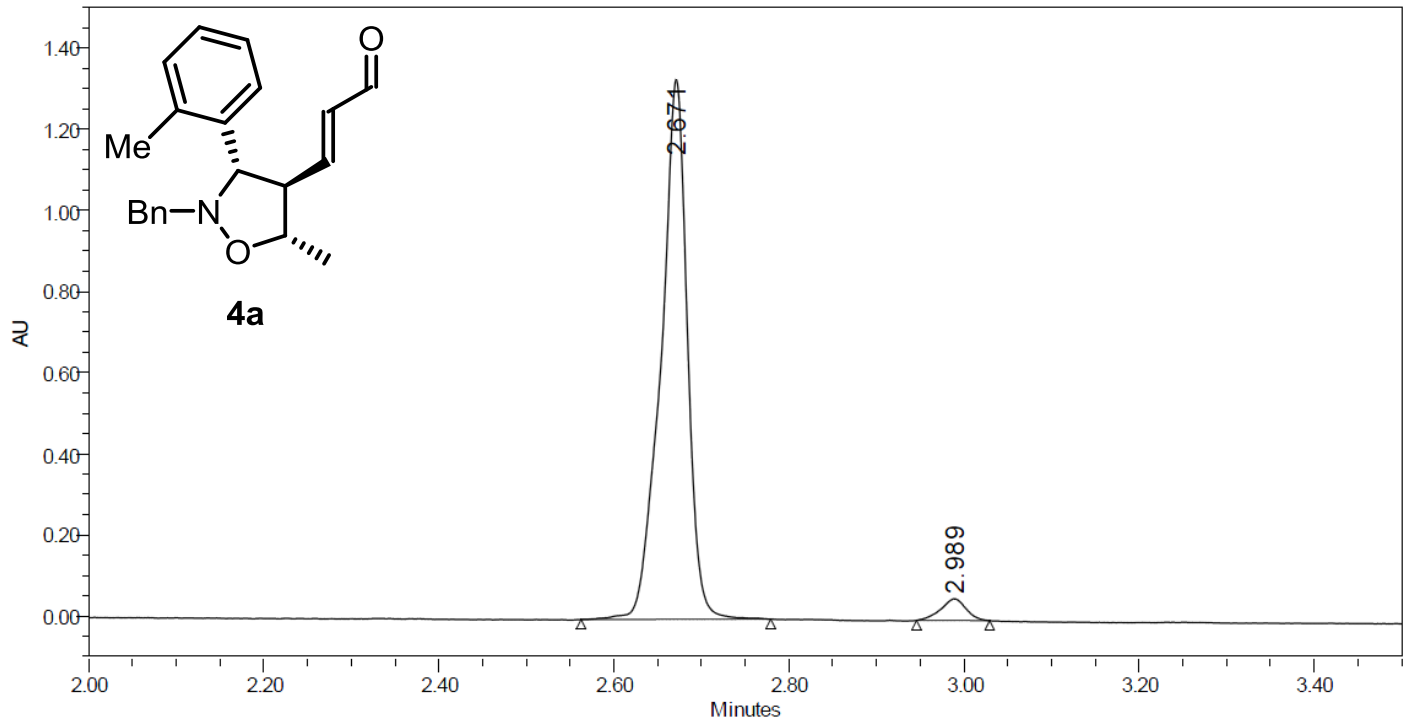

\begin{tabular}{|r|r|r|}
\hline & $\begin{array}{c}\text { Retention Time } \\
\text { (min) }\end{array}$ & \% Area \\
\hline 1 & 2.671 & 96.32 \\
\hline 2 & 2.989 & 3.68 \\
\hline
\end{tabular}



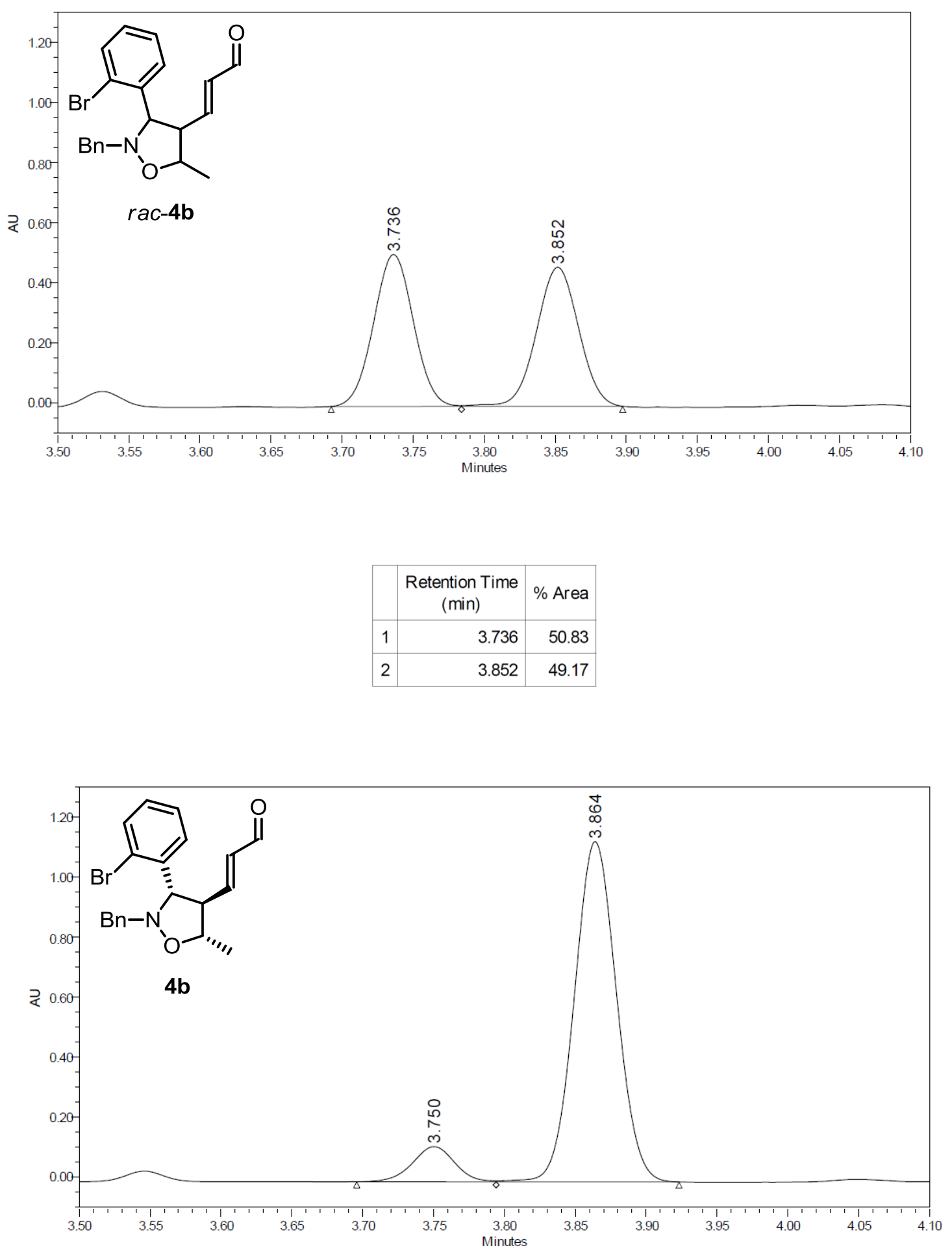


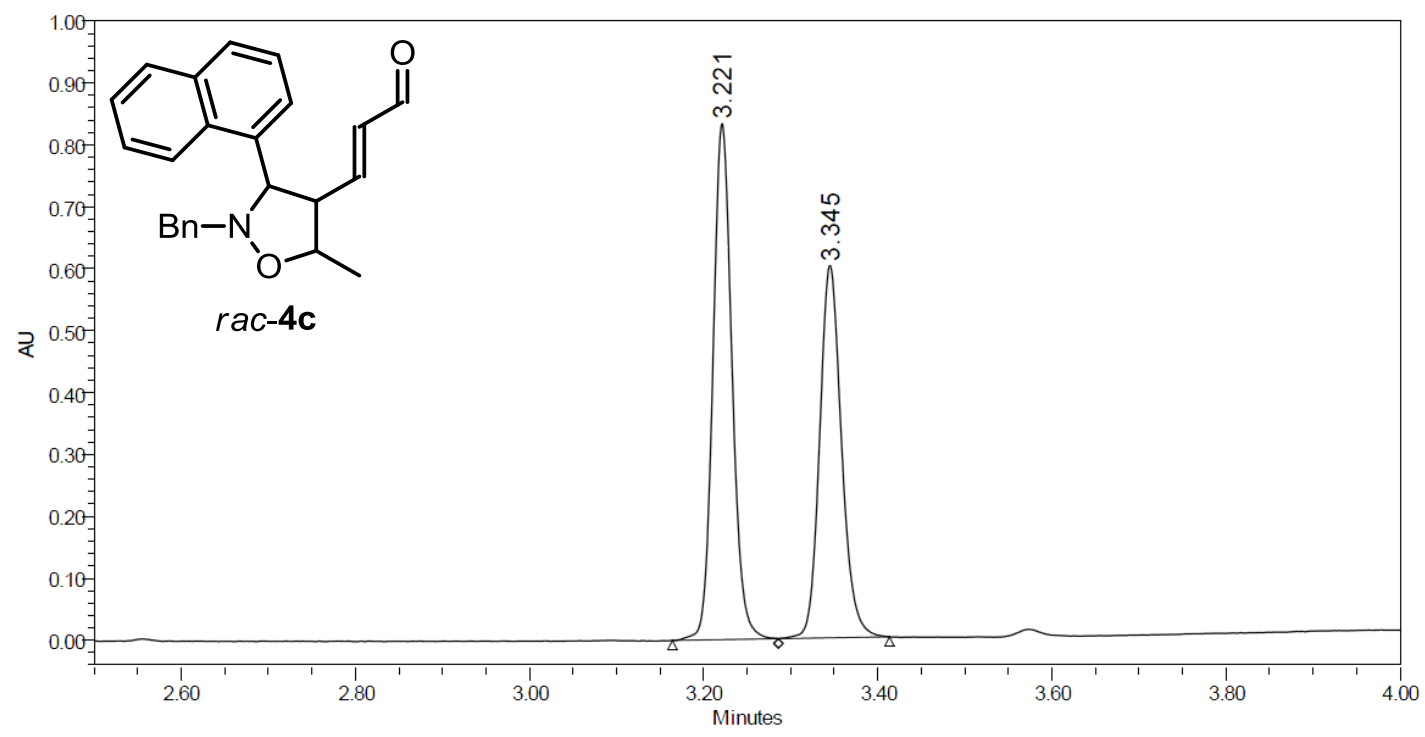

\begin{tabular}{|l|r|r|}
\hline & $\begin{array}{r}\text { Retention Time } \\
(\mathrm{min})\end{array}$ & $\%$ Area \\
\hline 1 & 3.221 & 54.73 \\
\hline 2 & 3.345 & 45.27 \\
\hline
\end{tabular}

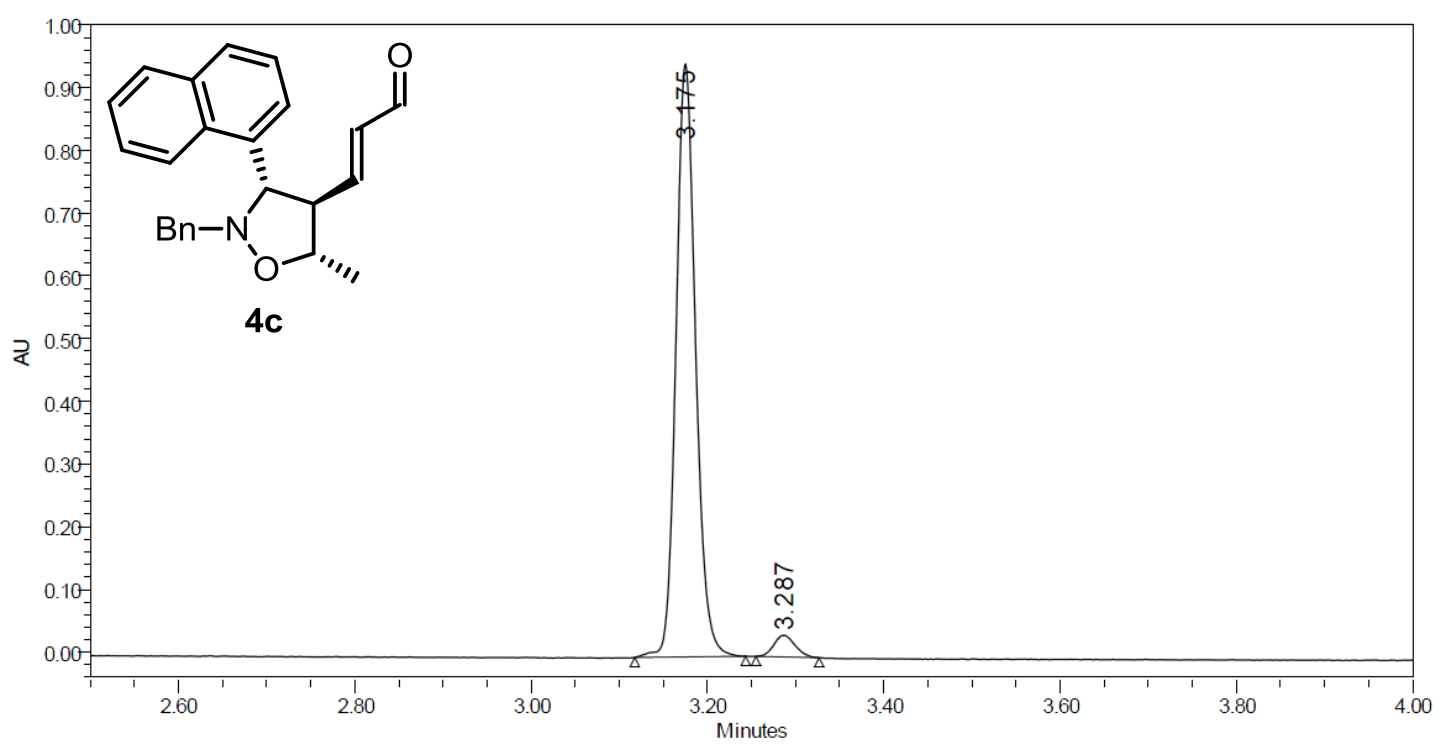

\begin{tabular}{|l|r|r|}
\hline & $\begin{array}{c}\text { Retention Time } \\
(\mathrm{min})\end{array}$ & \% Area \\
\hline 1 & 3.175 & 96.20 \\
\hline 2 & 3.287 & 3.80 \\
\hline
\end{tabular}




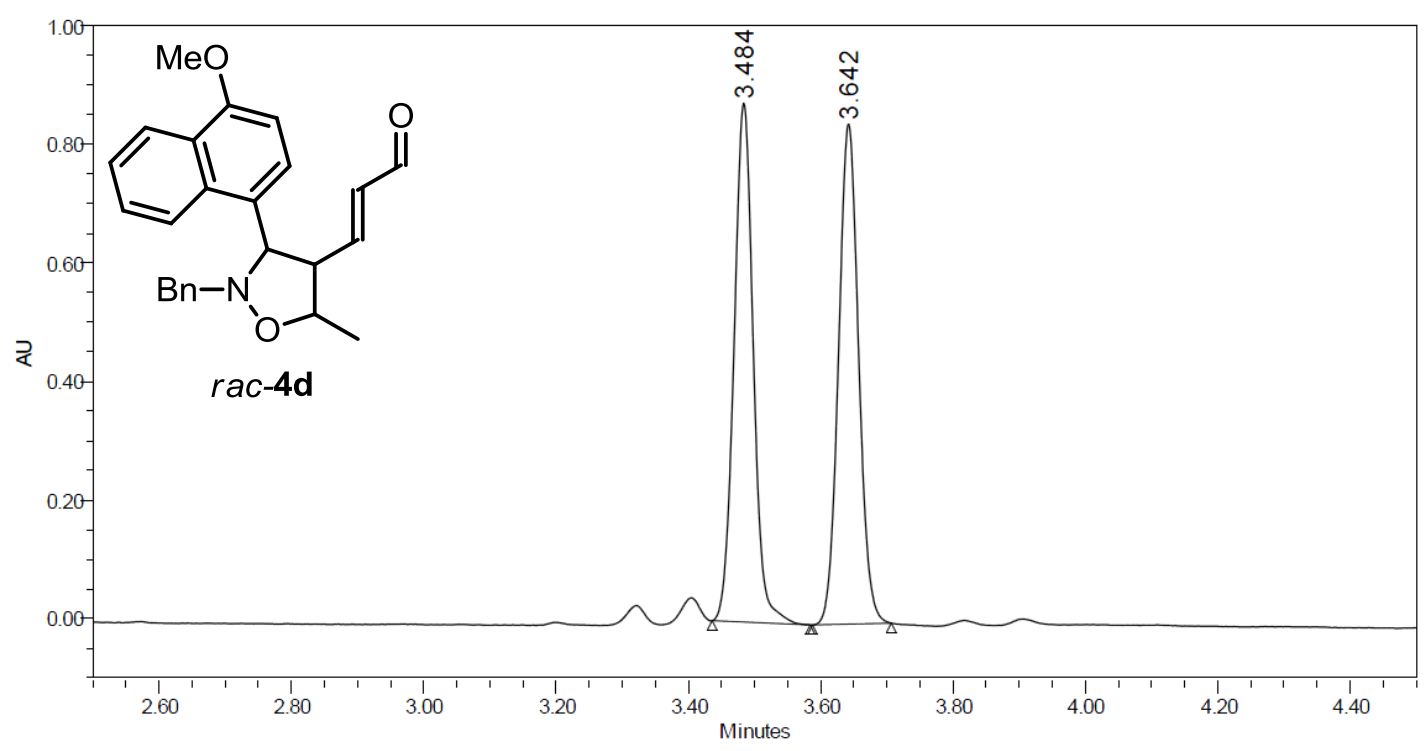

\begin{tabular}{|l|r|r|}
\hline & $\begin{array}{c}\text { Retention Time } \\
\text { (min) }\end{array}$ & \% Area \\
\hline 1 & 3.484 & 49.89 \\
\hline 2 & 3.642 & 50.11 \\
\hline
\end{tabular}

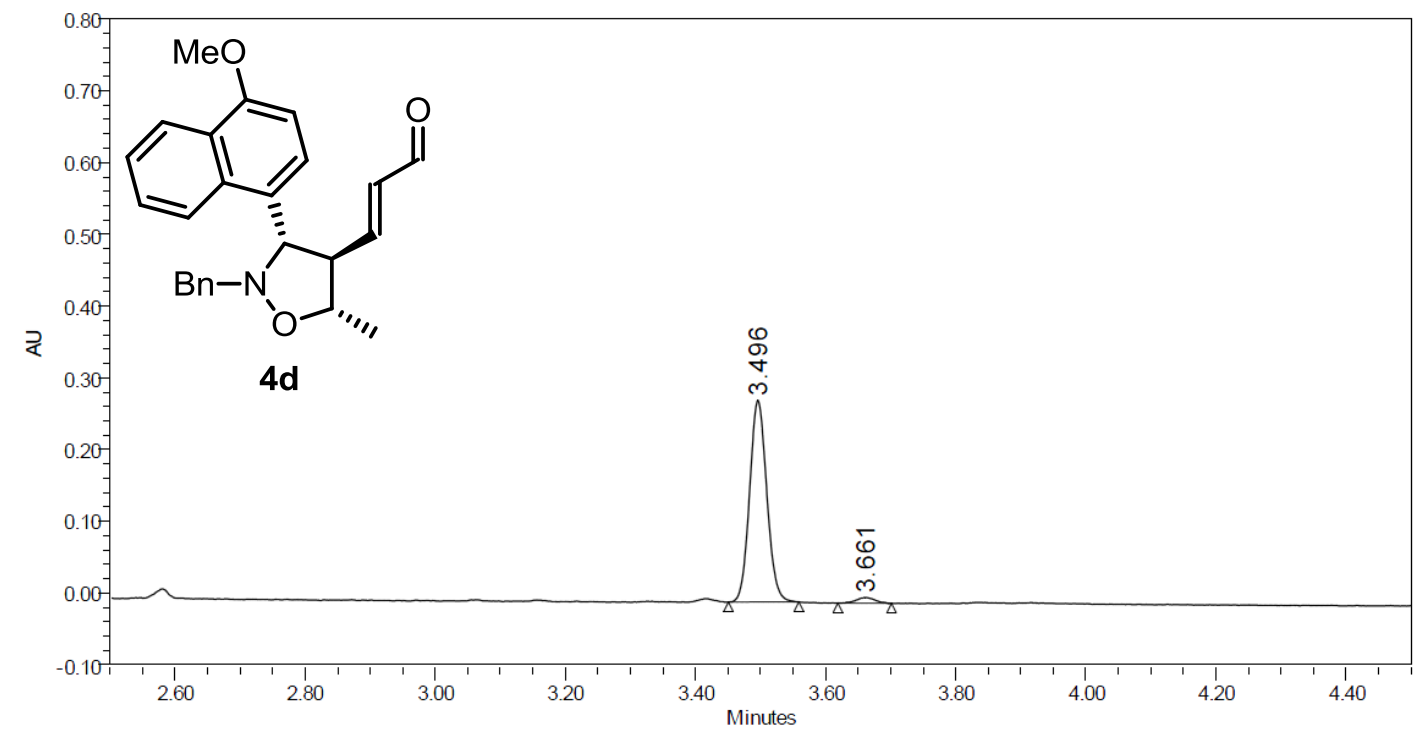

\begin{tabular}{|l|r|r|}
\hline & $\begin{array}{c}\text { Retention Time } \\
(\mathrm{min})\end{array}$ & $\%$ Area \\
\hline 1 & 3.496 & 97.02 \\
\hline 2 & 3.661 & 2.98 \\
\hline
\end{tabular}




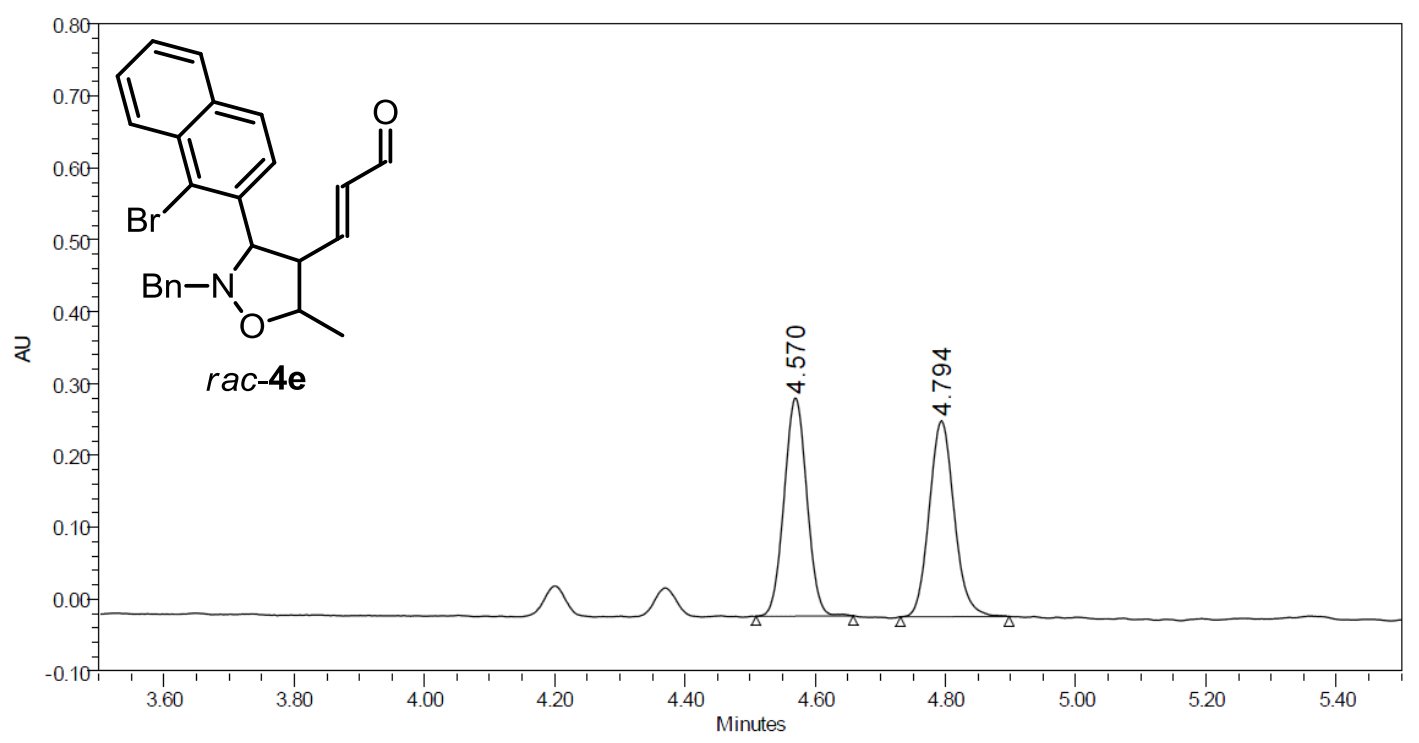

\begin{tabular}{|l|r|r|}
\hline & $\begin{array}{c}\text { Retention Time } \\
\text { (min) }\end{array}$ & $\%$ Area \\
\hline 1 & 4.570 & 50.31 \\
\hline 2 & 4.794 & 49.69 \\
\hline
\end{tabular}

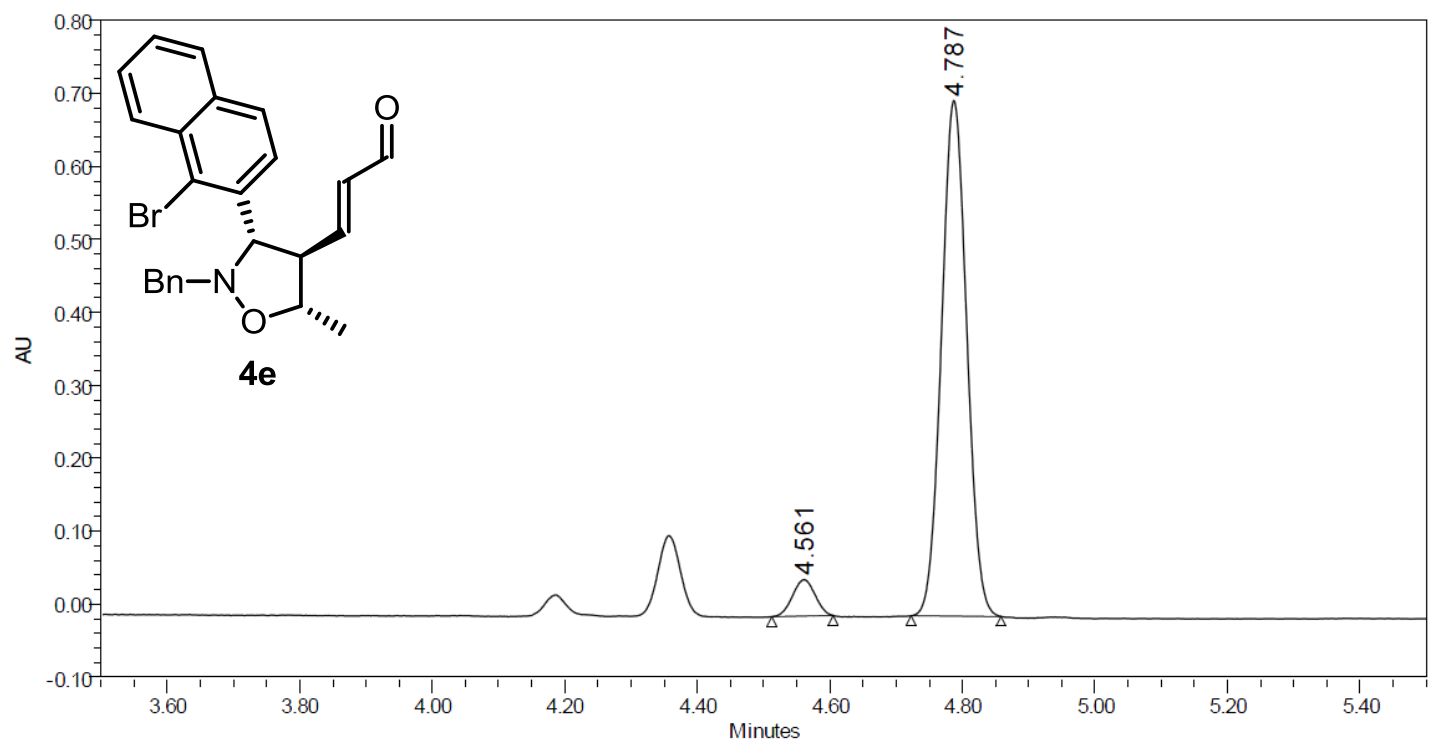

\begin{tabular}{|r|r|r|}
\hline & $\begin{array}{c}\text { Retention Time } \\
\text { (min) }\end{array}$ & \% Area \\
\hline 1 & 4.561 & 5.93 \\
\hline 2 & 4.787 & 94.07 \\
\hline
\end{tabular}




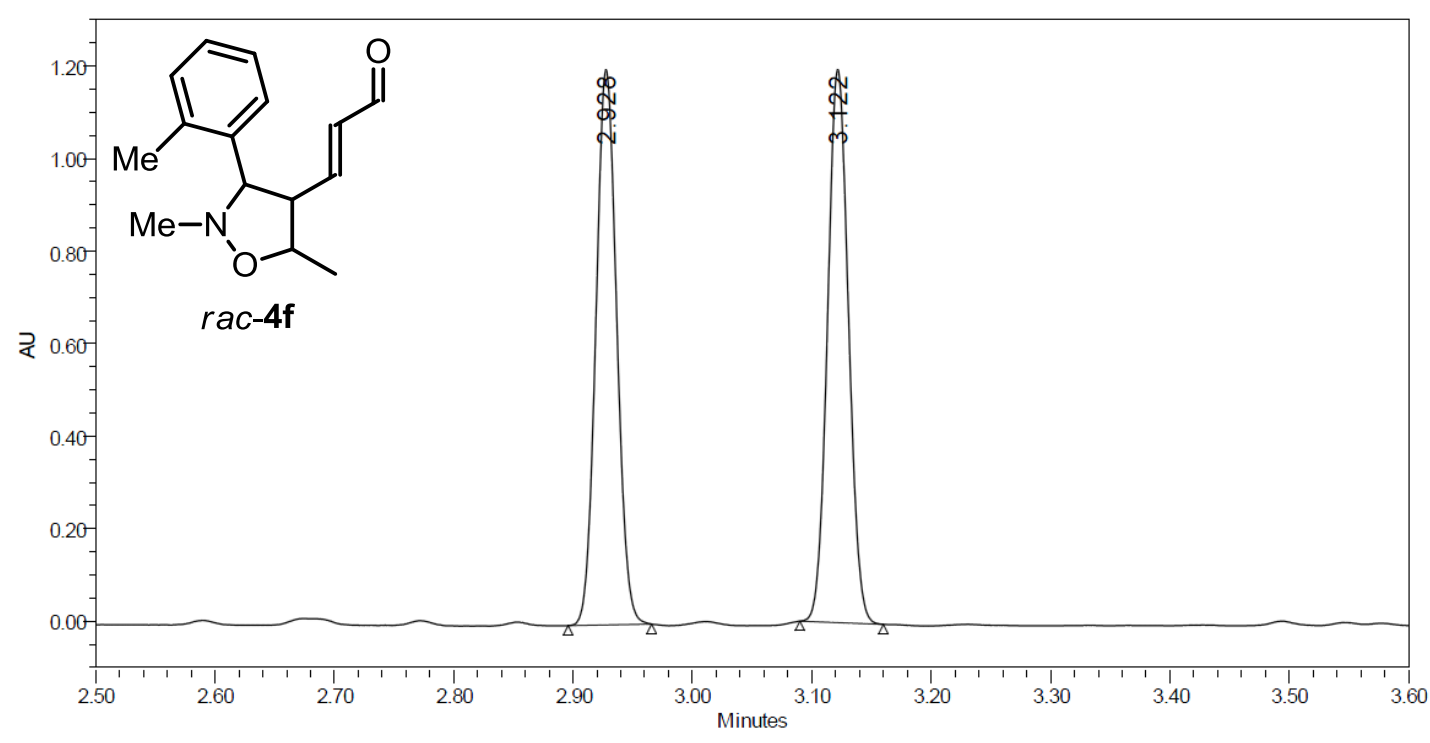

\begin{tabular}{|l|r|r|}
\hline & $\begin{array}{c}\text { Retention Time } \\
\text { (min) }\end{array}$ & \% Area \\
\hline 1 & 2.928 & 49.90 \\
\hline 2 & 3.122 & 50.10 \\
\hline
\end{tabular}

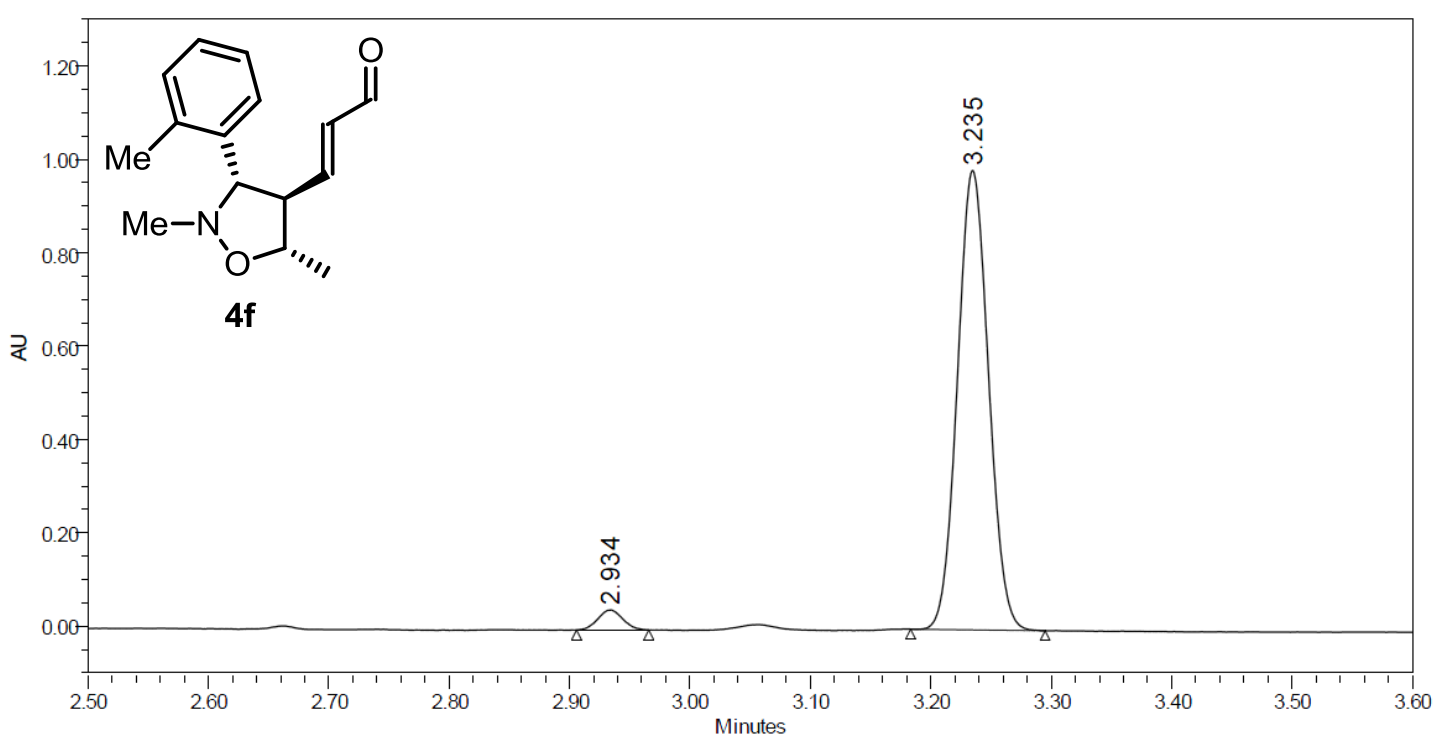

\begin{tabular}{|l|r|r|}
\hline & $\begin{array}{c}\text { Retention Time } \\
(\mathrm{min})\end{array}$ & \% Area \\
\hline 1 & 2.934 & 3.34 \\
\hline 2 & 3.235 & 96.66 \\
\hline
\end{tabular}



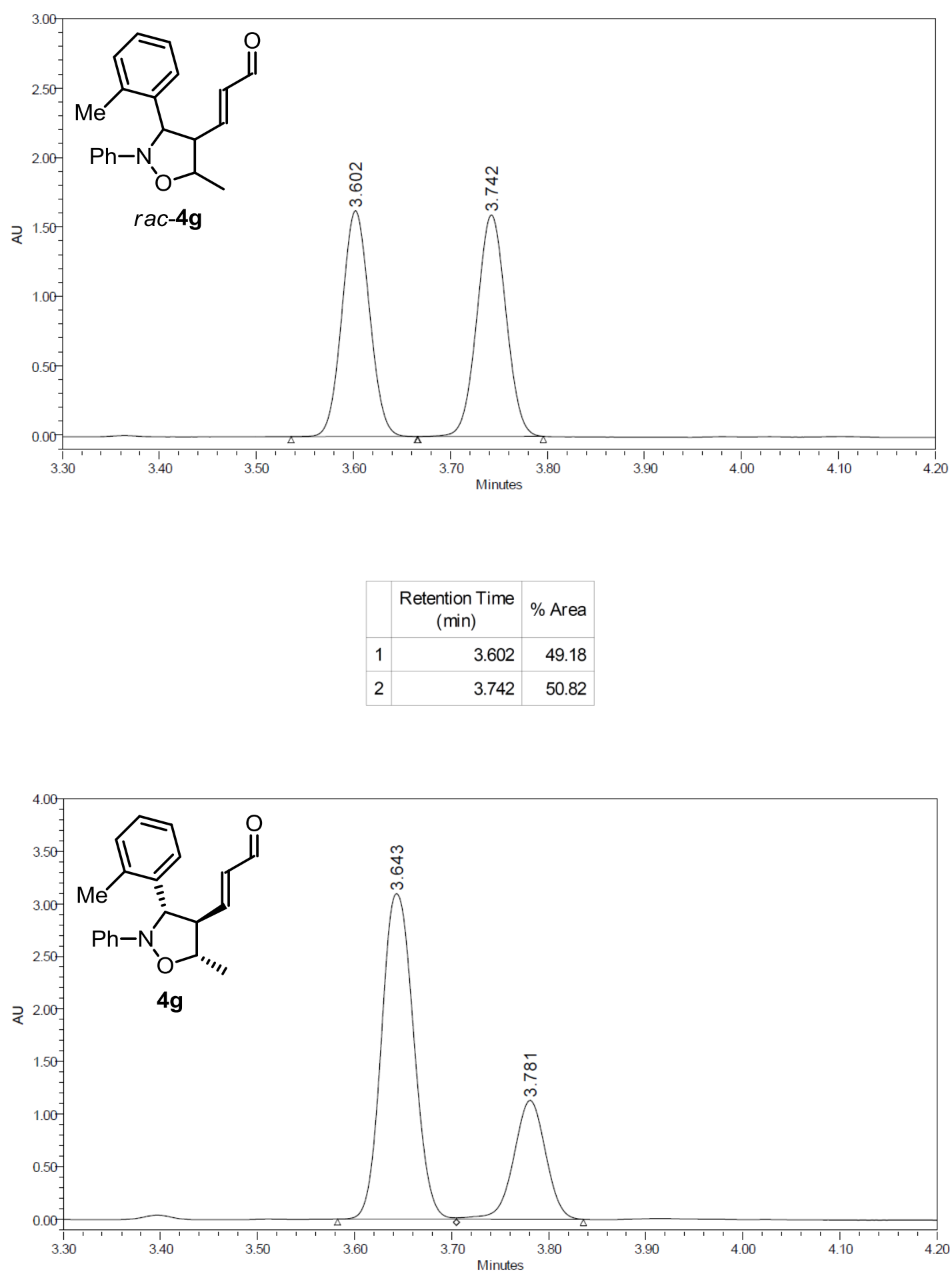

\begin{tabular}{|r|r|r|}
\hline & $\begin{array}{c}\text { Retention Time } \\
\text { (min) }\end{array}$ & \% Area \\
\hline 1 & 3.643 & 73.31 \\
\hline 2 & 3.781 & 26.69 \\
\hline
\end{tabular}




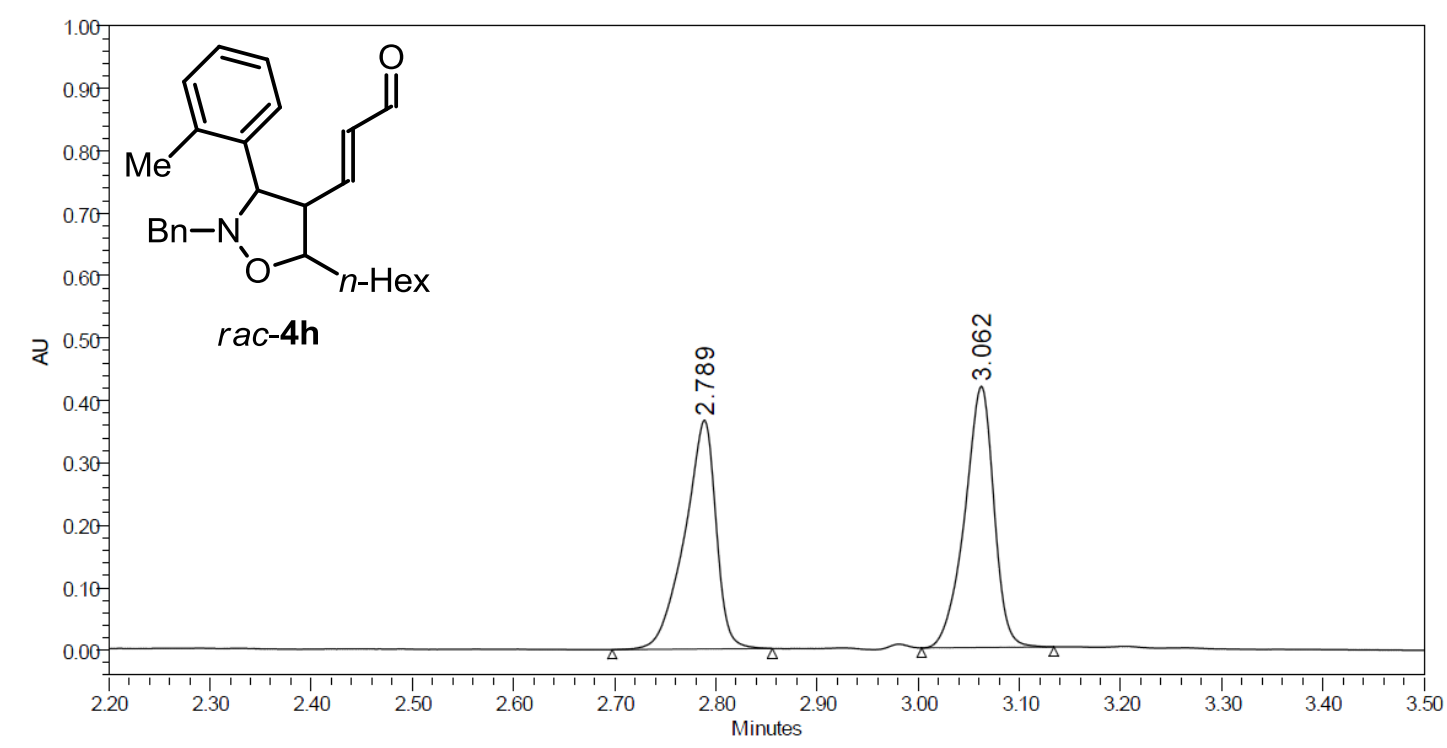

\begin{tabular}{|l|r|r|}
\hline & $\begin{array}{c}\text { Retention Time } \\
\text { (min) }\end{array}$ & \% Area \\
\hline 1 & 2.789 & 48.51 \\
\hline 2 & 3.062 & 51.49 \\
\hline
\end{tabular}

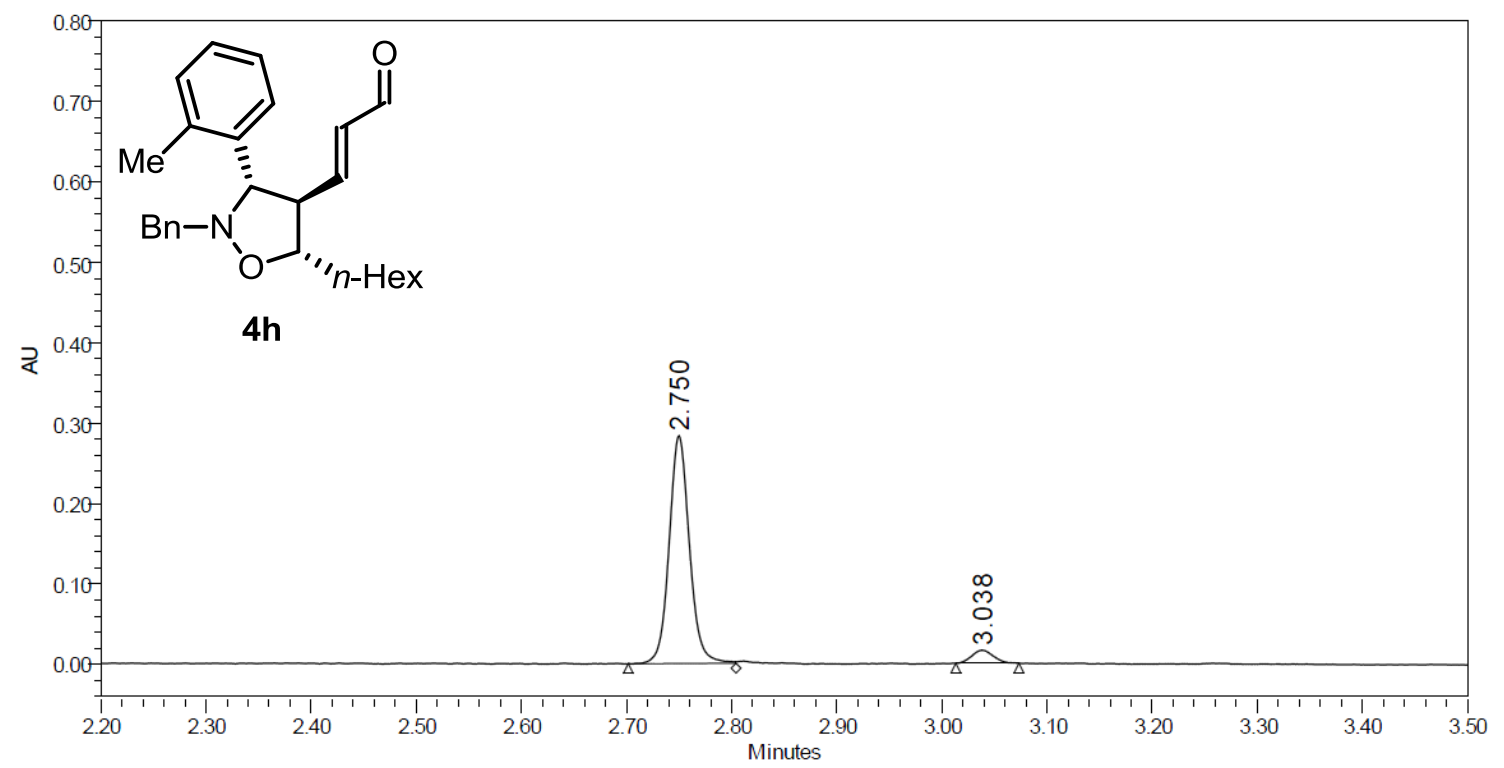

\begin{tabular}{|l|r|r|}
\hline & $\begin{array}{c}\text { Retention Time } \\
(\mathrm{min})\end{array}$ & $\%$ Area \\
\hline 1 & 2.750 & 94.36 \\
\hline 2 & 3.038 & 5.64 \\
\hline
\end{tabular}




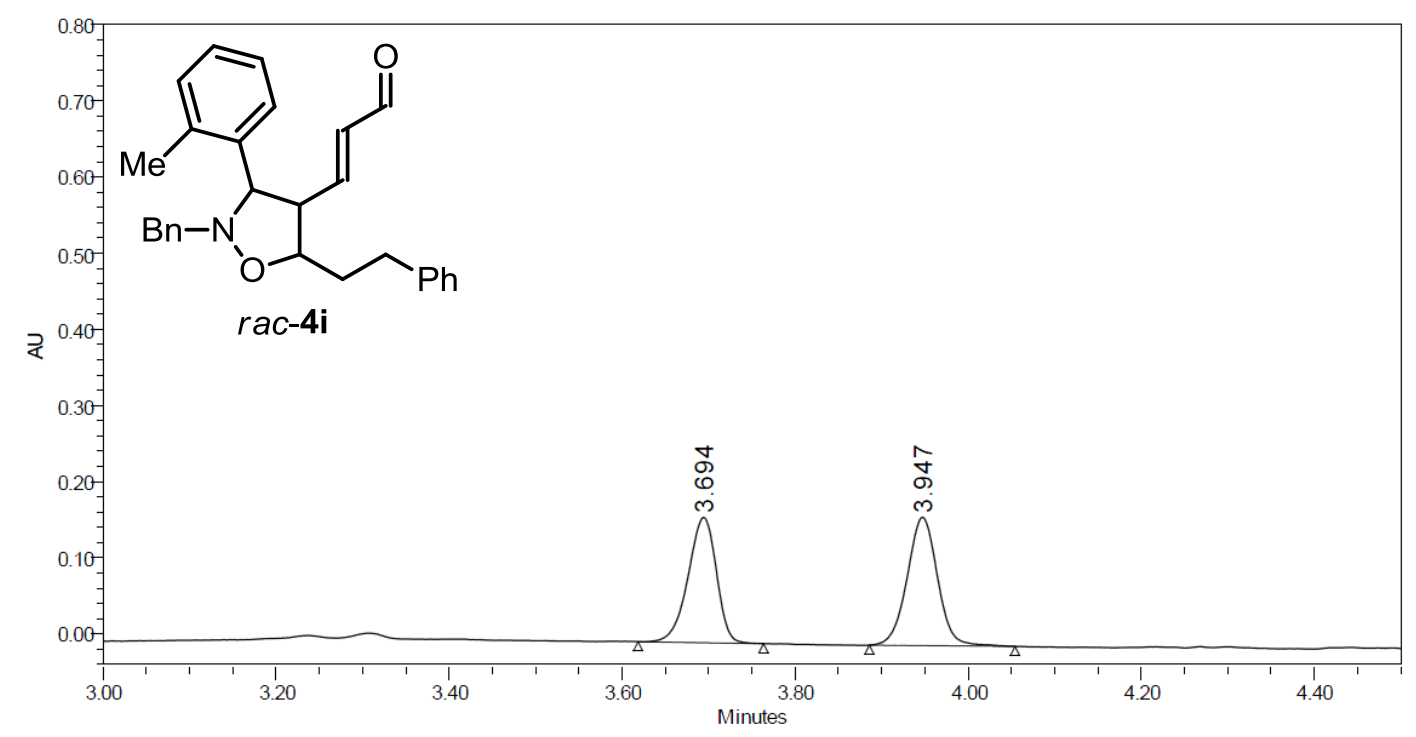

\begin{tabular}{|l|r|r|}
\hline & $\begin{array}{c}\text { Retention Time } \\
(\mathrm{min})\end{array}$ & \% Area \\
\hline 1 & 3.694 & 47.71 \\
\hline 2 & 3.947 & 52.29 \\
\hline
\end{tabular}

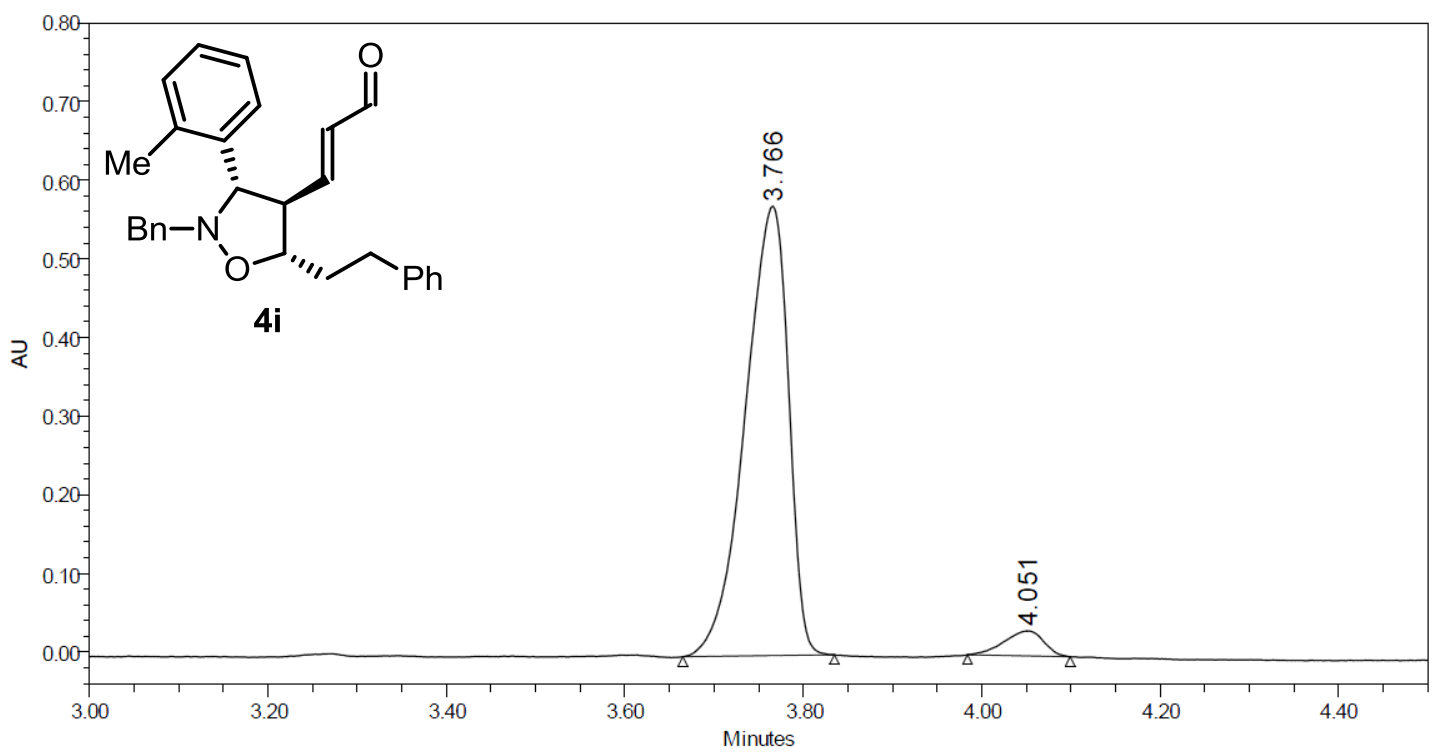

\begin{tabular}{|l|r|r|}
\hline & $\begin{array}{c}\text { Retention Time } \\
\text { (min) }\end{array}$ & \% Area \\
\hline 1 & 3.766 & 95.14 \\
\hline 2 & 4.051 & 4.86 \\
\hline
\end{tabular}




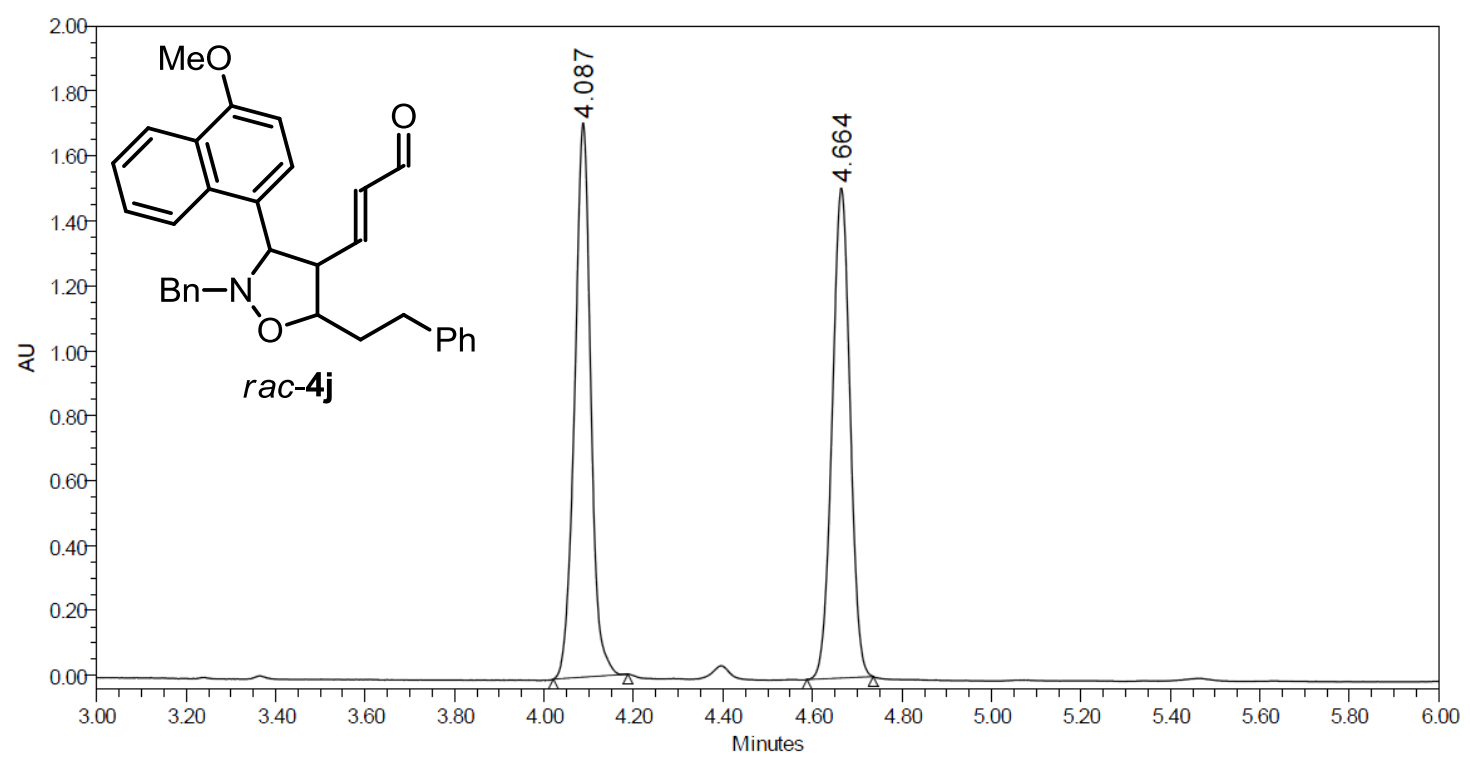

\begin{tabular}{|l|r|r|}
\hline & $\begin{array}{c}\text { Retention Time } \\
(\mathrm{min})\end{array}$ & \% Area \\
\hline 1 & 4.087 & 50.44 \\
\hline 2 & 4.664 & 49.56 \\
\hline
\end{tabular}

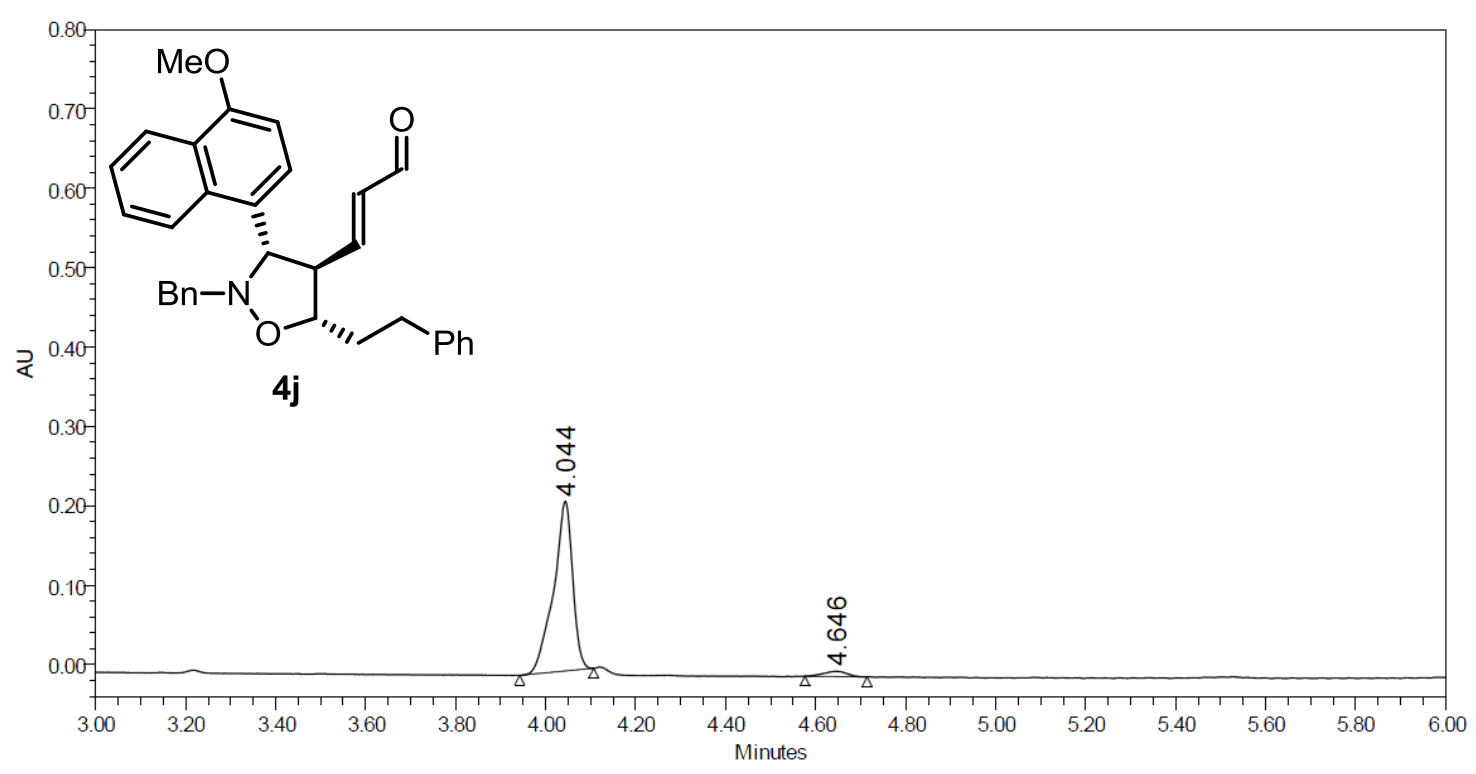

\begin{tabular}{|r|r|r|}
\hline & $\begin{array}{c}\text { Retention Time } \\
\text { (min) }\end{array}$ & $\%$ Area \\
\hline 1 & 4.044 & 96.43 \\
\hline 2 & 4.646 & 3.57 \\
\hline
\end{tabular}




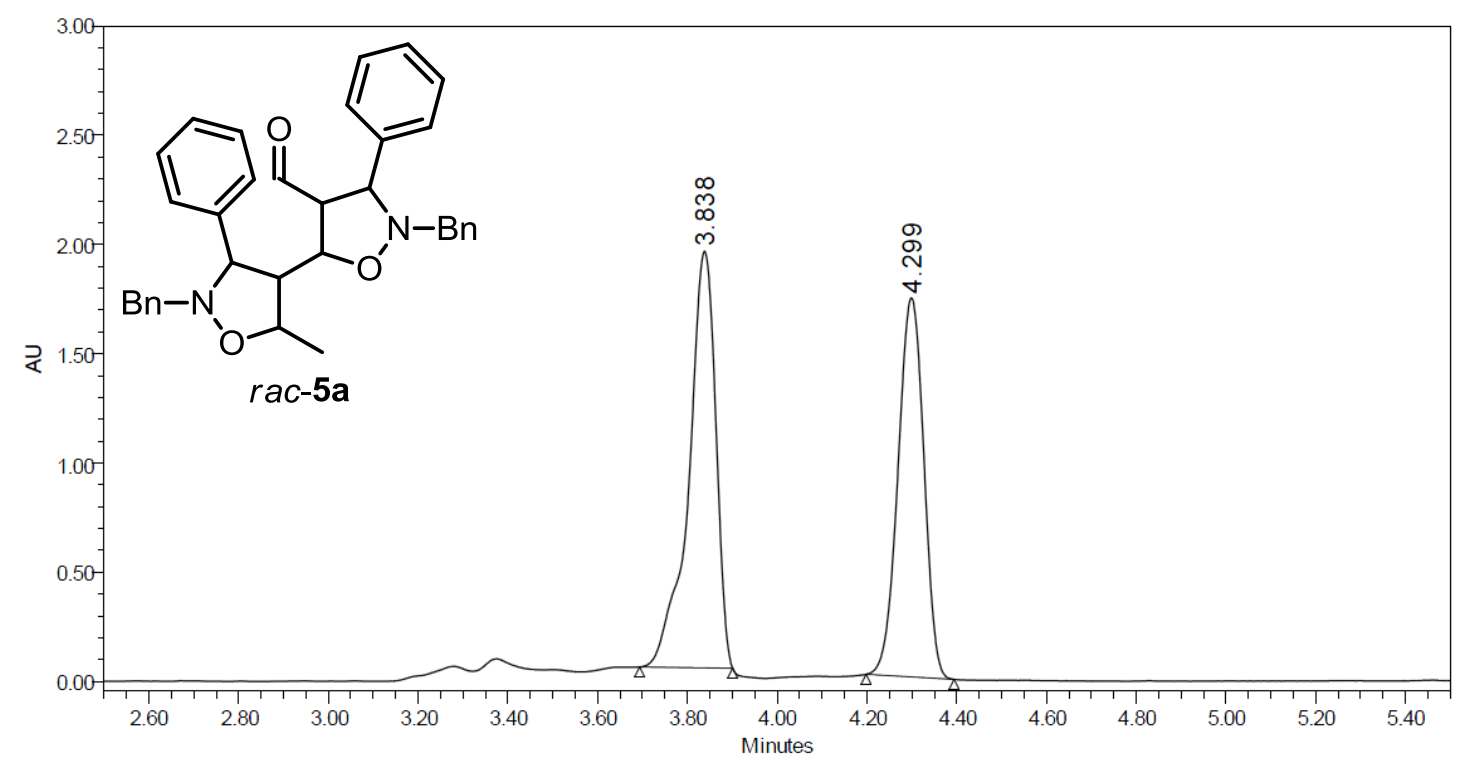

\begin{tabular}{|l|r|r|}
\hline & $\begin{array}{c}\text { Retention Time } \\
\text { (min) }\end{array}$ & \% Area \\
\hline 1 & 3.838 & 53.53 \\
\hline 2 & 4.299 & 46.47 \\
\hline
\end{tabular}

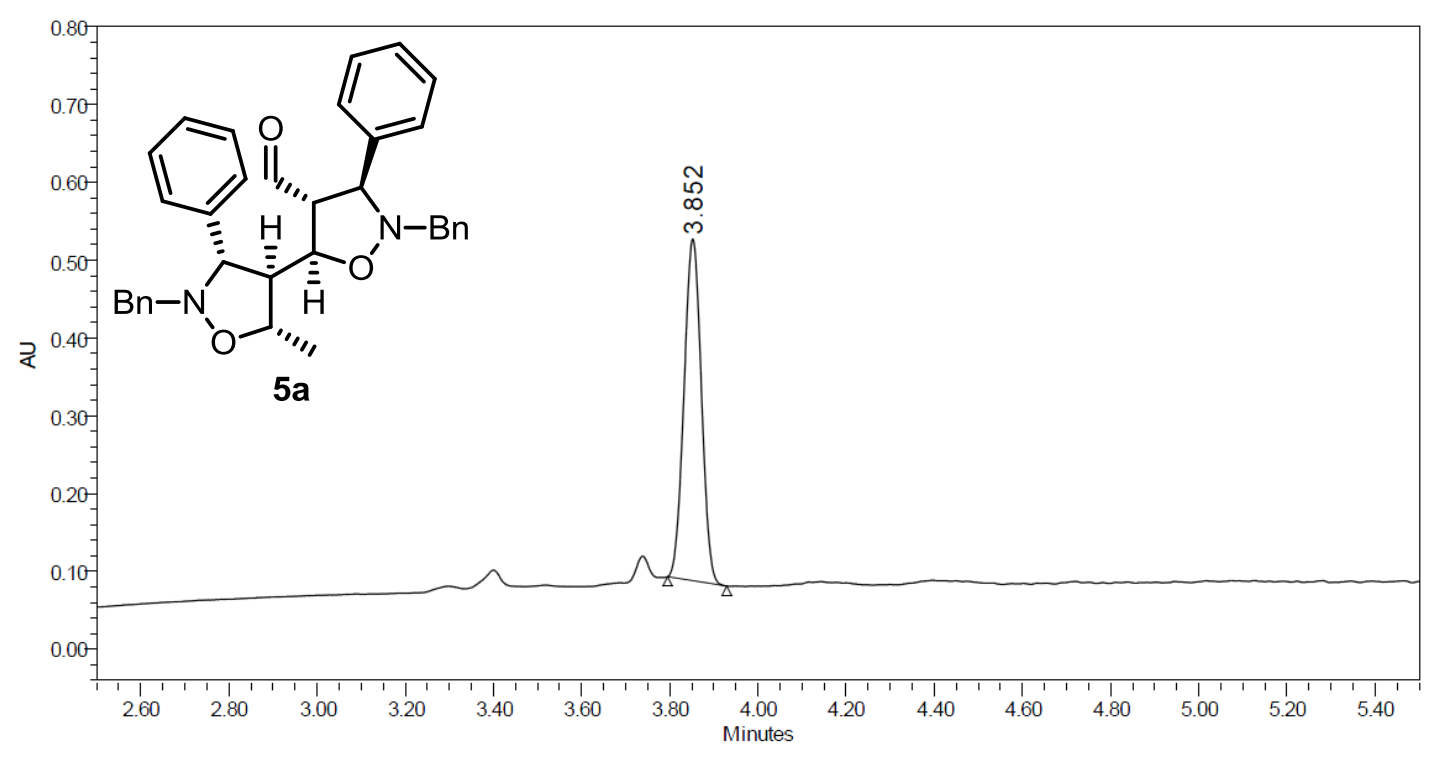

\begin{tabular}{|l|r|r|}
\hline & $\begin{array}{c}\text { Retention Time } \\
(\mathrm{min})\end{array}$ & $\%$ Area \\
\hline 1 & 3.852 & 100.00 \\
\hline
\end{tabular}




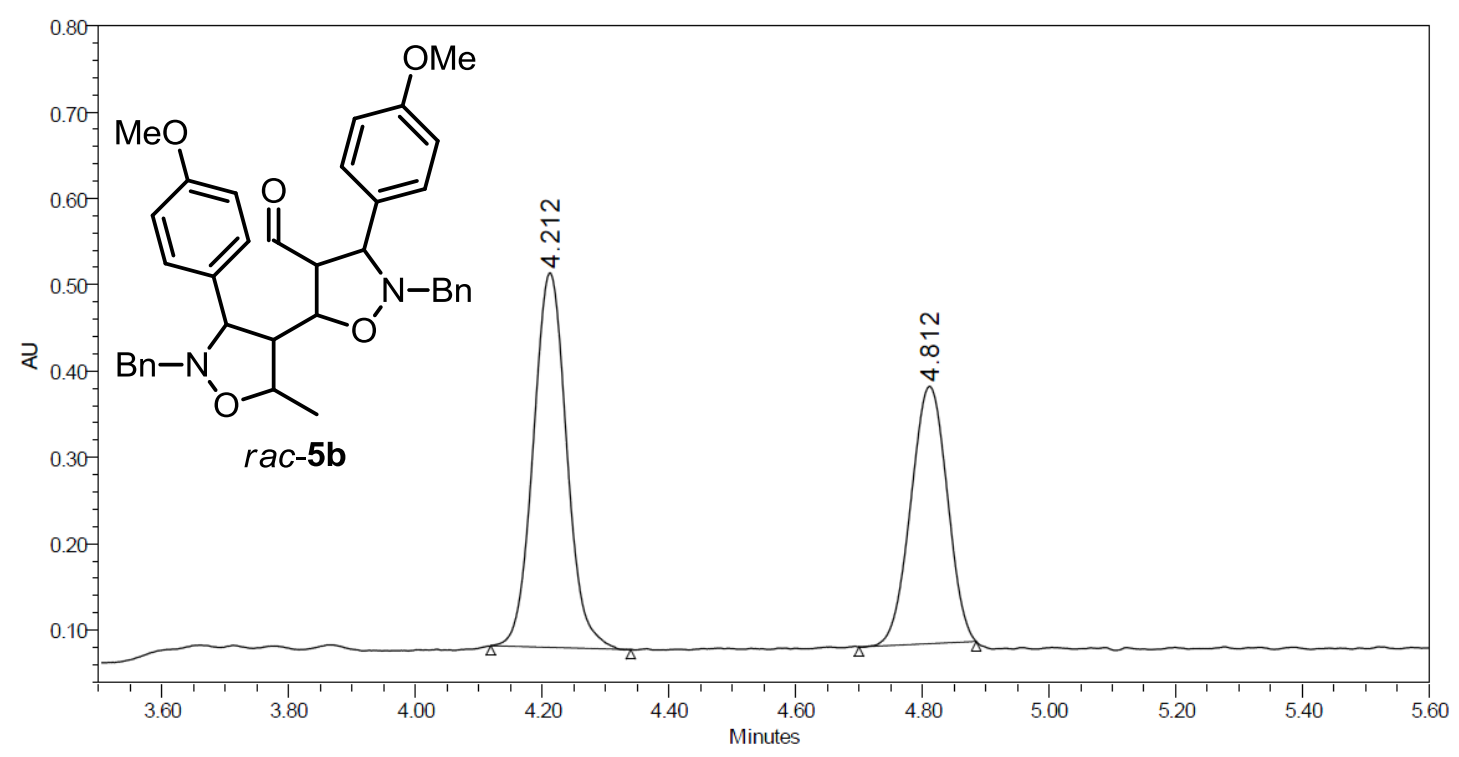

\begin{tabular}{|l|r|r|}
\hline & $\begin{array}{c}\text { Retention Time } \\
(\mathrm{min})\end{array}$ & $\%$ Area \\
\hline 1 & 4.212 & 57.19 \\
\hline 2 & 4.812 & 42.81 \\
\hline
\end{tabular}

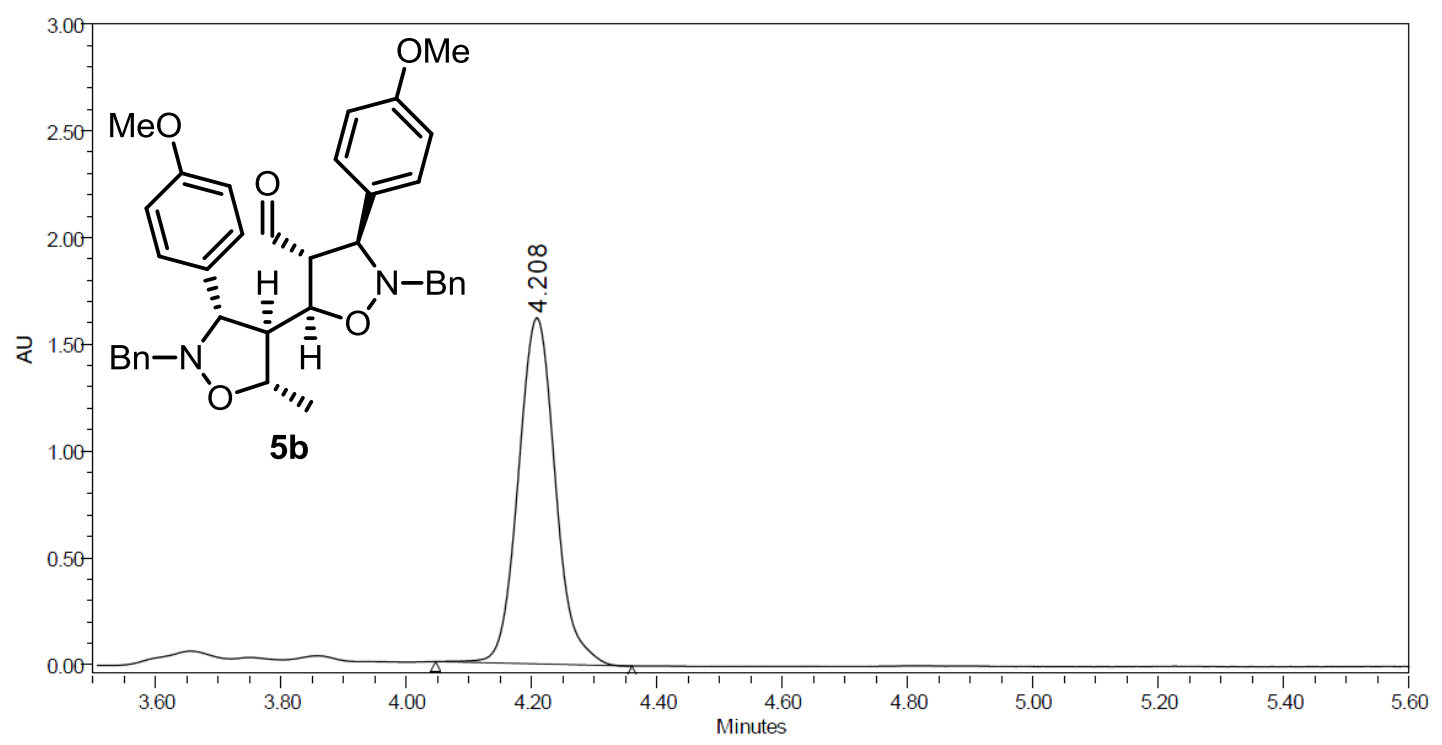

\begin{tabular}{|c|r|c|}
\hline & $\begin{array}{c}\text { Retention Time } \\
(\mathrm{min})\end{array}$ & $\%$ Area \\
\hline 1 & 4.208 & 100.00 \\
\hline
\end{tabular}




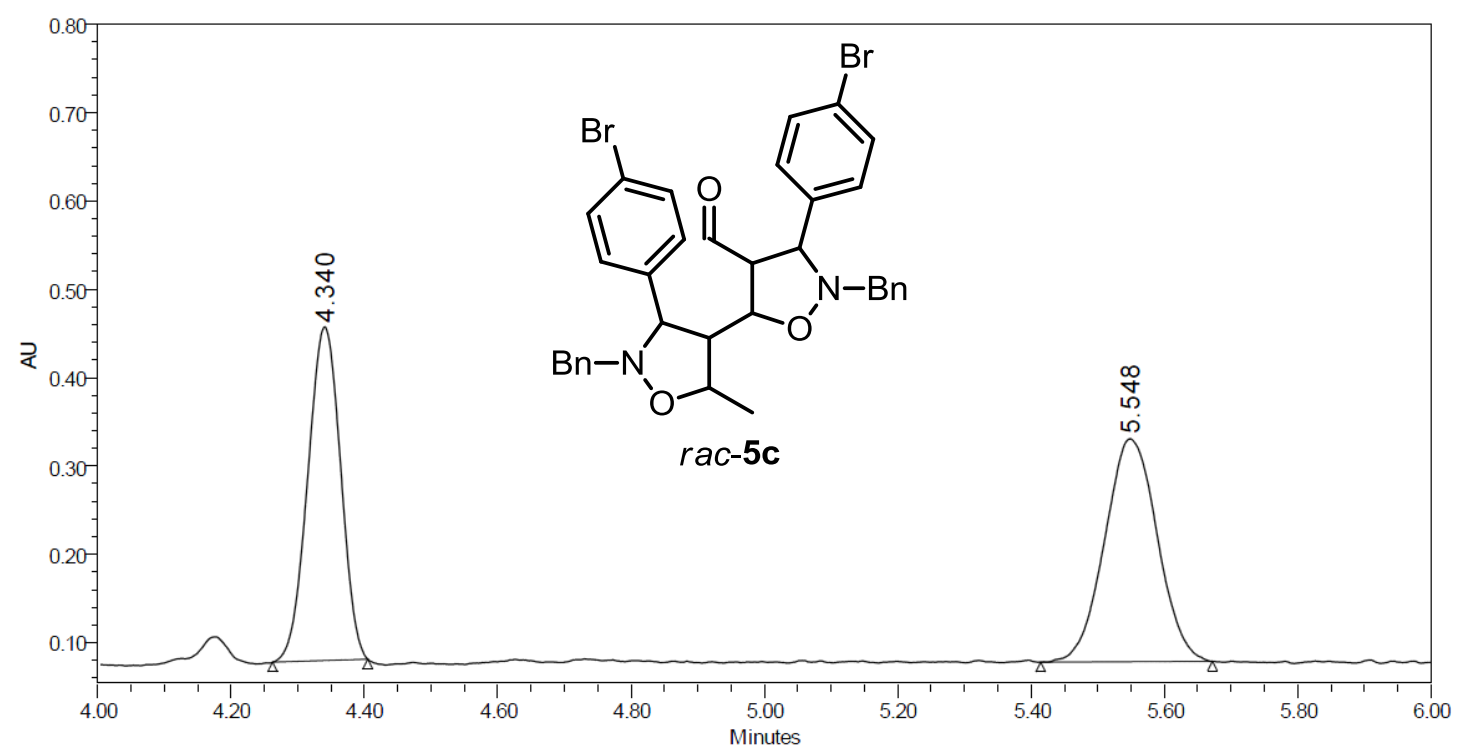

\begin{tabular}{|r|r|r|}
\hline & $\begin{array}{c}\text { Retention Time } \\
(\mathrm{min})\end{array}$ & \% Area \\
\hline 1 & 4.340 & 48.26 \\
\hline 2 & 5.548 & 51.74 \\
\hline
\end{tabular}

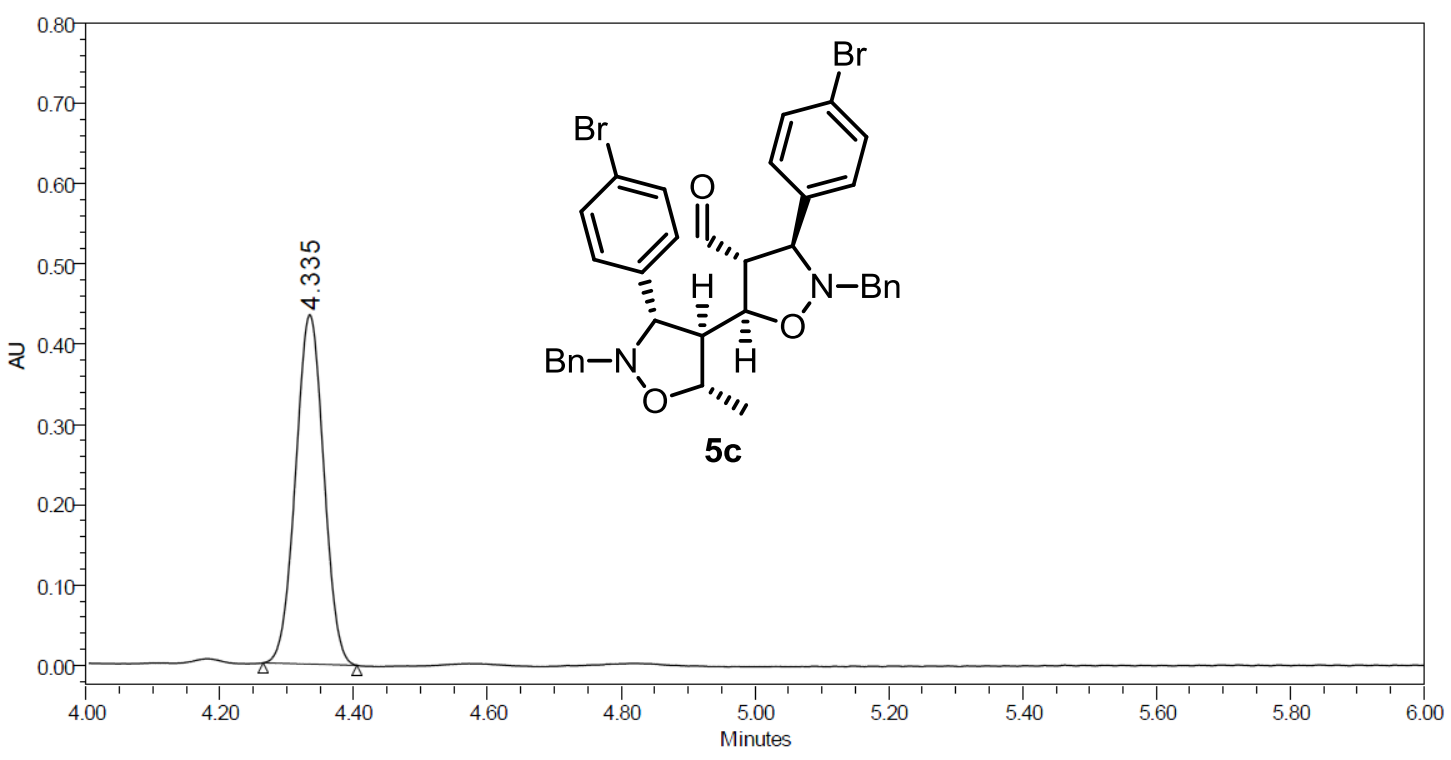

\begin{tabular}{|l|r|r|}
\hline & $\begin{array}{c}\text { Retention Time } \\
(\mathrm{min})\end{array}$ & $\%$ Area \\
\hline 1 & 4.335 & 100.00 \\
\hline
\end{tabular}




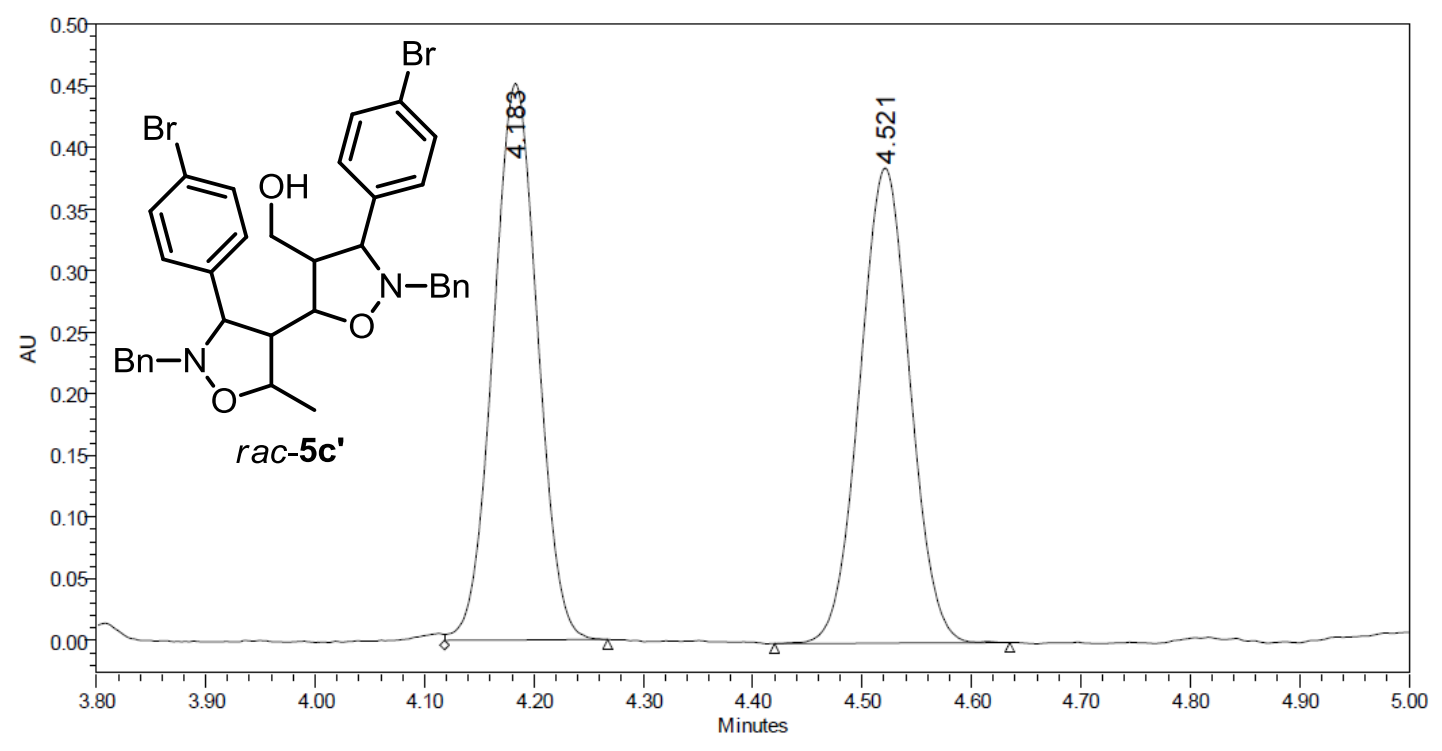

\begin{tabular}{|l|r|r|}
\hline & $\begin{array}{c}\text { Retention Time } \\
\text { (min) }\end{array}$ & \% Area \\
\hline 1 & 4.183 & 50.81 \\
\hline 2 & 4.521 & 49.19 \\
\hline
\end{tabular}

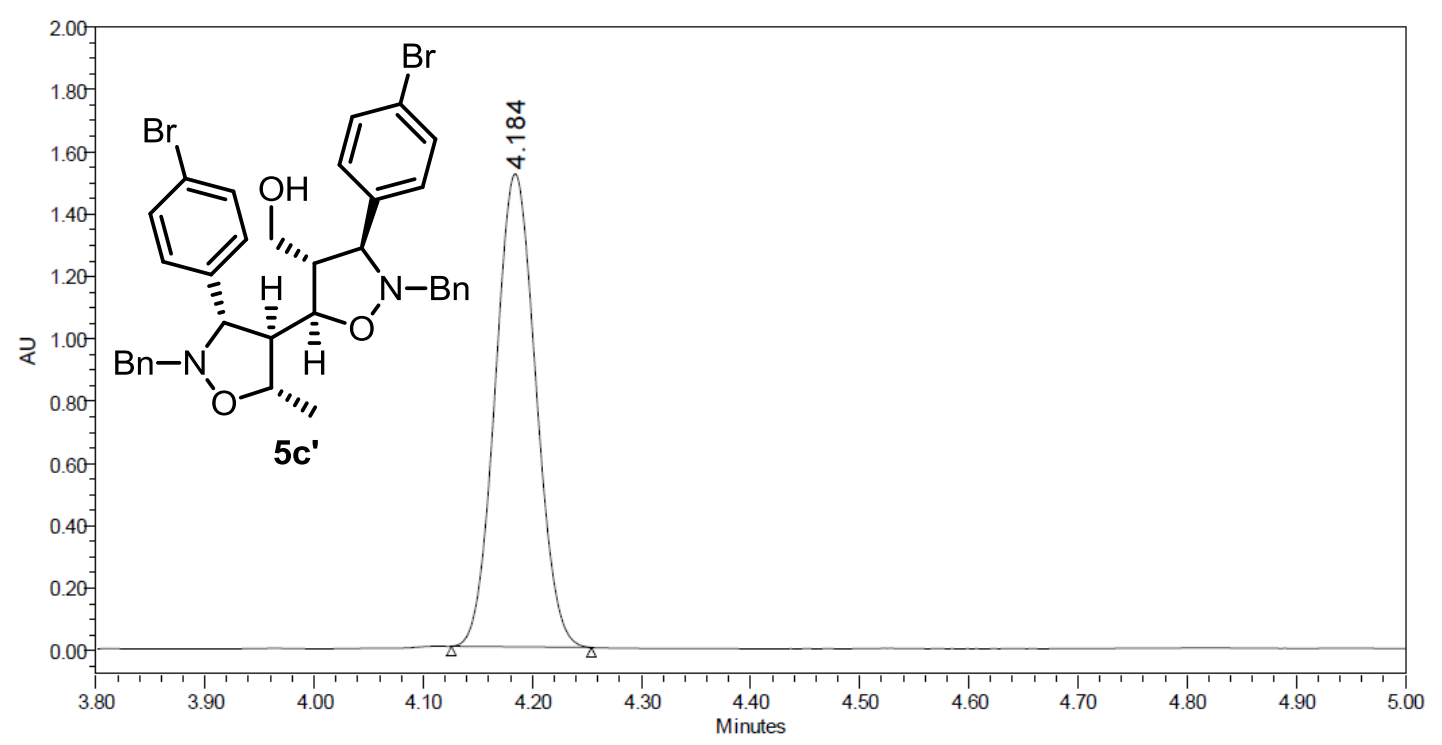

\begin{tabular}{|c|r|r|}
\hline & $\begin{array}{c}\text { Retention Time } \\
\text { (min) }\end{array}$ & $\%$ Area \\
\hline 1 & 4.184 & 100.00 \\
\hline
\end{tabular}




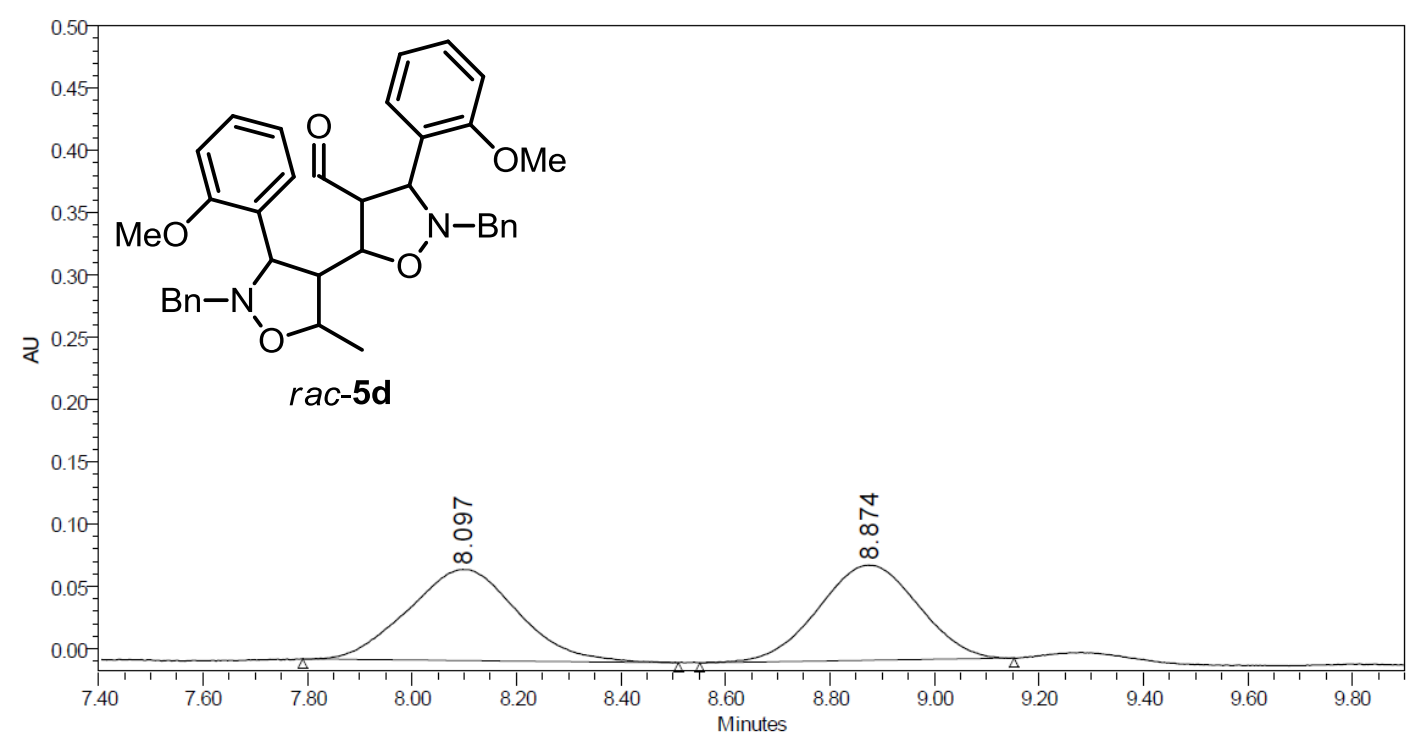

\begin{tabular}{|l|r|r|}
\hline & $\begin{array}{c}\text { Retention Time } \\
\text { (min) }\end{array}$ & \% Area \\
\hline 1 & 8.097 & 51.27 \\
\hline 2 & 8.874 & 48.73 \\
\hline
\end{tabular}

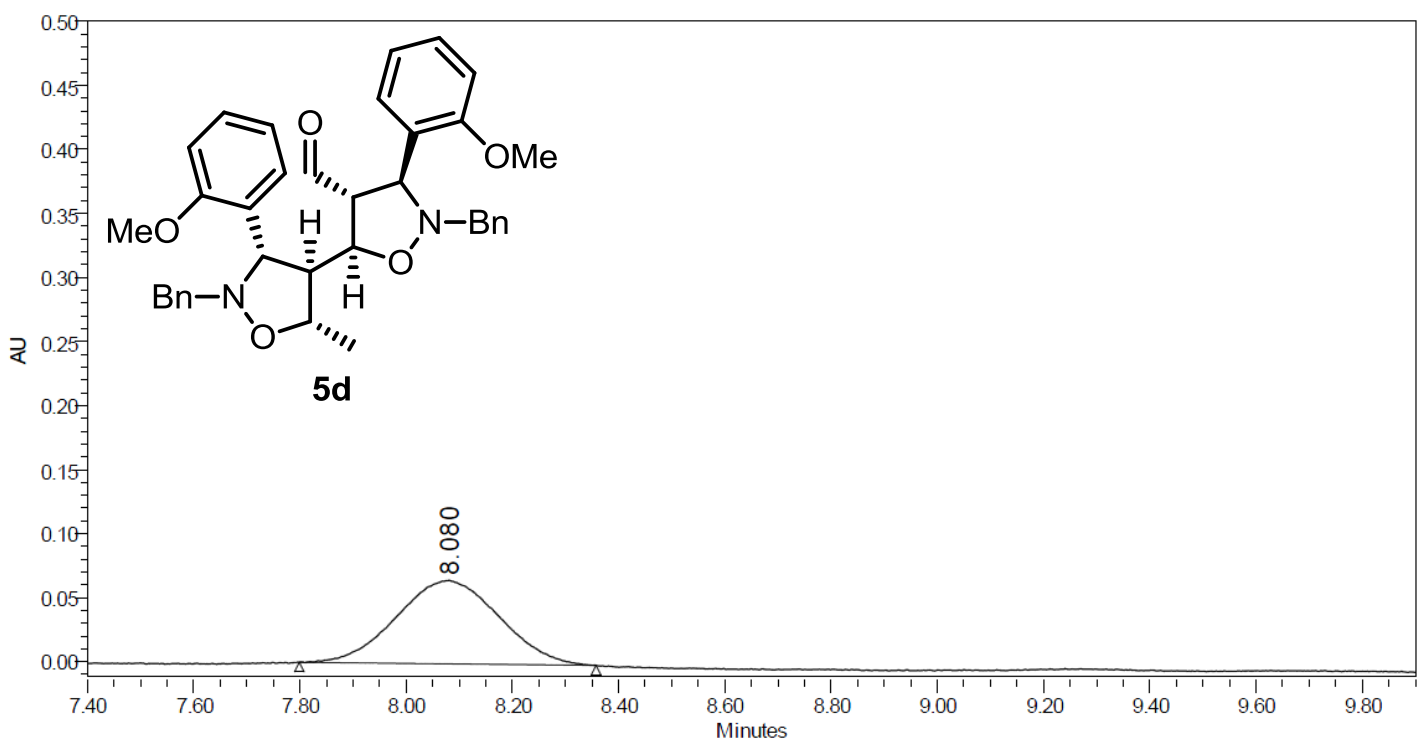

\begin{tabular}{|l|r|r|}
\hline & $\begin{array}{c}\text { Retention Time } \\
(\mathrm{min})\end{array}$ & $\%$ Area \\
\hline 1 & 8.080 & 100.00 \\
\hline
\end{tabular}




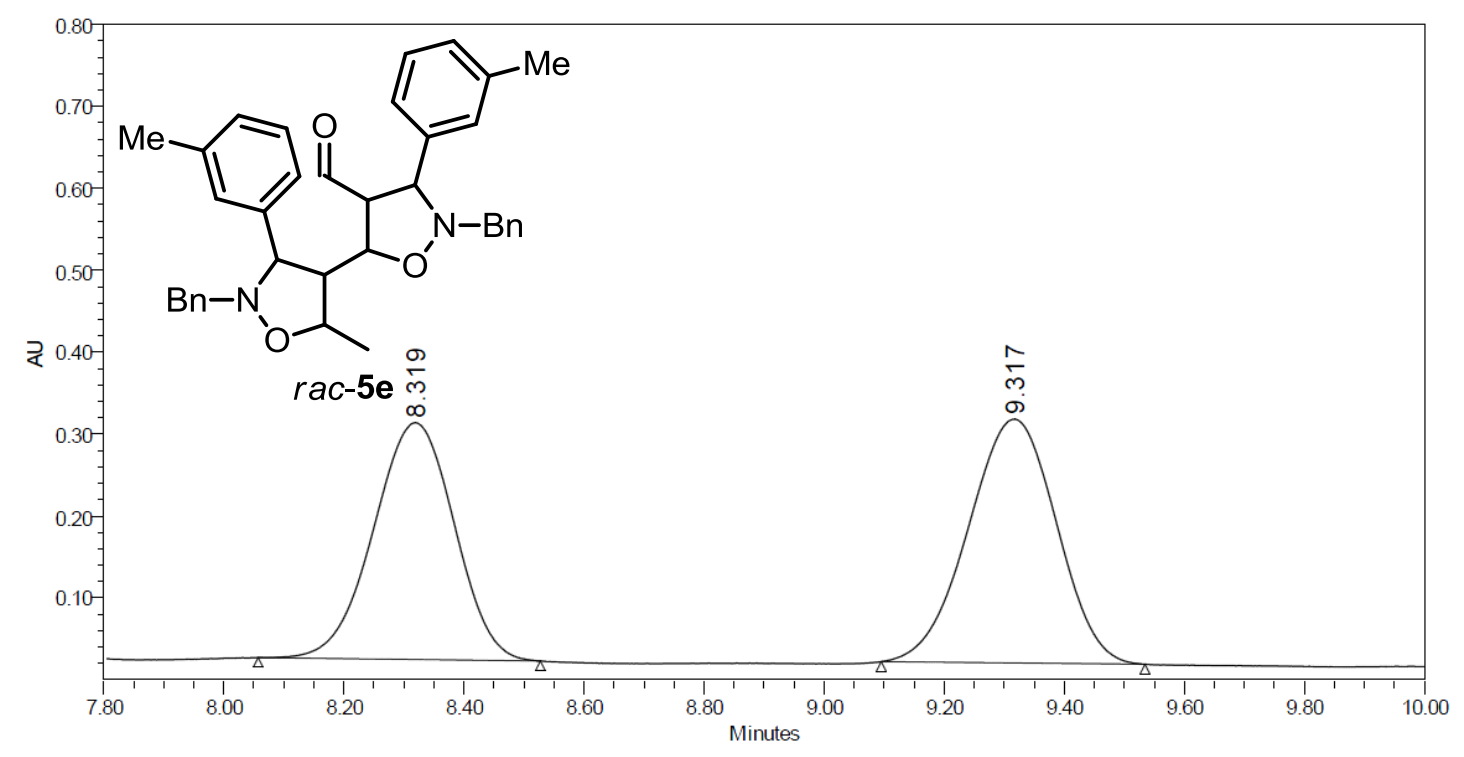

\begin{tabular}{|l|r|r|}
\hline & $\begin{array}{c}\text { Retention Time } \\
\text { (min) }\end{array}$ & $\%$ Area \\
\hline 1 & 8.319 & 47.13 \\
\hline 2 & 9.317 & 52.87 \\
\hline
\end{tabular}

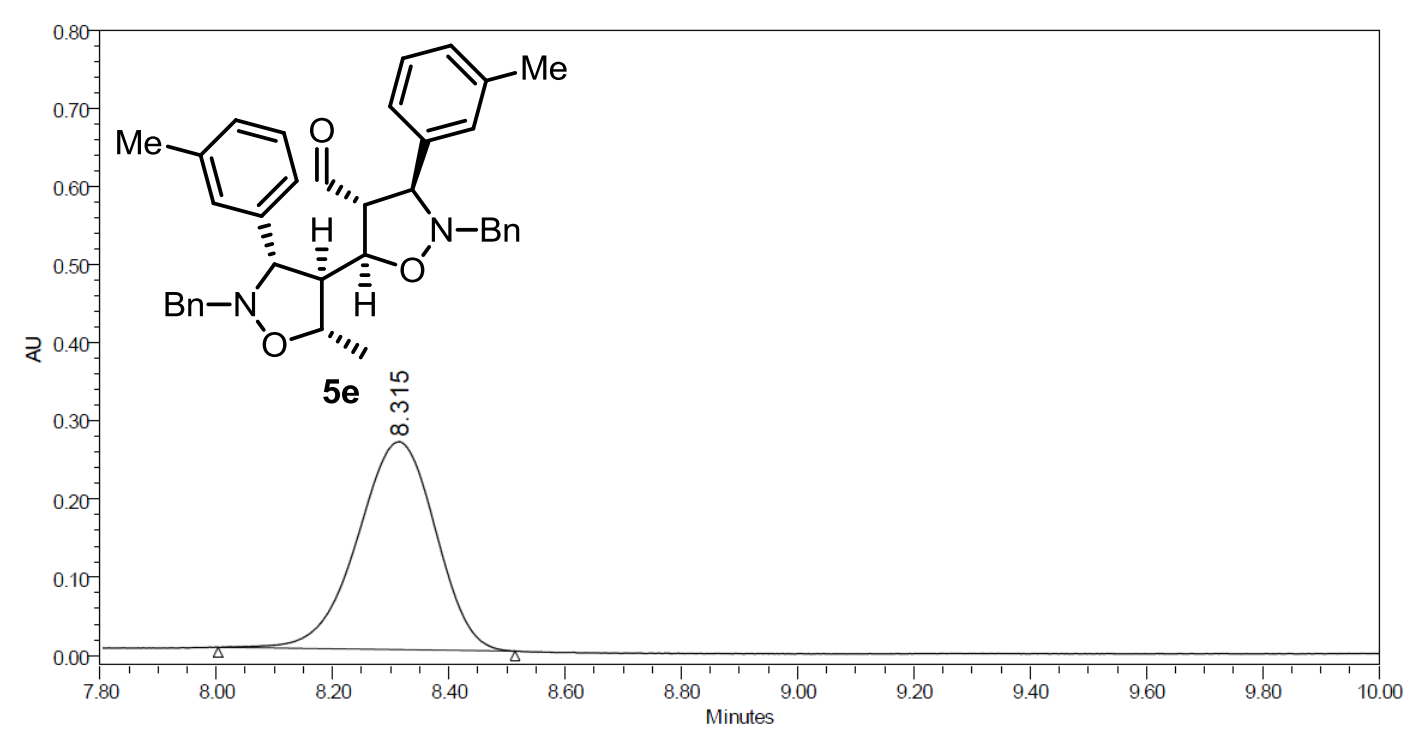

\begin{tabular}{|r|r|r|}
\hline & $\begin{array}{c}\text { Retention Time } \\
\text { (min) }\end{array}$ & $\%$ Area \\
\hline 1 & 8.315 & 100.00 \\
\hline
\end{tabular}




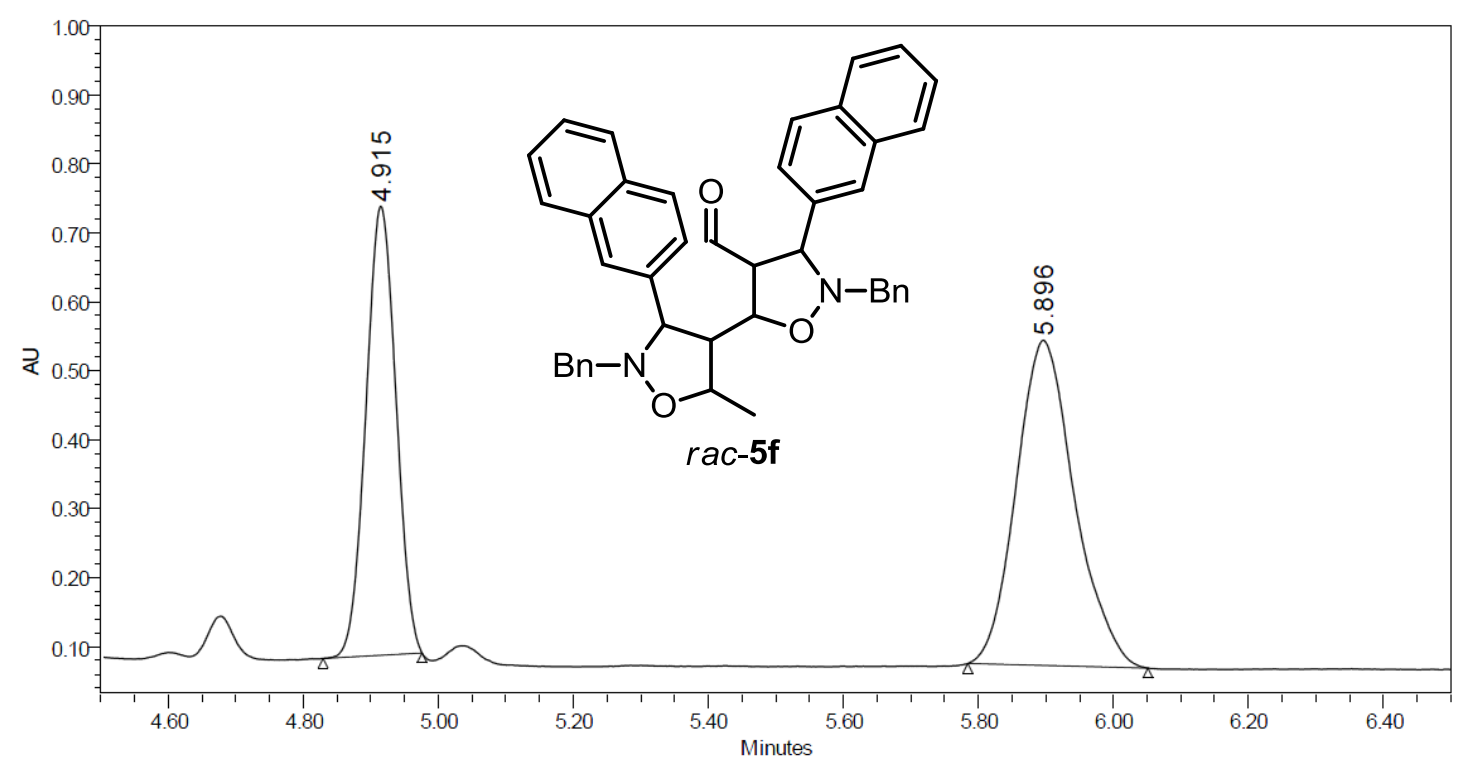

\begin{tabular}{|l|r|r|}
\hline & $\begin{array}{c}\text { Retention Time } \\
(\mathrm{min})\end{array}$ & $\%$ Area \\
\hline 1 & 4.915 & 41.66 \\
\hline 2 & 5.896 & 58.34 \\
\hline
\end{tabular}

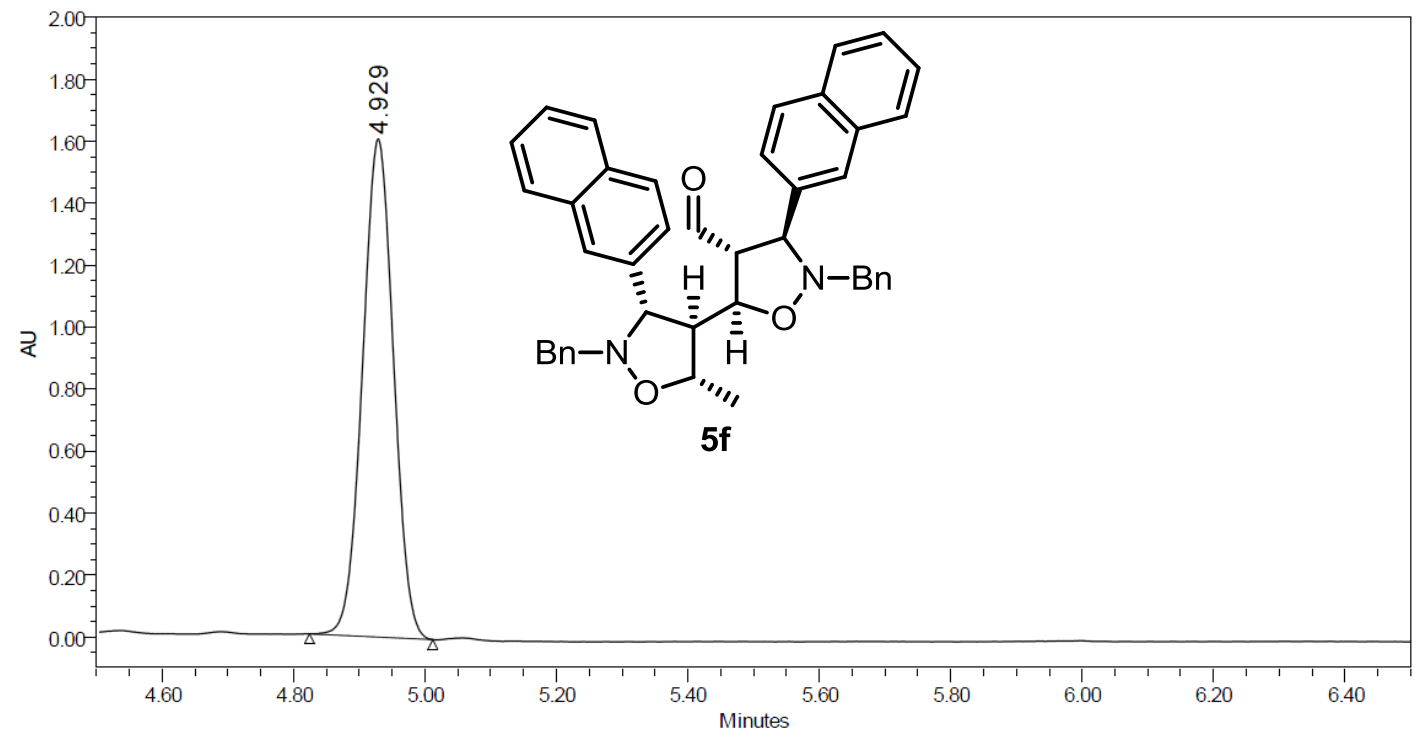

\begin{tabular}{|l|r|r|}
\hline & $\begin{array}{c}\text { Retention Time } \\
(\mathrm{min})\end{array}$ & $\%$ Area \\
\hline 1 & 4.929 & 100.00 \\
\hline
\end{tabular}



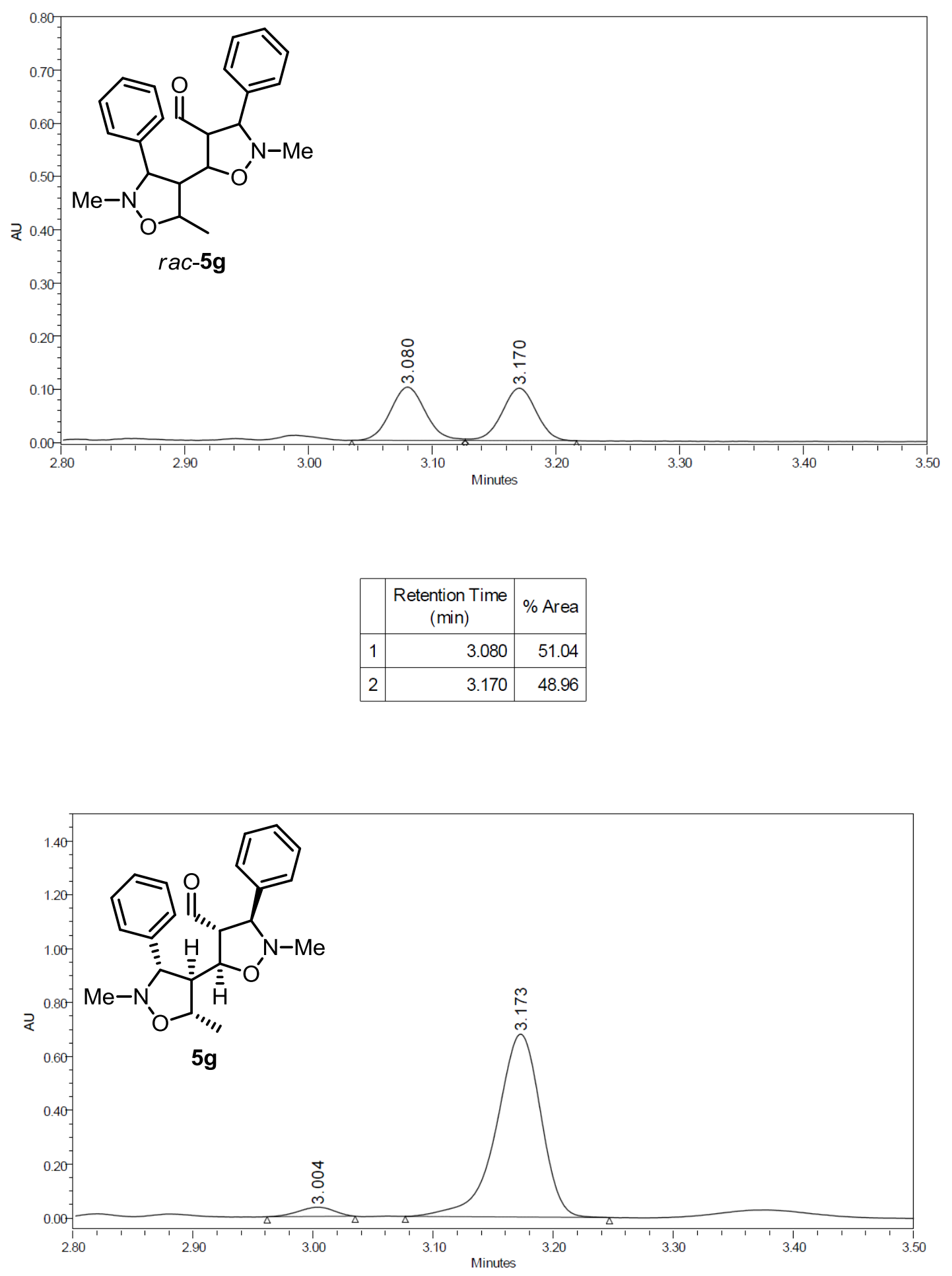

\begin{tabular}{|l|r|r|}
\hline & $\begin{array}{c}\text { Retention Time } \\
\text { (min) }\end{array}$ & \% Area \\
\hline 1 & 3.004 & 3.87 \\
\hline 2 & 3.173 & 96.13 \\
\hline
\end{tabular}




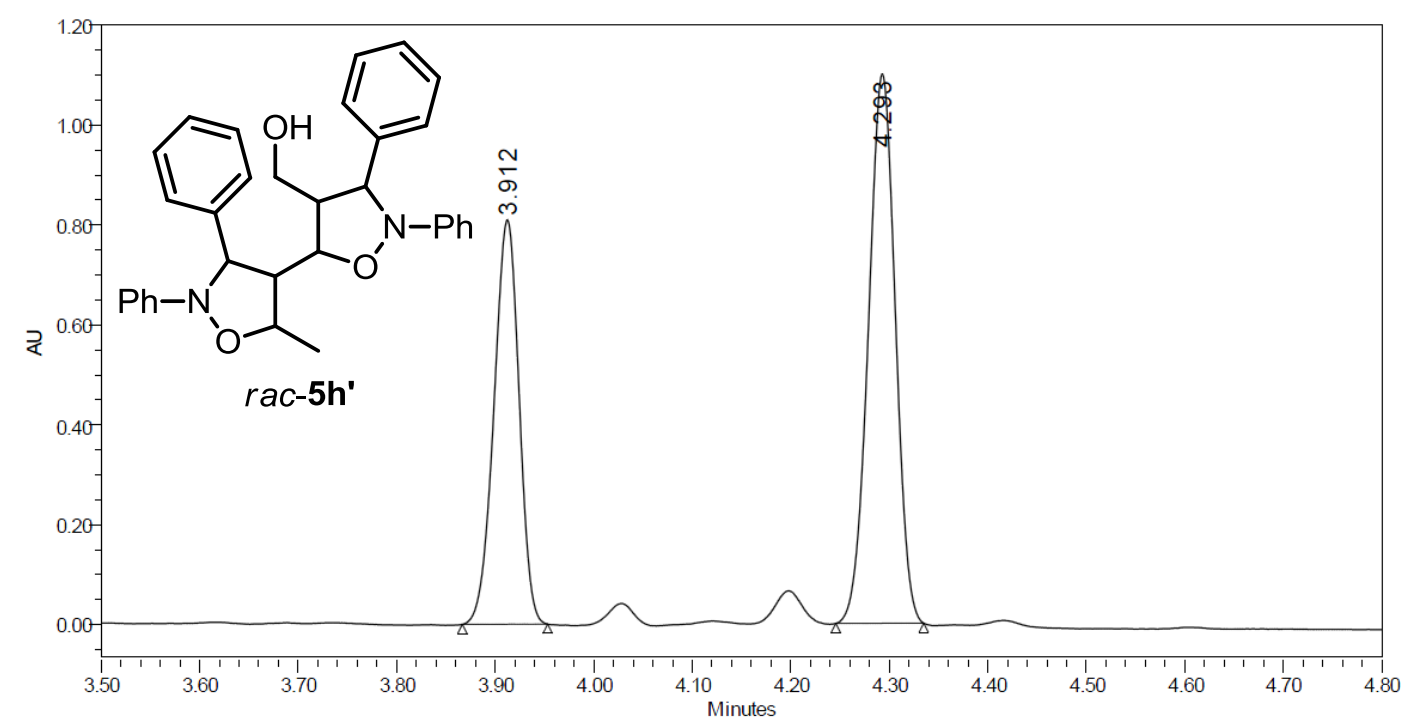

\begin{tabular}{|l|r|r|}
\hline & $\begin{array}{c}\text { Retention Time } \\
(\mathrm{min})\end{array}$ & $\%$ Area \\
\hline 1 & 3.912 & 40.76 \\
\hline 2 & 4.293 & 59.24 \\
\hline
\end{tabular}

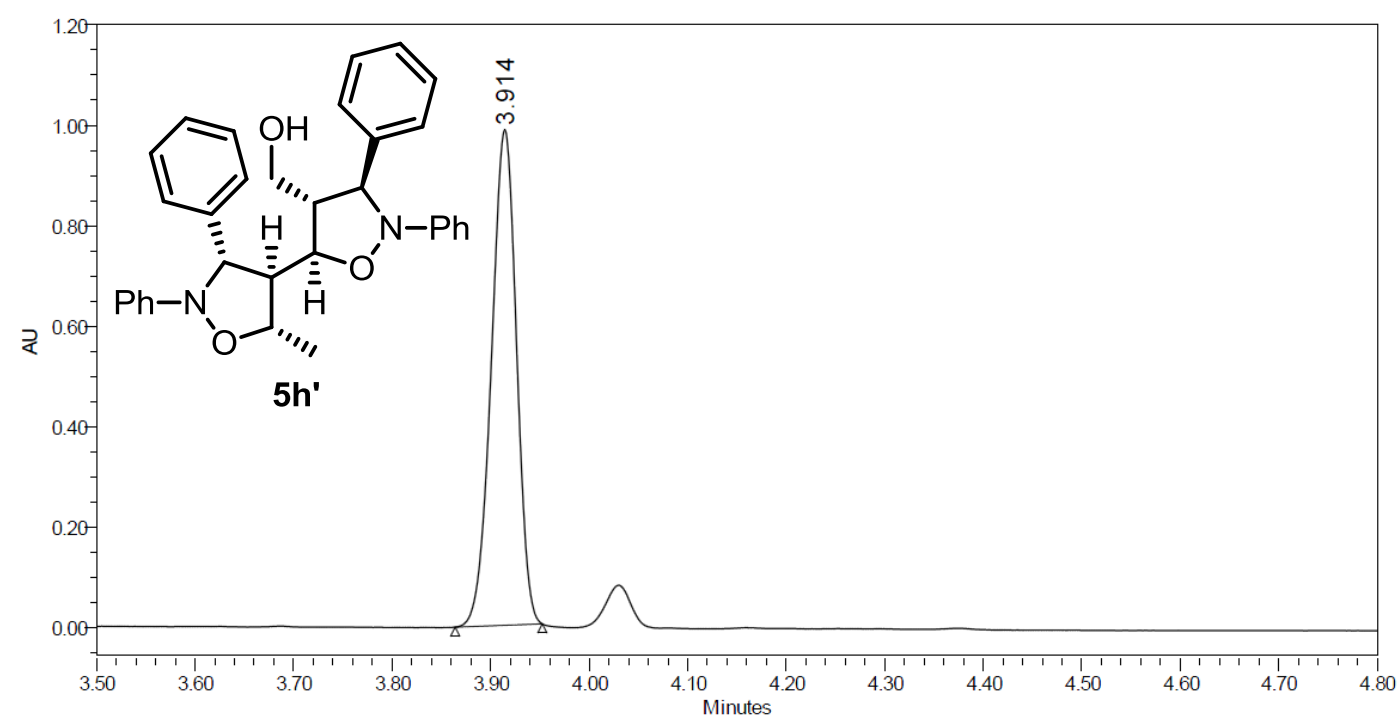

\begin{tabular}{|l|r|r|}
\hline & $\begin{array}{c}\text { Retention Time } \\
\text { (min) }\end{array}$ & $\%$ Area \\
\hline 1 & 3.914 & 100.00 \\
\hline
\end{tabular}




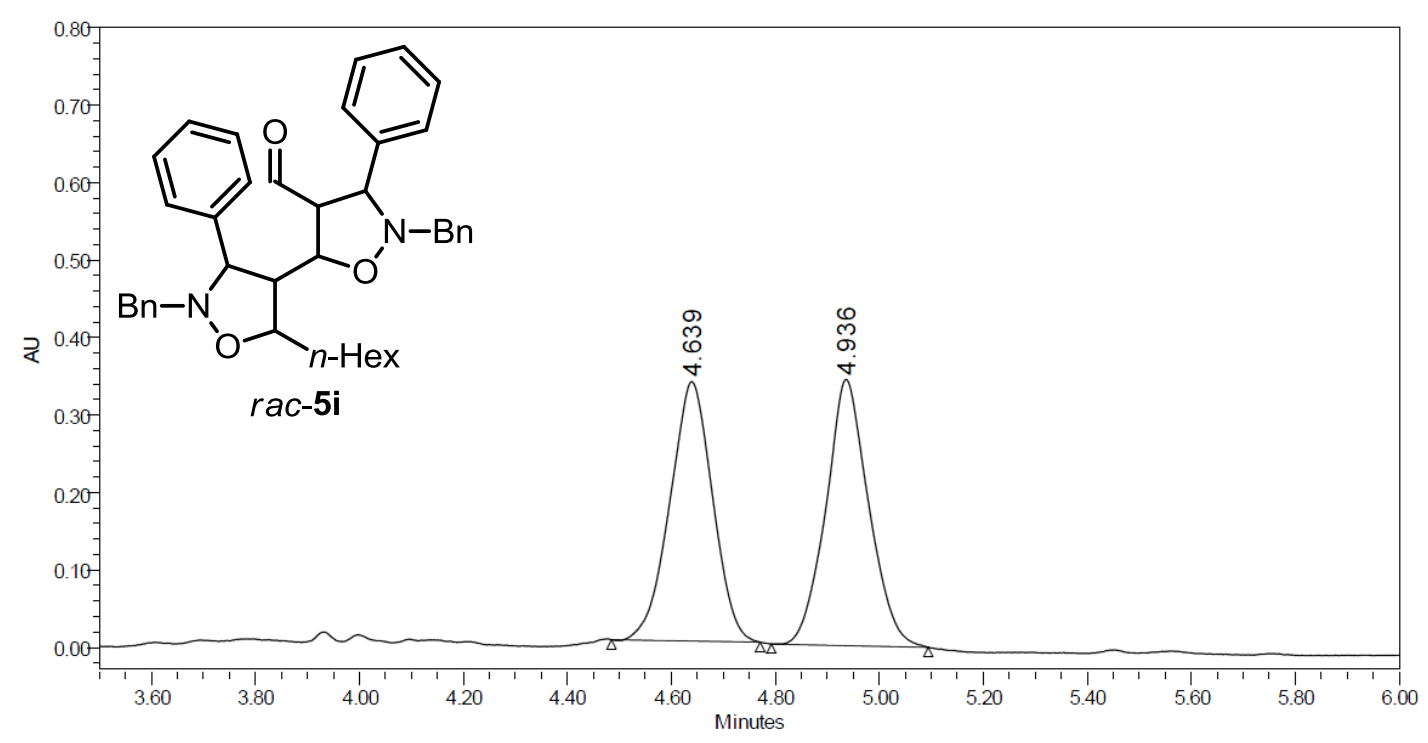

\begin{tabular}{|l|r|r|}
\hline & $\begin{array}{c}\text { Retention Time } \\
\text { (min) }\end{array}$ & \% Area \\
\hline 1 & 4.639 & 49.32 \\
\hline 2 & 4.936 & 50.68 \\
\hline
\end{tabular}

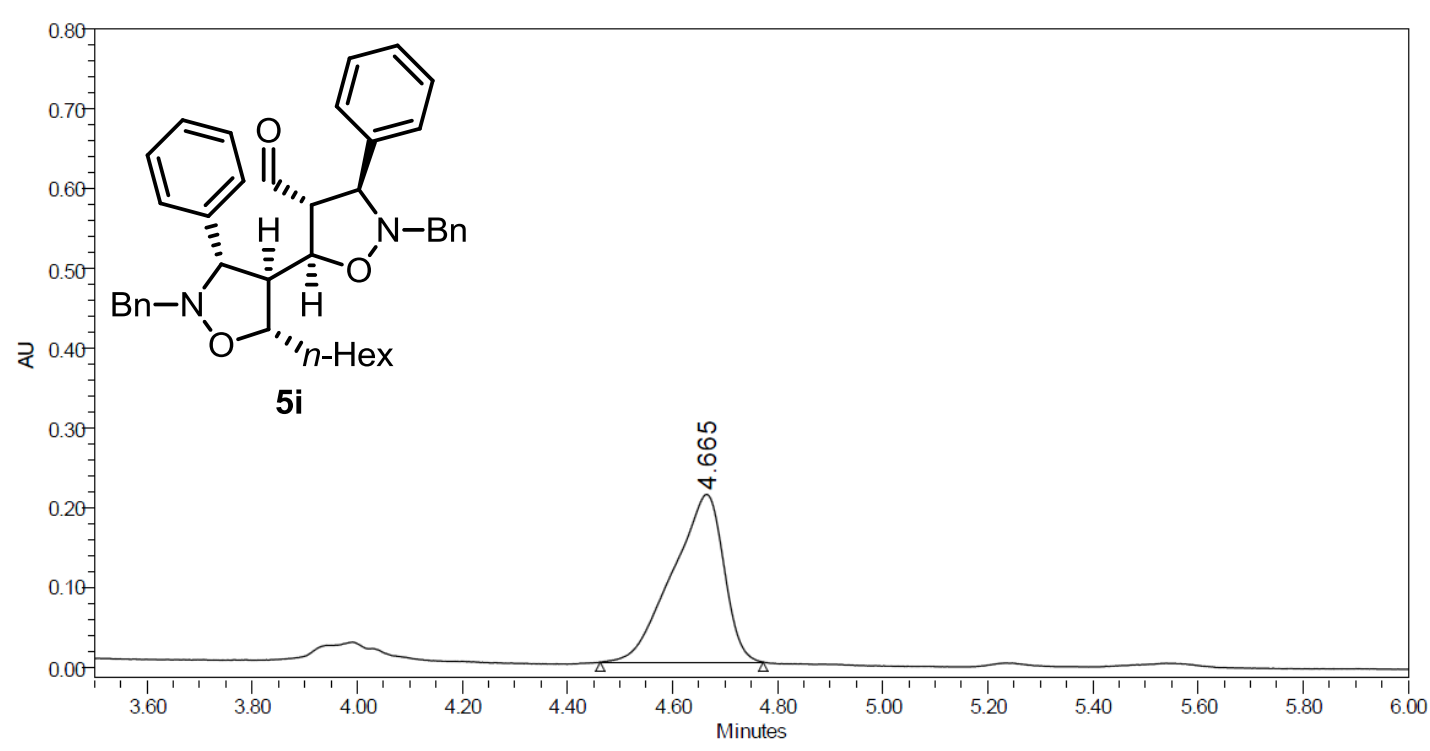

\begin{tabular}{|c|r|r|}
\hline & $\begin{array}{c}\text { Retention Time } \\
(\mathrm{min})\end{array}$ & $\%$ Area \\
\hline 1 & 4.665 & 100.00 \\
\hline
\end{tabular}




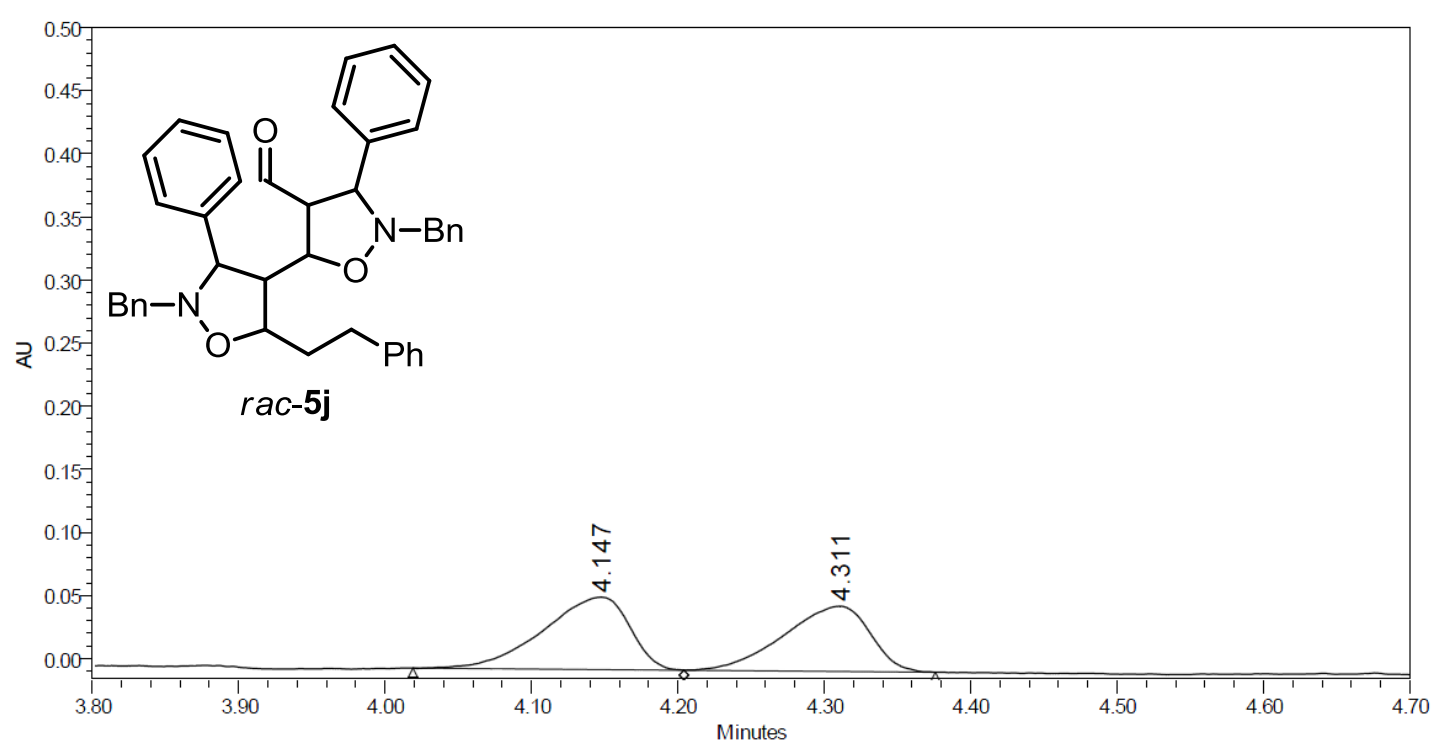

\begin{tabular}{|l|r|r|}
\hline & $\begin{array}{c}\text { Retention Time } \\
\text { (min) }\end{array}$ & \% Area \\
\hline 1 & 4.147 & 52.66 \\
\hline 2 & 4.311 & 47.34 \\
\hline
\end{tabular}

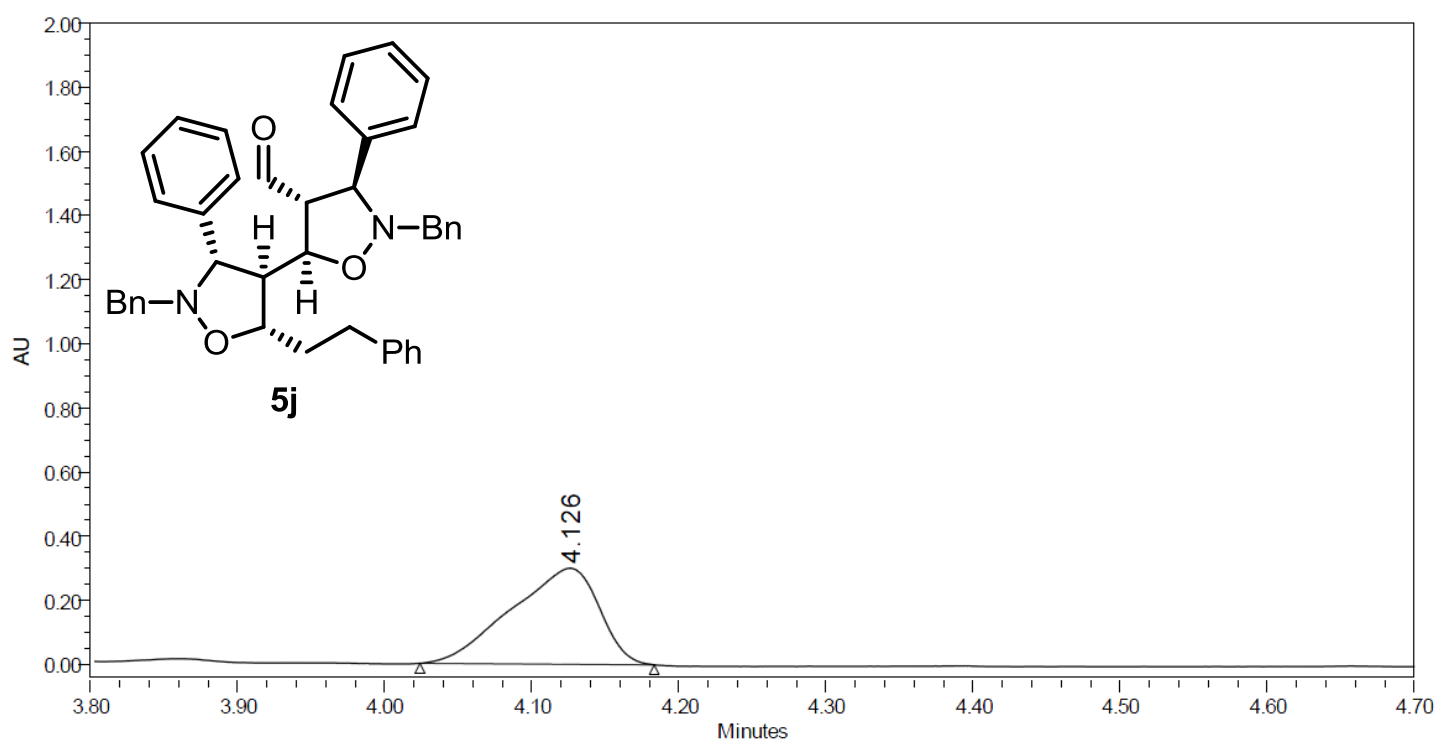

\begin{tabular}{|l|r|r|}
\hline & $\begin{array}{c}\text { Retention Time } \\
(\mathrm{min})\end{array}$ & $\%$ Area \\
\hline 1 & 4.126 & 100.00 \\
\hline
\end{tabular}




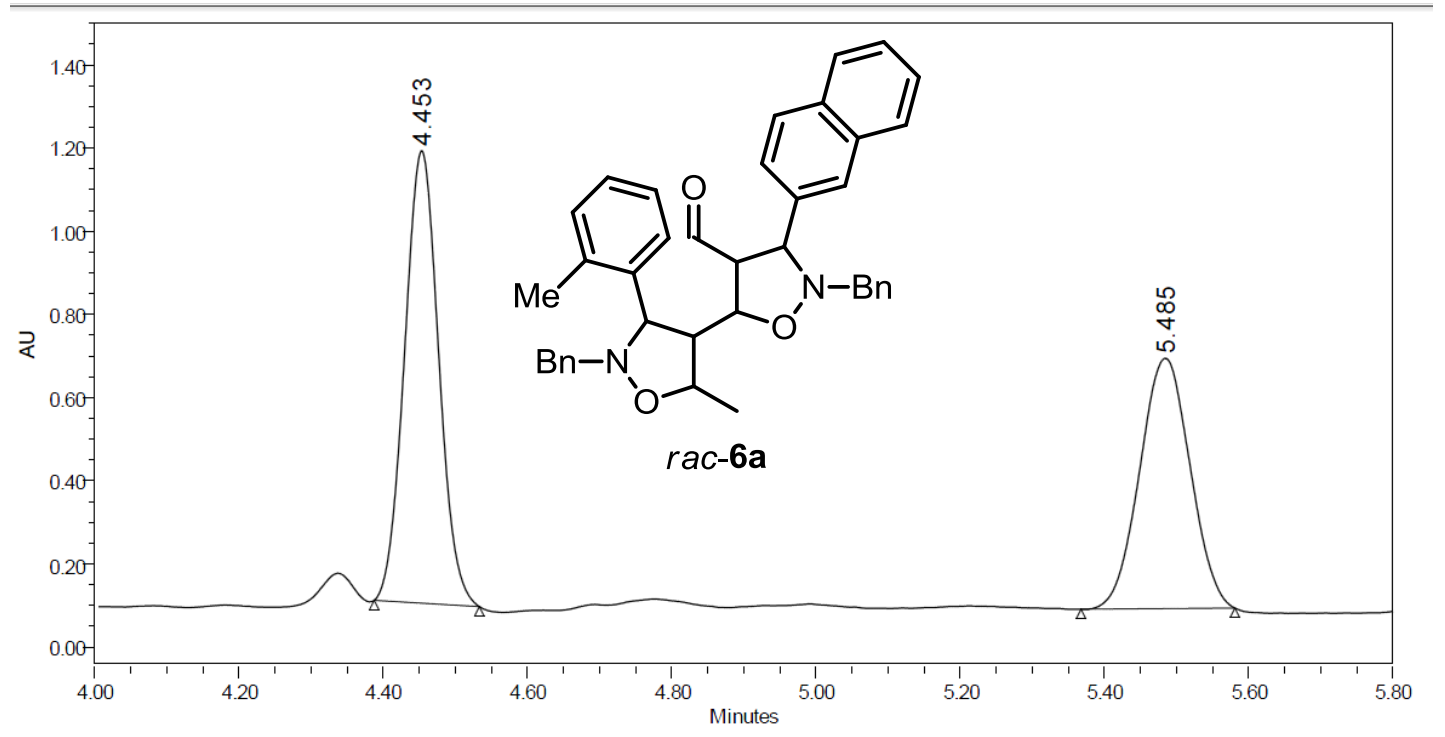

\begin{tabular}{|l|r|r|}
\hline & $\begin{array}{c}\text { Retention Time } \\
(\mathrm{min})\end{array}$ & $\%$ Area \\
\hline 1 & 4.453 & 55.03 \\
\hline 2 & 5.485 & 44.97 \\
\hline
\end{tabular}

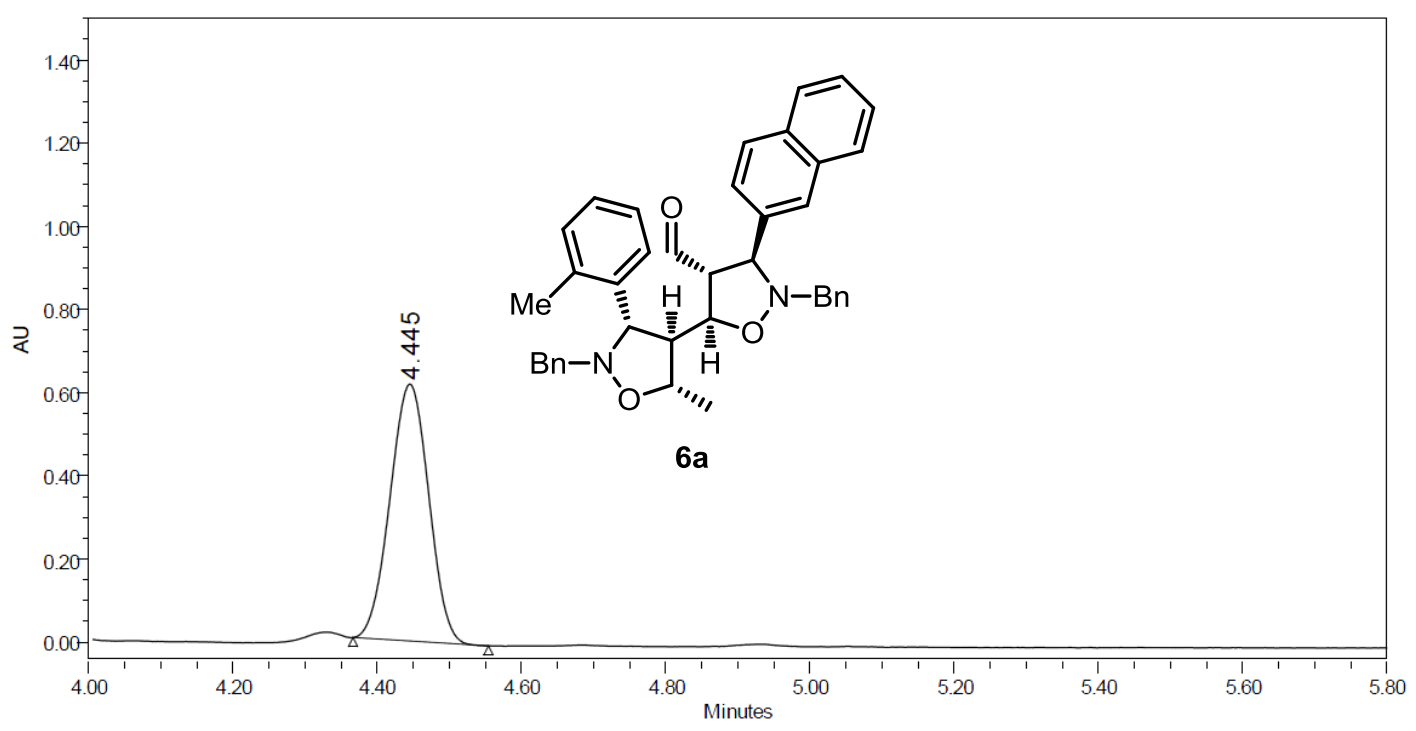

\begin{tabular}{|c|c|c|}
\hline & $\begin{array}{c}\text { Retention Time } \\
\text { (min) }\end{array}$ & $\%$ Area \\
\hline 1 & 4.445 & 100.00 \\
\hline
\end{tabular}




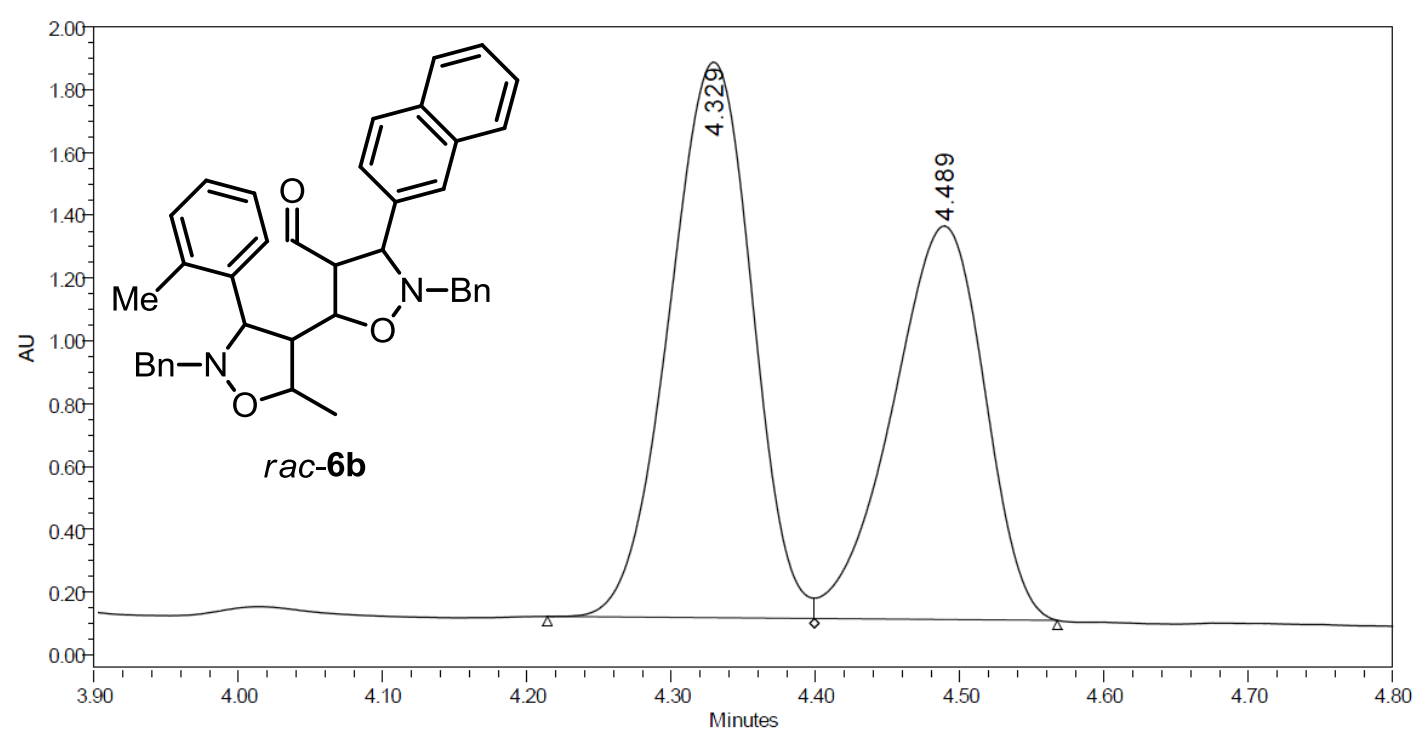

\begin{tabular}{|c|r|r|}
\hline & $\begin{array}{c}\text { Retention Time } \\
\text { (min) }\end{array}$ & \% Area \\
\hline 1 & 4.329 & 55.87 \\
\hline 2 & 4.489 & 44.13 \\
\hline
\end{tabular}

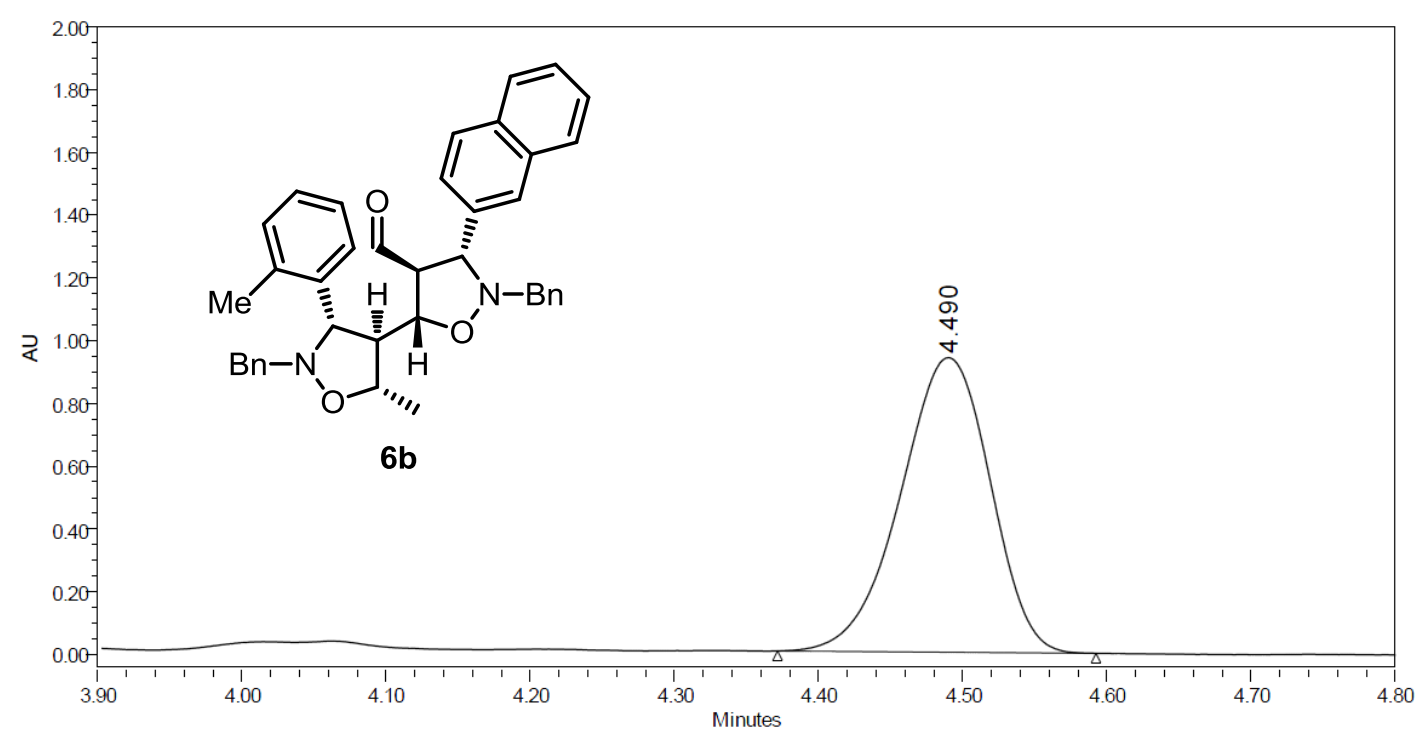

\begin{tabular}{|c|c|c|}
\hline & $\begin{array}{c}\text { Retention Time } \\
(\mathrm{min})\end{array}$ & $\%$ Area \\
\hline 1 & 4.490 & 100.00 \\
\hline
\end{tabular}




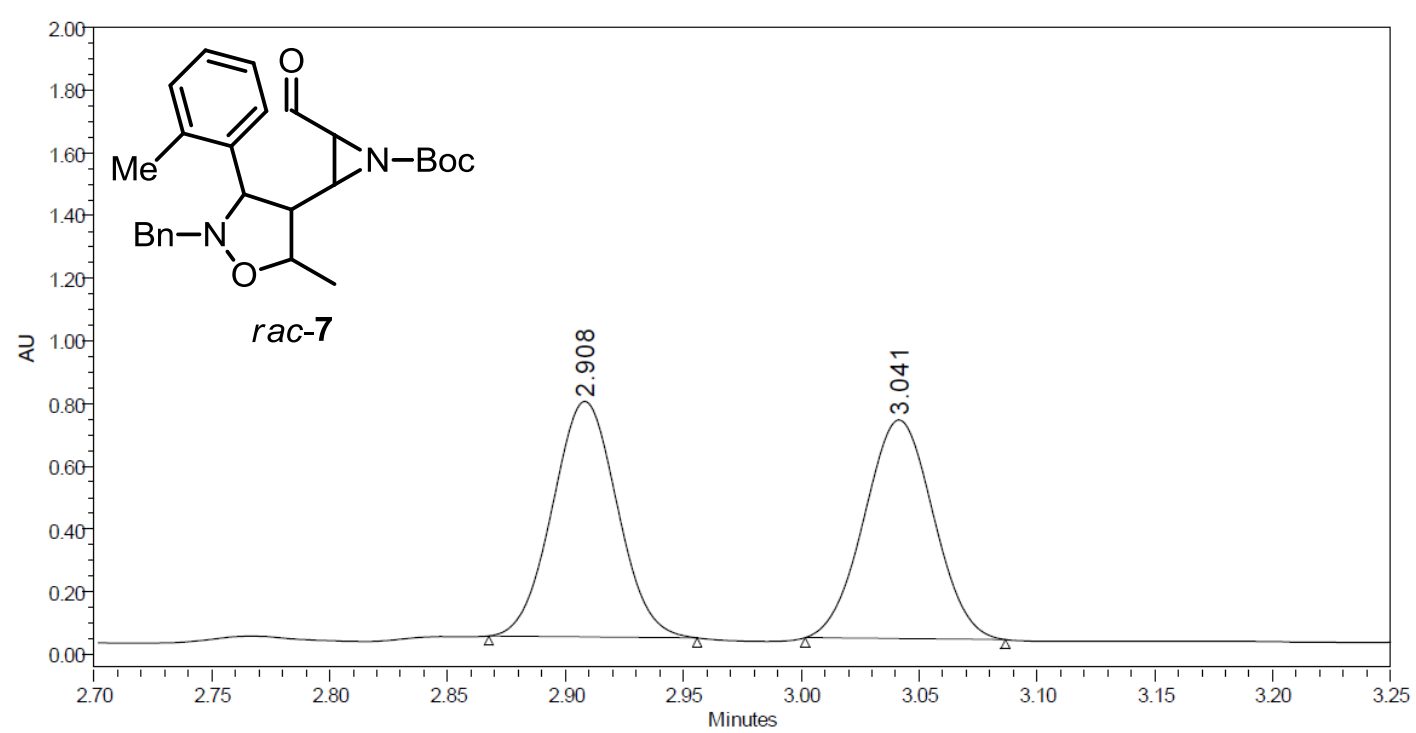

\begin{tabular}{|l|r|r|}
\hline & $\begin{array}{c}\text { Retention Time } \\
(\mathrm{min})\end{array}$ & $\%$ Area \\
\hline 1 & 2.908 & 50.28 \\
\hline 2 & 3.041 & 49.72 \\
\hline
\end{tabular}

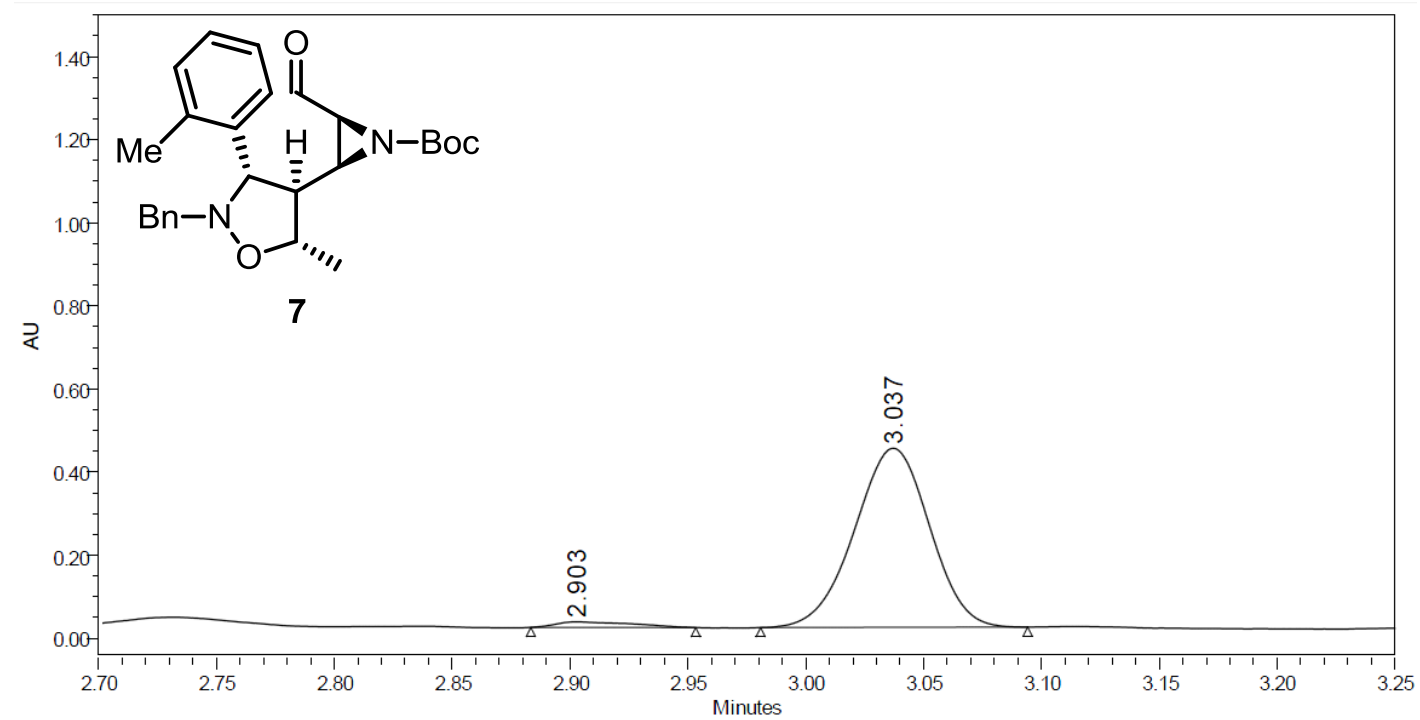

\begin{tabular}{|l|r|r|}
\hline & $\begin{array}{c}\text { Retention Time } \\
\text { (min) }\end{array}$ & \% Area \\
\hline 1 & 2.903 & 3.10 \\
\hline 2 & 3.037 & 96.90 \\
\hline
\end{tabular}




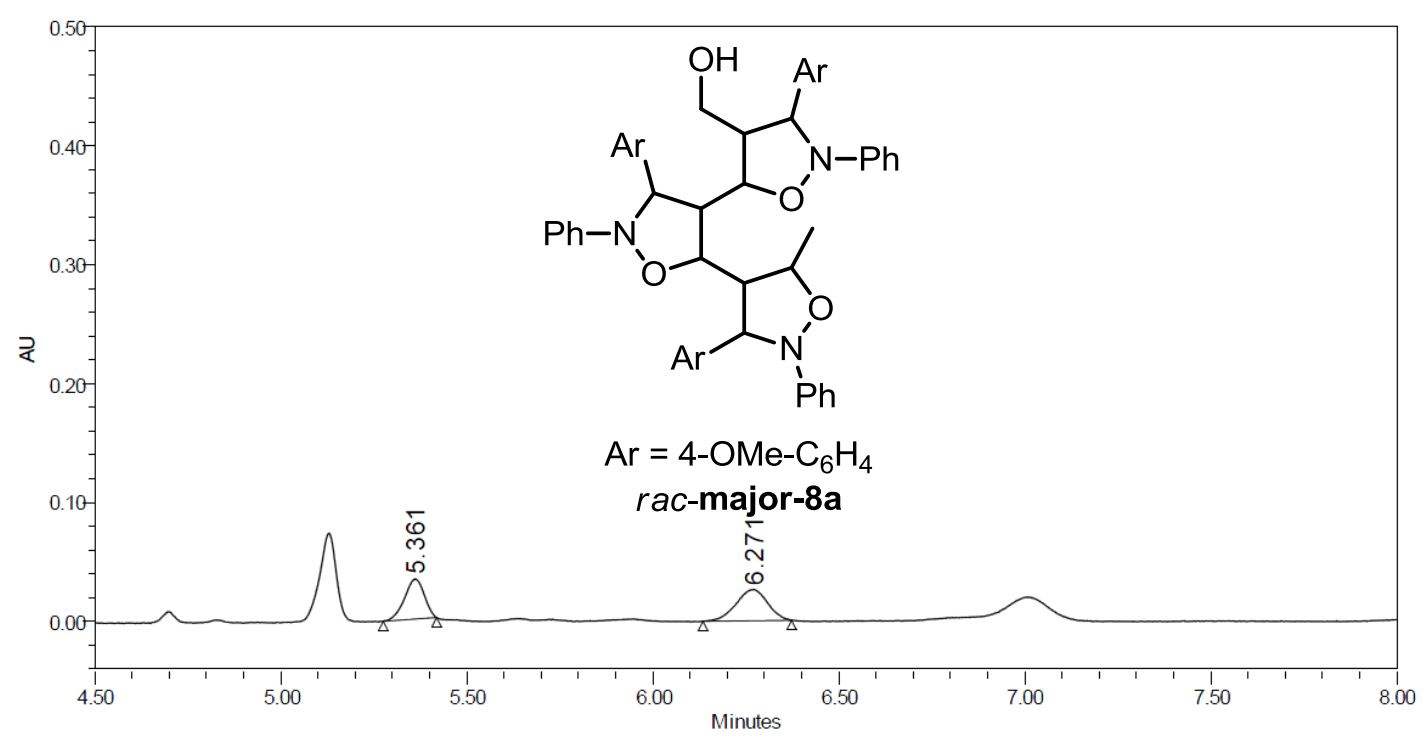

\begin{tabular}{|l|r|r|}
\hline & $\begin{array}{c}\text { Retention Time } \\
\text { (min) }\end{array}$ & $\%$ Area \\
\hline 1 & 5.361 & 44.51 \\
\hline 2 & 6.271 & 55.49 \\
\hline
\end{tabular}

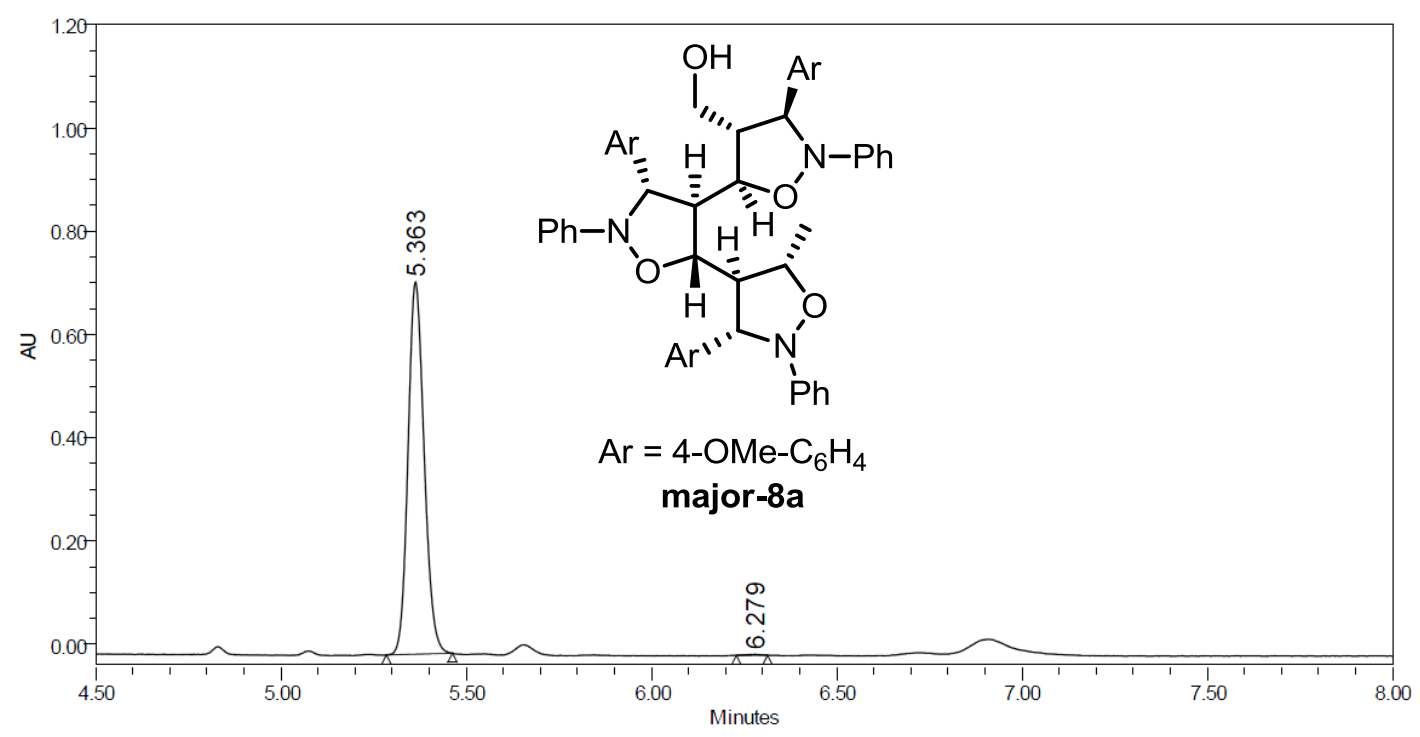

\begin{tabular}{|r|r|r|}
\hline & $\begin{array}{c}\text { Retention Time } \\
(\mathrm{min})\end{array}$ & $\%$ Area \\
\hline 1 & 5.363 & 99.77 \\
\hline 2 & 6.279 & 0.23 \\
\hline
\end{tabular}




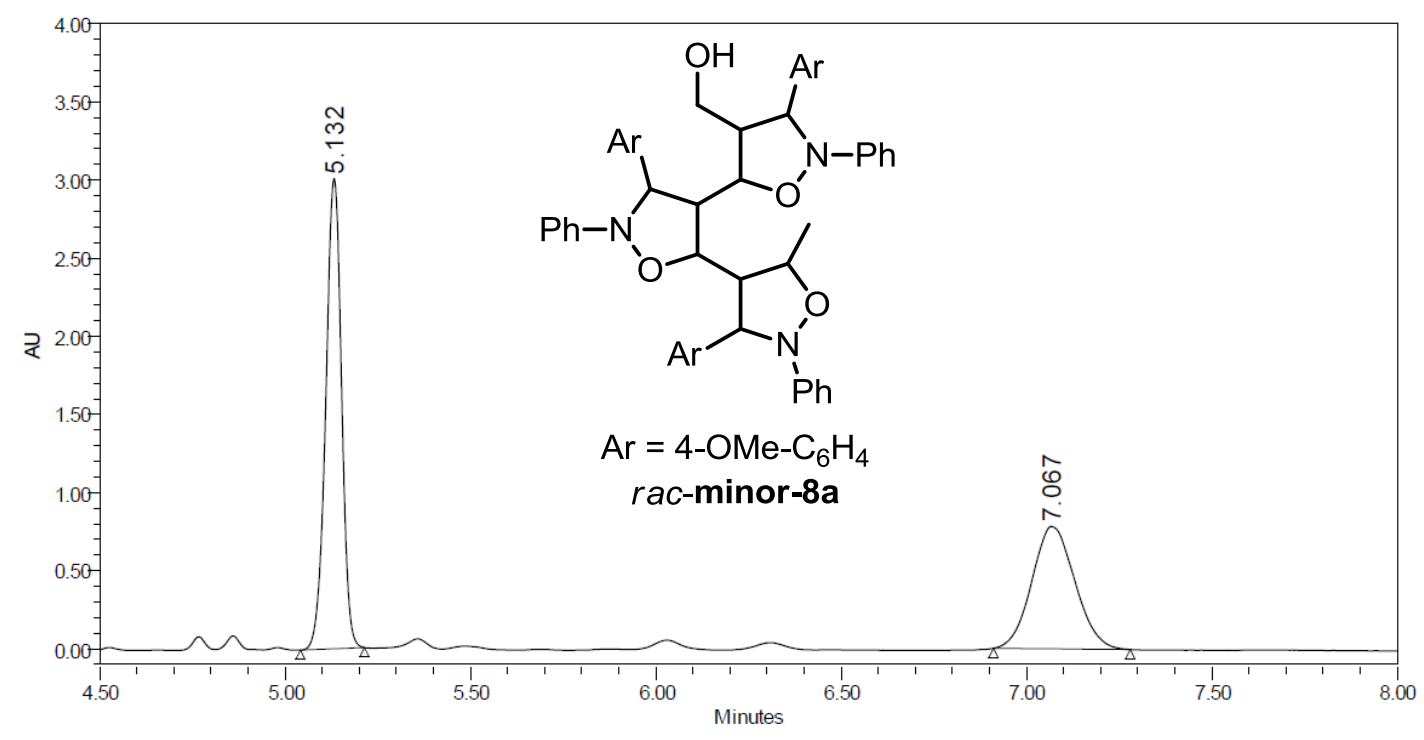

\begin{tabular}{|l|r|r|}
\hline & $\begin{array}{c}\text { Retention Time } \\
\text { (min) }\end{array}$ & $\%$ Area \\
\hline 1 & 5.132 & 58.42 \\
\hline 2 & 7.067 & 41.58 \\
\hline
\end{tabular}

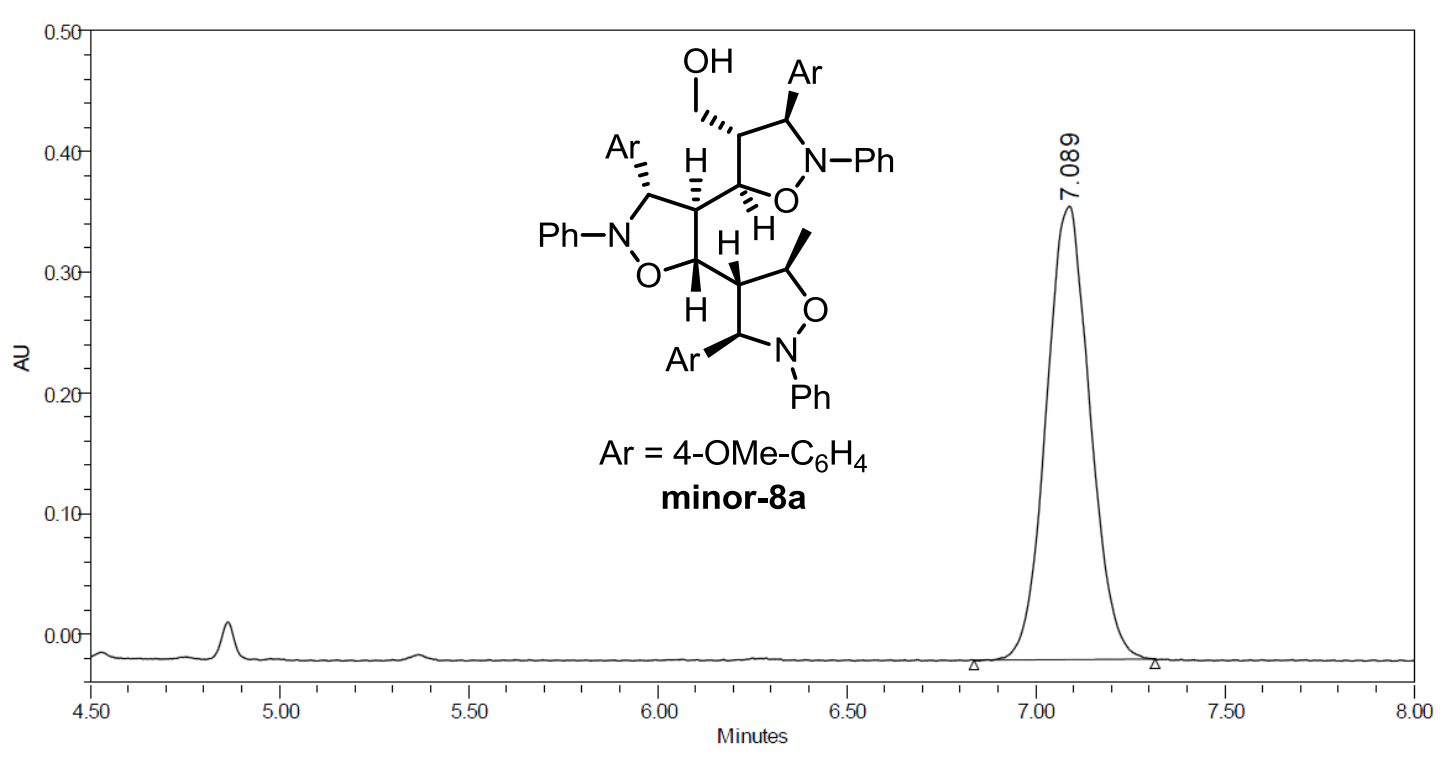

\begin{tabular}{|c|c|c|}
\hline & $\begin{array}{c}\text { Retention Time } \\
\text { (min) }\end{array}$ & $\%$ Area \\
\hline 1 & 7.089 & 100.00 \\
\hline
\end{tabular}




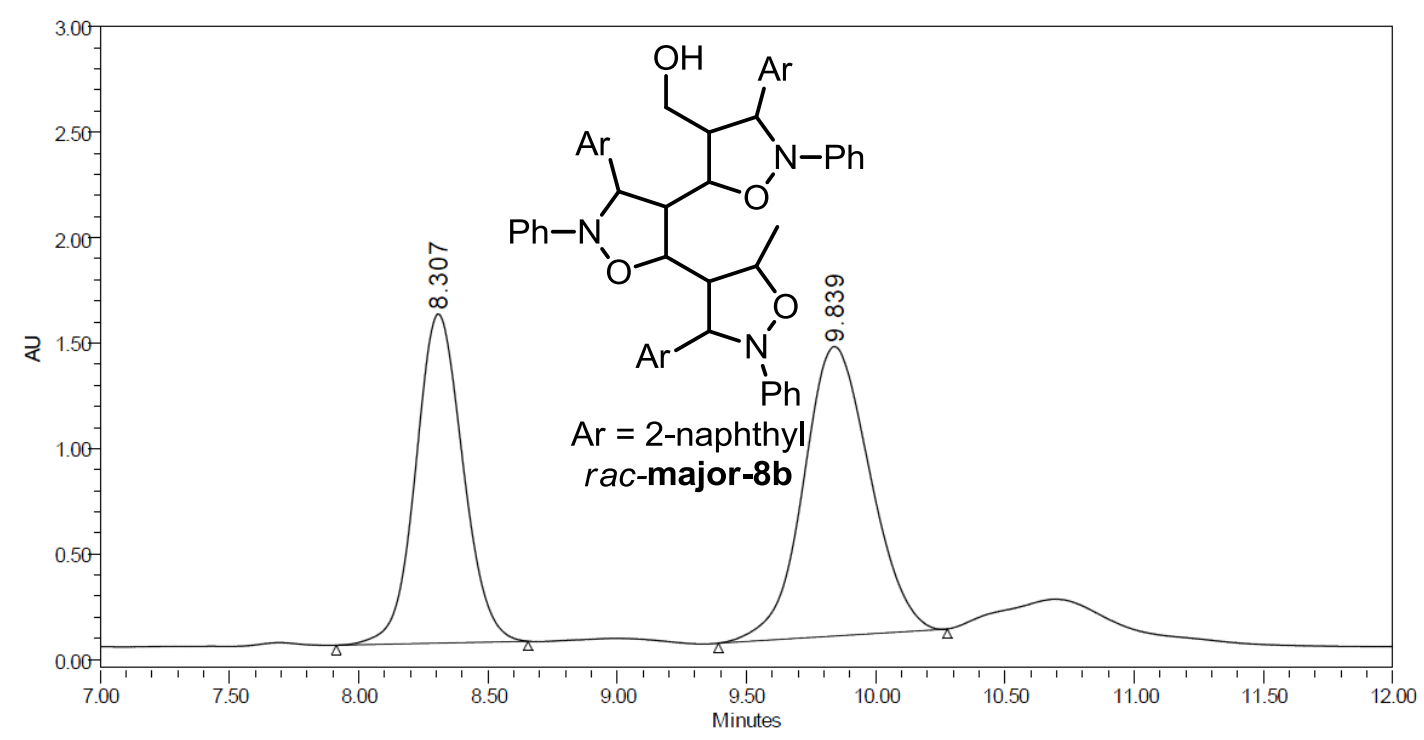

\begin{tabular}{|l|r|r|}
\hline & $\begin{array}{c}\text { Retention Time } \\
\text { (min) }\end{array}$ & $\%$ Area \\
\hline 1 & 8.307 & 44.63 \\
\hline 2 & 9.839 & 55.37 \\
\hline
\end{tabular}

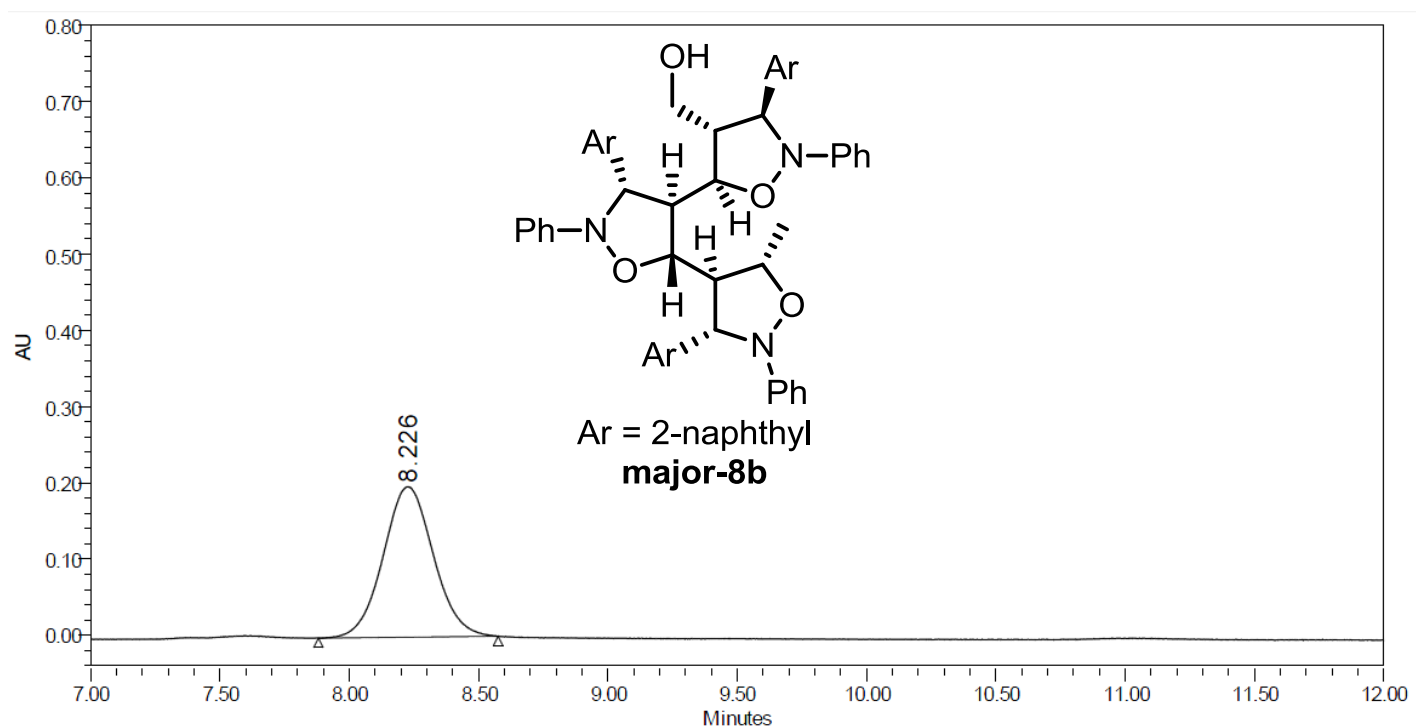

\begin{tabular}{|c|r|r|}
\hline & $\begin{array}{c}\text { Retention Time } \\
\text { (min) }\end{array}$ & $\%$ Area \\
\hline 1 & 8.226 & 100.00 \\
\hline
\end{tabular}




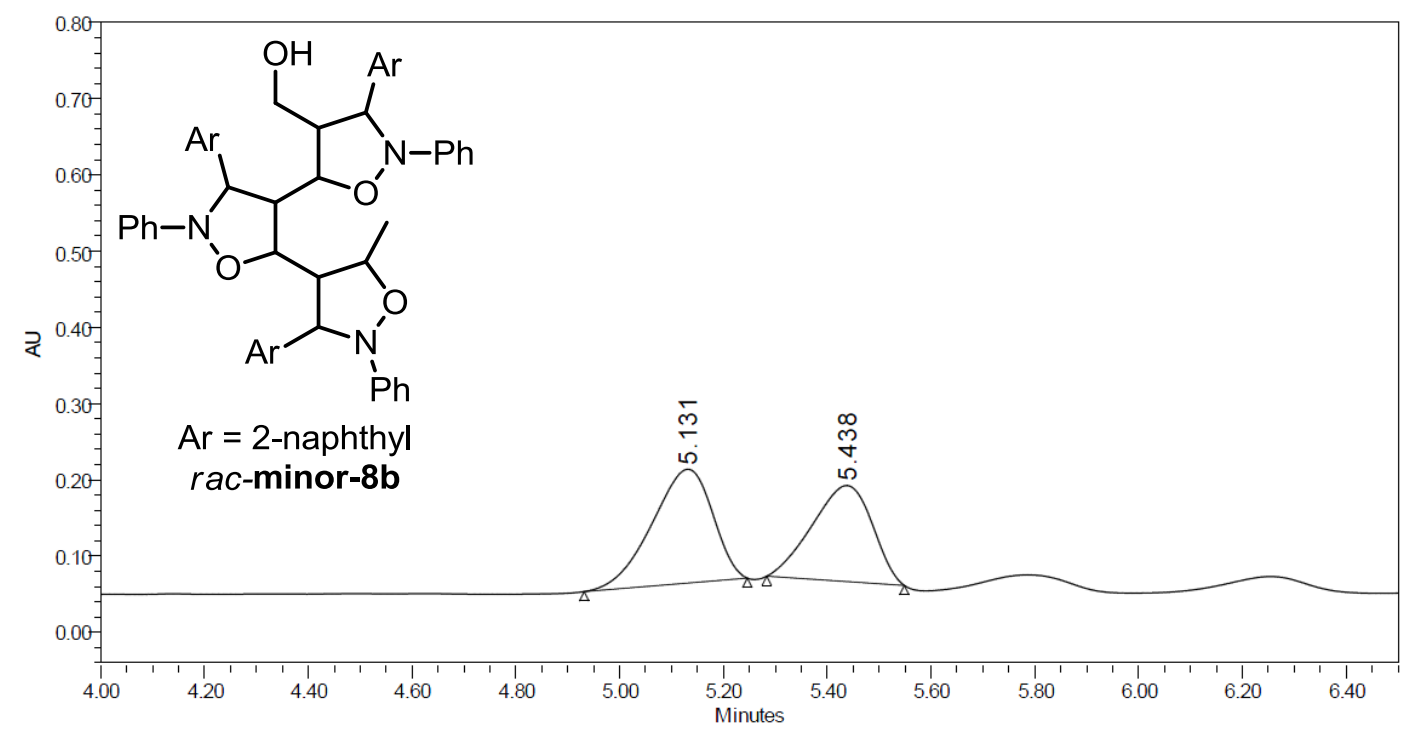

\begin{tabular}{|l|r|r|}
\hline & $\begin{array}{c}\text { Retention Time } \\
(\mathrm{min})\end{array}$ & $\%$ Area \\
\hline 1 & 5.131 & 54.43 \\
\hline 2 & 5.438 & 45.57 \\
\hline
\end{tabular}

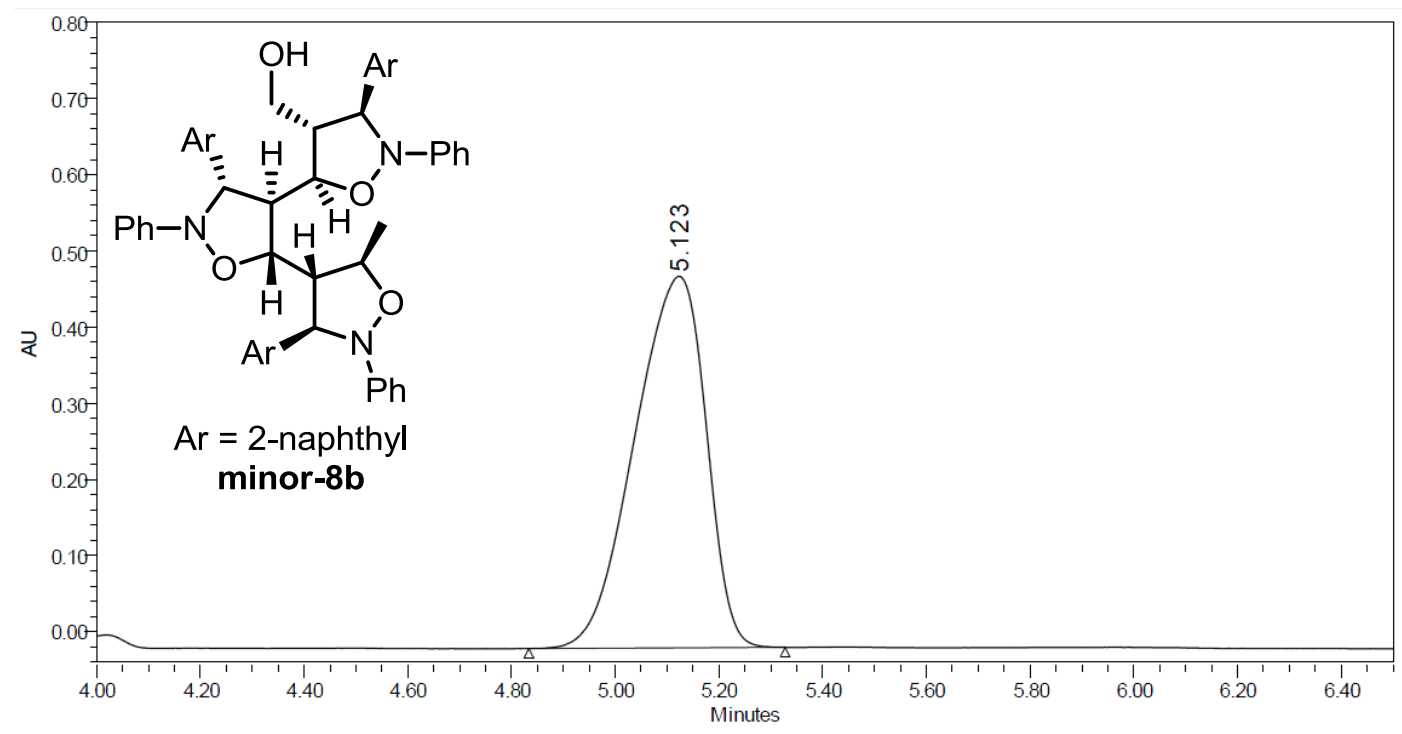

\begin{tabular}{|l|r|r|}
\hline & $\begin{array}{c}\text { Retention Time } \\
(\mathrm{min})\end{array}$ & $\%$ Area \\
\hline 1 & 5.123 & 100.00 \\
\hline
\end{tabular}




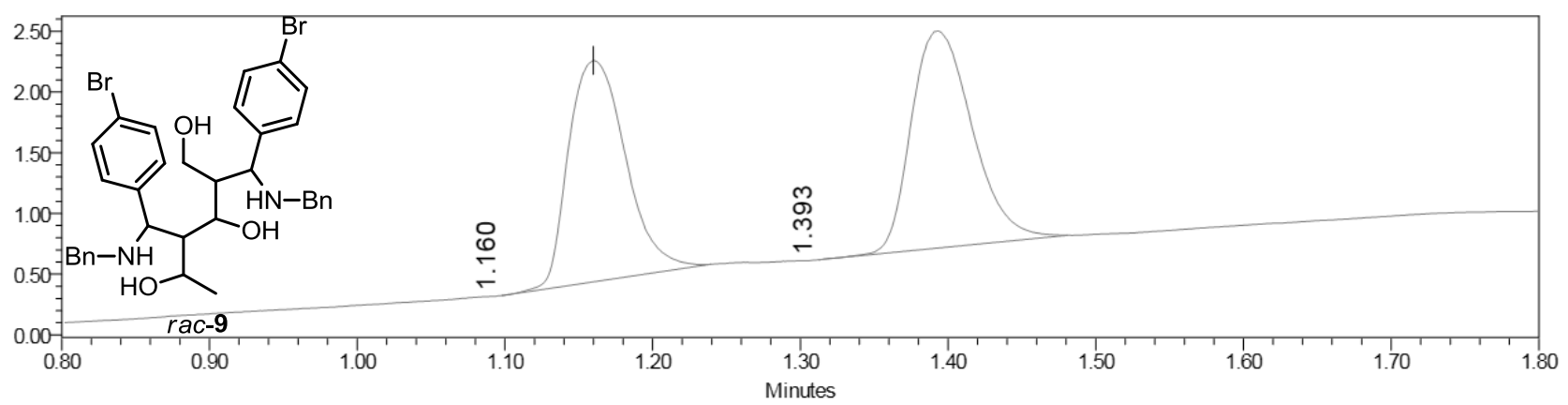

\begin{tabular}{c|c}
$\begin{array}{c}\text { Retention } \\
\text { Time } \\
(\mathrm{min})\end{array}$ & \% Area \\
\hline 1.160 & 48.41 \\
\hline 1.393 & 51.59
\end{tabular}

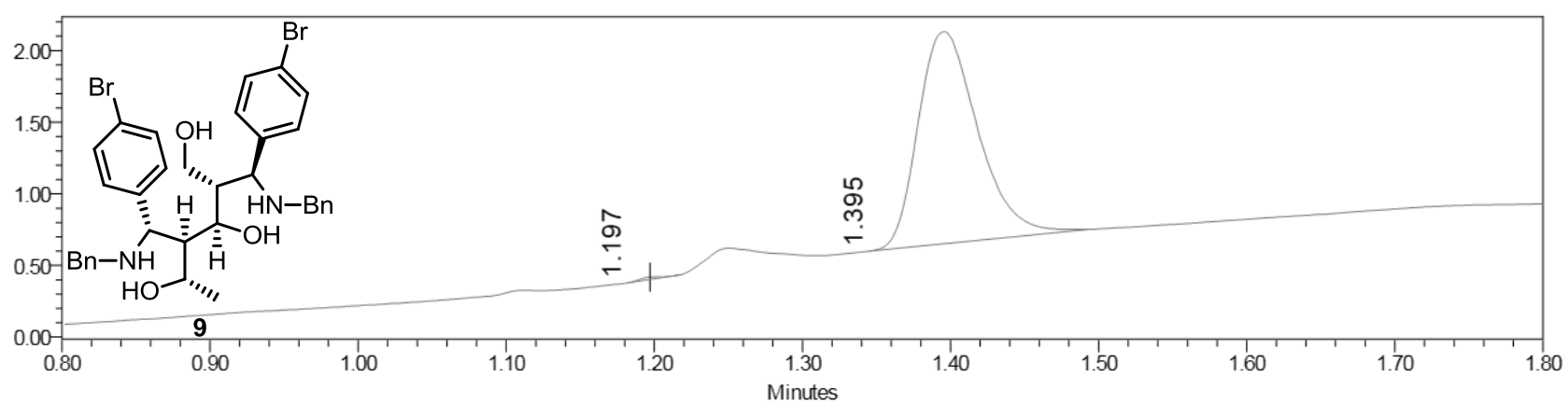

\begin{tabular}{c|c}
$\begin{array}{c}\text { Retention } \\
\text { Time } \\
\text { (min) }\end{array}$ & $\%$ Area \\
\hline 1.197 & 0.36 \\
\hline 1.395 & 99.64
\end{tabular}

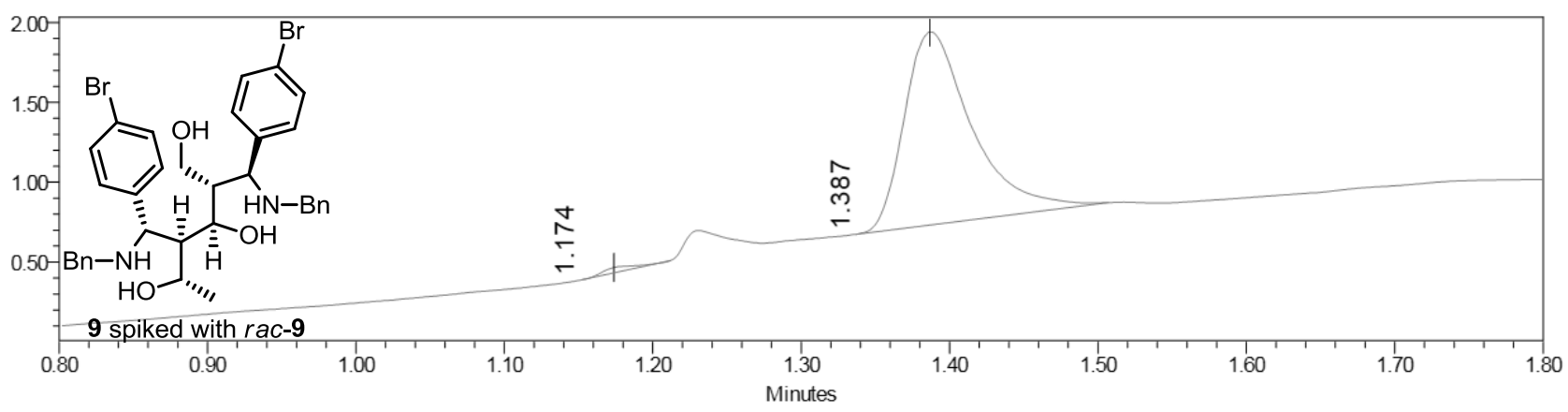

\begin{tabular}{c|c}
$\begin{array}{c}\text { Retention } \\
\text { Time } \\
(\mathrm{min})\end{array}$ & $\%$ Area \\
\hline 1.174 & 1.24 \\
\hline 1.387 & 98.76
\end{tabular}

